Aus dem Institut für Tierzucht und Haustiergenetik der Georg-August-Universität Göttingen

\title{
Ableitung der Wirtschaftlichkeitskoeffizienten und optimalen Indexgewichte des Gesamtzuchtwertes für die deutschen Milch- und Zweinutzungsrassen unter Berücksichtigung aktueller und erwarteter zukünftiger Rahmenbedingungen
}

\author{
Dissertation \\ zur Erlangung des Doktorgrades \\ der Fakultät für Agrarwissenschaften \\ der Georg-August-Universität Göttingen
}

vorgelegt von

Bianca Lind

geboren in Kassel 
D7

1. Referent:

2. Korreferentin:

Tag der mündlichen Prüfung:
Prof. Dr. Henner Simianer

PD Dr. Roswitha Baumung

19. Juli 2007 
Der Abschied von einer langen und wichtigen Arbeit ist immer mehr traurig als erfreulich.

Friedrich Schiller 


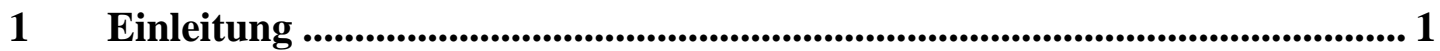

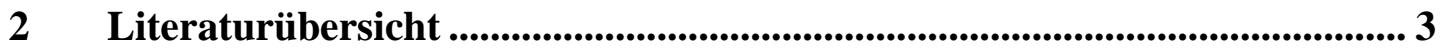

2.1 Ableitung wirtschaftlicher Gewichte .................................................. 3

2.2 Übersicht über die Methoden ................................................................. 5

2.2.1 Nicht-objektive Methoden ……………................................. 6

2.2.2 Objektive Methoden.............................................................. 7

2.3 Ergebnisse zur Schätzung wirtschaftlicher Gewichte ........................... 10

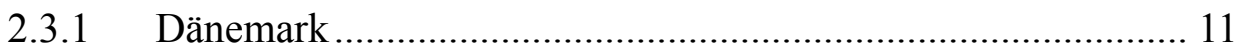

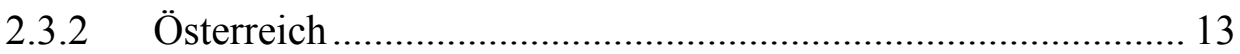

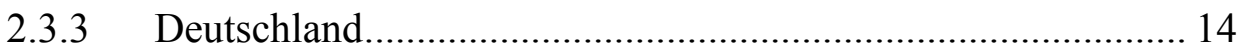

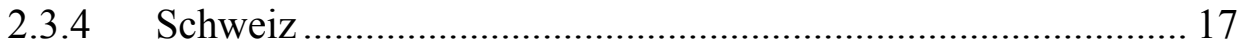

2.3.5 Weitere Arbeiten zu wirtschaftlichen Gewichten..................... 20

2.4 Indexselektion und Gesamtzuchtwert .............................................. 24

2.5 Wirtschaftliche Gewichte in der Zuchtwertschätzung .......................... 26

2.5.1 Gesamtzuchtwert Holstein ..................................................... 29

2.5.2 Gesamtzuchtwert Fleckvieh und Braunvieh .......................... 32

3 Berechnung der wirtschaftlichen Gewichte .................................................. 34

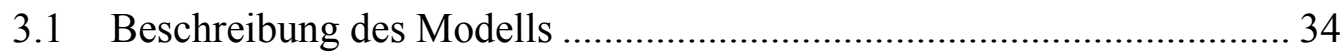

3.1.1 Mittelwerte und Varianzen der wirtschaftlichen Gewichte ....................................................................... 35

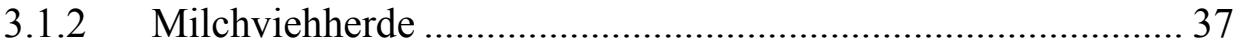

3.1.2.1 Kalbezyklus und Nutzungsdauer .............................. 37

3.1.2.2 Milchleistung und Körpergewicht ............................ 37

3.1.2.3 Grundfutteraufnahme und Bedarf in der

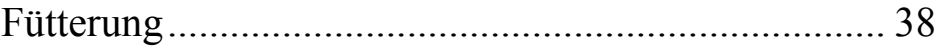

3.1.2.4 Durchschnittliche Klassenergebnisse beim

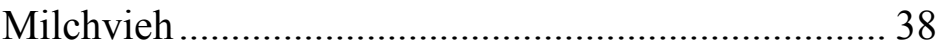

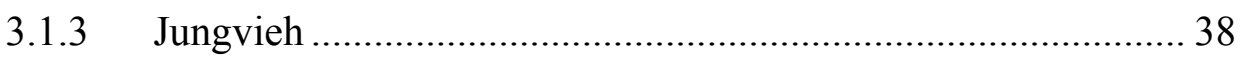

3.1.3.1 Herdenaufbau und Fütterung ................................... 39

3.1.3.2 Ergebnisse der Jungviehaufzucht............................. 39

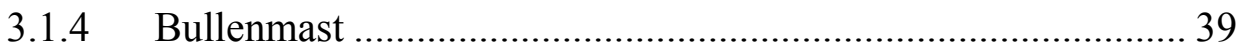

3.1.4.1 Herdenaufbau und Fütterung ................................... 40

3.1.4.2 Ergebnisse der Bullenmast......................................... 40

3.1.5 Gesamtergebnisse............................................................... 40 


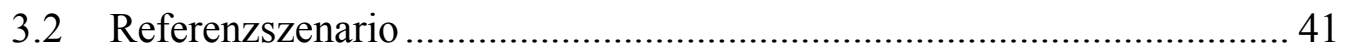

3.2.1 Aufbau und Altersstruktur.................................................. 41

3.2.2 Milchleistung und Milcherlös ............................................... 42

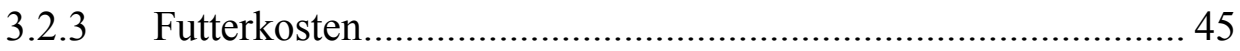

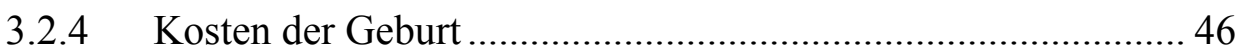

3.2.5 Fruchtbarkeitskennzahlen .................................................. 47

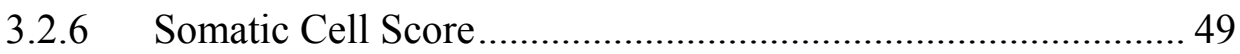

3.2.7 Kosten und Erlöse beim Milchvieh........................................... 50

3.2.8 Kosten und Erlöse in der Bullenmast......................................... 52

3.2.9 Kosten und Erlöse der Kälberaufzucht ..................................... 54

3.3 Ableitung der wirtschaftlichen Gewichte.............................................. 56

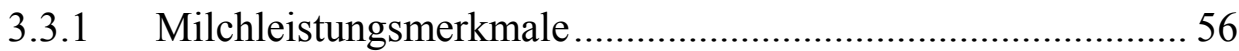

3.3.2 Fleischleistungsmerkmale _..................................................... 57

3.3.3 Funktionale Merkmale ............................................................... 58

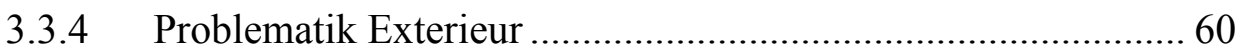

3.4 Ableitung optimaler Indexgewichte (b-Werte) ..................................... 63

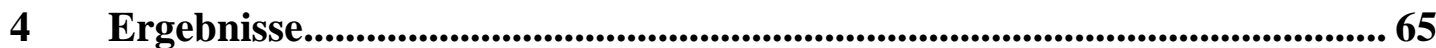

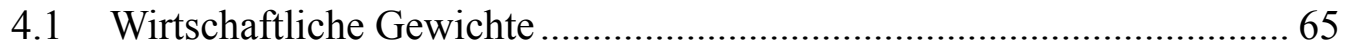

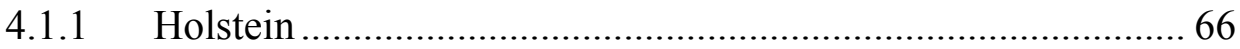

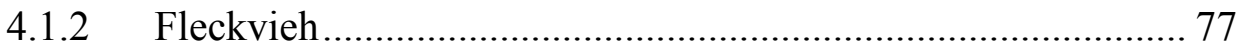

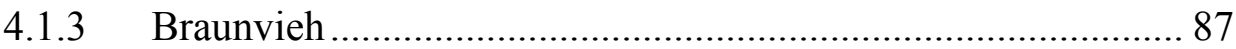

4.1.4 Vergleich der drei Rassen.................................................... 93

4.2 Indexgewichte am Beispiel Holstein...................................................... 95

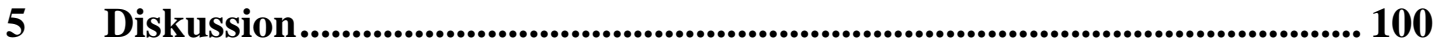

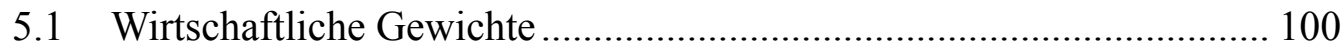

5.1.1 Beurteilung des Modells ...................................................... 100

5.1.2 Einschätzung der Szenarien ................................................. 103

5.1.3 Milchquotenregelungen ....................................................... 105

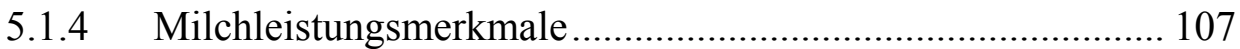

5.1.5 Funktionale Merkmale .......................................................... 109

5.1.6 Fleischleistungsmerkmale .................................................... 116

5.1.7 Weitere Merkmale ............................................................. 118

5.1.8 Zusammensetzung des Gesamtzuchtwertes .......................... 118

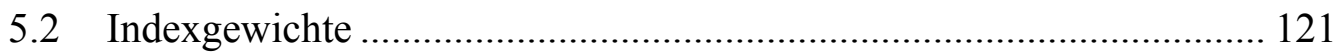




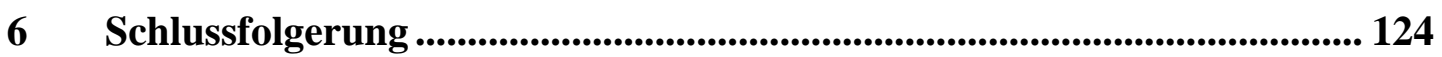

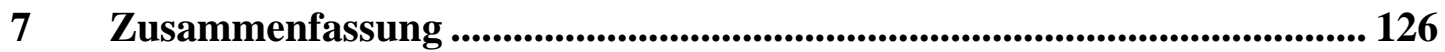

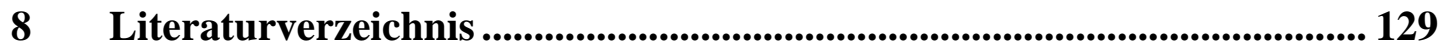




\section{Tabellenverzeichnis}

Tab. 2.1

Tab. 2.2

Tab. 2.3

Tab. 2.4

Tab. 2.6

Tab. 2.7

Tab. 2.8
Grenznutzen in $€$ je Merkmalseinheit pro Kuh und Jahr für Produktions- und funktionale Merkmale in einem Szenario bei einer Merkmalsverbesserung von jeweils 20\% in allen Merkmalen und in einem Szenario mit aktuellen Produktionsbedingungen (nach Nielsen, 2004)

Wirtschaftliche Gewichte für Produktions- und funktionale Merkmale in $€$ je genetischer Standardabweichung bei der Rasse Fleckvieh (nach Miesenberger, 1997)

Grenznutzen der Leistungs- und funktionalen Merkmale für Schwarzbunte unter alternativen wirtschaftlichen Rahmenbedingungen in $€$ je Merkmalseinheit (nach Mack, 1996)

Grenznutzen der Leistungs- und funktionalen Merkmale für Schwarzbunte in $€$ je Merkmalseinheit unter verschiedenen Szenarien (nach Weidele, 1996)

Berechnung der wirtschaftlichen Gewichte in $€$ je genetischer Standardabweichung in der Schweiz (nach Böbner, 1994) .

Übersicht über die wirtschaftlichen Gewichte bei Holstein und Fleckvieh in der Schweiz ohne Milchquotenbeschränkung (Baumung und Sölkner, 1998) ....

Relative wirtschaftliche Gewichte (\%) der Holsteinpopulation in Estland unter Milchquotenbeschränkungen (Pärna et al., 2003).

Relative Gewichtung der Merkmalsgruppen Milch, Fleisch, Fitness und Melkbarkeit (MBK) im Gesamtzuchtwert „Fleckvieh 2000“ und „Fleckvieh 2002“ (nach Gredler, 2004) .... 27 
Tab. 2.9

Relative Gewichtung der Merkmalsgruppen Milch, Fleisch, Fitness und Melkbarkeit (MBK) im Gesamtzuchtwert bei „Braunvieh 2002“ und „Braunvieh 2004“ (nach Gredler, 2004)

Tab. 3.1

Tab. 3.2

Tab. 3.3

Tab. 3.4

Tab. 3.5

Tab. 3.6

Tab. 3.7

Tab. 3.8

Tab. 3.9
Aufteilung der lebenden Kühe auf die einzelnen Laktationen bei den Rassen Fleckvieh und Braunvieh (LfL, 2004a) sowie Holstein (LKV, 2004)

305-Tage-Leistung für Milchmenge (kg), Fett- (\%) und Eiweißgehalt (\%) für die Rassen Fleckvieh und Braunvieh (LKV, 2005) sowie Holstein (LKV, 2004)....

Alterungsfaktoren für die Berechnung des Milchleistungspotentials in den einzelnen Laktationen bei Fleckvieh und Braunvieh (LKV, 2005) sowie Holstein (LKV, 2004)

Milchauszahlungspreis für die Komponenten Grundpreis sowie Fett- und Eiweißpreis in $€$ je Einheit für Fleckvieh, Holstein und Braunvieh im Referenzszenario (OECD/FAO, 2005; Seufferlein, 2005)

Angaben $\mathrm{zu}$ den unterstellten Futtermitteln je $\mathrm{kg}$ Trockensubstanz (Jeroch et al., 1999) und deren aktuelle Preise (Landwirtschaftskammer, 2004) 46

Klassifizierung des Kalbeverlaufs (nach VIT, 2006) 46

Verteilung des Kalbeverlaufs auf die einzelnen Klassen in Prozent und ergänzende Angaben zur Berechnung der durchschnittlichen Kosten je Geburt bei Fleckvieh und Braunvieh (ZuchtData, 2005a) sowie Holstein (Wünsch, 1999)

Biologische Parameter im Betrieb bei den Rassen Fleckvieh und Braunvieh (LfL, 2004a) sowie Holstein (Landwirtschaftskammer, 2004) .................................................. 48

Konzeptionsraten für Fleckvieh (Fürst, 2006) 48 
Tab. $3.10 \quad$ Konzeptionsraten für Holstein (Reinhardt, 2006)

Tab. $3.11 \quad$ Konzeptionsrate für Braunvieh (Fürst, 2006)

Tab. 3.12 Aufteilung der Rassen Fleckvieh, Holstein und Braunvieh auf die Klassen des Somatic Cell Score (LfL, 2004b; ZuchtData, 2005a)

Tab. 3.13 Arbeitszeit und Arbeitskosten im Betrieb bei den Rassen Fleckvieh, Holstein und Braunvieh (KTBL, 2005)

Tab. 3.14 Kosten im Betrieb bei den Rassen Fleckvieh, Holstein und Braunvieh (KTBL, 2005)

Tab. 3.15

Verkaufs- und Schlachtdaten der Färsen und Kühe bei den Rassen Fleckvieh und Braunvieh (LfL, 2005) sowie Holstein (Weiss, 2005)

Tab. 3.16 Parameter der Bullenmast bei den Rassen Fleckvieh und Braunvieh (LKV, 2005)

Tab. 3.17 Jungbullenpreise und Anteile in den einzelnen Handelsklassen (EUROP) bei Fleckvieh und Braunvieh in Bayern und Baden-Württemberg (BAFF, 2006).

Tab. 3.18

Parameter der Kälberaufzucht bei den Rassen Fleckvieh, Holstein und Braunvieh (KTBL, 2005; Weiss, 2005)....

Tab. 3.19: Genetische Korrelationsmatrix $\left(\mathrm{r}_{\mathrm{g}}\right)$ für die einzelnen Teilzuchtwerte (VIT, 2006) 63

Tab. 3.20 Sicherheiten der Teilzuchtwerte bei einem Testbullen nach Testeinsatz und bei einem geprüften Vererber (Reinhardt, 2006).

Tab. 4.1 Genetische Standardabweichung der einzelnen Merkmale bei Holstein (Reinhardt, 2006) 66

Tab. 4.2

Varianten der Gewichtung des Gesamtzuchtwertes bei Veränderung des relativen wirtschaftlichen Gewichtes (\%) für die Exterieurmerkmale bei Holstein (VIT, 2006) 
Tab. 4.3 Genetische Standardabweichung der einzelnen Merkmale bei Fleckvieh (Dodenhoff, 2005)

Tab. 4.4 Genetische Standardabweichung der einzelnen Merkmale bei Braunvieh (Dodenhoff, 2006) 88

Tab. 4.5 Gewichtung der Teilzuchtwerte im Gesamtzuchtwert nach DHV und eigenen Berechnungen 95

Tab. 4.6 Relative Gewichtung, Sicherheiten und Indexgewichte $\left(b^{*} w\right)$ der Teilzuchtwerte eines Bullen aus der Top-Liste nach der derzeitigen Zuchtwertschätzung (DHV) 96

Tab. 4.7 Relative Gewichtung, Sicherheiten und Indexgewichte $\left(b^{*} w\right)$ der Teilzuchtwerte eines Bullen aus der Top-Liste nach den eigenen Berechnungen

Tab. 4.8 Indexgewichte $\left(b^{*} w\right)$ und Sicherheiten der Teilzuchtwerte im Vergleich bei einem Testbullen nach Testeinsatz und einem geprüften Vererber

Tab. 4.9

Verhältnis der b-Werte in den einzelnen Teilzuchtwerten der Top-10-Bullen zueinander (\%) 98 


\section{Abbildungsverzeichnis}

Abb. 2.1

Abb. 3.1

Abb. 3.2

Abb. 4.1

Abb. 4.2

Abb. 4.3

Abb. 4.5

Abb. 4.6
Relative Gewichtung der Merkmalskomplexe Milchleistung und Lebensdauer sowie Gesundheit und Reproduktion in den Gesamtzuchtwerten einzelner Länder im August 2003 (nach Miglior et al., 2005)

Wirtschaftliche Gewichte und Häufigkeitsverteilung am Beispiel Fettmenge bei Fleckvieh im Referenzszenario 36

Veränderung der Bezahlung der Fett- und Eiweißeinheiten in Deutschland von 1979 bis 2002 in Cent je Einheit (Seufferlein, 2005)

Grenznutzen ( \pm Standardabweichung) der einzelnen Merkmale in $€$ je Merkmalseinheit bei Holstein im Referenzszenario ohne Milchquotenbeschränkung 67

Grenznutzen für die Fett- und Eiweißmenge in Abhängigkeit vom Bezahlungssystem bei der Rasse Holstein (in $€$ je kg) 68

Wirtschaftliche Gewichte ( \pm Standardabweichung) der einzelnen Merkmale in $€$ je genetischer Standardabweichung bei Holstein im Referenzszenario ohne Milchquotenbeschränkung.

Wirtschaftliche Gewichte der einzelnen Merkmale in $€$ je genetischer Standardabweichung bei Holstein ohne und mit Milchquotenbeschränkung im Vergleich 70

Relative wirtschaftliche Gewichte der einzelnen Merkmale (\%) bei Holstein

Einfluss der durchschnittlichen Nutzungsdauer auf die wirtschaftlichen Gewichte $\left(€ / \sigma_{\mathrm{a}}\right)$ der Merkmale Fettmenge (Fett), Nutzungsdauer (ND) und Exterieur (Exterieur) bei Holstein. 
Abb. $4.7 \quad$ Vergleich der relativen wirtschaftlichen Gewichte (\%) der eigenen Berechnungen mit der derzeitigen Gewichtung der Teilzuchtwerte nach DHV bei Holstein (VIT, 2006)

Abb. 4.8

Rangierung ausgewählter Bullen nach RZG (Zuchtwertpunkte) bei unterschiedlichen Anteilen des RZE bei Holstein

Abb. 4.9

Mittelwerte der Relativzuchtwerte der Top-100-Bullen in den drei RZG-Varianten (DHV, neu mit 9\% und neu mit 1\% Gewichtung des RZE).

Abb. 4.10

Grenznutzen ( \pm Standardabweichung) der einzelnen Merkmale in $€$ je Merkmalseinheit bei Fleckvieh im Referenzszenario

Abb. 4.11

Wirtschaftliche Gewichte ( \pm Standardabweichung) der einzelnen Merkmale in $€$ je genetischer Standardabweichung im Referenzszenario bei Fleckvieh.

Abb. 4.12 Grenznutzen ( \pm Standardabweichung) der einzelnen Merkmale in $€$ je Merkmalseinheit bei Fleckvieh im Vergleichsszenario ohne Milchquotenbeschränkungen

Abb. 4.13

Wirtschaftliche Gewichte ( \pm Standardabweichung) der einzelnen Merkmale in $€$ je genetischer Standardabweichung im Vergleichsszenario ohne Milchquotenbeschränkung bei Fleckvieh.

Abb. 4.14 Wirtschaftliche Gewichte der einzelnen Merkmale in $€$ je genetischer Standardabweichung bei Fleckvieh im Vergleich der Szanarien

Abb. 4.15

Anteil der einzelnen Merkmalskomplexe (\%) beim Gesamtzuchtwert (GZW) für Fleckvieh 2006 (Miesenberger, 1999) und den drei vorgestellten Szenarien.

Abb. 4.16 Einfluss des Schlachtpreises für Kühe ( $€$ je kg SG) auf die wirtschaftlichen Gewichte der Merkmale Fettmenge, Nutzungsdauer (ND) und Nettozunahme (NTZ) in $€$ je genetischer Standardabweichung im Referenzszenario bei Fleckvieh 
Abb. 4.17 Einfluss des Schlachtpreises für Mastbullen ( $€$ je kg SG) auf das wirtschaftliche Gewicht der Totgeburtenrate in $€$ je genetischer Standardabweichung im Referenzszenario bei Fleckvieh 86

Abb. 4.18 Grenznutzen ( \pm Standardabweichung) der einzelnen Merkmale in $€$ je Merkmalseinheit im Referenzszenario bei Braunvieh

Abb. 4.19

Wirtschaftliche Gewichte ( \pm Standardabweichung) der einzelnen Merkmale in $€$ je genetischer Standardabweichung im Referenzszenario bei Braunvieh

Abb. 4.20 Grenznutzen ( \pm Standardabweichung) der einzelnen Merkmale in $€$ je Merkmalseinheit im Vergleichsszenario bei Braunvieh.

Abb. $4.21 \quad$ Wirtschaftliche Gewichte ( \pm Standardabweichung) in $€$ je genetischer Standardabweichung im Vergleichsszenario bei Braunvieh

Abb. 4.22 Verhältnis der Merkmalskomplexe bei Braunvieh zueinander (\%) im Referenz- und im Vergleichsszenario ohne Quote.

Abb. 4.23 Vergleich der wirtschaftlichen Gewichte in $€$ je genetischer Standardabweichung der Rassen Holstein, Fleckvieh und Braunvieh in Szenarien mit aktuellem Milchauszahlungspreis und ohne Milchquotenbeschränkungen

Abb. 5.1

Einfluss der durchschnittlichen Nutzungsdauer in der Milchviehherde auf das wirtschaftliche Gewicht der Nutzungsdauer bei Holstein. 
Abkürzungsverzeichnis

$\mathrm{AKh}$

AUS

GZW

DFD

DMG

ECM

EKA

Ekg

Epro

EUROP

FECM

Fkg

Fla

FRUm

FRUp

Genet. Std.abw.

HKL

KVLm

KVLp

LG

MBK

MJ

ND

NEL

NTZ

PERS
Arbeitskraftstunde

Ausschlachtung

Gesamtzuchtwert

dark, firm, dry (dunkel, fest, trocken)

Durchschnittliches Minutengemelk

Energiekorrigierte Milch

Erstkalbealter

Eiweißmenge in $\mathrm{kg}$

Eiweißgehalt in \%

Handelsklasse für Fleisch

Fett- und Energiekorrigierte Milch

Fettmenge in $\mathrm{kg}$

Fleischanteil

Fruchtbarkeit maternal

Fruchtbarkeit paternal

genetische Standardabweichung

Handelsklasse

Kalbeverlauf maternal

Kalbeverlauf paternal

Lebendgewicht

Melkbarkeit

Megajoule

Nutzungsdauer

Nettoenergie Laktation

Nettozunahme

Persistenz 


$\begin{array}{ll}\text { PSE } & \text { pale, soft, exudative (blass, weich, wässrig) } \\ \text { RZE } & \text { Relativzuchtwert Exterieur } \\ \text { RZG } & \text { Relativzuchtwert Gesamt } \\ \text { RZN } & \text { Relativzuchtwert Nutzungsdauer } \\ \text { RZS } & \text { Relativzuchtwert Somatischer Zellgehalt } \\ \text { RZZ } & \text { Relativzuchtwert Zuchtleistung } \\ \text { SCS } & \text { Somatic Cell Score } \\ \text { TGR } & \text { Totgeburtenrate } \\ \text { TOTm } & \text { Totgeburtenrate maternal } \\ \text { TOTp } & \text { Totgeburtenrate paternal } \\ \text { TS } & \text { Trockensubstanz } \\ \text { TZ } & \text { Tägliche Zunahme } \\ \text { wG } & \text { wirtschaftliches Gewicht } \\ \text { XP } & \text { Rohprotein } \\ \text { ZKZ } & \text { Zwischenkalbezeit } \\ \text { ZZ } & \text { Zellzahl }\end{array}$




\section{$1 \quad$ Einleitung}

Der Gesamtzuchtwert in der Rinderzucht fasst die einzelnen Teilzuchtwerte zusammen und bietet damit ein Rangierungskritierium, das nicht nur die Milchleistung, sondern auch die funktionalen Merkmale entsprechend ihrer wirtschaftlichen Bedeutung berücksichtigt. In Zeiten der politischen und wirtschaftlichen Umstrukturierung in Europa und der europäischen Union wird es zunehmend wichtiger für die Landwirtschaft, die entsprechenden Weichen zu stellen und die Produktion an die zukünftigen Gegebenheiten anzupassen.

Wichtige Fragestellungen, die in den letzten Jahren zunehmend diskutiert wurden und noch immer werden, sind die Ausrichtung der Quotenpolitik und die Höhe des Milchauszahlungspreises. Die Milchproduzenten stehen vor der Entscheidung, wie es in ihren Betrieben weiter geht und brauchen Planungssicherheit, um handeln zu können. Die unsichere Quotenpolitik macht es nicht einfacher, mit dieser Situation umzugehen. Darüber hinaus ist der Milchauszahlungspreis unter großem Druck, die Milchpreise sinken in allen Teilen Deutschlands und die Kosten der Milchproduktion steigen an. Es wird seit Jahren prophezeit, dass die Milcherzeuger zu Weltmarktpreisen produzieren müssen, um überleben zu können, allerdings unter gehobenen europäischen Bedingungen und Auflagen.

Aus diesem Grund ist es für die einzelnen Zuchtverbände wichtig, sich für die zukünftigen Anforderungen zu rüsten und ihr Zuchtprogramm dementsprechend auszurichten. So ist die Entscheidung wichtig, welche Merkmale im Gesamtzuchtwert zu berücksichtigen sind und wie deren wirtschaftlicher Beitrag für den einzelnen Betrieb ist. Um diese Ziele in Einklang zu bringen, kann man den ökonomischen Nutzen der einzelnen Merkmale berechnen und sowohl die Genetik als auch die Wirtschaftlichkeit in die Zuchtwertschätzung einbeziehen. Es ist dadurch möglich, unter zukünftigen Bedingungen mit geeigneten Kühen Milch zu produzieren und den größtmöglichen Nutzen für die Kuh und den Landwirt zu erlangen.

Es scheint so, als verliere die Erhöhung der Milchmenge ihre dominierende Stellung im Zuchtziel, so dass andere Merkmale, welche die Produktionskosten senken, an Bedeutung gewinnen. International wird in der Zuchtwertschätzung vermehrt Gewicht auf die funktionalen Merkmale gelegt, um die Gesundheit und Nutzungsdauer der Kühe mehr in den Vordergrund zu stellen. Mit Hilfe des Gesamtzuchtwertes ist es möglich, den größtmöglichen Zuchtfortschritt aller Merkmale in Kombination zu erzielen und somit eine ausgeglichene Zucht zu ermöglichen. Deshalb werden die 
funktionalen Merkmale vermehrt stärker gewichtet, da in vielen Ländern die Fruchtbarkeit und Nutzungsdauer der Herden gering ist. Um dies zu ermöglichen, werden bei der Konzipierung des Gesamtzuchtwertes wirtschaftliche Gewichte verwendet, die über die ökonomische Seite der Merkmale Auskunft geben und diese entsprechend ihrer wirtschaftlichen Bedeutung charakterisieren. Aufgrund der vergangenen Entwicklungen in der Milchrinderzucht kann man annehmen, dass die funktionalen Merkmale weiterhin an Bedeutung zunehmen werden und dementsprechend stärker in einen Gesamtzuchtwert einfließen könnten.

Ziel der Arbeit ist es daher, wirtschaftliche Gewichte für die Rassen Holstein, Fleckvieh und Braunvieh abzuleiten, den Einfluss der ökonomischen und politischen Rahmenbedingungen zu untersuchen und Anregungen für die Zusammensetzung des Gesamtzuchtwertes zu geben. Dadurch soll eine Empfehlung gegeben werden, wie die Zucht in den kommenden Jahren auf zukünftige Bedingungen eingestellt werden kann. 


\section{Literaturübersicht}

Ziel der Tierzucht ist die Erhöhung der Wirtschaftlichkeit in der Erzeugung durch verbessertes Tiermaterial (Reinsch, 1993). Die wichtigste Komponente des züchterischen Fortschritts ist die Auswahl der besten Zuchttiere zur Erzeugung der nächsten Generation (Fewson, 1967). Um dieses Ziel in angemessener Weise für die gesamte Population zu erreichen, wurde ein Gesamtzuchtwert entwickelt, in dem alle züchterisch wichtigen Merkmale entsprechend ihrer wirtschaftlichen Bedeutung miteinander kombiniert werden. So wird gewährleistet, dass der höchstmögliche Zuchtfortschritt für die Gesamtpopulation erzielt wird.

Um die Merkmale entsprechend ihrer wirtschaftlichen Bedeutung im Gesamtzuchtwert zu berücksichtigen, werden wirtschaftliche Gewichte abgeleitet und bei der Indexselektion mit den Teilzuchtwerten, deren Sicherheiten und den genetischen Parametern der Merkmale kombiniert (Hazel und Lush, 1942).

\subsection{Ableitung wirtschaftlicher Gewichte}

Für die Berechnung eines Gesamtzuchtwertes ist die Kenntnis wirtschaftlicher Gewichte der einzelnen Teilzuchtwerte erforderlich. Der Gesamtzuchtwert $(\boldsymbol{T})$ entspricht der im Zuchtziel beschriebenen linearen Funktion aus relativen wirtschaftlichen Gewichten $\left(\boldsymbol{a}_{\boldsymbol{i}}\right)$ und Teilzuchtwerten $\left(\boldsymbol{u}_{\boldsymbol{i}}\right)$. In der Selektionsindextheorie ist der Gesamtzuchtwert üblicherweise als eine Funktion von zu verbessernden Merkmalen definiert, die jeweils mit ihrem wirtschaftlichen Gewicht multipliziert werden (Hazel, 1943).

$$
T=\sum_{i=1}^{m} a_{i} u_{i}
$$

In der Tierzucht werden die wirtschaftlichen Gewichte in Zuchtprogrammen eingesetzt, um die biologischen Merkmale gemäß ihrer ökonomischen Bedeutung zu charakterisieren (Habier et al., 2004). Das wirtschaftliche Gewicht zeigt nach Böbner (1994) in welchem Ausmaß der genetische Fortschritt in einem bestimmten Merkmal zur Verbesserung der Wirtschaftlichkeit beitragen kann. Miesenberger (1997) formuliert den Grenznutzen eines Merkmals als die Änderung des Gewinnes eines Unter- 
nehmers ( $\delta$ Gewinn), die sich aus der genetisch bedingten Produktionsänderung des betreffenden Merkmals um eine Einheit ergibt ( $\delta$ Merkmal), wenn alle übrigen Merkmale des Gesamtzuchtwertes konstant gehalten werden.

$$
\text { Grenznutzen }=\frac{\delta \text { Gewinn }}{\delta \text { Merkmal }}
$$

Um die Grenznutzen über die unterschiedlichen Merkmale vergleichbar zu machen, werden diese mit ihrer genetischen Standardabweichung $\left(\boldsymbol{\sigma}_{\boldsymbol{a}}\right)$ multipliziert.

$$
\text { wirtschaftlichesGewicht }=\text { Grenznutzen } \cdot \sigma_{a}
$$

Entscheidend für die Selektion nach dem Gesamtzuchtwert ist das Verhältnis der wirtschaftlichen Gewichte der einzelnen Merkmale zueinander, bezogen auf eine genetische Standardabweichung, und nicht deren absolute Höhe (Miesenberger, 1997). Die wirtschaftlichen Gewichte sind ein Mittel zur Gewinnmaximierung der Zucht. Es ist eine Verbesserung des Zuchtfortschritts möglich, allerdings ist dieses nur zu realisieren, wenn es ökonomisch auch sinnvoll erscheint. Zur Schätzung wirtschaftlicher Gewichte werden mathematischen Methoden verwendet (Böbner, 1994). Zum einen kann die Berechnung über eine partielle Ableitung nach dem beobachteten Merkmal erfolgen. Dies ist häufig mit der Verwendung von Gewinngleichungen verbunden (Brascamp et al., 1985; Smith et al., 1986). Zum anderen ist auch eine Berechnung über die Bestimmung der Differenz des Nettoertrages bei zwei verschiedenen Leistungsstufen möglich. In diesem Fall verwendet man nichtstetige Gewinngleichungen und bioökonomische Modelle (Groen et al., 1997).

Weitere grundsätzliche Punkte müssen bei der Ableitung von wirtschaftlichen Gewichten berücksichtigt werden:

Aus der Definition des wirtschaftlichen Gewichtes zeigt sich, dass nur die genetisch bedingte Leistungssteigerung in der Berechnung berücksichtigt werden darf (Melton et al., 1979; Böbner, 1994; Miesenberger, 1997; Vargas et al., 2002). Die Schätzung muss unter ceteri paribus Bedingungen erfolgen. Bei der Auswahl der Merkmale muss eine klare Auswirkung auf das Einkommen oder die Zielfunktion erkennbar sein (Pearson und Miller, 1981).

Es muss darauf geachtet werden, dass bei der Ableitung wirtschaftlicher Gewichte der gleiche Effekt nicht doppelt berücksichtigt wird (Dempfle, 1992; Mack, 1996; Weidele, 1996; Miesenberger, 1997; Nielsen, 2004). Eine Doppelberücksichtigung besteht dann, wenn ein Effekt zwei Merkmalen zugerechnet wird. Leitet man wirt- 
schaftliche Gewichte für das Merkmal somatische Zellzahl ab, so ist damit eine Leistungsdepression in der gesamten Laktation verbunden. Dieser Effekt wird allerdings schon dem wirtschaftlichen Gewicht der Milchleistung zugeordnet, so dass eine neuerliche Berücksichtigung im Merkmal somatische Zellzahl eine Doppelzählung gleichkommt. Es darf bei der Berechnung für dieses Merkmal lediglich die verworfene Milch im Behandlungszeitraum des Mastitisaufkommens berücksichtigt werden sowie die Tierarzt- und Medikamentenkosten und die zusätzliche Arbeitszeit (Nielsen, 2004).

In der Berechnung müssen zukünftige Produktionsbedingungen zugrunde gelegt werden, da das Zuchtziel in die Zukunft gerichtet ist und der Zuchtfortschritt erst in den Folgegenerationen realisiert werden kann. Deshalb sollte nach Weidele (1996) eine exakte Abbildung der Verhältnisse, wie sie für die Zukunft angenommen werden können, erfolgen. Da dies aufgrund der Voraussagekraft zukünftiger politischer und wirtschaftlicher Rahmenbedingungen sehr unsicher ist, können nur Annahmen über die Zukunft getroffen werden. Um diesen Unsicherheiten begegnen zu können, werden die Einflüsse unterschiedlicher Preis- und Marktsituationen untersucht.

Aus praktischen Gründen wird oft vom aktuellen Preisniveau, d.h. derzeitigen Erträgen und Kosten ausgegangen, da sich das Verhältnis der Merkmale zueinander über die Zeit weniger verändern als die absoluten Werte (Weidele, 1996). Allerdings erfolgt die Berechnung immer unter Einbeziehung verschiedener Szenarien. MundozLuna et al. (1988) empfehlen, die wirtschaftlichen Gewichte nur auf diejenige Rasse anzuwenden, für die eine Ableitung erfolgt. Die Übertragung auf eine andere Rasse ist nicht ohne weiteres möglich.

Die ermittelten wirtschaftlichen Gewichte werden anschließend in Form von Indices in die Zuchtwertschätzung einbezogen, um den maximalen Nutzen der züchterischen Arbeit zu erreichen (Hazel, 1943).

\section{2 Übersicht über die Methoden}

Zur Ableitung wirtschaftlicher Gewichte können prinzipiell zwei Ansätze verwendet werden: objektive und nicht-objektive Methoden. Von Rohr et al. (1999) geben zu bedenken, dass es nicht entscheidend ist, nach welcher Methode die wirtschaftlichen Gewichte berechnet werden, sondern dass es vielmehr wichtiger ist, dass die Modelle, mit denen die Berechnungen durchgeführt werden, korrekt definiert werden. Das Modell sollte abhängig von der Ausgangssituation und der vorliegenden Datengrundlage ausgewählt werden. 
Des Weiteren kann man zwischen deterministischen und stochastischen Ansätzen unterscheiden. Beim deterministischen Ansatz ist zu jedem Zeitpunkt geklärt, wie im Programm weiter verfahren werden soll, die Abläufe sind immer gleich. Beim stochastischen Ansatz liefert das Modell für gleiche Eingaben unterschiedliche Ausgaben, es handelt nach dem Zufallsmechanismus (Reinsch, 1993; Böbner, 1994).

\subsubsection{Nicht-objektive Methoden}

Die nicht-objektiven Methoden beruhen auf subjektiven Einschätzungen und Erfahrungswerten von Fachleuten und Konsumenten. Es gibt zwei grundsätzliche Unterscheidungen bei den nicht-objektiven Methoden: das subjektive Modell und den desired gains bzw. den restringierten Index.

\section{Subjektives Modell}

Ein subjektives Modell ist eine vereinfachte Methode zur Ableitung wirtschaftlicher Gewichte (Nielsen, 2004). Es wird verwendet, wenn es bei bestimmten Merkmalen schwierig ist, eine Berechnung auf einer objektiven Grundlage durchzuführen. Dabei werden nur die direkten Merkmale berücksichtigt, die den Betriebsgewinn unmittelbar beeinflussen. Indirekte Merkmale, die den Betriebsgewinn nicht direkt beeinflussen (z. B. funktionale Merkmale), werden gleich Null gesetzt. Dadurch kann es zu einem Rückgang der funktionalen Merkmale kommen, da diese nicht mit einem eigenen wirtschaftlichen Gewicht berücksichtigt werden, sondern nur über die genetischen Beziehungen zu den direkten Merkmalen. Nach Groen (1989a) sollte dieser Ansatz nur gewählt werden, wenn es für die einzelnen Merkmale schwierig ist, eine objektive Berechnung durchzuführen.

Edel und Dempfle (2004) verwenden den Ansatz einer kontingentierten Bewertung (Contingent Valuation) beim Süddeutschen Kaltblut, bei dem mithilfe von direkten Befragungstechniken die Zahlungsbereitschaft der Züchter und somit die Präferenz für eine Nutzenänderung in den Zuchtzielmerkmalen ermitteln. Die Contingent Value Studie ist eine anerkannte Methode, um komplexe öffentliche Güter zu bewerten, für die es keine ausreichende Marktbewertung gibt. Dabei soll der Befragte unter Berücksichtigung seiner wirtschaftlichen Rahmenbedingungen einen Preis nennen, bei dessen Bezahlung sein Nutzen derselbe wäre wie vor einer Nutzenänderung. Damit kann die Nutzenänderung als monetäre Größe beschrieben werden. 


\section{Desired Gains Index und restringierter Index}

Bei dem Desired Gains Index und dem restringierten Index ist es möglich, für jedes Merkmal einen erwünschten oder begrenzten genetischen Fortschritt vorzugeben (Brascamp, 1984). Anwendung finden diese vor allem in der Geflügel- oder Schweinezucht. Dabei werden wirtschaftliche Gewichte unter der Annahme eines erwünschten oder begrenzten Anteils an genetischem Zuwachs in die Berechnung eines Gesamtzuchtwertes einbezogen. Bei der Ableitung wirtschaftlicher Gewichte mithilfe des Desired Gains Index wird für zwei oder mehrere Merkmale der genetische Fortschritt vorherbestimmt und somit kann ein bestimmter Beitrag der Merkmale eigenständig vorherbestimmt werden.

Die Idee hinter dem restringierten Index ist es, dass der genetische Fortschritt eines oder mehrerer Merkmale auf Null begrenzt wird. Dieses erfolgt vor allem bei Merkmalen, für die es schwierig ist, ein wirtschaftliches Gewicht abzuleiten, bei denen aber eine Nichtberücksichtigung zu einer Verschlechterung des Merkmals käme (Reinsch, 1993). Das Merkmal wird dann so gewichtet, dass der genetische Fortschritt gleich Null ist, dabei aber die anderen Merkmale eine maximale Verbesserung erfahren. Als Beispiel gibt Reinsch (1993) die Fleischbeschaffenheit beim Schwein an (Häufigkeit von PSE- und DFD-Fleisch), da dieses Merkmal kaum Berücksichtigung in der Bezahlung findet, aber trotz allem einen entscheidenden Einfluss in der Züchtung hat.

\subsubsection{Objektive Methoden}

Die objektiven Methoden zeichnen sich dadurch aus, dass ein Modell mit Kosten und Erträgen aus der tierischen Produktion aufgebaut wird. Ein Modell ist eine Gleichung oder eine Reihe von Gleichungen mit Definitionen, Beschreibungen und Annahmen, welche das Verhalten im Produktionssystem widerspiegeln (Nielsen, 2004).

Es gibt zwei verschiedene Methoden, ein objektives Modell herzuleiten: den positiven und den normativen Ansatz. Das Ziel ist es, die tatsächlichen Strukturen durch ein modelliertes System wiederzugeben (Groen, 1989a).

\section{Positiver Ansatz}

Van Arendonk (1991) bezieht beim positiven Ansatz (Datenevaluation) zur Ableitung wirtschaftlicher Gewichte in die Modelle Daten ein, die unter aktuellen Produktionsbedingungen erfasst worden sind. Allerdings bietet dieser Ansatz nicht die Möglichkeit, den Einfluss von veränderten Produktionsbedingungen oder Preisen auf die 
wirtschaftlichen Gewichte zu hinterfragen. Da nur aktuelle Preise in die Berechnung eingehen, erscheint sie nach Groen (1989a) weniger geeignet für die Darstellung zukünftiger Entwicklungen zu sein. Eigentlich sind die im Modell zu verwendeden Preise so zu wählen, dass sie die Situation zum Zeitpunkt der genetischen Verbesserung erfassen.

Für diese Art der Ableitung wird ein großer Datensatz benötigt, der jedoch oft nicht verfügbar ist, da ein großer Teil der benötigten Daten schwer erfassbar oder nicht verfügbar ist (Nielsen, 2004). Reinsch (1993) zeigt, dass der positive Ansatz kaum Bedeutung in der Praxis hat.

\section{Normativer Ansatz}

Im Gegensatz zum positiven Ansatz bietet der normative Ansatz (Datensimulation) die Möglichkeit, zukünftige Annahmen in das Modell mit einzubeziehen (van Arendonk, 1991). Das Modell dient dazu, ein Produktionssystem zu simulieren. Um allerdings eine ökonomische Leistung zu simulieren, müssen alle berücksichtigten Merkmale und ihre Beziehungen zueinander bekannt sein. Gerade bei funktionalen Merkmalen gestaltet sich dies schwierig, da für diese nicht alle benötigten Informationen vorliegen.

Der normative Ansatz ist in einzelne Modelle unterteilt. Im Allgemeinen kommen in der Tierzucht Gewinngleichungen oder bioökonomische Modelle zur Anwendung.

\section{Gewinngleichungen}

Die Gewinngleichung ist eine einfache und übersichtliche Methode zur Berechnung der wirtschaftlichen Gewichte und wurde in der Vergangenheit vielfach zur Ableitung herangezogen. Es handelt sich um ein Ein-Gleichungs-Modell (Nielsen, 2004).

In der Tierzucht ist das Modell der Gewinngleichungen von Moav und Moav (1966) eingeführt worden. Wie bei Böbner (1994) dargestellt, kann man den Grenznutzen eines Merkmals auf zwei verschiedenen Arten berechnen. Zunächst ist die Berechnung über die partielle Ableitung der Gewinngleichung nach dem entsprechenden Merkmal möglich. Nach dieser Methode wurden die wirtschaftlichen Gewichte bei Smith (1985) berechnet, was besonders bei der Ableitung wirtschaftlicher Gewichte für Hauptmerkmale zur Anwendung kommt. Die andere Variante der Berechnung ist die Veränderung eines bestimmten Merkmals in der Gleichung unter Konstanthaltung aller übrigen Variablen. Durch die Quantifizierung der dadurch entstandenen Differenz im Nettoertrag kann eine Berechnung erfolgen. Nach Miesenberger (1997) muss die Gleichung, bezogen auf die Ableitung der wirtschaftlichen Gewichte, differen- 
zierbar sein und die Beziehungen dürfen nicht zu komplex sein, damit sie in angemessener Weise in der Gleichung berücksichtig werden können. Ziel dabei ist es, aus der Ermittlung der Differenz von Ertrag und Aufwand, den Gewinn einer Merkmalsveränderung abzuleiten. Brascamp et al. (1985), Smith et al. (1986) und Gibson (1989b) entwickeln die Gewinngleichungen für komplexere Situationen weiter, indem sie u. a. Quotensituationen mit berücksichtigen. Das Modell der Gewinngleichung kann die Wirtschaftlichkeit eines Milchviehbetriebes bzw. einer Kuh nicht im Detail beschreiben (Nielsen, 2004). Die Aussagekraft, die man bei der Berechnung wirtschaftlicher Gewichte erreicht, ist stark abhängig von der Genauigkeit und der Vollständigkeit der einbezogenen Parameter (Böbner, 1994).

\section{Lineare Programmierung}

Die Methode der Linearen Programmierung wird schon seit langer Zeit in anderen Wirtschaftsbereichen angewandt (Miesenberger, 1997). Man versucht mit einem vereinfachten Modell die Wirklichkeit so realistisch wie möglich abzubilden (Reisch, 1962). Dieses Modell bietet den Vorteil, dass in Situationen mit beschränkt zur Verfügung stehenden Ressourcen der maximale Gewinn abgeleitet werden kann. Die Lineare Programmierung ist ein Verfahren, um für ein lineares Gleichungssystem, das bestimmten Nebenbedingungen unterworfen ist, eine Extremwertlösung (Minimum oder Maximum) zu finden (Weinschenck, 1964).

Bei der Linearen Programmierung werden systematische und optimale Lösungen von Gleichungen und Ungleichungen ermittelt, die auf Grundsätze der Matrixalgebra und Vektorenanalyse zurückgreifen. Das Modell zeichnet eine hohe Leistungsfähigkeit aus, d. h. es kann mit vielen Gleichungen und Variablen gerechnet werden (Reisch, 1962). Bei dem Aufbau des Modells mit linearen Produktionsfunktionen und $\mathrm{Zu}-$ sammenhängen können Effekte auf Leistungen, Fütterung, Futterbewertung, Arbeitswirtschaft, Struktur der Flächennutzung und Viehhaltung berücksichtigt werden. Nach Reisch (1962) gibt es drei wichtige Punkte bei der Erstellung des Modells: Zum einen ist eine Zielfunktion zu definieren. Zum anderen müssen Gleichungen aufgestellt werden, welche die für das Programm in Frage kommenden Aktivitäten bzw. Prozesse durch die Produktionskoeffizienten und den Nettoertragswert charakterisieren (Produktions- und Kostengleichung). Außerdem sind Beschränkungsund/oder Bedingungsgleichungen bzw. - ungleichungen zu berücksichtigen, die angeben, welche Produktionsmittel in bestimmter begrenzter Menge verfügbar sind. Böbner (1994) weist darauf hin, dass dieser Methode eine hohe Komplexität zu Grunde liegt. Reisch (1962) gibt zu bedenken, dass die Ergebnisse immer nur so gut sein können, wie das Modell die zu untersuchende Situation wiedergibt. 


\section{Dynamische Programmierung}

Die Dynamische Programmierung ist ein mathematisches Verfahren zur Entscheidungsfindung bei einer Folge voneinander abhängiger Entscheidungen (Böbner, 1994). Es ist ein Ansatz, um aufeinander folgende oder mehrstufige Entscheidungsprobleme zu lösen. Dabei wird ein mehrstufiges Problem gelöst, indem eine Reihe von Einzelproblemen betrachtet wird. Diese systematische Vorgehensweise optimiert die Effektivität des Gesamtsystems bei kombinierten Entscheidungen. Bisher wurde diese Methode in der Tierzucht vor allem zur Bestimmung von optimalen Remontierungs- oder Merzungszeitpunkten angewandt (Böbner, 1994).

Reisch (1962) beschreibt die Dynamische Programmierung als endliches Modell mit Zeitveränderung. Ziel soll es sein, nicht den Gewinn in der einzelnen Periode zu maximieren. Man strebt nicht den Betriebserfolg für einen bestimmten Zeitpunkt an, sondern über einen längeren Zeitraum hinweg.

Des Weiteren werden die meisten Programmierungsverfahren durchgeführt, als hätte man genaue Kenntnisse von den zukünftigen wirtschaftlichen Geschehnissen. Daraus resultieren eine große Unsicherheit und ein Risiko für die Berechnung. Diese Methode benötigt kurze Rechenzeiten bei einfachen Problemen, für komplexere Fragestellungen müssen allerdings Zwischenlösungen vorgegeben werden (Weidele, 1996).

\subsection{Ergebnisse zur Schätzung wirtschaftlicher Gewichte}

Die Ableitung wirtschaftlicher Gewichte wird schon seit langem in der nationalen und internationalen Tierzucht diskutiert. Allerdings ist keine Vergleichbarkeit der Ergebnisse aus den verschiedensten Ländern und Regionen gegeben. Reinsch (1993) betont, dass die Gewichtungsfaktoren aus verschiedenen Arbeiten nicht miteinander kombiniert werden können, um einen einheitlichen Zuchtwert zu realisieren. Dies beruht darauf, dass die Arbeiten zu diesem Thema in unterschiedlichen Ländern und Regionen durchgeführt wurden und jeweils unterschiedliche Rahmenbedingungen vorherrschen. Zusätzlich wurden unterschiedliche Merkmale betrachtet und diese in manchen Fällen noch in anderer Weise definiert. Eine weitere Ursache für die mangelnde Kompatibilität sind die unterschiedlichen ökonomischen Modelle, mit denen in den Untersuchungen gearbeitet wird.

Zunächst sind jüngere Arbeiten aus europäischen Ländern dargestellt und im Anschluss wird ein allgemeiner Überblick über die Ergebnisse zur Ableitung wirtschaftlicher Gewichte gegeben. 


\subsubsection{Dänemark}

Das Produktionsniveau der dänischen Milchpopulation kann in einer Simulation von Nielsen (2004) erfolgreich erhöht werden. Allerdings kommt es dabei zu unerwünschten Nebeneffekten wie Krankheiten und Reproduktionsproblemen, da zunächst der Schwerpunkt der Betrachtung auf die Produktionsmerkmale gelegt wird. Deshalb soll es nach Nielsen (2004) zu einer ausbalancierten Verbesserung von Produktions- und funktionalen Merkmalen kommen. Dies wird seit einigen Jahren in Dänemark im Zuchtprogramm und in der Zuchtwertschätzung berücksichtigt.

Nielsen (2004) leitet die wirtschaftlichen Gewichte unter dänischen Bedingungen mit dem SimHerd-Modell von Østergaard et al. (2000; 2003) und Sørensen et al. (1992) ab. Im Modell wird ein normativer Ansatz verwendet, in dem das Produktionsumfeld detailliert beschrieben wird. Anhand eines dynamischen Modells wird die Leistung der Herde simuliert. Zwar erfolgen die Berechnungen im Modell auf Basis der Einzelkuh, allerdings sind die phänotypischen Auswirkungen wieder auf Basis der Herde beschrieben (Sørensen et al., 1992). Ein Tier definiert sich zu einem bestimmten Zeitpunkt über das Alter, das Laktationsstadium, die Laktationsnummer, die aktuelle Lebendmasse, die Milchleistung, den Trächtigkeitsstatus und das Krankheitsvorkommen (Nielsen, 2004). Für die Remontierung werden alle Färsen des Betriebes herangezogen. Die Einstellung der Färse erfolgt, wenn die aktuelle Anzahl der Kühe niedriger ist als die maximale Herdenkapazität oder wenn mindestens eine Kuh aus der Herde zur Merzung ausgewählt ist. Ein Zukauf ist dann nötig, wenn keine Färsen zum benötigten Zeitpunkt verfügbar sind. Sind zu viele Färsen zur Remontierung vorhanden, werden die überzähligen verkauft. Die Herdendynamik entsteht durch das Einsetzen und Merzen in und aus der Herde und durch den Wechsel in den Kuhgruppen durch Kalbung. Die Merzungsstrategie wird durch die kontrollierte Herdengröße mit einer maximalen Anzahl Kühe in der Herde, durch die Festsetzung des Zeitpunktes, an dem ein Tier besamt bzw. trächtig sein muss, und durch die Milchleistung bestimmt (Nielsen, 2004). Als Grundlage der Berechnung von Nielsen (2004) dient ein durchschnittlicher dänischer Betrieb mit einer Betriebsgröße von 70 Kühen plus Nachzucht.

In Tab. 2.1 sind die Grenznutzen für zehn Merkmale dargestellt. Die Ableitung erfolgt durch einen Vergleich der Ausgangssituation und dem dargestellten Szenario. Die wirtschaftlichen Gewichte im ersten Szenario werden abgeleitet, indem jedes Merkmal bei der Ableitung um 20\% verändert wird. Das zweite Szenario zeigt ausschließlich die aktuellen Produktionsbedingungen und die Ableitung erfolgt auf Höhe des üblichen genetischen Fortschritts. 
Tab. 2.1 Grenznutzen in $€$ je Merkmalseinheit pro Kuh und Jahr für Produktionsund funktionale Merkmale in einem Szenario bei einer Merkmalsverbesserung von jeweils 20\% in allen Merkmalen und in einem Szenario mit aktuellen Produktionsbedingungen (nach Nielsen, 2004)

\begin{tabular}{lccc}
\hline & Einheit & $\begin{array}{c}20 \% \text { genetische Ver- } \\
\text { änderung in jedem } \\
\text { Merkmal }\end{array}$ & $\begin{array}{c}\text { Aktuelle Produkti- } \\
\text { onsbedingungen }\end{array}$ \\
\hline ECM & kg & 0,28 & 0,13 \\
Konzeptionsrate & Tage & 1,98 & 1,69 \\
Tage bis zur ersten Besa- & \% & $-0,94$ & $-0,62$ \\
mung & Häufigkeit & $-162,50$ & $-0,61$ \\
Totgeburtenrate & Häufigkeit & $-79,00$ & $-124,00$ \\
Mastitis & Häufigkeit & $-402,10$ & $-39,00$ \\
Plazenta & Häufigkeit & $-210,20$ & $-277,00$ \\
Milchfieber & kg & $-0,76$ & $-127,00$ \\
Laminitis & $\%$ & $-6,66$ & $-0,51$ \\
Lebendmasse & & & $-5,20$ \\
Unfreiwillige Merzung & &
\end{tabular}

In der ersten Variante werden die Merkmale um eine genetische Variation von $20 \%$ verändert. Nielsen (2004) wählt diese hohe Variation aufgrund des instabilen Grenznutzens der Krankheitsmerkmale bei hohen Standardabweichungen. In den verschiedenen von Nielsen (2004) betrachteten Szenarien unterscheiden sich die Grenznutzen in den funktionalen Merkmalen nicht wesentlich voneinander. Dies ist dadurch $\mathrm{zu}$ erklären, dass die unterschiedlichen Preise in den Szenarien nur wenig Einfluss auf den Ertrag und die Kosten nehmen. Die größten Unterscheide ergeben sich durch die Berücksichtigung eines Quotenszenarios, da sich die relative wirtschaftliche Gewichtung der Milchleistung im Vergleich zu den funktionalen Merkmalen verändert. Die Grenznutzen sind zudem unter der Rahmenbedingung Milchquote geringer als in einer Situation mit festgelegter Kapazität an Kuhplätzen (Gibson, 1989a; Groen, 1989a; Veerkamp et al., 2002; Nielsen, 2004). 
Nielsen (2004) stellt fest, dass ein ansteigendes genetisches Niveau der Milchleistung $\mathrm{zu}$ einem höheren Betriebsgewinn führt. Wegen der indirekten Effekte zwischen Milchleistung, Futteraufnahme und Körpergewicht kommt es bei ansteigendem Lebendgewicht jedoch zu einer Reduktion der Milchleistung.

\subsection{2 Österreich}

Miesenberger (1997) stellt anhand eines Simulationsprogramms von Amer et al. (1994) eine Rinderherde mit den Bereichen Milchviehhaltung, Färsenaufzucht und Bullenmast bei unterschiedlichen Rassen in einem statischen Zustand über einen unendlichen Planungshorizont dar. Die von Miesenberger (1997) berechneten wirtschaftlichen Gewichte sind in Tab. 2.2 dargestellt.

Tab. 2.2 Wirtschaftliche Gewichte für Produktions- und funktionale Merkmale in $€$ je genetischer Standardabweichung bei der Rasse Fleckvieh (nach Miesenberger, 1997)

\begin{tabular}{lccc}
\hline \multicolumn{1}{c}{ Merkmal } & Einheit & genet. Std.abw. & $€$ /genet. Std.abw. \\
\hline Milchträger & $\mathrm{kg}$ & 350 & 0 \\
Fett & $\mathrm{kg}$ & 15 & 16,35 \\
Eiweiß & $\mathrm{kg}$ & 11 & 19,99 \\
Nutzungsdauer & $\mathrm{Tag}$ & 180 & 22,24 \\
Konzeptionsrate & $\%$ & 5 & 7,27 \\
Persistenz & $\sigma$ & 1 & 2,91 \\
Kalbeverlauf & $\mathrm{Klasse}$ & 0,22 & 1,74 \\
Totgeburtenrate & $\%$ & 2,5 & 4,00 \\
Tgl. Zunahme & $\mathrm{g}$ & 47 & 11,26 \\
EUROP & Klasse & 0,25 & 4,22 \\
Ausschlachtung & $\%$ & 1,14 & 11,26 \\
\hline
\end{tabular}


Bei der Rasse Fleckvieh wird ein positives wirtschaftliches Gewicht für die Nutzungsdauer nicht durch eine höhere Milchleistung realisiert, sondern resultiert hauptsächlich aus verringerten Remontierungskosten und steigenden Erlösen aus dem Verkauf von überzähligen Färsen.

Im Gegensatz dazu wurde ein wesentlich höheres wirtschaftliches Gewicht für das Merkmal Nutzungsdauer bei der Rasse Schwarzbunt realisiert. Dieses ergibt sich aus einer größeren Differenz zwischen Kosten der Bestandsergänzung und Erlösen aus der Merzung von Kühen. Anhand der von Miesenberger (1997) zusätzlich durchgeführten Sensitivitätsanalysen wird deutlich, dass die Unterschiede in den Grenznutzen zwischen den Rassen hauptsächlich durch die unterschiedlichen Annahmen in der Preissituation hervorgerufen werden. Besonders der Kraftfutterpreis wirkt sich nachhaltig auf die wirtschaftlichen Gewichte aus.

\subsubsection{Deutschland}

Weidele (1996) und Mack (1996) berechnen Grenznutzen je Merkmalseinheit mit der Methode der Linearen Programmierung. Mack (1996) leitet die Grenznutzen für den landwirtschaftlichen Einzelbetrieb mit den Rassen Schwarzbunte und Fleckvieh ab, wohingegen Weidele (1996) eine sektorale Betrachtung vornimmt.

\section{Einzelbetrieblicher Ansatz}

Die von Mack (1996) abgeleiteten Grenznutzen je Merkmalseinheit werden mit einem simultan-dynamischen Optimierungsmodell für einen Futterbaubetrieb berechnet. Die allgemeine Betriebsentwicklung gestaltet sich nach den Untersuchungen von Mack (1996) problematisch. Wenn in Betrieben mit der Rasse Fleckvieh keine Milchquote gepachtet werden kann, geht mit einer Leistungssteigerung automatisch eine Bestandsreduktion einher. Verändern sich die endogenen Faktoren, hat dies Einfluss auf die Aufstellung des Betriebes. Kommt es zur Milchleistungssteigerung, nehmen die Futterkosten zu. Zusätzlich ist ein Mehrbedarf an Arbeitszeit nötig und Grünflächen werden freigesetzt. Wird die gesteigerte Milchleistung auf eine Verringerung im Erstkalbealter zurückgeführt, gehen auch die Arbeitszeit und der Bedarf an Grünland in der Jungviehaufzucht zurück. Liegt eine Begrenzung durch das Milchkontingent vor, erscheint lediglich eine Steigerung der Eiweißmenge sinnvoll. Allerdings ist eine Verbesserung dieses Merkmals im Wesentlichen abhängig von den betriebswirtschaftlichen Rahmenbedingungen. Die Grenznutzen der einzelnen Merkmale für Schwarzbunte sind in Tab. 2.3 aufgeführt (Mack, 1996). Beim Rassenvergleich fällt auf, dass die Grenznutzen für die Leistungsmerkmale bei der Rasse Schwarzbunt niedriger sind als bei der Rasse Fleckvieh. 
Tab. 2.3 Grenznutzen der Leistungs- und funktionalen Merkmale für Schwarzbunte unter alternativen wirtschaftlichen Rahmenbedingungen in $€$ je Merkmalseinheit (nach Mack, 1996)

\begin{tabular}{lccccc}
\hline Merkmal & Einheit & $\begin{array}{c}\text { Milchquoten- } \\
\text { zupacht }\end{array}$ & $\begin{array}{c}\text { Milchquote } \\
\text { fix }\end{array}$ & $\begin{array}{c}\text { Ab- } \\
\text { wanderung } \\
\text { AK }\end{array}$ & $\begin{array}{c}\text { Einsparung } \\
\text { Stallplätze }\end{array}$ \\
\hline Milch & $\mathrm{kg}$ & 0,08 & 0,03 & 0,07 & 0,09 \\
Fett & $\mathrm{kg}$ & 0,72 & 0,30 & 0,69 & 11,10 \\
Eiweiß & $\mathrm{kg}$ & 2,78 & 2,96 & 2,80 & 2,79 \\
TS-Aufnahme & $\mathrm{kg}$ & 77,96 & 69,89 & 68,33 & 77,50 \\
Nutzungsdauer & Tage & 44,61 & 22,65 & 72,82 & 23,03 \\
Totgeburtenrate & $\%$ & 2,71 & 1,65 & 3,40 & 2,40 \\
Kalbeverlauf & Klasse & 0,40 & 0,28 & 0,52 & 0,26 \\
ZKZ & Tage & $-0,85$ & $-0,48$ & $-0,59$ & $-1,17$ \\
EKA & Monat & 8,84 & 7,89 & 11,81 & 6,60 \\
\hline
\end{tabular}

\section{Sektoraler Ansatz}

Weidele (1996) leitet Grenznutzen auf der sektoralen Ebene ab, um eine Ergänzung $\mathrm{zu}$ den vielfältig durchgeführten einzelbetrieblichen Betrachtungen zu geben. In Form von Gruppenhöfen werden die Auswirkungen der Merkmalsveränderungen auf die sektoralen Input-Output-Relationen untersucht und die Auswirkungen der Landwirtschaft auf andere Wirtschaftsbereiche in Deutschland betrachtet.

Es zeigen sich schon bei geringen Leistungssteigerungen strukturelle Veränderungen (Weidele, 1996). Unter allen betrachteten Szenarien kommt es zu einem Abbau des gesamten Kuhbestandes aufgrund von Milchleistungssteigerungen. Die Verbesserung der funktionalen Merkmale hingegen hat unterschiedliche Auswirkungen. Die Variation des Merkmals Nutzungsdauer hat nachhaltigen Einfluss auf die Struktur der Betriebe, da alle Bereiche der Milchviehhaltung in gleichem Maße betroffen sind (Weidele, 1996). In Tab. 2.4 sind die Grenznutzen für Leistungs- und funktionale Merkmale für Schwarzbunte dargestellt. 
Tab. 2.4 Grenznutzen der Leistungs- und funktionalen Merkmale für Schwarzbunte in $€$ je Merkmalseinheit unter verschiedenen Szenarien (nach Weidele, 1996)

\begin{tabular}{lcccc}
\hline \multicolumn{1}{c}{ Merkmal } & $\begin{array}{c}\text { Merkmals- } \\
\text { intervall }\end{array}$ & $\begin{array}{c}\text { Optimistische } \\
\text { Erwartungen }\end{array}$ & $\begin{array}{c}\text { Pessimistische } \\
\text { Erwartungen }\end{array}$ & $\begin{array}{c}\text { Weitere } \\
\text { Preis- } \\
\text { senkungen }\end{array}$ \\
\hline FECM & $+100 \mathrm{~kg}$ & 0,014 & 0,01 & 0,02 \\
Fett & $+0,1 \%$ & $-0,99$ & $-1,13$ & $-0,79$ \\
Eiweiß & $+0,1 \%$ & 2,96 & 0,60 & 2,44 \\
EKA & -3 Monate & 4,60 & 4,60 & 4,46 \\
ZKZ & +20 Tage & $-0,63$ & $-0,64$ & 0,57 \\
Nutzungsdauer & $+0,25 \mathrm{Jahre}$ & 26,50 & 26,50 & 30,61 \\
Totgeburtenrate & $-1 \%$ & 5,76 & 5,59 & 5,25 \\
Kalbeverlauf & $+1 \%$ & 1,19 & 0,50 & 0,50 \\
TS-Aufnahme & $+1 \mathrm{~kg} \mathrm{TS} /$ Tag & 55,35 & 53,30 & 56,54 \\
\hline
\end{tabular}

Weidele (1996) stellt heraus, dass die Milchleistungsmerkmale Milchmenge und Eiweißprozente in allen Szenarien überragende Bedeutung haben. In diesem Modell würden erst bei einem Milchpreis unter 0,204 $€ / \mathrm{kg}$ Milch vermehrt Milchviehhalter aus der Produktion ausscheiden, da es an Alternativen der Beschäftigung mangelt. Allerdings gewinnen auch die funktionalen Merkmale an Bedeutung.

Als Fazit führt Weidele (1996) einen Vergleich zwischen einzelbetrieblichem und sektoralem Grenznutzen durch. Dabei fällt auf, dass der Nutzungsdauer in den beiden Modellen eine unterschiedliche Bewertung zukommt. Weiterhin weist Weidele (1996) darauf hin, dass der Schwerpunkt der Gewichtung auch in Zukunft bei den Leistungsmerkmalen zu sehen ist. Allerdings sollte die Fettleistung, entgegen der Berechnungen, nicht negativ im Gesamtzuchtwert berücksichtigt werden, die Vorrangstellung der Eiweißmenge sollte jedoch herausgestellt werden. Da die angenommenen Rahmenbedingungen die Grenznutzen in hohem Maße beeinflussen, ist es wichtig, das Modell so realistisch wie möglich zu gestalten (Weidele, 1996). Des Weiteren sollten zur Erstellung eines Gesamtzuchtwertes sowohl einzelbetriebliche als auch sektorale Berechnungen zu Rate gezogen werden, um die Einbindung falscher Markttendenzen zu vermeiden. 
Zusammenfassend schlussfolgern Mack et al. (1997), dass sich die Grenznutzen für die Milchleistungsmerkmale zwischen Holstein und Fleckvieh nur geringfügig voneinander unterscheiden. Zudem ist der Nutzen einer Milchleistungssteigerung stark abhängig von dem unterstellten Szenario mit oder ohne Milchquotenbeschränkung. Eine Verbesserung der Zuchtleistung ist in allen Szenarien weiterhin wirtschaftlich.

\subsubsection{Schweiz}

Böbner (1994) leitet wirtschaftliche Gewichte unter schweizerischen Bedingungen mit Hilfe der stochastischen Dynamischen Optimierung ab. Damit können besonders einzelne Managemententscheidungen hinsichtlich der vorgegebenen Zielfunktion optimiert werden.

Nach Reinsch (1993) sollte eine Schätzung der wirtschaftlichen Gewichte von funktionalen Merkmalen nur in Zusammenhang mit Produktionsmerkmalen stattfinden. Allerdings ist die Dynamische Programmierung bei Böbner (1994) nur auf die Ableitung bei funktionalen Merkmalen ausgerichtet. Als Vergleichswert wird jedoch ein wirtschaftliches Gewicht für das Merkmal Milchleistung berechnet. Die von Böbner (1994) abgeleiteten Merkmale sind in Tab. 2.5 dargestellt.

Tab. 2.5 Berechnung der wirtschaftlichen Gewichte in $€$ je genetischer Standardabweichung in der Schweiz (nach Böbner, 1994)

\begin{tabular}{lcc}
\hline Merkmal & Einheit & $€ /$ Standardabweichung \\
\hline Milchleistung & $\mathrm{kg}$ & 115,30 \\
Konzeptionsrate & $\%$ & 37,20 \\
Grundfutteraufnahmevermögen & $\mathrm{kg}$ & 36,13 \\
Nutzungsdauer & Tage & 28,90 \\
Schwergeburten & $\%$ & 12,30 \\
Persistenz & $\sigma$ & 12,50 \\
\hline
\end{tabular}

Das wichtigste Merkmal in der Modellannahme der Referenzlösung ist nach der Berechnung von Böbner (1994) die Milchleistung. Die relative Bedeutung der Merkmale Schwergeburt und Konzeptionsrate zur Milchleistung liegen bei 10,7\% bzw. $32,2 \%$. Mit steigendem Leistungsniveau sinkt das wirtschaftliche Gewicht der Milchleistung und die wirtschaftlichen Gewichte der Sekundärmerkmale steigen an. Die 
Sensitivitätsanalysen zeigen eine deutliche Abhängigkeit der wirtschaftlichen Gewichte vom unterstellten Milchleistungsniveau.

Böbner (1994) gibt zu bedenken, dass es starke saisonale Schwankungen in den wirtschaftlichen Parametern wie Milchpreis, Futterkosten und Erlöse für Verkaufstiere gibt. Diese haben durchaus Einfluss auf die erwirtschafteten Erträge, sind aber schwer in der Berechnung zu berücksichtigen. Ein weiteres Problem in der Ableitung wirtschaftlicher Gewichte stellen die Opportunitätskosten dar. Mittels Dynamischer Programmierung bzw. bewerteten Markov-Prozessen (Reinsch, 1993) ist es möglich, auch die Opportunitätskosten zu berücksichtigen. Allerdings zeigt van Arendonk (1991), dass die Opportunitätskosten in seiner Studie keinen Einfluss auf das Hauptleistungsmerkmal Milchmenge haben. Den Verwendungszweck seiner Ergebnisse sieht Böbner (1994) als Nutzen für die züchterische Arbeit und zur ökonomischen Beurteilung von Managemententscheidungen innerhalb eines Betriebes. Züchterischen Nutzen versprechen nach Dempfle (1992) und Reinsch (1993) die Merkmale Eutergesundheit, Stoffwechselerkrankungen und Probleme des Bewegungsapparates.

Allerdings leiten Baumung und Sölkner (1998) erneut wirtschaftliche Gewichte unter schweizerischen Bedingungen ab. Die Berechnungen basieren auf dem Programm von Miesenberger (1997). Es werden wirtschaftliche Gewichte für die Merkmale Milchträger, Fettmenge, Eiweißmenge, Nutzungsdauer, Konzeptionsrate, Persistenz, Kalbeverlauf und Totgeburtenrate abgeleitet (Tab. 2.6). Bei der Rasse Fleckvieh werden zusätzlich die Fleischleistungsmerkmale Nettotageszunahme und Handelsklasse betrachtet. 
Tab. 2.6 Übersicht über die wirtschaftlichen Gewichte bei Holstein und Fleckvieh in der Schweiz ohne Milchquotenbeschränkung (Baumung und Sölkner, 1998)

\begin{tabular}{|c|c|c|c|c|c|}
\hline \multirow[b]{2}{*}{ Merkmal } & \multirow[b]{2}{*}{ Einheit } & \multicolumn{2}{|c|}{ Holstein } & \multicolumn{2}{|c|}{ Fleckvieh } \\
\hline & & $\mathrm{s}_{\mathrm{A}}$ & wG & $\mathrm{s}_{\mathrm{A}}$ & wG \\
\hline Milchträger & $\mathrm{kg}$ & 434 & 65,10 & 400 & 52,00 \\
\hline Fettmenge & $\mathrm{kg}$ & 18 & 31,86 & 116 & 27,36 \\
\hline Eiweißmenge & $\mathrm{kg}$ & 12 & 79,68 & 12 & 84,36 \\
\hline Nutzungsdauer & Tag & 150 & 54,00 & 150 & 52,50 \\
\hline Konzeptionsrate & $\%$ & 5 & 8,60 & 5 & 11,15 \\
\hline Persistenz & $\%$ & 3 & 14,13 & 3 & 15,54 \\
\hline Kalbeverlauf & Klasse & 0,22 & 2,32 & 0,22 & 2,73 \\
\hline Totgeburtenrate & $\%$ & 2,5 & 2,58 & 2,5 & 4,88 \\
\hline Nettotageszunahme & g & - & - & 30 & 10,20 \\
\hline Handelsklasse & Klasse & - & - & 0,25 & 15,34 \\
\hline
\end{tabular}

Im Gegensatz zu Böbner (1994) sind die wirtschaftlichen Gewichte für die Milchleistung niedriger, allerdings sind diese auch in die Einzelmerkmale Milchträger, Fettmenge und Eiweißmenge aufgeteilt (Tab. 2.6). Dagegen ist das wirtschaftliche Gewicht für die Nutzungsdauer höher, die wirtschaftlichen Gewichte der anderen vergleichbaren Merkmale sind allerdings geringer.

Baumung und Sölkner (1998) leiten wirtschaftliche Gewichte zusätzlich unter schweizerischen Annahmen und einer Beschränkung der Milchmengenablieferung ab. Dabei fällt auf, dass sich lediglich das wirtschaftliche Gewicht des Milchträgers verändert. Unter Milchmengenbeschränkungen ist das Gewicht geringer. Es zeigt sich des Weiteren, dass in Szenarien mit Quote die Bedeutung der funktionalen Merkmale gegenüber den Leistungsmerkmalen zunimmt. 


\subsubsection{Weitere Arbeiten zu wirtschaftlichen Gewichten}

Das Ziel der Arbeit von Kulak et al. (2003; 2004) ist die Berechnung und der Vergleich von wirtschaftlichen Gewichten in den skandinavischen Ländern. Die Unterschiede, die zwischen den Ländern bei der Ableitung wirtschaftlicher Gewichte auftreten, sind auf die unterschiedlichen Produktionsumstände in den einzelnen Ländern zurückzuführen. Das höchste wirtschaftliche Gewicht für die Milchproduktion hat Finnland. Dies beruht auf dem hohen Milchpreis von $0,47 € / \mathrm{kg}$ Milch in diesem Land. Der Rückgang von Krankheiten hat durch die Reduktion von Behandlungskosten in allen Ländern einen positiven Effekt auf den Betriebsgewinn. Eine verlängerte Nutzungsdauer führt zu einer größeren Anzahl von Kühen in den hochleistenden Altersklassen. Es kommt in Verbindung damit zu geringeren Remontierungskosten und einer positiven Veränderung im Betriebsgewinn. Kulak et al. (2004) stellen fest, dass die Milchquote auf die meisten berücksichtigten Merkmale einen Einfluss hat. Besonders in Finnland ist der Einfluss auf die wirtschaftlichen Gewichte am Größten.

Dekkers (1991) schätzt wirtschaftliche Gewichte unter suboptimalen Managementstrategien. Es zeigt sich, dass das Ausmaß der Veränderung der Merkmale bei der Berechnung der Gewichte wichtig ist. Veränderte Merzungs- oder Managementstrategien haben einen geringeren Einfluss auf die Höhe der wirtschaftlichen Gewichte. Allerdings haben nur starke Veränderungen der Gewichte Einfluss auf den Selektionserfolg.

Pärna et al. (2003) weisen darauf hin, dass es schwierig ist, die wirtschaftlichen Gewichte präzise abzuleiten, da zukünftige Preisannahmen getroffen werden müssen und diese schwer einschätzbar sind. Zudem zeigt sich der Wandel von einem Quotensystem zu einem Nicht-Quotensystem als schwierig einschätzbar. Unter den gegebenen Bedingungen in Estland zeigt sich, dass ein Quotenszenario großen Einfluss auf die wirtschaftlichen Gewichte der Milchleistung und der Nutzungsdauer hat. Auf die wirtschaftlichen Gewichte der Merkmale Verzögerungszeit bei Färsen, Zwischenkalbezeit und Erstkalbealter zeigt dies keine Auswirkungen. Die Merkmalskomplexe Milchleistung und funktionale Merkmale haben eine relative Gewichtung von 79:21 zueinander. Unter Quotenbedingungen hat das Merkmal Milchträger das höchste wirtschaftliche Gewicht, gefolgt von der Eiweißmenge (Tab. 2.7). 
Tab. 2.7 Relative wirtschaftliche Gewichte (\%) der Holsteinpopulation in Estland unter Milchquotenbeschränkungen (Pärna et al., 2003)

\begin{tabular}{lccc}
\hline & Einheit & $\begin{array}{c}\text { Genetische Standard- } \\
\text { abweichung }\end{array}$ & $\begin{array}{c}\text { Relative Gewichtung } \\
\text { im Selektionsindex }\end{array}$ \\
\hline Milchträger & $\mathrm{kg}$ & 365 & 40 \\
Fettmenge & $\mathrm{kg}$ & 12,6 & -7 \\
Eiweißmenge & $\mathrm{kg}$ & 10,6 & 32 \\
Verzögerungszeit & Tage & 10 & -9 \\
Zwischenkalbezeit & Tage & 10 & 0 \\
Nutzungsdauer & Tage & 180 & 12 \\
\hline
\end{tabular}

Pärna et al. (2003) schlussfolgern, dass die Merkmale Milchträger, Fett- und Eiweißmenge sowie die Merkmale Verzögerungszeit und Nutzungsdauer in das Zuchtziel und somit auch in den Selektionsindex einbezogen werden müssen (Tab. 2.7).

Die Auswirkungen verschiedener Preissysteme in Italien werden von Pieters et al. (1997) untersucht. Aufgrund differenzierter Produktionsrichtungen in italienischen Molkereien gibt es unterschiedliche Auszahlungssysteme für die Milchproduzenten. In allen Preissystemen wird das wirtschaftliche Gewicht für den Milchträger negativ, wenn die Milchliefermenge begrenzt wird, der höchste Gewinn bei einer Milchleistungssteigerung kann erreicht werden, wenn die Milchquotenbeschränkungen mit Referenzfettgehalt wegfallen. Allerdings haben Veränderungen im Fettauszahlungspreis die geringsten Auswirkungen auf den Gewinn im Betrieb. Fallen die Quotenbeschränkungen komplett weg, beeinflusst eine Veränderung des Auszahlungspreises für den Milchträger das relative wirtschaftliche Gewicht des Merkmals Milchträger, die relativen Gewichte der Fett- und Eiweißmenge hingegen reagieren nicht. Es wird deutlich, dass die unterstellten Szenarien mit und ohne Quotenbedingungen einen hohen Einfluss auf die Höhe der absoluten und relativen wirtschaftlichen Gewichte haben. Des weiteren untersuchen Pieters et al. (1997) den Einfluss der wirtschaftlichen Gewichte auf den Selektionserfolg. Es zeigt sich, dass ein Verlust des Zuchtfortschritts dann eintritt, wenn sich die wirtschaftlichen Gewichte auf den Zeitpunkt beziehen, an dem die Tiere selektiert werden, und nicht auf jenen, an dem der genetische Fortschritt eintreten soll. Daraus folgt, dass die unterstellten Szenarien sich auf die zukünftigen Bedingungen beziehen müssen, da es ansonsten bei Selektion nach dem Gesamtzuchtwert zu einem Verlust an Zuchtfortschritt kommt. 
Für die sächsische Rinderzucht leiten Wünsch (1999) sowie Wünsch und Bergfeld (2001) wirtschaftliche Gewichte ab. Es zeigt sich, dass es durch die Einführung einer Milchquote zum Absinken der wirtschaftlichen Gewichte der Milchleistungsmerkmale kommt. Das Verhältnis der Grenznutzen für die Fett- und Eiweißmenge wird dabei stark durch das Verhältnis der Preise für die Komponenten Milchträger, Fett- und Eiweißmenge beeinflusst. Erhöht sich die Bezahlung der Eiweißmenge im Vergleich zur Fettmenge, so steigt auch das wirtschaftliche Gewicht dieses Merkmals im Vergleich zur Fettmenge an. Bei der Ableitung der wirtschaftlichen Gewichte für die funktionalen Merkmale zeigt sich, dass diese relativ unabhängig vom Bezahlungssystem und den Szenarien für die Milchleistungsmerkmale sind. Dabei liegt das wirtschaftliche Gewicht für die Nutzungsdauer als einziges funktionales Merkmal auf einer vergleichbaren Höhe mit denen der Milchleistungsmerkmale. Des Weiteren werden Grenznutzen für die Merkmale Persistenz und Melkbarkeit abgeleitet. Der Grenznutzen der Persistenz wird überwiegend von der Futtereinsparung bei Verbesserung des Merkmals beeinflusst, während durch den Effekt der Arbeitseinsparung der Grenznutzen der Melkbarkeit bestimmt wird. Im Gegensatz zu anderen Arbeiten berechnen Wünsch und Bergfeld (2001) ein relatives wirtschaftliches Gewicht für die Zellzahl von nahezu 10\%. Im Gesamtüberblick zeigt sich, dass durch die Unterstellung einer Milchquote die relative Bedeutung der funktionalen Merkmale gegenüber den Milchleistungsmerkmalen ansteigt, da die Gewinnsteigerung im Betrieb durch die Milchquote eingeschränkt wird.

Kaltenecker (1999) stellt fest, dass sich in seinen Untersuchungen die wirtschaftlichen Gewichte zukünftig zugunsten von Milch- und Eiweißmenge verändert, die wirtschaftliche Bedeutung der Fettmenge und Fleischleistung hingegen sinkt. Dabei liegt die Gewichtung von der Fett- zur Eiweißmenge bei 1:5 und vergrößert sich nach Kaltenecker (1999) noch. Zusätzlich kommt es zu einem Vergleich zwischen Milchleistungs- und Zweinutzungsrassen. Es zeigt sich, dass sich in der Wirtschaftlichkeit eindeutig die Milchviehrasse durchsetzt, besonders wenn es weiterhin bei Milchquotenbeschränkungen und steigenden Quotenkosten bleibt.

Relative wirtschaftliche Gewichte für die Nutzungsdauer werden von Jagannatha et al. (1998) berechnet. Die wichtigen Auswirkungen, die eine verbesserte Nutzungsdauer auf den Gewinn im Betrieb hat, sind geringere Remontierungskosten auf der einen Seite und eine erhöhte Anzahl Kühe in den höheren Laktationsklassen auf der anderen Seite. Dies kann unter anderem auch zu reduzierten Merzungskosten und verringerten Kosten für Gesunderhaltung der Herde führen. Den geringsten Gewinn erreicht ein Betrieb, wenn die Preise für Milch und Schlachtkühe niedrig sind und die Preise für Futter hoch. Im umgekehrten Fall, wenn hohe Auszahlungspreise für Milch und Schlachtkühe und niedrige Futterkosten vorherrschen, wird im Betrieb ein hoher 
Gewinn realisiert. Jagannatha et al. (1998) schlussfolgern, dass eine verbesserte Nutzungsdauer eine hohe wirtschaftliche Bedeutung für den Betrieb hat und diese somit stetig erhöht werden sollte.

Bekman und van Arendonk (1993) zeigen, dass die wirtschaftlichen Gewichte der Milchleistungsmerkmale unter Milchquotenbedingungen geringer sind als im Szenario ohne Quote. Die Preise für Futtermittel, Fett- und Eiweißmenge beeinflussen die wirtschaftlichen Gewichte für die Milchleistungsmerkmale. Ebenso sind die wirtschaftlichen Gewichte für das Geburtsgewicht beeinflusst von den Kälberpreisen. Zusätzlich stellen Bekman und van Arendonk (1993) fest, dass in einem optimalen Produktionssystem die weiblichen Kälber ausschließlich zur Milchproduktion verwendet und die männlichen Kälber nur zur Mast genutzt werden sollten.

Anhand von französischen Milchviehherden berechnet Boichard (1990) wirtschaftliche Gewichte für die Konzeptionsrate. Nach der Milchleistung erweist sich dieses Merkmal als wichtigstes Merkmal in der Untersuchung. Der Milchpreis, die Nutzungsdauer der Herde und der Kälberpreis haben keinen signifikanten Einfluss auf das wirtschaftliche Gewicht der Fruchtbarkeit. Ebenso zeigen Mulder und Jansen (2001), dass die wirtschaftlichen Gewichte für die Milchleistungsmerkmale und die Nutzungsdauer wesentlich höher sind als für die Exterieurmerkmale. Weiterhin wird deutlich, dass Milchquotenbedingungen die wirtschaftlichen Gewichte der Fett- und Eiweißmenge beeinflussen.

Sölkner et al. (2000) konzipieren einen Gesamtzuchtwert für Zweinutzungsrinder und stellen die Auswirkungen eines veränderten Gesamtzuchtwertes auf den Zuchtfortschritt in der Population dar. Dabei zeigt sich, dass der höchste monetäre Zuchtfortschritt durch die Milchleistungsmerkmale realisiert wird. Eine Berücksichtigung der Fleischleistungsmerkmale erhöht den monetären Zuchtfortschritt nicht entscheidend, allerdings ist es trotz allem nötig, diese Merkmale in den Gesamtzuchtwert einzubinden. Es zeigt sich, dass eine Kombination der drei Merkmalskomplexe Milch, Fleisch und funktionale Merkmale den größten Nutzen im Zuchtfortschritt bewirkt. Des Weiteren wird die Fragestellung untersucht, ob die Exterieurmerkmale als unabhängiges Selektionskriterium betrachtet werden sollten. Der Landwirt würde aus subjektiver Sicht den Merkmalskomplex Exterieur zu 15\% im Gesamtzuchtwert gewichten. Dies ist zwar mit einem wirtschaftlichen Gewicht für diesen Komplex nicht vergleichbar, aber es zeigt die Bedeutung auf, die ein Züchter diesem Merkmal zugesteht, sei es aufgrund der Verbindung zu den übrigen funktionalen Merkmalen oder als Argument für ein gutes Aussehen der Kuh. Allerdings zeigt sich, dass mit zunehmender Gewichtung des Exterieurs als eigenständiger Komplex im Gesamtzuchtwert der monetäre Erfolg im Betrieb zurückgeht. Daher sollte eine Berücksichtigung nochmals überdacht werden. 


\subsection{Indexselektion und Gesamtzuchtwert}

In der Milchrinderzucht wird eine Gesamtindex gefordert, der sämtliche züchterisch bedeutsamen Merkmale für eine Gesamtrangierung, entsprechend ihrer wirtschaftlichen Bedeutung und ihrer genetischen Beziehungen, kombiniert (VIT, 2006). Das optimale Verfahren der Gesamtrangierung ist eine multivariate Zuchtwertschätzung bzw. ein Index, der die korrigierten Leistungsabweichungen als Informationsmerkmale verwendet. Da in der Zuchtwertschätzung in der Regel sehr komplexe und unterschiedliche Schätzsysteme zur Anwendung kommen, wird eine multivariate Schätzung derzeit nicht durchgeführt. Aus diesem Grund werden die Zuchtwerte zunächst univariat geschätzt und anschließend mittels Indexverfahren zusammengefasst.

Selektionsentscheidungen in der Tierzucht werden häufig nicht aufgrund eines einzelnen Merkmals getroffen. Es werden in der Regel mehrere Informationsquellen miteinander kombiniert. Berücksichtigt man im Zuchtziel mehrere Merkmale gleichzeitig, ist es nach Hazel und Lush (1942) die effizienteste Methode, mit der Indexselektion zu arbeiten. Je mehr Merkmale im Gesamtzuchtwert einbezogen werden, desto größer ist die Überlegenheit der Indexselektion gegenüber anderen Selektionsmethoden. Als Index wird im Allgemeinen eine Kombination mehrerer Zahlenwerte bezeichnet (Miesenberger et al., 1996). Die Indexselektion baut auf der Idee eines Gesamtzuchtwertes auf. In diesem werden alle wirtschaftlich wichtigen Merkmale berücksichtigt. Nach der Rangierung der Tiere anhand des Indexes werden Selektionsentscheidungen getroffen.

Grundsätzlich wird bei der Zuchtwertschätzung mit dem Selektionsindex das Prinzip genutzt, eine lineare Kombination der Informationsquellen zu suchen, bei der die Korrelation zwischen wahrem Zuchtwert ( $\boldsymbol{T})$ und dem geschätzten Zuchtwert (I) maximiert wird (Schüler et al., 2001). Mit Hilfe des Gesamtzuchtwertes sollen die Tiere mit dem besten Genotyp als Eltern für die nächste Generation ausgewählt werden (Miesenberger et al., 1996). Es kann zwischen dem wahren und dem geschätzten Gesamtzuchtwert unterschieden werden.

Der wahre Gesamtzuchtwert $(\boldsymbol{T})$ stellt eine gewichtete Kombination von Informationen dar und berechnet sich aus einer Zusammensetzung der Teilzuchtwerte der einzelnen Merkmale $\left(\boldsymbol{u}_{\boldsymbol{i}}\right)$ und des relativen ökonomischen Wertes des Merkmals $\left(\boldsymbol{a}_{\boldsymbol{i}}\right)$.

$$
T=a_{1} u_{1}+a_{2} u_{2}+\ldots+a_{m} u_{m}
$$


Der Gesamtzuchtwert stellt eine lineare Kombination der wahren Zuchtwerte dar. Da der wahre Zuchtwert eines Tieres nicht bekannt ist, wird versucht, diesen mit Hilfe des Selektionsindexes möglichst genau zu schätzen (Hazel, 1943). Die Ableitung des Gesamtzuchtwertes basiert auf der Selektionsindextheorie (Hazel, 1943). Die Selektion der Population nach einem Gesamtzuchtwert führt zum maximalen monetären Zuchtfortschritt im Sinne des Zuchtziels. Der Selektionsindex ist im statistischen Sinne eine multiple lineare Regression.

$$
I=b_{1} x_{1}+b_{2} x_{2}+\ldots+b_{n} x_{n}
$$

Der Selektionsindex $\boldsymbol{I}$ wird als Schätzwert für den Gesamtzuchtwert $(\boldsymbol{T})$ bezeichnet und stellt die gewichtete Summe $\left(\boldsymbol{b}_{\boldsymbol{i}}\right)$ der umweltkorrigierten Beobachtungswerte $\left(\boldsymbol{x}_{\boldsymbol{i}}\right)$ dar. Um die Gewichtungsfaktoren, d. h. die b-Werte oder Indexgewichte (b), abzuleiten, muss die Indexnormalgleichung aufgestellt werden.

$$
P \cdot b=G \cdot a
$$

$\boldsymbol{P}$ bezeichnet die phänotypische Varianz-Kovarianzmatrix zwischen den Informations- bzw. Indexmerkmalen, $\boldsymbol{G}$ die genetische Varianz-Kovarianzmatrix zwischen den Informationsmerkmalen und den Ziel- bzw. Zuchtwertmerkmalen, $\boldsymbol{a}$ den Vektor der relativen wirtschaftlichen Gewichte der Merkmale und $\boldsymbol{b}$ die Gewichtungsfaktoren im Index, die es zu berechnen gilt. Durch die Umformung der Indexnormalgleichung werden die Gewichtungsfaktoren abgeleitet.

$$
b=P^{-1} \cdot G \cdot a
$$

Der Gesamtzuchtwert wird aus den Teilzuchtwerten für die einzelnen Merkmale unter Berücksichtigung der wirtschaftlichen Gewichte, der Genauigkeit der geschätzten Zuchtwerte und der genetischen Beziehungen zwischen den Merkmalen berechnet (ZuchtData, 2005b; VIT, 2006). Der geschätzte Gesamtzuchtwert ist das beste Hilfsmittel zur Rangierung der Tiere bei komplexen Zuchtzielen (Miesenberger et al., 1996). Allerdings können und sollen der Gesamtzuchtwert und die Zuchtwertschätzung nur Entscheidungshilfen für den Züchter sein, um dem Zuchtziel näher zu kommen. 
Die Varianz-Kovarianzmatrix zwischen den Indexmerkmalen $(\boldsymbol{P})$ und die VarianzKovarianzmatrix zwischen den Zuchtziel- und Indexmerkmalen $(\boldsymbol{G})$ berechnet sich nach den Vorgaben von Reinhardt (1998).

$$
\begin{gathered}
P=\left[\begin{array}{cc}
r_{T I / u 1}^{2} \sigma^{2}\left(g_{1}\right) & r_{T I / u 1}^{2} r_{T I / u 2}^{2} \sigma^{2}\left(g_{12}\right) \\
r_{T I / u 1}^{2} r_{T I / u 2}^{2} \sigma^{2}\left(g_{12}\right) & r_{T I / u 2}^{2} \sigma^{2}\left(g_{2}\right)
\end{array}\right] \\
G=\left[\begin{array}{ll}
r_{T I / u 1}^{2} \sigma^{2}\left(g_{1}\right) & r_{T I / u 2}^{2} \sigma\left(g_{12}\right) \\
r_{T I / u 1}^{2} \sigma\left(g_{12}\right) & r_{T I / u 2}^{2} \sigma^{2}\left(g_{2}\right)
\end{array}\right]
\end{gathered}
$$

Dabei stellt $r^{2}$ die Sicherheit des geschätzten Index dar, $T$ ist der wahre und $I$ der geschätzte Zuchtwert eines Tieres, während $g$ das in der Matrix berücksichtigte Merkmal darstellt. Auf der Diagonale der Matrix stehen jeweils die Varianzen der Merkmale und auf der Off-Diagonale die Kovarianzen der Merkmale.

Die Sicherheit des geschätzten Index berechnet sich wie folgt:

$$
r^{2}=\sigma^{2}(I) / \sigma^{2}(T)
$$

Anhand der Indexgewichte werden die Teilzuchtwerte innerhalb des Gesamtzuchtwertes gewichtet.

\subsection{Wirtschaftliche Gewichte in der Zuchtwertschätzung}

Im Folgenden sind die relativen wirtschaftlichen Gewichte der Rassen Fleckvieh (Tab. 2.8) und Braunvieh (Tab. 2.9) aufgeführt (Gredler, 2004). Die Zuchtwertschätzung der beiden Rassen findet länderübergreifend in Deutschland und Österreich statt.

Der Gesamtzuchtwert „Fleckvieh 2000“ beschreibt die Gewichtung der Merkmalskomplexe, mit der ab dem Jahr 2000 in der Zuchtwertschätzung gearbeitet wurde, „Fleckvieh 2002“ beschreibt die Zusammensetzung des Gesamtzuchtwertes aus dem Jahr 2002 (Tab. 2.8). 
Tab. 2.8 Relative Gewichtung der Merkmalsgruppen Milch, Fleisch, Fitness und Melkbarkeit (MBK) im Gesamtzuchtwert „Fleckvieh 2000“ und „Fleckvieh 2002 “ (nach Gredler, 2004)

\begin{tabular}{|c|c|c|c|c|c|c|}
\hline \multirow[t]{2}{*}{ Merkmal } & & \multirow{2}{*}{ Einheit } & \multicolumn{2}{|c|}{ „Fleckvieh 2000“ } & \multicolumn{2}{|c|}{ „Fleckvieh 2002“ } \\
\hline & & & $\%$ & $\%$ & $\%$ & $\%$ \\
\hline \multirow[t]{2}{*}{ Milch } & Fkg & $\mathrm{kg}$ & 17,9 & 36,8 & 9,9 & 39,5 \\
\hline & Ekg & $\mathrm{kg}$ & 18,9 & & 29,6 & \\
\hline \multirow[t]{5}{*}{ Fleisch } & NTZ & g & & 18,3 & 9,9 & 16,5 \\
\hline & FLA & $\%$ & & & 3,3 & \\
\hline & HKL & Klasse & 2,9 & & 3,3 & \\
\hline & $\mathrm{TZ}$ & $\mathrm{g}$ & 7,7 & & & \\
\hline & AUS & $\%$ & 7,7 & & & \\
\hline \multirow[t]{9}{*}{ Fitness } & ND & Tag & 14,9 & 44,9 & 13,3 & 40,1 \\
\hline & PERS & $\mathrm{s}_{\mathrm{A}}$ & 2,0 & & 1,8 & \\
\hline & FRUp & $\%$ & 5,0 & & 4,5 & \\
\hline & FRUm & $\%$ & 5,0 & & 4,5 & \\
\hline & KVLp & Klasse & 1,2 & & 1,0 & \\
\hline & KVLm & Klasse & 1,2 & & 1,0 & \\
\hline & TOTр & $\%$ & 2,8 & & 2,5 & \\
\hline & TOTm & $\%$ & 2,8 & & 2,5 & \\
\hline & $\mathrm{ZZ}$ & $\mathrm{s}_{\mathrm{A}}$ & 10,0 & & 9,0 & \\
\hline MBK & DMG & $\mathrm{s}_{\mathrm{A}}$ & & & 3,9 & 3,9 \\
\hline
\end{tabular}

Bei Fleckvieh hat die Gewichtung der Milchleistungsmerkmale zugenommen (Tab. 2.8), während die Gewichtung der Fleischleistungsmerkmale zurückgegangen ist. Zudem ist ein gewisser Anteil der Fitnessmerkmale auf die Melkbarkeit übertragen worden. Im Gesamtzuchtwert „Fleckvieh 2002“ weist die Eiweißmenge das höchste relative wirtschaftliche Gewicht auf, gefolgt von der Nutzungsdauer, der Fettmenge und der Zellzahl. Alle anderen Merkmale haben ein wirtschaftliches Gewicht von unter $10 €$ je genetischer Standardabweichung. 
In Tab. 2.9 ist die Gewichtung des Gesamtzuchtwertes bei Braunvieh aus dem Jahr 2002 und 2004 aufgeführt (Gredler, 2004).

Tab. 2.9 Relative Gewichtung der Merkmalsgruppen Milch, Fleisch, Fitness und Melkbarkeit (MBK) im Gesamtzuchtwert bei „Braunvieh 2002“ und „Braunvieh 2004“ (nach Gredler, 2004)

\begin{tabular}{|c|c|c|c|c|c|c|}
\hline \multirow[t]{2}{*}{ Merkmal } & & \multirow{2}{*}{ Einheit } & \multicolumn{2}{|c|}{ „Braunvieh 2002““ } & \multicolumn{2}{|c|}{ „Braunvieh 2004“ } \\
\hline & & & $\%$ & $\%$ & $\%$ & $\%$ \\
\hline \multirow[t]{3}{*}{ Milch } & Fkg & $\mathrm{kg}$ & 10,8 & 50,3 & 10,2 & 47,7 \\
\hline & Ekg & $\mathrm{kg}$ & 34,5 & & 32,8 & \\
\hline & Epro & $\%$ & 5,0 & & 4,7 & \\
\hline \multirow[t]{5}{*}{ Fleisch } & NTZ & $\mathrm{g}$ & & & 3,0 & 5,0 \\
\hline & FLA & $\%$ & & & 1,0 & \\
\hline & HKL & Klasse & & & 1,0 & \\
\hline & $\mathrm{TZ}$ & $\mathrm{g}$ & & & & \\
\hline & AUS & $\%$ & & & & \\
\hline \multirow[t]{9}{*}{ Fitness } & ND & Tag & 17,4 & 45,7 & 16,5 & 43,5 \\
\hline & PERS & $\mathrm{s}_{\mathrm{A}}$ & 2,8 & & 2,7 & \\
\hline & FRUp & $\%$ & 5,2 & & 4,9 & \\
\hline & FRUm & $\%$ & 5,2 & & 4,9 & \\
\hline & KVLp & Klasse & 0,7 & & 0,7 & \\
\hline & KVLm & Klasse & 0,7 & & 0,7 & \\
\hline & TOTp & $\%$ & 2,2 & & 2,1 & \\
\hline & TOTm & $\%$ & 2,2 & & 2,1 & \\
\hline & $\mathrm{ZZ}$ & $\mathrm{s}_{\mathrm{A}}$ & 9,3 & & 8,9 & \\
\hline MBK & DMG & $\mathrm{s}_{\mathrm{A}}$ & 4,0 & 4,0 & 3,8 & 3,8 \\
\hline
\end{tabular}

Wie in Tab. 2.9 zu sehen ist, wurde beim Gesamtzuchtwert „Braunvieh 2004“ zusätzlich zu „Braunvieh 2002“ die Fleischleistung integriert (Gredler, 2004). Das höchste 
relative wirtschaftliche Gewicht hat auch beim Braunvieh die Eiweißmenge, gefolgt von der Nutzungsdauer, der Fettmenge und der Zellzahl. Alle anderen Merkmale haben ein wirtschaftliches Gewicht von unter $10 €$ je genetischer Standardabweichung. Zusätzlich werden wirtschaftliche Gewichte für die Fleischleistungsmerkmale Tägliche Zunahme (TZ) und Ausschlachtung (AUS) abgeleitet, die aber lediglich als Hilfsmerkmale in die Berechnung eingehen. Durch die Implementierung der Fleischleistung in den Gesamtzuchtwert haben die Milchleistungsmerkmale ebenso wie die Fitnessmerkmale an Gewicht verloren.

\subsubsection{Gesamtzuchtwert Holstein}

Im Jahr 1997 wurde erstmals ein Gesamtzuchtwert (RZG) vom Deutschen Holstein Verband (DHV e.V.) eingeführt, der alle züchterisch wichtigen Merkmale entsprechend ihrer Gewichtung im Zuchtziel kombiniert (VIT, 2006). Der Gesamtzuchtwert wurde im Jahr 2002 verändert und es wurde den neuen wirtschaftlichen und politischen Rahmenbedingungen sowie Neuerungen in der Zuchtwertschätzung Berücksichtigung geschenkt. Der Gesamtzuchtwert (RZG) der Rasse Holstein setzt sich aus fünf Teilzuchtwerten zusammen.

Die Relativzuchtwerte werden auf einer Skala mit Mittelwert 100 und einer genetischen Streuung von 12 Punkten für die aktuellen Bullenjahrgänge ausgewiesen (VIT, 2006). Alle Relativzuchtwerte werden, mit Ausnahme des RZN, berechnet und veröffentlicht, wenn für die darin enthaltenen Einzelmerkmale Zuchtwerte mit mindestens 50\% Sicherheit geschätzt sind. Der RZG wird ausgewiesen, sobald die Teilzuchtwerte RZM, RZE und RZS mit ausreichender Sicherheit von mindestens 50\% vorliegen. Die nationale Zuchtwertschätzung erfolgt für die Merkmale Milchleistung, Zellzahl, Exterieur und Nutzungsdauer dreimal jährlich. Die Zuchtwerte für die Kalbe- und Fruchtbarkeitsmerkmale werden bisher nur einmal jährlich geschätzt.

\section{Relativzuchtwert Milchleistung (RZM)}

Im RZM werden die Merkmale Fett- und Eiweißmenge berücksichtigt. Das Verhältnis von Fett- zu Eiweißmenge liegt bei 1:4 Einheiten. Die Inhaltsstoffe Fett- und Eiweißprozent fließen in den Relativzuchtwert mit ein, um dem negativen Trend der Fett- und Eiweißgehalte, der sich nach Einführung des RZM bzw. RZG eingestellt hat, entgegen zu wirken. 


\section{Relativzuchtwert Exterieur (RZE)}

In der Zuchtwertschätzung für Exterieur werden die Daten verwendet, die bei der linearen Beschreibung und Bewertung von Kühen und Nachzuchttieren erhoben werden. Für die Einzelzuchtwerte der Linearmerkmale werden Indices für die Merkmalskomplexe Milchtyp, Körper, Fundamente und Euter berechnet. Im RZE werden die Teilzuchtwerte der Merkmalskomplexe in einem Relativzuchtwert kombiniert.

\section{Relativzuchtwert Somatischer Zellgehalt (RZS)}

Die Zuchtwertschätzung des somatischen Zellgehaltes verwendet die Zellzahlergebnisse aus den Einzelkontrollen als Informationsmerkmale. Um eine Normalverteilung zu erzielen, wird der somatische Zellgehalt mithilfe einer logarithmischen Transformation zum Somatic Cell Score (SCS) umgewandelt. Im RZS wird die Milchleistungsminderung infolge einer Mastitiserkrankung nicht berücksichtigt, da dies bereits in der Zuchtwertschätzung Milchleistung mit eingeht.

\section{Relativzuchtwert Nutzungsdauer (RZN)}

Zur Beschreibung der Gesundheit und Konstitution einer Kuh dient das Merkmal funktionale Nutzungsdauer. Bei den Abgangsgründen einer Kuh ist zu unterscheiden zwischen der freiwilligen Merzung, die einer Leistungsselektion durch den Züchter gleichkommt, und der unfreiwilligen Merzung, bei der die Kuh aufgrund mangelnder Fitness aus dem Betrieb ausscheiden muss. Die funktionale Nutzungsdauer ist eine um die freiwillige Merzung korrigierte Nutzungsdauer, die das genetische Potential eines Tieres widerspiegelt.

Die Ergebnisse aus der direkten Zuchtwertschätzung Nutzungsdauer werden nicht veröffentlicht (VIT, 2006). Um die Genauigkeit der Zuchtwertschätzung zu erhöhen, werden die Ergebnisse mit Hilfe eines Selektionsindexes mit den Zuchtwerten korrelierter Informationsmerkmale kombiniert. Zu den Informationsmerkmalen für die Nutzungsdauer zählen die Zellzahl-, Körpertiefe-, Fundamentnote- und Eutertiefezuchtwert sowie der Zuchtwert für die maternale Totgeburtenrate. Im RZN sind die durch die Indexkombination ermittelten Zuchtwerte zusammengefasst.

\section{Relativzuchtwert Zuchtleistung (RZZ)}

Die Zuchtleistungsmerkmale sind die Merkmale Kalbeverlauf, Kälberverluste und Fruchtbarkeit (VIT, 2006). Im RZZ werden diese Merkmale als Relativzuchtwert zusammengefasst. Die Berechnung erfolgt mithilfe eines Selektionsindex, der die 
Merkmale unter Berücksichtigung ihrer wirtschaftlichen Bedeutung, der genetischen Beziehungen und der Sicherheit der Einzelzuchtwerte miteinander kombiniert. Die Merkmale sind bereits innerhalb der Merkmalskomplexe zu Teilzuchtwerten zusammengefasst. Zukünftig wird eine Umstellung der Zuchtwerte für die weibliche Fruchtbarkeit erfolgen (Rensing, 2007). Die Zwischenkalbezeit wird als neues Merkmal mit in die Zuchtwertschätzung aufgenommen, da diese ein übergeordnetes zusammenfassendes Fruchtbarkeitsmerkmal darstellt. Zudem ist die Datengrundlage dieses Merkmals im Gegensatz zu anderen Fruchtbarkeitsmerkmalen in der Praxis sehr gut. So wird in Zukunft die Zuchtwertschätzung Fruchtbarkeit mit einem Mehrmerkmalsmodell mit Rastzeit, Non-Return-Rate und Zwischenkalbezeit durchgeführt.

Miglior et al. (2005) stellen die Gesamtzuchtwerte bei Holstein im internationalen Vergleich dar (Abb. 2.1).

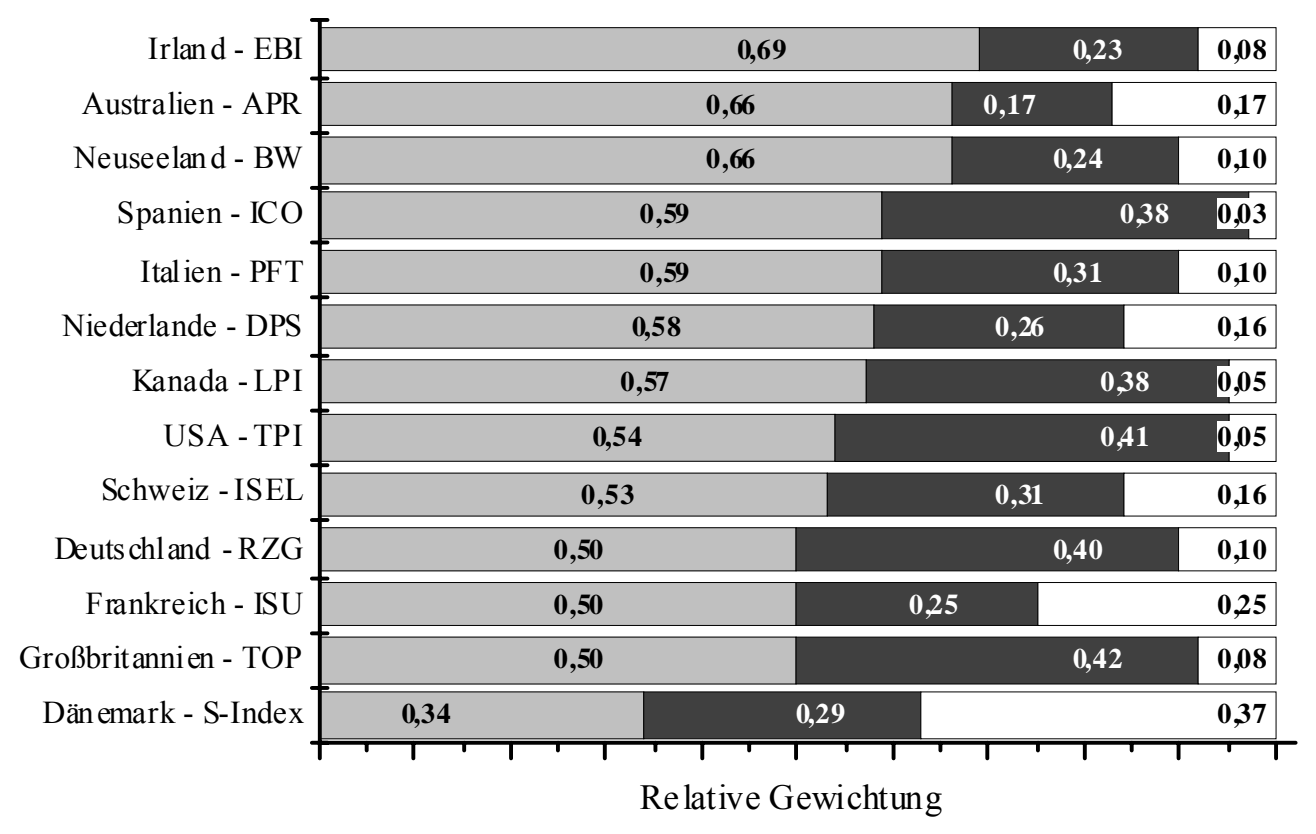

Produktion

Nutzun gsdauer Gesun dheit und Reproduktion

Abb. 2.1 Relative Gewichtung der Merkmalskomplexe Milchleistung und Lebensdauer sowie Gesundheit und Reproduktion in den Gesamtzuchtwerten einzelner Länder im August 2003 (nach Miglior et al., 2005) 
Es zeigt sich, dass in Deutschland bei der Rasse Holstein im Vergleich zu anderen Ländern schon wenig Gewichtung auf den Milchleistungsmerkmalen liegt, die Komplexe, die mit der Lebensdauer verknüpft sind, hingegen schon beträchtlichen Anteil erreicht haben (Abb. 2.1). Im Wandel der letzten Jahre ist es in anderen Ländern jedoch auch zu einer Abnahme in der Gewichtung der Milchleistungsmerkmale gekommen.

\subsubsection{Gesamtzuchtwert Fleckvieh und Braunvieh}

Beim Fleckvieh und Braunvieh setzt sich der ökonomische Gesamtzuchtwert (GZW) aus den Merkmalsbereichen Milchwert, Fleischwert und Fitnesswert zusammen.

\section{Milchwert $(M W)$}

In den Milchwert bei Fleckvieh gehen die Merkmale Fettmenge und Eiweißmenge mit ein. Diese sind im Verhältnis 1:4 zueinander gewichtet (ZuchtData, 2005b). Bei Braunvieh sind neben der Fett- und Eiweißmenge noch die Eiweißprozente mit in den Milchwert aufgenommen.

\section{Fleischwert (FLW)}

Der Fleischwert bei Fleckvieh setzt sich aus den Merkmalen Nettozunahme, Ausschlachtungsprozente und Handelklasse zusammen. Dabei hat die Nettozunahme den größten Anteil an dem Merkmalskomplex. Bei Braunvieh geht anstelle der Ausschlachtungsprozente die Fleischleistung mit ein. Für die süddeutschen Zweinutzungsrassen erfolgt die Zuchtwertschätzung für Fleischleistung seit 1996 mit einem Mehrmerkmalstiermodell (Schild und Niebel, 1998). Dabei werden alle Leistungsmerkmale der offiziellen Fleischleistungsprüfung berücksichtigt. 


\section{Fitnesswert $(F W)$}

Der Fitnesswert wird sowohl bei Fleckvieh als auch bei Braunvieh aus den Merkmalen Nutzungsdauer, Persistenz, Fruchtbarkeit, Kalbeverlauf, Totgeburtenrate und Zellzahl kombiniert. Die Melkbarkeit ist ein Einzelmerkmal, was diesem Komplex jedoch am besten zugeordnet werden kann. Grundsätzlich ist bei der Gewichtung der einzelnen Merkmale in einem umfangreichen Index zu berücksichtigen, dass es sich um eine Kompromisslösung handelt, die das Ziel hat, den bestmöglichen Zuchtfortschritt in allen Merkmalskomplexen zu realisieren. Die Berechnung eines Gesamtzuchtwertes ist aber nach wie vor sinnvoll, da ansonsten die Vielzahl an Einzelinformationen ungewichtet nebeneinander stehen würde. 


\section{$3 \quad$ Berechnung der wirtschaftlichen Gewichte}

Für die Berechnung der wirtschaftlichen Gewichte wurde ein von Amer et al. (1994) entwickeltes Computerprogramm verwendet. Das in Fortran 77 geschriebene Programm wurde zunächst zur Optimierung von Managemententscheidungen entwickelt. Miesenberger (1997) erweiterte und adaptierte das Programm an die österreichischen Bedingungen und simuliert einen Milchviehbetrieb mit Jungviehaufzucht und Bullenmast, so dass es zur Berechnung von Grenznutzen und wirtschaftlichen Gewichten genutzt werden kann.

Miesenberger (1997) leitet Grenznutzen bzw. wirtschaftliche Gewichte für die Merkmale Milchträger (fett- und eiweißfreie Milch), Fett- und Eiweißmenge, Nutzungsdauer, tägliche Zunahme, Ausschlachtungsprozente und Handelsklasse (EUROP), Nutzungsdauer, Fruchtbarkeit, Kalbeverlauf und Totgeburtenrate. Im Modell wurden Erweiterungen um die Merkmale Persistenz, SCS (Somatic Cell Score), Melkbarkeit und Exterieur vorgenommen. Die Parameter für diese Merkmale wurden sowohl bei der Berechnung der Kosten und Erlöse im Betrieb einbezogen als auch in der Aufstellung der Herdenparameter berücksichtigt. Das Merkmal tägliche Zunahme wurde auf das in der Zuchtwertschätzung verwendete Merkmal Nettozunahme korrigiert.

\subsection{Beschreibung des Modells}

Bei dem von Miesenberger (1997) verwendeten Herdenmodell handelt es sich um ein deterministisches Modell. Eine Milchviehherde wird mit den Teilbereichen Milchviehhaltung, Kälberaufzucht und Bullenmast in einem statischen Zustand abgebildet. Zur Ableitung der wirtschaftlichen Gewichte werden die Parameter in den berücksichtigten Merkmalen variiert und aus der daraus resultierenden Veränderung im Durchschnittsgewinn wird mittels Differenzenrechnung das wirtschaftliche Gewicht des Merkmals bestimmt. Eine wesentliche Einschränkung bei der Ableitung wirtschaftlicher Gewichte ist der Umstand, dass die Berechnung im Modell anhand einer konkreten Ausgangssituation vorgenommen wird. In der Realität besteht jedoch eine große Variabilität innerhalb der einzelnen Betriebsparameter, die bei der Berechnung anhand eines Durchschnittsbetriebs nicht berücksichtigt werden kann. 
Aufgrund dessen wird eine Weiterentwicklung der Methode vorgestellt, die es ermöglicht, die Realität direkter in das Modell mit einzubeziehen.

\subsubsection{Mittelwerte und Varianzen der wirtschaftlichen Gewichte}

Für verschiedene Variablen $\mathrm{x}, \mathrm{y}, \ldots, \mathrm{z}$ wird jeweils eine Verteilung (Dichtefunktion) $\mathrm{f}(\mathrm{x}), \mathrm{f}(\mathrm{y}), \ldots, \mathrm{f}(\mathrm{z})$ vorgegeben. Bei den Variablen kann es sich z. B. um die mittlere Milchleistung, die Herdengröße oder den Milchpreis handeln. Diese Dichtefunktionen, die verschiedene Formen annehmen können, sollten die erwartete Verteilung der jeweiligen Variablen am Ende des Planungshorizontes widerspiegeln, wobei die Varianz sowohl die biologische Variabilität (z. B. Milchmenge) als auch die Unsicherheit über zukünftige Produktions- und Marktbedingungen (z. B. Milchauszahlungspreis) abbilden kann. Es wird unterstellt, dass die Variablen unabhängig verteilt sind. Das Amer-Miesenberger-Programm (Amer et al., 1994; Miesenberger, 1997) wurde dahingehend weiterentwickelt, dass in einem Rechenschritt die wirtschaftlichen Gewichte für eine Vielzahl von Parameterkombinationen berechnet werden können. Dies kann sowohl über ein mehrdimensionales Gitter als auch für zufällig generierte Parameterkombinationen geschehen. Die Ergebnisse werden anschließend gewichtet zusammengefasst. Dies resultiert in einer Verteilung der wirtschaftlichen Gewichte, die durch Mittelwert und Streuung charakterisiert werden kann.

Die Vorgehensweise soll am Beispiel der Einbeziehung zweier Variablen ( $x=$ Milchleistung und $y=$ Milchpreis) dargestellt werden:

Für eine bestimmte Realisierung der mittleren Milchmenge $x_{i}$ und des Milchpreises $y_{j}$ wird das wirtschaftliche Gewicht $w_{i j}$ ermittelt. Dieses Szenario hat die Wahrscheinlichkeitsdichte $p_{i j}=f\left(x_{i}\right) \cdot f\left(y_{j}\right)$. Aus vielen Kombinationen der beiden Parameter errechnen sich der Mittelwert (3.1) und die Varianz (3.2) des wirtschaftlichen Gewichtes.

$$
\begin{gathered}
E(w)=\frac{\sum_{i, j} p_{i j} w_{i j}}{\sum_{i, j} p_{i j}} \\
\operatorname{Var}(w)=\frac{\sum_{i, j} p_{i j}\left(w_{i j}-E(w)\right)^{2}}{\sum_{i, j} p_{i j}}
\end{gathered}
$$


In Abb. 3.1 ist die Berechnung der Mittelwerte der wirtschaftlichen Gewichte aus einer Vielzahl wirtschaftlicher Gewichte und der Häufigkeitsverteilung der Variablen dargestellt.

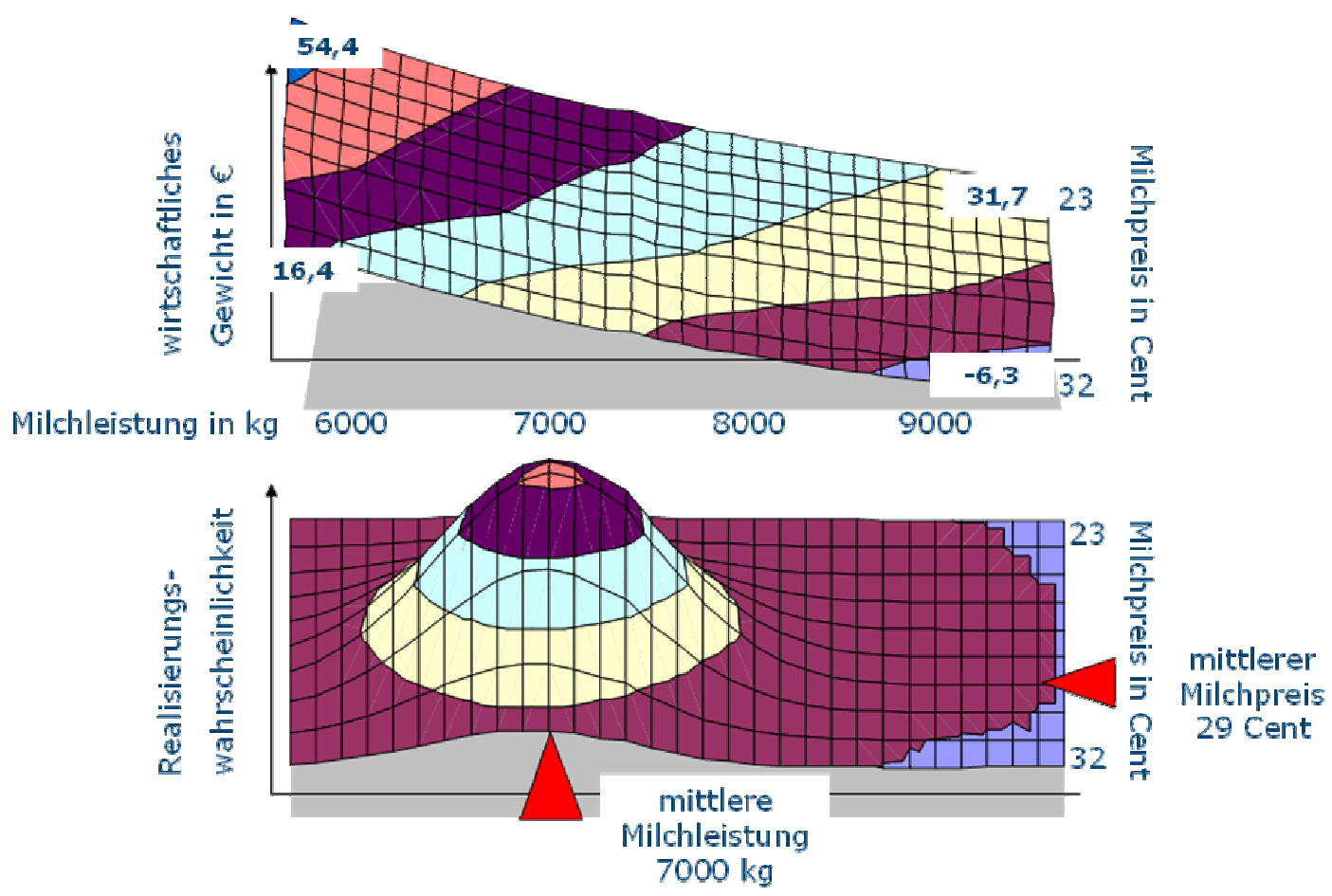

Abb.3.1 Wirtschaftliche Gewichte und Häufigkeitsverteilung am Beispiel Fettmenge bei Fleckvieh im Referenzszenario

Zunächst sind die abgeleiteten wirtschaftlichen Gewichte des Merkmals Fettmenge bei der Rasse Fleckvieh im Referenzszenario dargestellt (Abb. 3.1). Sowohl die Milchleistung in der Herde als auch der Milchpreis in der Herde werden variiert und für jegliche Kombination wird ein wirtschaftliches Gewicht berechnet. Für die Parameterkombination wird eine Häufigkeitsverteilung für die Herdenparameter festgelegt, um dadurch auf die Verteilung in der Population zurück schließen zu können. Kombiniert man die wirtschaftlichen Gewichte und die Häufigkeitsverteilung bei dem jeweiligen Merkmal miteinander, so berechnet man den Mittelwert und die Varianz des untersuchten Merkmals und kann dadurch die Unsicherheiten in der Einschätzung der zukünftigen Produktion minimieren.

Die Verwendung des Programms und des Modells wird nach Miesenberger (1997) durchgeführt. In der vorliegenden Arbeit werden ausschließlich die erweiterten Teile des Modells erläutert. Dies sind die zusätzlichen Merkmale SCS und Exterieur, für 
die jeweils ein eigenes wirtschaftliches Gewicht berechnet wird. Des Weiteren werden die in das Modell eingehenden Parameter beschrieben.

\subsubsection{Milchviehherde}

Die Milchviehherde befindet sich in einem Gleichgewichtszustand und es wird von einer über die Zeit konstanten Altersverteilung ausgegangen. Die in der Herde gemerzten Kühe werden durch die eigene Nachzucht ersetzt.

\subsubsection{Kalbezyklus und Nutzungsdauer}

Der Kalbezyklus spiegelt die Zeit wider, in der am gleichen Kuhplatz wieder eine Kalbung erfolgt. Dies kann entsprechend der Aufteilung der Herde in den einzelnen Klassen und anhand der Verweildauer in den Klassen berechnet werden. Die tatsächliche Nutzungsdauer ist als die Zeit zwischen dem Erstkalbealter und dem Alter beim Abgangszeitpunkt einer Kuh definiert. Aus diesem Grund kann diese nur für Kühe in Abgangsklassen berechnet werden. Für alle Kuhklassen wurde im Modell das gleiche Erstkalbalter unterstellt. Daher weicht die Nutzungsdauer für Kühe in den Abgangsklassen nur von der Anzahl der Laktationen mit entsprechenden Zwischenkalbezeiten und der Verweildauer in der Herde ab.

\subsubsection{Milchleistung und Körpergewicht}

Die Beschreibung der täglichen Milch-, Fett- und Eiweißleistung erfolgt über die von Wood (1967) beschriebene Exponentialfunktion zur Beschreibung der Laktationskurve (Miesenberger, 1997). Die Milch-, Fett- und Eiweißleistung $\boldsymbol{y}$ am Tag $\boldsymbol{t}$ wird durch die Parameter $\boldsymbol{a}$ für das Leistungsniveau, $\boldsymbol{b}$ für den Kurvenanstieg zu Beginn der Laktation und $c$ für den Kurvenabfall im Verlauf der Laktation bestimmt. Der Parameter für das Leistungsniveau (a) wird intern im Programm berechnet. Eine Unterscheidung findet zwischen den einzelnen Laktationskurven der ersten, zweiten und höheren Laktationen statt.

$$
y_{t}=a \cdot t^{b} \cdot e^{c \cdot t}
$$

Alle Funktionen und Parameter zur Beschreibung und Berechnung des Körpergewichtes und der Gewichtsveränderung basieren auf den Grundlagen des AmerMiesenberger-Programms (Amer et al., 1994; Miesenberger, 1997). 


\subsubsection{Grundfutteraufnahme und Bedarf in der Fütterung}

Die tägliche Futterration wird in Abhängigkeit der Milchleistung mittels Linearer Programmierung berechnet (Miesenberger, 1997). Dadurch wird sichergestellt, dass die günstigste Ration aus den zur Verfügung stehenden Futtermitteln, unter Berücksichtigung von Futteraufnahmekapazitäten und Restriktionen der Futtermittelanteile, zusammengestellt wird. Die Berechnung der maximalen Futteraufnahmekapazität für Milchkühe (kg TS je Kuh und Tag) basiert auf von Gruber et al. (2004) ermittelten Regressionsgleichungen. Die Ableitung des Energiebedarfs (MJ NEL) und des Eiweißbedarfs (g XP) je Tag erfolgt nach Vorgaben von Jeroch et al. (1999).

Im Modell muss sowohl dem Energiedefizit der Kuh als auch deren Reservehaushaltung Rechnung getragen werden. Das Energiedefizit wird aus der Differenz des Energiebedarfs und der maximalen Energieaufnahme berechnet (Miesenberger, 1997). Kommt es zu einem Energiedefizit im ersten Drittel der Laktation, wird dieses durch Körpergewichtsabbau bis zu einer maximalen Rate von $7,77 \mathrm{~g} / \mathrm{kg} \mathrm{LG}^{0,75}$ und Tag ausgeglichen. Dies ist allerdings nur bis zu einem maximalen Gewichtsverlustes von $8 \%$ des Lebendgewichtes zum Zeitpunkt der letzten Abkalbung möglich. Ist dieser Ausgleich nicht möglich, wird eine Reduzierung der täglichen Milchmenge über die Wood-Kurve realisiert, um eine ausgeglichene Energiebilanz herzustellen. Erreicht eine Kuh einen positiven Energiestatus durch alleinige Grundfutterfütterung, so ist davon auszugehen, dass dieser Überschuss zum Aufbau körpereigener Reserven verwendet wird. Diese Gewichtszunahme wird linear modelliert, damit die Kuh zum Zeitpunkt der Abkalbung ein optimales Kalbegewicht erreicht hat.

\subsubsection{Durchschnittliche Klassenergebnisse beim Milchvieh}

Zur Kalkulation der Ergebnisse werden die Berechnungen der einzelnen Klassen mit der Wahrscheinlichkeit des Auftretens eines Merkmals in einer Klasse gewichtet. Um zu den Ergebnissen des gesamten Betriebs zu gelangen, werden die Ergebnisse aus allen Teilbereichen, d. h. dem Milchviehbereich, der Jungviehaufzucht und der Bullenmast, zusammengefügt. Dies wird dann als durchschnittliches Ergebnis des Milchviehbereiches je Herdendurchschnittskuh und Kalbezyklus ausgegeben.

\subsubsection{Jungvieh}

In der Jungviehaufzucht wird nicht nach Alter- oder Abgangsklassen unterschieden. Das Verbleiben eines Kalbes im Betrieb hängt zum einen vom Geschlecht und zum anderen vom Management des Betriebes ab. Um die Ergebnisse je Herdendurch- 
schnittskuh berücksichtigen zu können, ist es notwendig, die Realisierungswahrscheinlichkeiten je Kalbezyklus zu berücksichtigen.

Die Berechnung der Realisierungswahrscheinlichkeit ist nötig, da nicht jede Kuh mit der Geburt eines weiblichen Kalbes zur Nachzucht beiträgt. Hinzu kommt noch die Wahrscheinlichkeit eines Abgangs oder Ausfalls während der Aufzucht.

\subsubsection{Herdenaufbau und Fütterung}

Für die Bestandsergänzung wird ein bestimmter Anteil lebend geborener weiblicher Kälber benötigt. Zusätzlich werden die Wahrscheinlichkeiten für Aufzuchtverluste und Unfruchtbarkeit berücksichtigt. Daraus leitet sich der Bedarf an weiblichen Kälbern je Kalbezyklus, die zur Remontierung benötigt werden, ab. Weibliche Kälber, die dem Betrieb zur Verfügung stehen, aber nicht zur Remontierung benötigt werden, werden zur Zucht verkauft.

Die Fütterung in der Kälberaufzuchtphase erfolgt nach einem Tränke- und Fütterungsplan für Frühentwöhnung (Miesenberger, 1997). Die Fütterungskosten für Jungtiere werden täglich ermittelt und über die gesamte Periode bis zur Abkalbung oder zum Abgang zusammengefasst. Ähnlich wie im Milchviehbereich wird die tägliche Optimierung der Ration mithilfe Linearer Programmierung durchgeführt.

\subsubsection{Ergebnisse der Jungviehaufzucht}

Bei der Jungviehaufzucht wird zwischen Jungtieren unterschieden die entweder für die Bestandergänzung aufgezogen werden oder die für den Zuchtviehverkauf bestimmt sind. Die Aufzuchtstrategien unterscheiden sich lediglich in der Haltungsdauer, was jedoch zu unterschiedlichen Aufzuchtkosten führt. Sämtliche Kosten, die in der Jungviehaufzucht anfallen, werden als Kosten für die Bestandsergänzung dem Milchviehbereich zugeschlagen. Die Ergebnisse je Jungtier werden bei der Berechnung der Ergebnisse je Herdendurchschnittskuh berücksichtigt.

\subsubsection{Bullenmast}

In dieser Arbeit wird Bullenmast in Fleckvieh- und Braunviehbetrieben durchgeführt. Die Entscheidung, ob ein Kalb nach der Kälberaufzuchtphase in den Bereich der Jungviehaufzucht oder in die Bullenmast gelangt, liegt neben dem Geschlecht am unterstellten Management. Alle männlichen Kälber werden zur Mast im eigenen Betrieb eingestallt. 


\subsubsection{Herdenaufbau und Fütterung}

Um die Ergebnisse je Herdendurchschnittskuh berücksichtigen zu können, wird die Realisierungswahrscheinlichkeit für die Geburt und das Überleben von männlichen und weiblichen Kälbern bestimmt (Miesenberger, 1997).

Das Lebendgewicht (3.4) und die täglichen Zunahmen (3.5) je Mastbullen werden mit der Gompertz-Funktion (Fitzhugh, 1976) berechnet.

$$
\begin{gathered}
L W_{t}=a \cdot e^{\left(-b \cdot e^{(-k \cdot t)}\right)} \\
D G_{t}=a \cdot k \cdot b \cdot e^{(-k \cdot t)} \cdot e^{\left(-b \cdot e^{(-k \cdot t)}\right)}
\end{gathered}
$$

Die Fütterungskosten je Mastbulle werden täglich errechnet und über die gesamte Periode zusammengefasst. Wie bei der Milchkuh- und Jungviehfütterung wird die tägliche Fütterungsration mittels Linearer Programmierung optimiert.

\subsubsection{Ergebnisse der Bullenmast}

Die Ergebnisse aus der Bullenmast enthalten neben den Ergebnissen aus der direkten Bullenmast auch die der Kälberfütterungsphase sowie die Kosten für Verluste. Im Modell kann die Mastdauer entweder in Abhängigkeit der täglichen Zunahme bei Erreichen eines vorgegebenen Mastendgewichtes oder über eine fixe Mastdauer bestimmt werden. Die Ergebnisse werden schließlich auf eine Herdendurchschnittskuh je Jahr bezogen.

\subsubsection{Gesamtergebnisse}

Für die Berechnung des Gesamtergebnisses je Herdendurchschnittskuh und Kalbezyklus werden die Ergebnisse des Milchviehbereiches und die Ergebnisse der Jungviehaufzucht entsprechend der Realisierungswahrscheinlichkeiten je Geburt zusammengefasst. Werden im Betrieb zusätzlich Bullen gemästet, fließen auch diese Ergebnisse in die Berechnung mit ein. Zusätzlich werden die Ergebnisse je Herdendurchschnittskuh und Jahr ausgewiesen. Dafür wird das Gesamtergebnis je Herdendurchschnittskuh und Kalbezyklus mit der durchschnittlichen Anzahl der Abkalbungen je Kuhplatz und Jahr gewichtet. 
Um Rückschlüsse auf den Betrieb ziehen zu können, werden die Ergebnisse je Betrieb und Jahr berechnet, was den Ergebnissen der Gesamtherde entspricht. Die Anzahl der Kuhplätze je Betrieb errechnet sich aus der vorgegebenen Milch-, Fettund/oder Eiweißquote des Betriebes und der Leistung des unter Quote stehenden Merkmals. Es werden die Erlöse, Kosten und Faktoransprüche je Herde ausgewiesen.

\subsection{Referenzszenario}

Um das Referenzszenario genauer charakterisieren zu können, werden im Folgenden die im Modell verwendeten Parameter sowie Ertrag- und Kostenkomponenten beschrieben. Es werden wirtschaftliche Gewichte für die Rassen Holstein, Fleckvieh und Braunvieh unter Annahme eines freien Marktes ohne Quotenbeschränkungen abgeleitet. Die Parameter zur Beschreibung der Herde und zur Abschätzung der Kosten und Erträge sind zum Großteil den Betriebszweigauswertungen der Länder Bayern (LfL, 2004a) und Schleswig-Holstein (LKV, 2004) sowie allgemeinen Bewertungen (KTBL, 2005) entnommen. Die Berechnungen basieren auf repräsentativen Daten der Population für die Leistungs-, Erlös- und Kostenstrukturen sowie auf populationstypischen biologischen und technischen Parametern. Hierzu wurde ein Datenmaterial ausgewertet, das 158 Milchviehbetriebe aus Bayern mit der Rasse Fleckvieh und 449 Betriebe aus Schleswig-Holstein mit der Rasse Holstein umfasst.

\subsubsection{Aufbau und Altersstruktur}

Die Annahmen zum Aufbau und der Altersstruktur der Herde sind in Tab. $3.1 \mathrm{zu}$ sammengefasst (LfL, 2004a; LKV, 2004) und basieren auf den Auswertungen der Bundesländer Schleswig-Holstein für die Rasse Holstein und Bayern für die Rassen Fleckvieh und Braunvieh. 
Tab. 3.1 Aufteilung der lebenden Kühe auf die einzelnen Laktationen bei den Rassen Fleckvieh und Braunvieh (LfL, 2004a) sowie Holstein (LKV, 2004)

\begin{tabular}{lccccccccc}
\hline \multirow{2}{*}{ Rasse } & \multicolumn{1}{c}{ Laktation } \\
\cline { 2 - 10 } & 1 & 2 & 3 & 4 & 5 & 6 & 7 & 8 & 9 \\
\hline Fleckvieh & 31,5 & 24,0 & 18,0 & 12,0 & 6,7 & 3,9 & 2,1 & 1,8 & \\
Holstein & 36,7 & 25,6 & 16,1 & 10,3 & 5,8 & 3,0 & 1,4 & 0,6 & 0,5 \\
Braunvieh & 27,3 & 22,7 & 16,5 & 12,7 & 8,7 & 5,4 & 3,2 & 3,3 & \\
\hline
\end{tabular}

Im Modell werden maximal neun Laktationen und drei verschiedene Abgangsgründe je Laktation berechnet, woraus sich 36 Kuhklassen ergeben. Die Unterscheidung bei den Abgangsgründen wird zwischen unfreiwilliger Merzung, freiwilliger Merzung und Merzung wegen Unfruchtbarkeit gemacht. Am Ende der neunten Laktation scheiden alle Kühe aufgrund unfreiwilliger Merzung aus. Die Wahrscheinlichkeit wegen Unfruchtbarkeit aus der Herde auszuscheiden ergab sich aus den vorgegebenen Besamungserfolgen und der Anzahl der maximal möglichen Besamungsversuche. Im Modell wird für denselben Abgangsgrund auch der gleiche Abgangszeitpunkt unterstellt. Ebenso wird bei der Rast- und Verzögerungszeit nicht zwischen den Laktationen unterschieden. Es ergeben sich aufgrund von unterschiedlich angenommenen Konzeptionsraten verschiedenen Zwischenkalbezeiten in den einzelnen Laktationsklassen.

\subsubsection{Milchleistung und Milcherlös}

Des Weiteren werden die Milchleistung und Milchinhaltsstoffe der ersten drei Laktationen beschrieben, aus denen sich die restliche Herdenverteilung berechnet (Tab. 3.2). 
Tab. 3.2 305-Tage-Leistung für Milchmenge (kg), Fett- (\%) und Eiweißgehalt (\%) für die Rassen Fleckvieh und Braunvieh (LKV, 2005) sowie Holstein (LKV, 2004)

\begin{tabular}{cccccccccc}
\hline & \multicolumn{3}{c}{ Fleckvieh } & \multicolumn{3}{c}{ Holstein } & \multicolumn{3}{c}{ Braunvieh } \\
\cline { 2 - 9 } Laktationen & Milch & Fett & Eiweiß & Milch & Fett & Eiweiß & Milch & Fett & Eiweiß \\
\hline 1 & 7000 & 4,12 & 3,45 & 8000 & 4,10 & 3,36 & 7150 & 4,12 & 3,50 \\
2 & 7700 & 4,10 & 3,50 & 8700 & 4,13 & 3,39 & 8000 & 4,14 & 3,57 \\
3 & 8000 & 4,08 & 3,45 & 9000 & 4,14 & 3,34 & 8400 & 4,15 & 3,52 \\
\hline
\end{tabular}

Um die einzelnen Laktationsleistungen in der Herde berechnen zu können, werden Alterungsfaktoren für die einzelnen Laktationsklassen vorgegeben (Tab. 3.3).

Tab. 3.3 Alterungsfaktoren für die Berechnung des Milchleistungspotentials in den einzelnen Laktationen bei Fleckvieh und Braunvieh (LKV, 2005) sowie Holstein (LKV, 2004)

\begin{tabular}{lccccccccc}
\hline & \multicolumn{1}{c}{ Laktation } \\
\cline { 2 - 10 } Rasse & 1 & 2 & 3 & 4 & 5 & 6 & 7 & 8 & 9 \\
\hline Fleckvieh & 1,00 & 1,10 & 1,13 & 1,14 & 1,12 & 1,09 & 1,02 & 1,02 & 1,02 \\
Holstein & 1,00 & 1,14 & 1,20 & 1,20 & 1,19 & 1,17 & 1,14 & 1,09 & 1,08 \\
Braunvieh & 1,00 & 1,11 & 1,17 & 1,17 & 1,16 & 1,14 & 1,12 & 1,06 & 1,05 \\
\hline
\end{tabular}

Für die 305-Tage Standardlaktation in der ersten Laktation wurde das Milchleistungspotential vorgegeben, jenes für die höheren Laktationen wird mithilfe der Alterungsfaktoren multiplikativ ermittelt.

Der Milchpreis, der in das Modell eingeht, setzt sich aus den Komponenten Grundpreis, Fettpreis und Eiweißpreis zusammen. Der für den Holsteinbetrieb unterstellte Milchpreis ist an den aus dem Jahr 2006 ausbezahlten Milchpreis in Norddeutschland angelehnt. Es wurden 0,02€ Grundpreis, 2,20€ je kg Fettmenge und 5,00€ je kg Eiweißmenge ausbezahlt (Tab. 3.4). 
Tab. 3.4 Milchauszahlungspreis für die Komponenten Grundpreis sowie Fett- und Eiweißpreis in $€$ je Einheit für Fleckvieh, Holstein und Braunvieh im Referenzszenario (OECD/FAO, 2005; Seufferlein, 2005)

\begin{tabular}{lccc}
\hline & Fleckvieh & Holstein & Braunvieh \\
\hline Grundpreis $(€ / \mathrm{kg})$ & 0,00 & 0,02 & 0,00 \\
Fettpreis $(€ / \mathrm{kg})$ & 1,00 & 2,20 & 1,00 \\
Eiweißpreis $(€ / \mathrm{kg})$ & 6,70 & 5,00 & 6,70 \\
\hline
\end{tabular}

Im Fleckvieh- und Braunviehbetrieb wird im Referenzszenario ein ausbezahlter Preis von $0,00 €$ Grundpreis, 1,00€ je kg Fett und 6,70€ je kg Eiweiß unterstellt (Tab. 3.4). Dies spiegelt eine größtmögliche Spreizung zwischen den Merkmalen Fett und Eiweiß wider und beschreibt damit die Entwicklung, die in den Molkereien in den letzten dreißig Jahren stattgefunden hat (Seufferlein, 2005). Der Eiweißpreis hat sich erhöht, der Fettpreis hingegen ist gesunken (Abb. 3.2). Als Richtwert zur Ermittlung der Werte für die Auszahlungspreise gilt der Weltmarktpreis für Butter bzw. Fett und die in Deutschland erzielbaren Preise für Käse (OECD/FAO, 2005). 


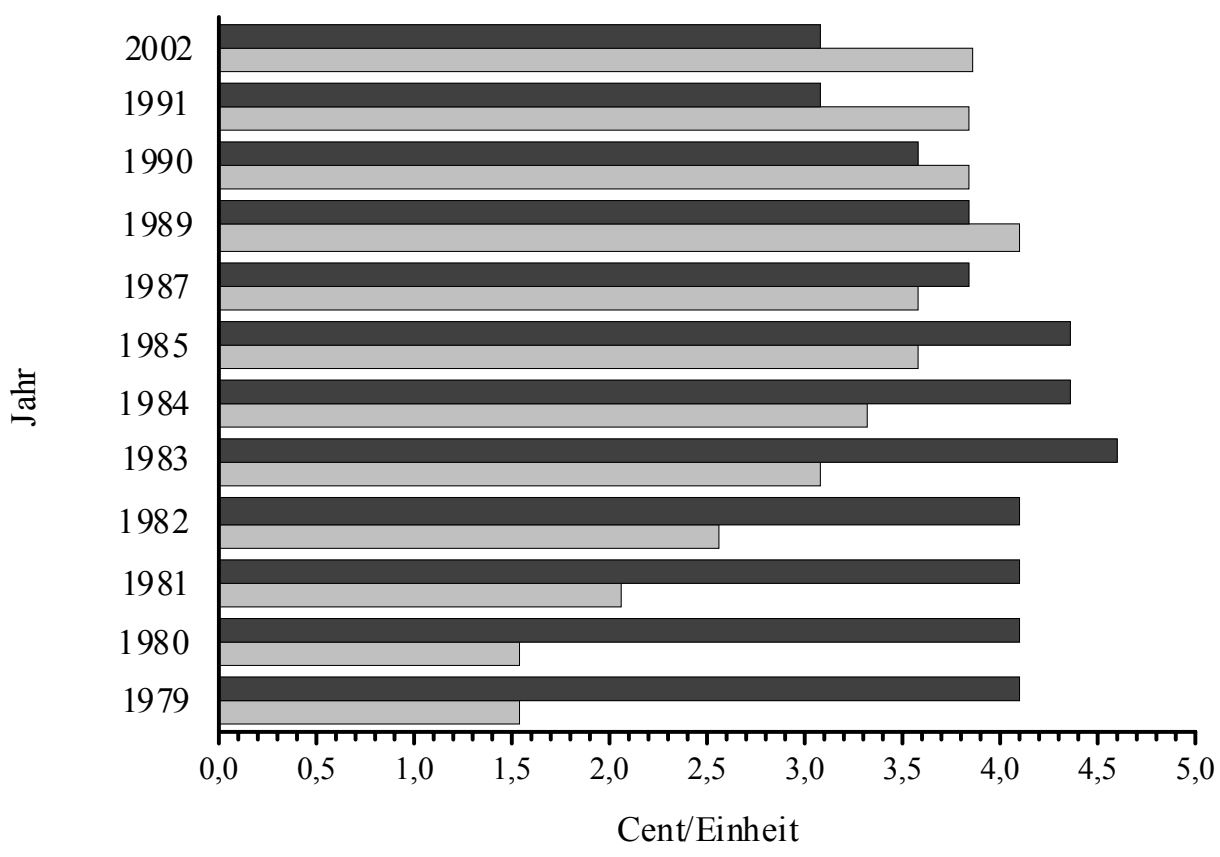

Fetteinheit

Eiweißeinheit

Abb. 3.2 Veränderung der Bezahlung der Fett- und Eiweißeinheiten in Deutschland von 1979 bis 2002 in Cent je Einheit (Seufferlein, 2005)

Für das Vergleichsszenario bei Fleckvieh und Braunvieh werden die gleichen Erlösund Kostenstrukturen unterstellt wie im Referenzszenario. Allerdings wird ein Milchpreis angenommen, der sich aus 0,02€ Grundpreis, 2,70€ je kg Fettmenge und $4,10 €$ je kg Eiweißmenge zusammensetzt. Dies entspricht dem Erzeugerorientierungspreis in Bayern aus dem Jahr 2006. Die Ableitung der wirtschaftlichen Gewichte erfolgt bei Fleckvieh in diesem Szenario mit und ohne Milchquotenbeschränkungen. Bei Braunvieh wird nur die Variante ohne Milchquotenbeschränkung berücksichtigt.

\subsubsection{Futterkosten}

Die Berechnung der Fütterung im Betrieb erfolgt anhand einer Linearen Programmierung. Damit ist gewährleistet, dass immer die günstigste Fütterungsvariante ausgewählt wird. Restriktionen gibt es hinsichtlich der Silage- und Kraftfutteraufnahme. Die im Modell verwendeten Futtermittel sowie deren Inhaltsstoffe und Kosten sind in Tab. 3.5 aufgeführt. 
Tab. 3.5 Angaben zu den unterstellten Futtermitteln je kg Trockensubstanz (Jeroch et al., 1999) und deren aktuelle Preise (Landwirtschaftskammer, 2004)

\begin{tabular}{|c|c|c|c|c|c|}
\hline & \multirow[t]{2}{*}{ Preis in $€$} & \multirow{2}{*}{$\begin{array}{c}\text { Eiweißgehalt in } g \\
\text { Rohprotein }\end{array}$} & \multicolumn{2}{|c|}{ Eiweißgehalt in } & \multirow{2}{*}{$\begin{array}{c}\text { Rohfase } \\
\text { in } \mathrm{g}\end{array}$} \\
\hline & & & MJ NEL & MJ ME & \\
\hline $\mathrm{Heu}$ & 0,10 & 133 & 5,28 & 9,05 & 284 \\
\hline Grassilage & 0,18 & 136 & 6,10 & 10,20 & 213 \\
\hline Maissilage & 0,13 & 131 & 6,48 & 10,80 & 210 \\
\hline Gerstenschrot & 0,14 & 124 & 8,28 & 12,84 & 57 \\
\hline Sojaextraktionsschrot & 0,25 & 398 & 9,90 & 15,88 & 62 \\
\hline
\end{tabular}

Die Preise sind den Berechnungen der Landwirtschaftskammer Schleswig-Holstein entnommen (Landwirtschaftskammer, 2004).

\subsubsection{Kosten der Geburt}

Um bei der Geburt Hinweise auf das Kalbeverhalten zu erlangen, wird der Kalbeverlauf in den Betrieben von den Landwirten festgestellt und der Zuchtwertschätzung zur Verfügung gestellt (Tab. 3.6).

Tab. 3.6 Klassifizierung des Kalbeverlaufs (nach VIT, 2006)

\begin{tabular}{lcr}
\hline Klasse & Bewertung & Interpretation \\
\cline { 2 - 3 } 0 & keine Angabe & nicht beobachtet bzw. keine Angaben verfügbar \\
1 & leicht & ohne Hilfe oder Hilfe nicht nötig, Nachtabkalbung \\
2 & mittel & ein Helfer oder leichter Einsatz mechanischer Zughilfe \\
3 & schwer & mehrere Helfer, mechanische Zughilfe und/oder Tierarzt \\
4 & Operation & Kaiserschnitt, Fetotomie \\
\hline
\end{tabular}

Sowohl bei der Rasse Holstein als auch der Rasse Fleckvieh werden die Geburten nach gleichen Kriterien eingeteilt. 
In Tab. 3.7 sind die einzelnen Kalbeverlaufsklassen für die drei Rassen dargestellt. Holstein hat die meisten Geburten ohne Hilfestellung und die wenigsten Schwergeburten.

Tab. 3.7 Verteilung des Kalbeverlaufs auf die einzelnen Klassen in Prozent und ergänzende Angaben zur Berechnung der durchschnittlichen Kosten je Geburt bei Fleckvieh und Braunvieh (ZuchtData, 2005a) sowie Holstein (Wünsch, 1999)

\begin{tabular}{lccccc}
\hline & \multicolumn{4}{c}{ Kalbeverlaufsklasse (Anteil in \%) } & Kosten je Geburt $(€)$ \\
\cline { 2 - 6 } & 1 & 2 & 3 & 4 & \\
\hline Fleckvieh & 38,50 & 57,00 & 4,30 & 0,20 & 6,92 \\
Holstein & 81,07 & 16,35 & 2,47 & 0,11 & 4,10 \\
Braunvieh & 51,2 & 45,7 & 2,9 & 0,2 & 5,99 \\
AKh & 0,25 & 0,50 & 1,50 & 1,50 & \\
Tierarztkosten $(€)$ & & & 50 & 120 & \\
\hline
\end{tabular}

Es wird unterstellt, dass in den Betrieben der Geburtsbeobachtung ein gewisses Maß an Aufmerksamkeit geschenkt sowie Vor- und Nachsorge betrieben wird, so dass auch eine Kuh mit Leichtgeburt entsprechend betreut wird.

\subsubsection{Fruchtbarkeitskennzahlen}

Im Modell werden die Merkmale Rastzeit, Konzeptionsrate, Verzögerungszeit und die Trächtigkeitsdauer berücksichtigt. Zusätzlich werden die Parameter Lebendgewicht der Kuh und Melkbarkeit im Modell vorgegeben (Tab. 3.8). 
Tab. 3.8 Biologische Parameter im Betrieb bei den Rassen Fleckvieh und Braunvieh (LfL, 2004a) sowie Holstein (Landwirtschaftskammer, 2004)

\begin{tabular}{lcccc}
\hline & Einheit & Fleckvieh & Holstein & Braunvieh \\
\hline Ø Lebendgewicht Kuh & $\mathrm{kg}$ & 750 & 650 & 700 \\
Melkbarkeit & Minuten/100 kg & 4,67 & 3,74 & 4,95 \\
Rastzeit & Tage & 75 & 88 & 80 \\
Trächtigkeitsdauer & Tage & 287 & 282 & 280 \\
\hline
\end{tabular}

In Tab. 3.8 ist die Melkbarkeit in Minuten je $100 \mathrm{~kg}$ Milch angegeben. Dies kommt dadurch zustande, dass neben der eigentlichen Melkbarkeit (Minuten je kg Milch) die Melkstandgröße mit berücksichtigt wird. Dementsprechend wird aufgezeigt, mit welcher Anzahl Melkplätze und welcher Melkbarkeit je Kuh 100 kg Milch ermolken werden können. Dies hat einen Einfluss auf den Betriebsgewinn, da bei entsprechender Melkstandgröße und Melkgeschwindigkeit Arbeitszeit eingespart werden kann.

Die Konzeptionsraten sind an die Daten aus der Zuchtwertschätzung nach Fürst (2006) und Reinhardt (2006) angelehnt (Tab. 3.9, Tab. 3.10, Tab. 3.11).

Tab. 3.9 Konzeptionsraten für Fleckvieh (Fürst, 2006)

\begin{tabular}{lcccccccccc}
\hline \multirow{2}{*}{ Besamungsversuch } & & \multicolumn{8}{c}{ Laktation } \\
\cline { 3 - 10 } & Färsen & 1 & 2 & 3 & 4 & 5 & 6 & 7 & 8 \\
\hline 1 & 0,769 & 0,681 & 0,680 & 0,670 & 0,658 & 0,650 & 0,638 & 0,628 & 0,618 \\
$\geq 2$ & 0,746 & 0,686 & 0,692 & 0,680 & 0,676 & 0,671 & 0,660 & 0,645 & 0,646 \\
\hline
\end{tabular}


Tab. 3.10 Konzeptionsraten für Holstein (Reinhardt, 2006)

\begin{tabular}{lccccccccc}
\hline \multirow{2}{*}{ Besamungsversuch } & & \multicolumn{8}{c}{ Laktation } \\
\cline { 3 - 10 } & Färsen & 1 & 2 & 3 & 4 & 5 & 6 & 7 & 8 \\
\hline 1 & 0,675 & 0,524 & 0,520 & 0,516 & 0,513 & 0,511 & 0,507 & 0,503 & 0,495 \\
$\geq 2$ & 0,655 & 0,524 & 0,520 & 0,510 & 0,513 & 0,511 & 0,510 & 0,500 & 0,495 \\
\hline
\end{tabular}

Tab. 3.11 Konzeptionsrate für Braunvieh (Fürst, 2006)

\begin{tabular}{lccccccccc}
\hline \multirow{2}{*}{ Besamungsversuch } & & \multicolumn{1}{c}{ Laktation } \\
\cline { 3 - 10 } & Färsen & 1 & 2 & 3 & 4 & 5 & 6 & 7 & 8 \\
\hline 1 & 0,750 & 0,635 & 0,642 & 0,641 & 0,636 & 0,624 & 0,606 & 0,599 & 0,598 \\
$\geq 2$ & 0,716 & 0,648 & 0,655 & 0,652 & 0,637 & 0,649 & 0,629 & 0,625 & 0,620 \\
\hline
\end{tabular}

\subsubsection{Somatic Cell Score}

Der Verlust, der auf einem Betrieb aufgrund von Mastitis auftritt, beträgt nach Krömker (2006) $175 €$ je Kuh und Jahr. Darin enthalten sind die Kosten für Behandlung und aufgewendete Arbeitszeit, Tierarztgebühren und der Verlust an Milch für die gesamte Laktation. Da es bei einer Berücksichtigung der reduzierten Milchmenge im Falle einer Mastitis zu einer Doppelzählung des Merkmals Milchleistung käme (Nielsen, 2004), werden im Modell nur die Kosten für die zusätzliche Arbeit und die tierärztliche Behandlung berücksichtigt. So reduzieren sich die Kosten je Mastitisfall je Kuh und Jahr auf 45,95€ bei Holstein. In Tab. 3.12 ist die Verteilung der Zellzahlklassen im Herdendurchschnitt dargestellt. 
Tab. 3.12 Aufteilung der Rassen Fleckvieh, Holstein und Braunvieh auf die Klassen des Somatic Cell Score (LfL, 2004b; ZuchtData, 2005a)

\begin{tabular}{lcccc}
\hline \multirow{2}{*}{ SCS Klasse } & Zellzahl & \multicolumn{3}{c}{ Verteilung } \\
\cline { 3 - 5 } & & Fleckvieh & Holstein & Braunvieh \\
\hline 1 & 25000 & 2,20 & 37,52 & 12,40 \\
2 & 50000 & 21,20 & 22,52 & 20,30 \\
3 & 100000 & 37,50 & 18,99 & 24,50 \\
4 & 12,85 & 13,08 & 21,40 \\
5 & 200000 & 15,85 & 7,41 & 12,50 \\
6 & 400000 & 5,10 & 3,72 & 5,50 \\
7 & 800000 & 2,30 & 1,74 & 2,20 \\
8 & 1600000 & 2,00 & 0,69 & 0,80 \\
9 & 3200000 & 1,00 & 0,13 & 0,10 \\
\hline
\end{tabular}

Die Zuchtwertschätzung für das Merkmal somatischen Zellgehalt erfolgt seit 1996 mit einem Testtagsmodell (VIT, 2006). Die Zellzahlergebnisse werden logarithmisch transformiert zum Linear Somatic Cell Score (3.6):

$$
S C S=\log _{2}(\text { Zellzahl/100) }+3
$$

Die Berücksichtigung der Zellzahl erfolgt anhand des Somatic Cell Score (SCS). Die Transformation richtet sich nach der in der Zuchtwertschätzung verwendeten Formel (VIT, 2006).

\subsubsection{Kosten und Erlöse beim Milchvieh}

Einer der vielfach diskutierten Bereiche im Milchviehbetrieb ist die Arbeitswirtschaft. Um die aufgebrachte Arbeitszeit auch im Modell entsprechend berücksichtigen zu können, gehen die aufgewendeten Arbeitszeiten in alle Teilbereichen des Betriebes in die Berechnung mit ein. 
Die Arbeitszeit im Betrieb sowie die Arbeitskosten (Tab. 3.13) orientieren sich an den Vorgaben der KTBL (KTBL, 2005) bzw. bei den Arbeitskosten der Rassen Fleckvieh und Braunvieh an der Betriebszweigauswertung Bayern (LfL, 2004a).

Tab. 3.13 Arbeitszeit und Arbeitskosten im Betrieb bei den Rassen Fleckvieh, Holstein und Braunvieh (KTBL, 2005)

\begin{tabular}{lcccc}
\hline & Einheit & Fleckvieh & Holstein & Braunvieh \\
\hline Arbeitskosten & $€ /$ AKh & 12,50 & 10,42 & 12,50 \\
Arbeitszeit Nachzuchtfärse & Minuten/Färse und & 1,0 & 1,0 & 1,0 \\
& Tag & & & \\
Arbeitszeit Melken & Minuten/Kuh und Tag & 2,8 & 2,8 & 2,8 \\
Arbeitszeit Management & Minuten/Kuh und Jahr & 2,8 & 2,8 & 2,8 \\
Arbeitszeit Trockenperiode & Minuten/Kuh und Tag & 2,8 & 2,8 & 2,8 \\
\hline
\end{tabular}

Ebenso zu berücksichtigen sind die Tierarztkosten sowie die Stallplatzkosten im Betrieb (Tab. 3.14). Auch hier dienen die Angaben des KTBL (2005) als Orientierung.

Tab. 3.14 Kosten im Betrieb bei den Rassen Fleckvieh, Holstein und Braunvieh (KTBL, 2005)

\begin{tabular}{lcccc}
\hline & Einheit & Fleckvieh & Holstein & Braunvieh \\
\hline Tierarztkosten & $€ /$ Kalbezyklus & 10 & 8 & 10 \\
$\begin{array}{l}\text { Tierarztkosten Nachzucht- } \\
\text { färse }\end{array}$ & $€ /$ Färse & 33 & 33 & 33 \\
$\begin{array}{l}\text { Stallplatzkosten } \\
\begin{array}{l}\text { Stallplatzkosten Nachzucht- } \\
\text { färse }\end{array}\end{array}$ & $€ /$ Färse & 200 & 200 & 200 \\
\hline
\end{tabular}

Maßgeblich zu den Einnahmen im Betrieb zählen die Erlöse von verkauften Tieren zur Zucht bzw. zur Schlachtung. Gerade bei der Rasse Fleckvieh tragen die Schlachterlöse in hohem Maße zum Betriebserfolg bei. 
In Tab. 3.15 sind die Verkaufs- und Schachtdaten für Färsen und Kühe dargestellt.

Tab. 3.15 Verkaufs- und Schlachtdaten der Färsen und Kühe bei den Rassen Fleckvieh und Braunvieh (LfL, 2005) sowie Holstein (Weiss, 2005)

\begin{tabular}{lcccc}
\hline & Einheit & Fleckvieh & Holstein & Braunvieh \\
\hline Ø Verkaufsalter Färse & Tage & 850 & 732 & 859 \\
$\varnothing$ Auktionspreis Färse & $€ /$ Färse & 1393 & 1196 & 1070 \\
$\varnothing$ Auktionspreis Auktionskuh & $€ /$ Kuh & 1245 & 1123 & 1171 \\
$\varnothing$ Schlachtpreis Färse & $€ /$ kg LG & 2,53 & 1,81 & 2,17 \\
Ø Schlachtpreis Kuh & $€ / k g ~ L G$ & 2,18 & 1,67 & 1,74 \\
Ausschlachtung Kuh & $\%$ & 44 & 44 & 44 \\
\hline
\end{tabular}

Für Kühe, die wegen freiwilliger Merzung abgehen, wird angenommen, dass diese Kühe zur weiteren Milchproduktion verkauft werden und dementsprechend einen besseren Preis erzielen können.

\subsubsection{Kosten und Erlöse in der Bullenmast}

Da die Rasse Fleckvieh nach wie vor eine Zweinutzungsrasse ist, werden in der Zuchtwertschätzung die Fleischleistungsmerkmale berücksichtigt. Daher werden wirtschaftliche Gewichte für Fleischleistungsmerkmale Nettozunahme und Handelsklasse abgeleitet. Auch bei Braunvieh erfolgt eine Ableitung der wirtschaftlichen Gewichte für die Merkmale Nettozunahme, Handelsklasse und Ausschlachtung. 
Tab. 3.16 Parameter der Bullenmast bei den Rassen Fleckvieh und Braunvieh (LKV, 2005)

\begin{tabular}{lccc}
\hline & Einheit & Fleckvieh & Braunvieh \\
\hline Start der Mastperiode & Tage & 120 & 90 \\
Dauer der Mastperiode & Tage & 472 & 520 \\
Anfangsgewicht & kg LG & 150 & 82 \\
Endgewicht & kg LG & 670 & 612 \\
Fixkosten für den Maststall & $€ /$ Tier und Tag & 0,38 & 0,95 \\
Tierarztkosten & $€ /$ Tier und Mastperiode & 15 & 50 \\
Sonstige Kosten & $€ /$ Tier und Mastperiode & 18 & 18 \\
Gesamtarbeitszeit Mast & Minuten/Tier und Tag & 1 & 1 \\
\hline
\end{tabular}

Eine täglich Ermittlung der Kosten über die gesamte Mastperiode wird nach Miesenberger (1997) durchgeführt. Anhand des fix vorgegebenen Mastendgewichtes von $689 \mathrm{~kg}$ bzw. $612 \mathrm{~kg}$ und der täglichen Zunahme wird die Länge der Mastperiode ermittelt. In die Berechnung der Bullenmast fließen die Kosten der Kälberfütterungsphase, Stallplatzkosten, Fütterungskosten, Tierarztkosten, Arbeitskosten und sonstige Kosten ein. Die Fütterung erfolgt als Intensivmast mit Maissilage.

Um den durchschnittlichen Preis je kg Schlachtgewicht zu berechnen, werden die Preise je Handelsklasse (EUROP) mit den Anteilen der in die Klassen eingestuften Tiere gewichtet (Miesenberger, 1997). Die in der Ausgangssituation unterstellten Preise und Klassenverteilungen (Tab. 3.17) entsprechen den Auswertungen der BAFF (2006). Es wird eine durchschnittliche Ausschlachtung von 55\% unterstellt. 
Tab. 3.17 Jungbullenpreise und Anteile in den einzelnen Handelsklassen (EUROP) bei Fleckvieh und Braunvieh in Bayern und Baden-Württemberg (BAFF, 2006)

\begin{tabular}{llcccccc}
\hline & & \multicolumn{7}{c}{ Handelsklasse } \\
\cline { 3 - 8 } & & $\mathrm{E}$ & $\mathrm{U}$ & $\mathrm{R}$ & $\mathrm{O}$ & $\mathrm{P}$ & $\varnothing$ Preis \\
& & & & & & & E-P \\
\hline Fleckvieh & $€ / \mathrm{kg}$ & 3,13 & 3,07 & 3,01 & 2,69 & 2,35 & 3,02 \\
& Anteil (\%) & 7,26 & 55,94 & 29,46 & 5,96 & 1,38 & \\
Braunvieh & $€ / \mathrm{kg}$ & 3,19 & 3,13 & 3,08 & 2,74 & 2,44 & 3,07 \\
& Anteil (\%) & 28,72 & 40,41 & 14,18 & 14,59 & 2,10 & \\
\hline
\end{tabular}

\subsubsection{Kosten und Erlöse der Kälberaufzucht}

Es erfolgt zunächst eine intensive Kälberaufzucht, bevor die weiblichen Kälber in den Jungviehbereich wechseln. Bei den Rassen Fleckvieh und Braunvieh verbleiben die männlichen Kälber zur weiteren Bullenmast im Betrieb. Bei der Rasse Holstein werden die männlichen Kälber am Ende der Aufzuchtphase verkauft. In Tab. 3.18 sind die Kosten und Erlöse sowie Parameter der Kälberaufzucht aufgeführt. 
Tab. 3.18 Parameter der Kälberaufzucht bei den Rassen Fleckvieh, Holstein und Braunvieh (KTBL, 2005; Weiss, 2005)

\begin{tabular}{lcccc}
\hline & Einheit & Fleckvieh & Holstein & Braunvieh \\
\hline $\begin{array}{l}\text { Tränkemenge (2. bis 4. Le- } \\
\text { benswoche) }\end{array}$ & Liter & 8 & 6 & 8 \\
Tränkemenge (ab 7. Woche) & Liter & 9 & 7 & 9 \\
Milchaustauscher je Tränke & $\mathrm{kg} / \mathrm{l}$ & 0,1 & 0,1 & 0,1 \\
Preis Milchaustauscher & $€ / \mathrm{kg}$ & 1,15 & 1,15 & 1,15 \\
Preis Kälberstarter & $€ / \mathrm{kg}$ & 0,27 & 0,27 & 0,27 \\
Fixkosten Kälberstall & $€ /$ Tag und Kälberbox & 0,49 & 0,52 & 0,49 \\
Tierarztkosten & $€ /$ Kalb & 22 & 25 & 22 \\
Gesamtarbeitszeit je Kalb & Minuten/Kalb und Tag & 2,0 & 1,7 & 2,0 \\
Totgeburtenrate & $\%$ & 3,9 & 7,9 & 7,2 \\
$\begin{array}{l}\text { Preis je männliches Ver- } \\
\text { kaufskalb }\end{array}$ & $€ / \mathrm{kg} \mathrm{LG}$ & 4,95 & 2,02 & 2,53 \\
$\begin{array}{l}\text { Preis je weibliches Ver- } \\
\text { kaufskalb }\end{array}$ & $€ / \mathrm{kg} \mathrm{LG}$ & 3,05 & 1,10 & 1,73 \\
$\begin{array}{l}\text { Verkaufsgewicht männliches } \\
\text { Kalb }\end{array}$ & $\mathrm{kg} \mathrm{LG}$ & 90 & 60 & 62 \\
$\begin{array}{l}\text { Verkaufsgewicht weibliches } \\
\text { Kalb }\end{array}$ & $\mathrm{kg} \mathrm{LG}$ & 85 & 55 & 60 \\
\hline
\end{tabular}

In der Jungviehaufzucht werden die Stallplatz-, Fütterungs- und Arbeitskosten täglich ermittelt, von der Geburt des Kalbes bis zum Verkauf oder zur Abkalbung der Färse. Die Färsen, die zur Zucht verkauft werden, verlassen den Betrieb 60 Tage vor dem errechneten Abkalbetermin. 


\subsection{Ableitung der wirtschaftlichen Gewichte}

Der Grenznutzen eines Merkmals beschreibt die Änderung des Gewinns eines Unternehmers, welches sich aus der genetisch bedingten Produktionsveränderung des Merkmals um eine Einheit, bei Konstanthaltung aller übrigen Merkmale des Gesamtzuchtwertes, ergibt. Um eine Vergleichbarkeit über alle Merkmale zu erhalten, wird der Grenznutzen mit der genetischen Standardabweichung des jeweiligen Merkmals zum wirtschaftlichen Gewicht zusammengefasst (Miesenberger, 1997).

Im Folgenden werden die Merkmale beschrieben, für die wirtschaftliche Gewichte abgeleitet worden sind. Die Ableitung erfolgt in Anlehnung an Miesenberger (1997).

\subsubsection{Milchleistungsmerkmale}

Die Milchleistungsmerkmale teilen sich in die Merkmale Milchträger, Fettmenge und Eiweißmenge auf.

\section{Milchträger (fett- und eiweißfreie Milch)}

Die Berechnung des wirtschaftlichen Gewichtes für das Merkmal Milchträger erfolgt über die Erhöhung der täglichen Milchmenge. Dabei muss die Steigerung um einen täglich gleichen Betrag über die gesamte Laktation generiert werden. Dadurch wird gewährleistet, dass die Laktationskurve über die gesamte Länge der Laktation konstant bleibt. Werden bei der Ableitung der Gewichte die Merkmale Fett- und Eiweißmenge ebenfalls berücksichtigt, müssen die Inhaltsstoffe in der Milch entsprechend reduziert werden, um eine Doppelberücksichtigung der beiden Merkmale, zum einen über die Milch, zum anderen über die Inhaltsstoffe, zu vermeiden. Als Leistungsunterschied zwischen den beiden Leistungsstufen wird eine Milchleistungssteigerung von $0,01 \mathrm{~kg}$ pro Herdendurchschnittskuh und Tag angenommen.

\section{Fettmenge}

Die Veränderung im Merkmal Fettmenge erfolgt über eine Veränderung der Parameter in der WOOD-Gleichung, die den Laktationskurvenverlauf in den Merkmalen Milch, Fett und Eiweiß nach Wood (1967) beschreibt. Die Erhöhung beträgt 0,1 Prozentpunkte je Herdendurchschnittskuh und Jahr. Es ist möglich, die wirtschaftlichen Gewichte für die Fett- wie auch die Eiweißmenge über zwei unterschiedliche Varianten abzuleiten. 
In der ersten Variante werden die Merkmale über die Inhaltsstoffe verändert. Dies ist notwendig, wenn auch die Milchleistung bzw. der Milchträger als Merkmal in den Gesamtzuchtwert einfließt. Die Inhaltsstoffe werden verändert, während die Milchmenge konstant gehalten wird. In der zweiten Variante können die Fett- und Eiweißmenge über die Milchmenge bei Konstanthaltung der Inhaltsstoffe erhöht werden. Die Inhaltsstoffe bleiben in diesem Fall unverändert, aber die Fett- bzw. Eiweißmenge variiert. Diese Art der Ableitung ist nur zulässig, wenn das Merkmal Milchmenge bzw. Milchträger nicht im Gesamtzuchtwert berücksichtigt wird.

\section{Eiweißmenge}

Die Ableitung der wirtschaftlichen Gewichte für das Merkmal Eiweißmenge erfolgt in der gleichen Weise wie für das Merkmal Fettmenge. Auch hier können beide Varianten der Veränderung des Merkmals angewendet werden.

\subsubsection{Fleischleistungsmerkmale}

Wirtschaftliche Gewichte für die Fleischleistungsmerkmale werden bei Fleckvieh und Braunvieh abgeleitet. Da Fleckvieh nach wie vor als Zweinutzungsrasse gilt, gehen die Fleischleistungsmerkmale in die Zuchtwertschätzung mit ein. Ziel ist es, die wirtschaftliche Bedeutung der Fleischleistung entsprechend bei der Selektion und Zuchtverwendung zu berücksichtigen (Blaas und Sölkner, 1996).

Blaas und Sölkner (1996) weisen darauf hin, dass nicht das Verhältnis der Preise von Milch zu Fleisch das entscheidende Kriterium für die Gewichtung von Milch- zu Fleischleistung darstellt, sondern die Höhe und Zusammensatzung der Kosten für die Milch- und Rindfleischproduktion die entscheidenden Faktoren sind. Die Merkmale der Fleischleistung werden sowohl im Feld als auch auf Prüfstationen, als Eigenleistungs- und Nachkommenprüfung, aufgenommen (ZuchtData, 2005b). Die für die einzelnen Merkmale berechneten Grenznutzen wurden auf eine Herdendurchschnittskuh und Jahr bezogen und aufgrund der Vergleichbarkeit mit ihrer genetischen Standardabweichung multipliziert. Es werden bei Fleckvieh Zuchtwerte für die Merkmale Nettozunahme und Schlachtkörperkonformation berechnet (Schild und Niebel, 1998). In Deutschland wurden zusätzlich Zuchtwerte für das Merkmal Fleischanteil, in Österreich für das Merkmal Ausschlachtung berechnet. Seit November 2006 werden in der Zuchtwertschätzung die Merkmale Nettozunahme, Handelsklasse und Ausschlachtung in der gemeinsamen Zuchtwertschätzung von Deutschland und Österreich beim Fleckvieh verwendet. Auf das Merkmal Fleischanteil wird aufgrund der fehlenden Datengrundlage komplett verzichtet. 


\section{Nettozunahme}

Im Gegensatz zu Miesenberger (1997) wird anstelle des Merkmals tägliche Zunahme ein wirtschaftliches Gewicht für das Merkmal Nettozunahme abgeleitet. Die Ableitung des wirtschaftlichen Gewichtes erfolgt über eine Veränderung der Parameter in der Gompertz-Funktion (Fitzhugh, 1976).

\section{Ausschlachtungsprozente}

Die Ausschlachtungsprozente werden im Gesamtzuchtwert der Rasse Braunvieh berücksichtigt. Im Rahmen dieser Arbeit wird der wirtschaftliche Wert dieses Merkmals berechnet. Die Leistungssteigerung wird über eine Veränderung der Ausschlachtungsprozente um einen Prozentpunkt realisiert.

\section{EUROP-Handelsklasse}

Das Merkmal EUROP-Handelsklasse ist ein kategorisches Merkmal, das die Ausformung des Schlachtkörpers beschreibt. Um ein wirtschaftliches Gewicht für das Merkmale Handelsklasse abzuleiten, werden die Anteile der Schlachtbullen in den einzelnen EUROP-Klassen nach Miesenberger (1997) variiert.

\subsubsection{Funktionale Merkmale}

Die wirtschaftlichen Gewichte für die funktionalen Merkmale werden ebenfalls je Herdendurchschnittskuh und Jahr berechnet. Sie basieren auf Merkmalsveränderungen in allen Laktationen (Miesenberger, 1997).

\section{Nutzungsdauer}

Seit 1996 wird das Merkmal funktionale Nutzungsdauer in der Zuchtwertschätzung berücksichtigt (VIT, 2006). Die Ableitung der wirtschaftlichen Gewichte für Nutzungsdauer erfolgt bei gleichzeitiger Änderung der Wahrscheinlichkeit für unfreiwillige Merzung in allen Laktationen um jeweils einen Prozentpunkt. Dabei bleiben die Wahrscheinlichkeiten, wegen eines anderen Abgangsgrundes aus der Herde auszuscheiden, in der Ausgangssituation konstant. Durch die veränderten Abgangsraten kommt es zu einer Verschiebung in den einzelnen Herdenklassen und damit zu einer Veränderung in der Länge der Nutzungsdauer. 


\section{Fruchtbarkeit}

Trotz niedriger Heritabilitäten der Fruchtbarkeitsmerkmale ist eine züchterische Verbesserung der Fruchtbarkeit als sinnvoll zu erachten, da diese durch eine Senkung der Kosten in Milchviehbetrieben erheblich zum wirtschaftlichen Erfolg der Rinderzucht beiträgt (Fürst und Gredler, 2006). Die Berechnung der wirtschaftlichen Gewichte erfolgt für das Merkmal Fruchtbarkeit über eine Veränderung der Konzeptionsrate um einen Prozentpunkt. Alle übrigen Annahmen bleiben in der Ausgangssituation konstant.

\section{Kalbeverlauf}

Das Merkmal Kalbeverlauf wird in der Zuchtwertschätzung über die Beschreibung in den einzelnen Kalbeklassen mit einbezogen (VIT, 2006). Die Berechnung der wirtschaftlichen Gewichte erfolgt analog zu denen im Merkmal Handelsklasse über eine Veränderung in der Klassenverteilung unter Annahme einer standardisierten Normalverteilung (Miesenberger, 1997).

\section{Totgeburtenrate}

Die Totgeburtenrate wird in der Zuchtwertschätzung als binäres Merkmal behandelt (VIT, 2006). Eine Abkalbung wird als Totgeburt eingeordnet, wenn das Kalb tot geboren wird oder innerhalb von 48 Stunden verendet. Es wird angenommen, dass die Ausprägung des Merkmals Totgeburtenrate in allen Laktationsklassen identisch ist, obwohl dies in der Realität nicht zutrifft. Die Ableitung der wirtschaftlichen Gewichte erfolgt über eine Veränderung der Totgeburtenrate um einen Prozentpunkt in allen Laktationen (Miesenberger, 1997).

\section{Persistenz}

Das Merkmal Persistenz ist von unterschiedlichen Definitionen geprägt. Im Allgemeinen wird darunter die Fähigkeit einer Kuh verstanden, ihre Milchleistung über eine Laktation hinweg zu halten (Swalve und Gengler, 1999). Die Persistenz hat einen gewissen Einfluss auf die Kosten der Fütterung. Dekkers et al. (1998) weisen darauf hin, dass das wirtschaftliche Gewicht der Persistenz unabhängig von der Laktationsleistung betrachtet werden muss, solang die Milchleistung im Gesamtzuchtwert berücksichtigt ist. 
Im Programm wird das Merkmal entsprechend der Definition von Sölkner und Fuchs (1987) eingebunden (Miesenberger, 1997). Beim Vergleich von verschiedenen Maßen für den Verlauf der Laktationskurve stellt sich heraus, dass es günstig ist, mit den Standardabweichungen in den Einzelkontrollen zu arbeiten, um das wirtschaftliche Gewicht für dieses Merkmal in angemessener Weise ableiten zu können. Dabei wird, wie bei Miesenberger (1997), der Laktationskurvenverlauf in allen Laktationen durch die Variation der Wood-Parameter bei konstantem Milchleistungspotential einer Standardlaktation verändert. In der ersten, zweiten und dritten Laktation wird die durchschnittliche Standardabweichung der Einzelkontrollen jeweils um eine genetische Standardabweichung erniedrigt, was in einer besseren Persistenz resultiert. Die dafür verwendeten Wood-Parameter wurden iterativ ermittelt.

\section{Somatic Cell Score (SCS)}

Das Merkmal Somatic Cell Score (SCS) ist ebenfalls ein kategorisches Merkmal. Die Einteilung des SCS erfolgt in Klassen wie unter 3.2.6 beschrieben. Die Ableitung der wirtschaftlichen Gewichte wird über eine Verschiebung der Anteile um eine Standardabweichung in den einzelnen Klassen realisiert, wodurch eine Verbesserung des durchschnittlichen SCS erzielt wird.

\section{Melkbarkeit}

Die Ableitung der wirtschaftlichen Gewichte für das Merkmal Melkbarkeit erfolgt über die Veränderung des durchschnittlichen Minutengemelks je Herdendurchschnittskuh und Jahr.

\subsubsection{Problematik Exterieur}

Die in der Zuchtwertschätzung verwendeten Daten bei der Rasse Holstein basieren auf der linearen Beschreibung und Bewertung von Kühen in der ersten Laktation (VIT, 2006). Die Zuchtwertschätzung erfolgt über Einzelzuchtwerte für die Linearmerkmale, die wiederum zu Indices für die Merkmalskomplexe Milchtyp, Körper, Fundament und Euter berechnet werden. Diese werden schließlich im Relativzuchtwert Euter (RZE) miteinander kombiniert. Bei Fleckvieh werden die linearen Merkmale zu den Merkmalsbereichen Rahmen, Bemuskelung, Form und Euter zusammengefasst (Fürst und Krogmeier, 2004). Hier erfolgt ebenfalls eine Beschreibung bzw. Bewertung der Einzelmerkmale von zufällig ausgewählten Töchtern von Bullen aus dem Prüfeinsatz. Seit 2002 wird in der gemeinsamen Zuchtwertschätzung von Deutschland und Österreich das Exterieur als Hilfsmerkmal für die Nutzungsdauer 
verwendet. Die Zuchtwertschätzung für die Merkmale des Exterieurs wird mit den neusten Methoden durchgeführt und liefert daher wichtige Ergebnisse für Selektionsund Anpaarungsentscheidungen (Fürst und Krogmeier, 2004).

Die Ableitung eines wirtschaftlichen Gewichtes für das Merkmal Exterieur wurde bisher nicht vorgenommen (Sölkner et al., 1999). Seit den neunziger Jahren wird eine Zuchtwertschätzung durchgeführt, jedoch fehlen bisher Ansätze, um den Einfluss des Exterieurs auf die Wirtschaftlichkeit im Betrieb angemessen zu beurteilen. Aufgrund des hohen Anteils des Merkmals Exterieur im Gesamtzuchtwert in der internationalen Zuchtwertschätzung (Fürst, 1999) werden Möglichkeiten gesucht, um Grenznutzen bzw. wirtschaftliche Gewichte für dieses Merkmal abzuleiten (Fürst-Waltl et al., 2004). Bei der Rasse Fleckvieh wird das Exterieur bisher nicht im Gesamtzuchtwert berücksichtigt. Bei der Rasse Holstein wird das relative wirtschaftliche Gewicht approximativ festgesetzt (Schierenbeck, 2006). Da ein Merkmal bei der Berechnung wirtschaftlicher Gewichte nicht doppelt berücksichtigt werden darf, ist es schwierig, den Beitrag des Exterieurs zur Wirtschaftlichkeit des Betriebes zu bestimmen. Durch den Beitrag des Exterieurs zur Verbesserung der Nutzungsdauer ist dieses bereits im wirtschaftlichen Gewicht der Nutzungsdauer enthalten und dieser Beitrag darf nicht in ein wirtschaftliches Gewicht für Exterieur mit eingehen. In mehreren Projekten wurde daher in Deutschland und Österreich der Einfluss der Exterieurmerkmale auf den Auktions- bzw. Marktpreis von Färsen untersucht (Fürst-Waltl et al., 2004; Krogmeier et al., 2006; Schierenbeck, 2006), um den Beitrag des Merkmals Exterieur zu bestimmen, der nicht über das wirtschaftliche Gewicht der Nutzungsdauer mit berücksichtigt wird. Fürst-Waltl et al. (2004) stellen einen deutlichen Einfluss des Exterieurs auf die Versteigerungspreise von Färsen auf österreichischen Auktionen fest. Daher leiten sie wirtschaftliche Gewichte für die Exterieurhauptnoten ab. Krogmeier et al. (2006) berechnen Grenzerträge für die Exterieurhauptnoten. Diese stellen den zusätzlichen Ertrag für eine durchschnittliche Färse bei einer Versteigerung dar, wenn sich das Populationsniveau um eine Standardabweichung erhöht. Weiterhin handelt es sich bei den Grenzerträgen nicht um einen klassischen Grenznutzen oder ein wirtschaftliches Gewicht, weil die Realisierungswahrscheinlichkeit nicht auf eine Herdendurchschnittskuh bezogen ist. Es wird vielmehr die Wertschätzung, die ein Käufer den einzelnen Informationen beimisst, ermittelt. Allerdings geben die Autoren auch zu bedenken, dass sich die Gewichtung, die dem Merkmal Exterieur bei Einbeziehung in den Gesamtzuchtwert zukommen müsste, mit ihrer Untersuchung nicht zufriedenstellend ableiten lässt.

Schierenbeck (2006) zeigt in einer Varianzanalyse anhand von Auktions-, Abstammung- und Leistungsdaten aus dem Zuchtgebiet der Nordrind, dass ein signifikanter Einfluss der Färsenleistung und der Exterieurbewertung auf die Auktionspreise be- 
steht. Die Färsenleistung ist der wichtigste Einflussfaktor, gefolgt von der Euter- und Fundamentnote. Anhand der Ergebnisse scheint es durchaus möglich, ein wirtschaftliches Gewicht für das Merkmal Exterieur abzuleiten. Aufgrund dieser Annahmen erfolgt die Ableitung des wirtschaftlichen Gewichts für das Merkmal Exterieur über die Veränderung der Verkaufspreise für Färsen bei den Rassen Holstein und Braunvieh. Es wird davon ausgegangen, dass ein erhöhter Verkaufspreis auf eine verbesserte Gesamtnote im Exterieur zurückzuführen ist. Die Realisierungswahrscheinlichkeit eines Auktionsverkaufs wird über die Realisierungswahrscheinlichkeit im Modell eingebunden.

Weiterhin ermittelt Schierenbeck (2006) in seiner Arbeit Korrelationen zwischen dem Verkaufspreis der Färse und der Exterieurgesamtnote von $r_{g}=0,411$. Alle vier Exterieurkomplexe üben einen signifikanten Einfluss auf den Verkaufspreis aus. Das wichtigste Merkmal bei der Preisfindung bleibt jedoch die Milchleistung der Färse. Je 1 kg Milchleistung je Färse konnte ein Mehrerlös von 20,47€ erzielt werden, je Punkt Euternote ein Mehrerlös von 17,91€ und je Punkt Fundamentnote 11,25€. Fürst-Waltl et al. (2004) berechnen ein wirtschaftliches Gewicht für Exterieur für Fleckvieh von 6 bis $10 €$. Krogmeier et al. (2006) zeigen, dass bei gleichzeitiger Verbesserung aller Exterieurmerkmale um eine genetische Standardabweichung der Erlös beim Fleckvieh um 35,19€ erhöht wird. Allerdings geben sie zu bedenken, dass die Auswertung sich nicht auf eine Herdendurchschnittskuh bezieht und eine entsprechende Realisierungswahrscheinlichkeit noch zu berücksichtigen wäre. Damit beträgt der wirtschaftliche Wert der Exterieurmerkmale ca. 28\% der Tagesmilchmenge ohne Berücksichtigung der Realisierungswahrscheinlichkeit. Es bleibt allerdings zu berücksichtigen, dass nicht jeder Milchviehbetrieb Zuchtvieh vermarktet und dies auch nicht in gleichem Ausmaß geschieht. Daher muss eine bestimmte Realisierungswahrscheinlichkeit für Zuchtviehverkäufe angenommen werden, um den wirtschaftlichen Einfluss des Exterieurs zu quantifizieren (Fürst-Waltl et al., 2004; Krogmeier et al., 2006; Schierenbeck, 2006).

Bei der Ableitung wirtschaftlicher Gewichte für den Gesamtzuchtwert dürfte neben dem Erlös aus Zuchtviehverkäufen nur der wirtschaftliche Nutzen berücksichtigt werden, der über bereits in anderen Teilzuchtwerten einbezogene Effekte hinausgeht (Krogmeier et al., 2006). Der Zusatznutzen ist allerdings schwer zu fassen und in Modellkalkulationen einzubeziehen. Da das Exterieur aber nach wie vor große Bedeutung in der deutschen Holsteinpopulation besitzt und dies in angemessener Weise berücksichtigt werden soll (Swalve und Höver, 2003), stellt sich die Frage, wie mit dem von der direkten ökonomischen Seite geringen wirtschaftlichen Gewichtes des Exterieurs und der geforderten hohen Gewichtung im Gesamtzuchtwert umgegangen werden soll. Fürst-Waltl (2004) schlägt vor, dass Exterieur bei Fleckvieh in einer 
Höhe von 4 bis 7\% im Gesamtzuchtwert einzubeziehen. Anhand der Ergebnisse von Schierenbeck (2006) erfolgt eine Approximation über die ermittelten Mehrerlöse je verbessertem Exterieurpunkt und anhand der daraus berechneten Indexgewichte werden verschiedene Szenarien zur Einbindung des Merkmalskomplexes Exterieur in den Gesamtzuchtwert vorgeschlagen.

\subsection{Ableitung optimaler Indexgewichte (b-Werte)}

Um Indexgewichte berechnen zu können, ist es nötig, die wirtschaftlichen Gewichte der im Gesamtzuchtwert enthaltenen Merkmale zu kennen. Des Weiteren benötigt man Informationen zu der genetischen Korrelationsmatrix ( $\left.\boldsymbol{r}_{g}\right)$ (Tab. 3.19). Die optimalen Indexgewichte für die zuvor abgeleiteten wirtschaftlichen Gewichte werden mit einer Methode von Reinhardt (2006) berechnet.

Tab. 3.19: Genetische Korrelationsmatrix $\left(r_{g}\right)$ für die einzelnen Teilzuchtwerte (VIT, 2006)

\begin{tabular}{lccccc}
\hline & $R Z M$ & $R Z E$ & $R Z S$ & $R Z N$ & $R Z Z$ \\
\hline RZM & 1,00 & 0,15 & $-0,10$ & $-0,10$ & $-0,05$ \\
RZE & 0,15 & 1,00 & 0,10 & 0,20 & 0,00 \\
RZS & $-0,10$ & 0,10 & 1,00 & 0,00 & 0,10 \\
RZN & $-0,10$ & 0,20 & 0,00 & 1,00 & 0,20 \\
RZZ & $-0,05$ & 0,00 & 0,10 & 0,20 & 1,00 \\
\hline
\end{tabular}

Die genetische Korrelation zwischen RZN und RZS ist in der Gewichtung des RZN berücksichtigt und geht deshalb mit einem Wert von Null in die Berechnung ein (Tab. 3.19).

Um Aussagen über die Indexgewichte geben zu können, werden die b-Werte sowohl für die Situation berechnet, in der Sicherheiten vorliegen, die einem Testbullen nach Testeinsatz entsprechen und die Situation, in der Sicherheiten für einen geprüften Vererber in die Berechnung mit eingehen (Tab. 3.20). 
Tab. 3.20 Sicherheiten der Teilzuchtwerte bei einem Testbullen nach Testeinsatz und bei einem geprüften Vererber (Reinhardt, 2006)

\begin{tabular}{lccccc}
\hline & $R Z M$ & $R Z E$ & $R Z S$ & $R Z N$ & $R Z Z$ \\
\hline Testbulle nach Testeinsatz & 0,90 & 0,70 & 0,75 & 0,65 & 0,60 \\
geprüfter Vererber & 0,98 & 0,85 & 0,80 & 0,80 & 0,80 \\
\hline
\end{tabular}




\section{$4 \quad$ Ergebnisse}

In diesem Kapitel werden die Ergebnisse der Modellkalkulation vorgestellt. Die dargestellten Preis-, Kosten- und Managementannahmen werden unverändert übernommen und aus dem Referenzszenario heraus werden die wirtschaftlichen Gewichte für die einzelnen Rassen abgeleitet. Darüber hinaus erfolgt eine Untersuchung unterschiedlicher Einflussfaktoren auf die wirtschaftlichen Gewichte innerhalb der Rassen.

Die Referenzszenarien sind möglichst realitätsnah beschrieben, um ein Abbild eines Durchschnittsbetriebes in Deutschland und Österreich geben zu können.

\subsection{Wirtschaftliche Gewichte}

Es werden sowohl Grenznutzen je Merkmalseinheit als auch wirtschaftliche Gewichte je genetischer Standardabweichung $\left(\sigma_{\mathrm{a}}\right)$ dargestellt. Zum einen ist der Bezug zum Merkmal beim Grenznutzen realer, um jedoch eine Vergleichbarkeit der Ergebnisse zu ermöglichen, werden diese je genetischer Standardabweichung ausgewiesen. Die Ableitung erfolgt für die Rassen Holstein, Fleckvieh und Braunvieh. Als Bezugspunkt bei den einzelnen Rassen dient jeweils das Referenzszenario mit den für das Modell beschriebenen Eingabeparametern.

Bei Holstein erfolgt die Ableitung von Grenznutzen und wirtschaftlichen Gewichten im Referenzszenario mit und ohne Milchquotenbeschränkungen, zusätzlich wird die Frage der Fett- zu Eiweißgehalt-Gewichtung betrachtet und der Einfluss der Gewichtung auf die Zusammensetzung des Gesamtzuchtwertes untersucht. Bei Fleckvieh werden Grenznutzen und wirtschaftlichen Gewichte dargestellt, es erfolgt hier eine Untersuchung verschiedener Quoten- und Milchauszahlungspreisszenarien. Auch hier wird weiterführend der Einfluss verschiedener Herdenparameter auf die wirtschaftlichen Gewichte analysiert. Bei Braunvieh wird lediglich die Ableitung von Grenznutzen und wirtschaftlichen Gewichten, auch unter der Annahme verschiedener Milchpreis- und Quotenszenarien, durchgeführt. 


\subsubsection{Holstein}

In Tab. 4.1 sind die genetischen Standardabweichungen $\left(\sigma_{a}\right)$ der jeweiligen Merkmale bei der Rasse Holstein aufgeführt.

Tab. 4.1 Genetische Standardabweichung der einzelnen Merkmale bei Holstein (Reinhardt, 2006)

\begin{tabular}{lcc}
\hline & Merkmalseinheit & Genetische Standardabweichung \\
\hline Milchträger & $\mathrm{kg}$ & 561 \\
Fettmenge & $\mathrm{kg}$ & 23,1 \\
Eiweißmenge & $\mathrm{kg}$ & 16,7 \\
Nutzungsdauer & Tage & 180 \\
Fruchtbarkeit & $\%$ & 5,0 \\
Kalbeverlauf & Klasse & 1,0 \\
Totgeburtenrate & $\%$ & 5,0 \\
Persistenz & $\sigma$ & 1,0 \\
SCS & Klasse & 0,38 \\
Melkbarkeit & kg/Min & 0,15 \\
Exterieur & Punkte & 2,28 \\
\hline
\end{tabular}

Die genetischen Standardabweichungen geben Auskunft über die Streuung der Merkmale in der Population. Anhand dessen kann die Veränderung der Merkmale besser eingeordnet werden.

In Abb. 4.1 sind die Grenznutzen der Merkmale je Einheit bei der Rasse Holstein dargestellt. Diese zeigen die Veränderungen im Gewinn des Betriebes, wenn die Merkmale durch züchterische Maßnahmen um eine Einheit verbessert werden. Der Grenznutzen bzw. das wirtschaftliche Gewicht der einzelnen Merkmale werden, wie in Kapitel 3.1.1 beschrieben, berechnet. Zusätzlich sind zu den einzelnen Merkmalen die zugehörigen Standardabweichungen dargestellt. 


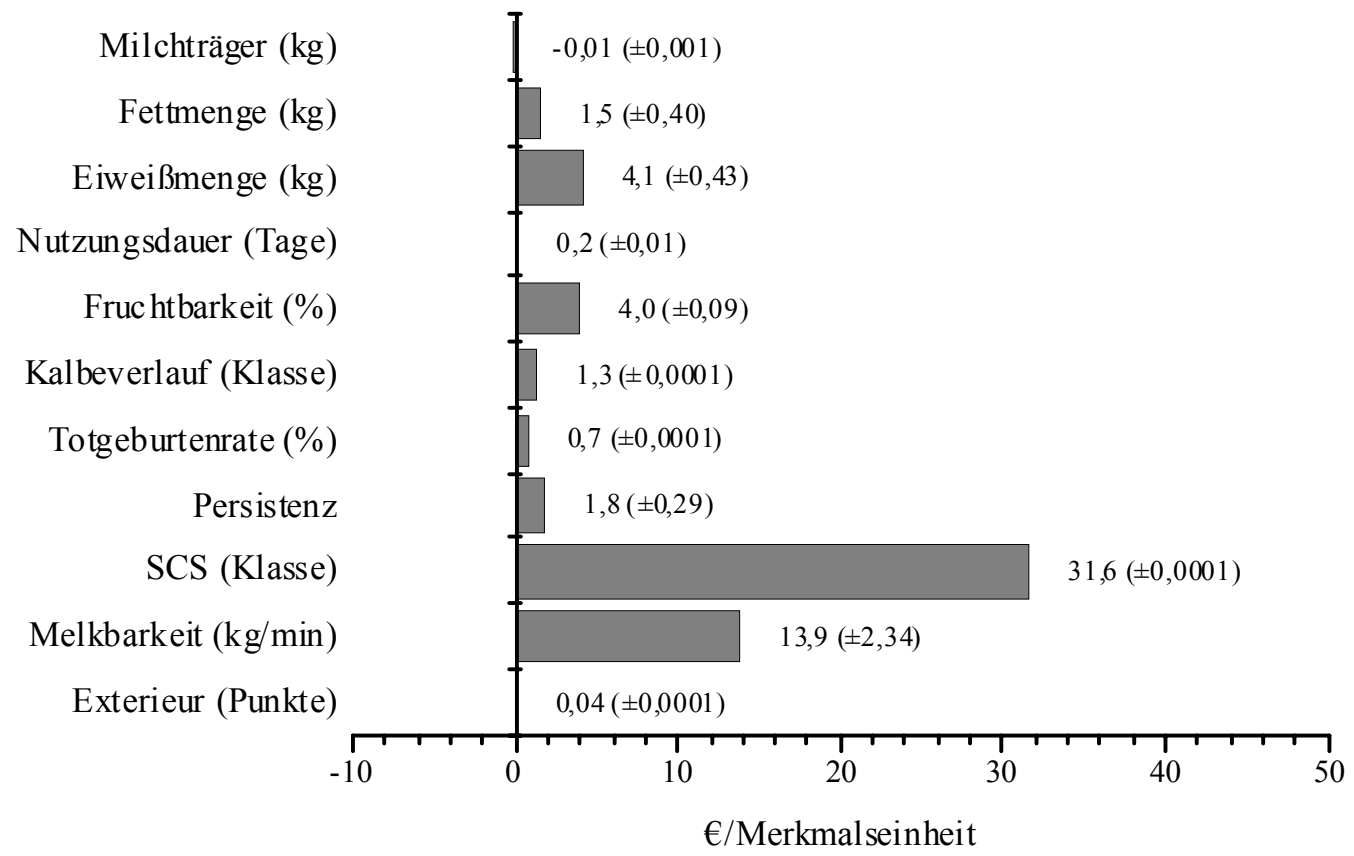

Abb.4.1 Grenznutzen ( \pm Standardabweichung) der einzelnen Merkmale in $€$ je Merkmalseinheit bei Holstein im Referenzszenario ohne Milchquotenbeschränkung

Die Milchleistungsmerkmale Milchträger (fett- und eiweißfreie Fraktion), Fett- und Eiweißmenge haben einen Grenznutzen von $-0,01 €$ je $\mathrm{kg}, 1,6 €$ je $\mathrm{kg}$ und $4,1 €$ je $\mathrm{kg}$. Durch die geringe Bezahlung des Milchträgers im Milchauszahlungspreis kommt es zu einem negativen Grenznutzen in diesem Merkmal. Der Grenznutzen für die Eiweißmenge ist 2,65-mal höher als jener der Fettmenge, bei einem Bezahlungsverhältnis von 1:2,27 im Milchauszahlungspreis. Der Grenznutzen der Nutzungsdauer liegt bei 6,04€ je Monat, d. h. einer Verbesserung des Merkmals um einen Monat würde den Gewinn um etwa $6 €$ je Kuh und Jahr steigern. Die Verbesserung der Fruchtbarkeit würde einen Zusatzgewinn von $4 €$ je \% bewirken, bei den Merkmalen Kalbeverlauf, Totgeburtenrate und Persistenz liegt dies im Bereich zwischen ein bis zwei Euro. Der Grenznutzen der SCS liegt bei $31 €$ je SCS-Klasse und $14 €$ je kg pro Minute für das Merkmal Melkbarkeit. Für das Merkmal Exterieur ist der Grenznutzen mit $0,04 €$ je Exterieurgesamtpunkt niedrig.

Wie in Abb. $4.1 \mathrm{zu}$ sehen ist, sind die Standardabweichungen bei den Milchleistungsmerkmalen und der Melkbarkeit am höchsten. Dieses ist, wie bei den übrigen Rassen auch, dadurch zu erklären, dass der Milchpreis und die Milchleistung diejeni- 
gen Merkmale sind, die zur Berechnung der Mittelwerte der wirtschaftlichen Gewichte variiert werden (vgl. Kapitel 3.1.1). Daher kommt es bei den Grenznutzen dieser Merkmale auch zu den größten Abweichungen.

Ein wichtiges Entscheidungskriterium, gerade vor dem Hintergrund der derzeitigen Milchpreisdiskussion, ist die Gewichtung der Merkmale Fett- und Eiweißmenge zueinander. Die beiden Merkmale stehen in einem Verhältnis von 1:2,65 zueinander bei einer Fett-zu-Eiweiß-Bezahlung von 2,20€ je Fetteinheit zu 5,00€ je Eiweißeinheit (Abb. 4.2).

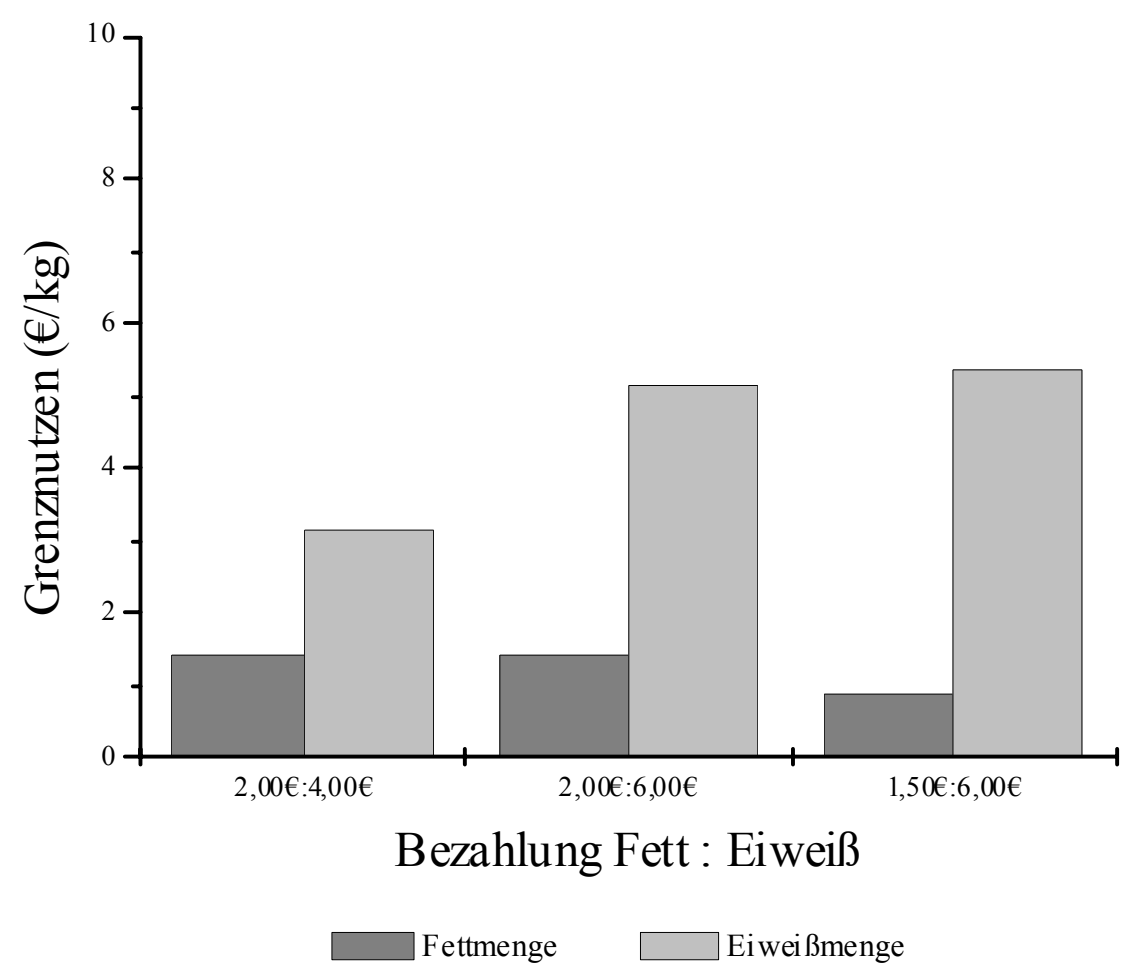

Abb. 4.2 Grenznutzen für die Fett- und Eiweißmenge in Abhängigkeit vom Bezahlungssystem bei der Rasse Holstein (in $€$ je kg)

Wählt man die Bezahlungsverhältnisse von Fettmenge zu Eiweißmenge von 1:2, 1:3, und 1:4 (Abb. 4.2), so erhält man wirtschaftliche Gewichte, die ein Verhältnis von 1:2, 1:4 und 1:6 widerspiegeln. Es wird deutlich, dass sich mit zunehmender Spreizung des Auszahlungspreises für Fett und Eiweiß auch das Verhältnis der Grenznutzen zueinander erhöht. Unter Annahmen eines zukünftigen Milchpreises muss dementsprechend das Verhältnis von Fett- zu Eiweißmenge gewählt werden. 
Um nun die Merkmale nicht nur für sich genommen betrachten zu können, werden diese in $€$ je genetischer Standardabweichung ausgewiesen. Die wirtschaftlichen Gewichte sind in Abb. 4.3 dargestellt.

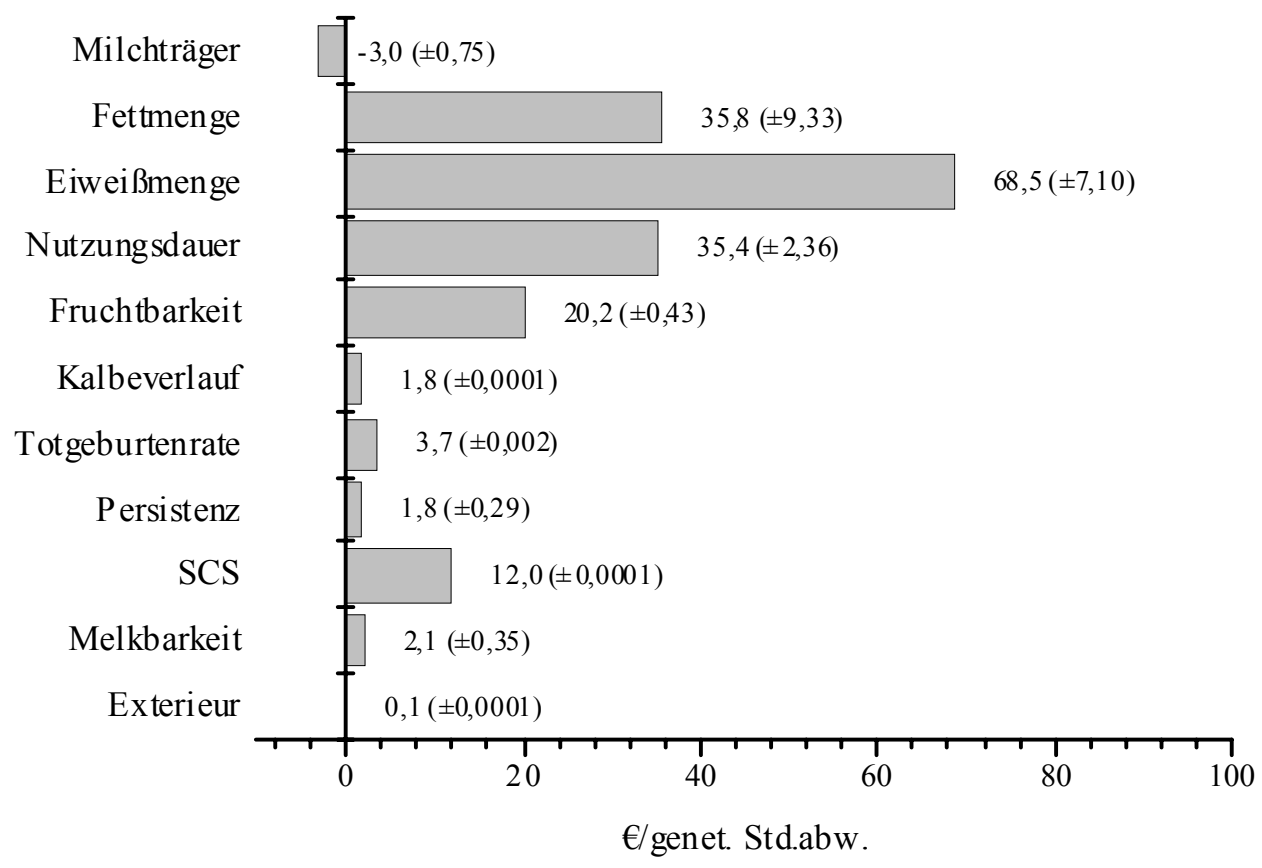

Abb. 4.3 Wirtschaftliche Gewichte ( \pm Standardabweichung) der einzelnen Merkmale in $€$ je genetischer Standardabweichung bei Holstein im Referenzszenario ohne Milchquotenbeschränkung

Dabei wird deutlich, dass das Merkmal Eiweißmenge mit etwa $69 € / \sigma_{\mathrm{a}}$ das höchste wirtschaftliche Gewicht aufweist und somit auch den größten Beitrag zur Wirtschaftlichkeit eines Betriebes leistet. Danach folgen die Merkmale Fettmenge und Nutzungsdauer mit etwa $35 € / \sigma_{a}$. Die Fruchtbarkeit weist ein wirtschaftliches Gewicht von $20 € / \sigma_{\mathrm{a}}$ und der SCS von $12 € / \sigma_{\mathrm{a}}$. Die restlichen Merkmale variieren zwischen $0,1 € / \sigma_{\mathrm{a}}$ und $2 € / \sigma_{\mathrm{a}}$. Das Merkmal Milchträger geht negativ in die Gewichtung ein, obwohl keine Beschränkung durch Milchquote vorliegt.

Wie schon bei den Grenznutzen der Merkmale für Holstein festgestellt werden kann, sind die Standardabweichungen bei den Milchleistungsmerkmalen am höchsten. Die Standardabweichungen der funktionalen Merkmale liegen in einem niedrigen Bereich, Es zeigt sich, dass der Einfluss des Milchauszahlungspreis und der Milchleistung auf diese Merkmale nur gering ist. 
In Abb. 4.4 sind die wirtschaftlichen Gewichte je genetischer Standardabweichung für das Referenzszenario mit und ohne Milchquotenbeschränkung im Vergleich dargestellt.

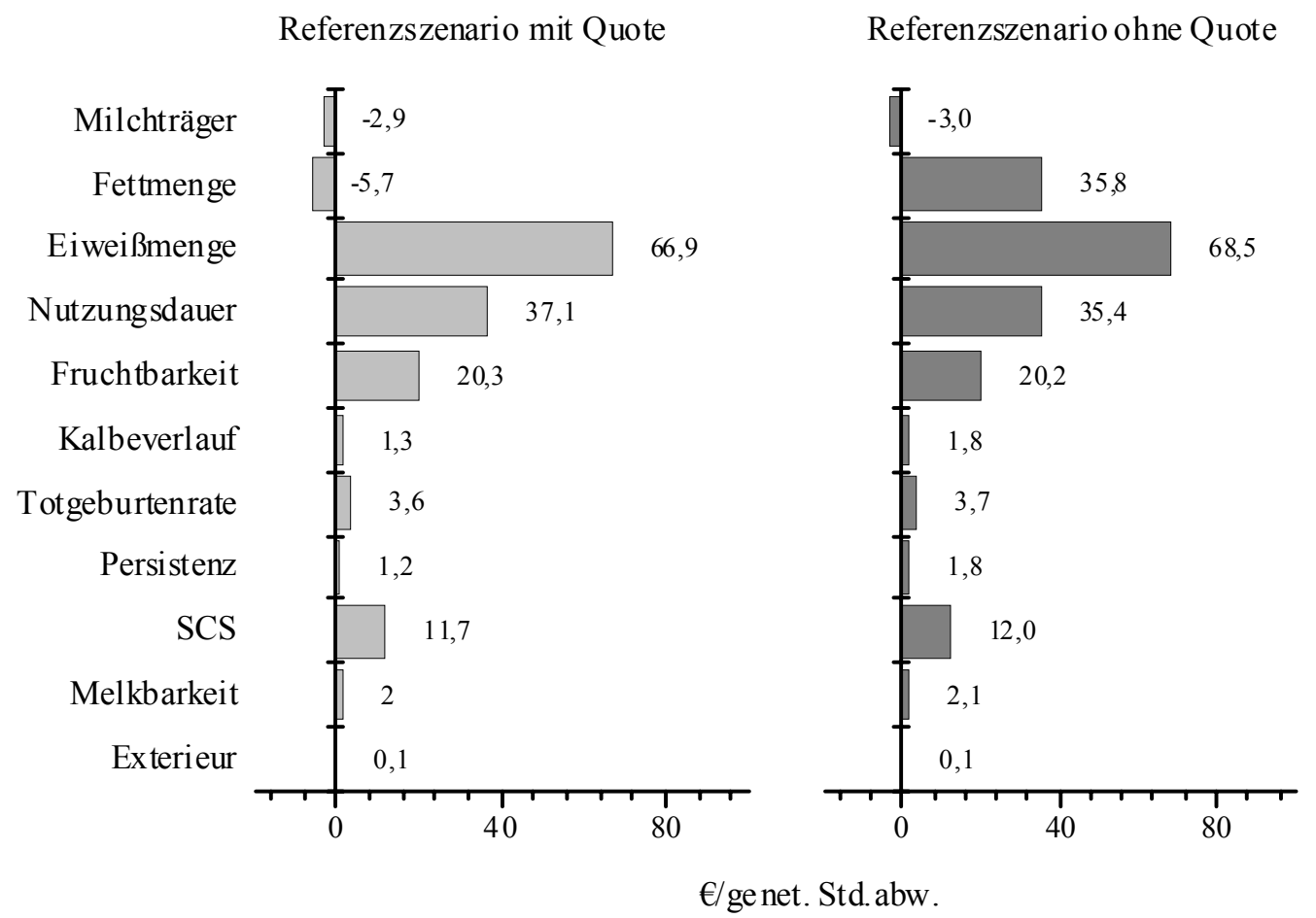

Abb. 4.4 Wirtschaftiche Gewichte der einzelnen Merkmale in $€$ je genetischer Standardabweichung bei Holstein ohne und mit Milchquotenbeschränkung im Vergleich

Es zeigt sich, dass im Referenzszenario mit Quote das wirtschaftliche Gewicht für die Fettmenge im negativen Bereich liegt (Abb. 4.4). Dies liegt an der unterstellten Milchquotenbegrenzung. Da die im Referenzszenario unterstellte Fettmenge in der Herde bei einer Steigerung dieses Merkmals die vorgegebene Quotenlimitierung übersteigt, wirkt sich dies negativ auf den Betriebsgewinn aus und das wirtschaftliche Gewicht sinkt ab. Das wirtschaftliche Gewicht für die Eiweißmenge ist unter Milchquotenbeschränkungen etwas geringer als ohne Quote. Auf die funktionalen Merkmale nimmt die Milchquote kaum Einfluss. 
Berechnet man die relativen wirtschaftlichen Gewichte, so hat der Milchleistungskomplex (Fett- und Eiweißmenge) einen Anteil von 58\% an den gesamten Merkmalen (Abb. 4.5).

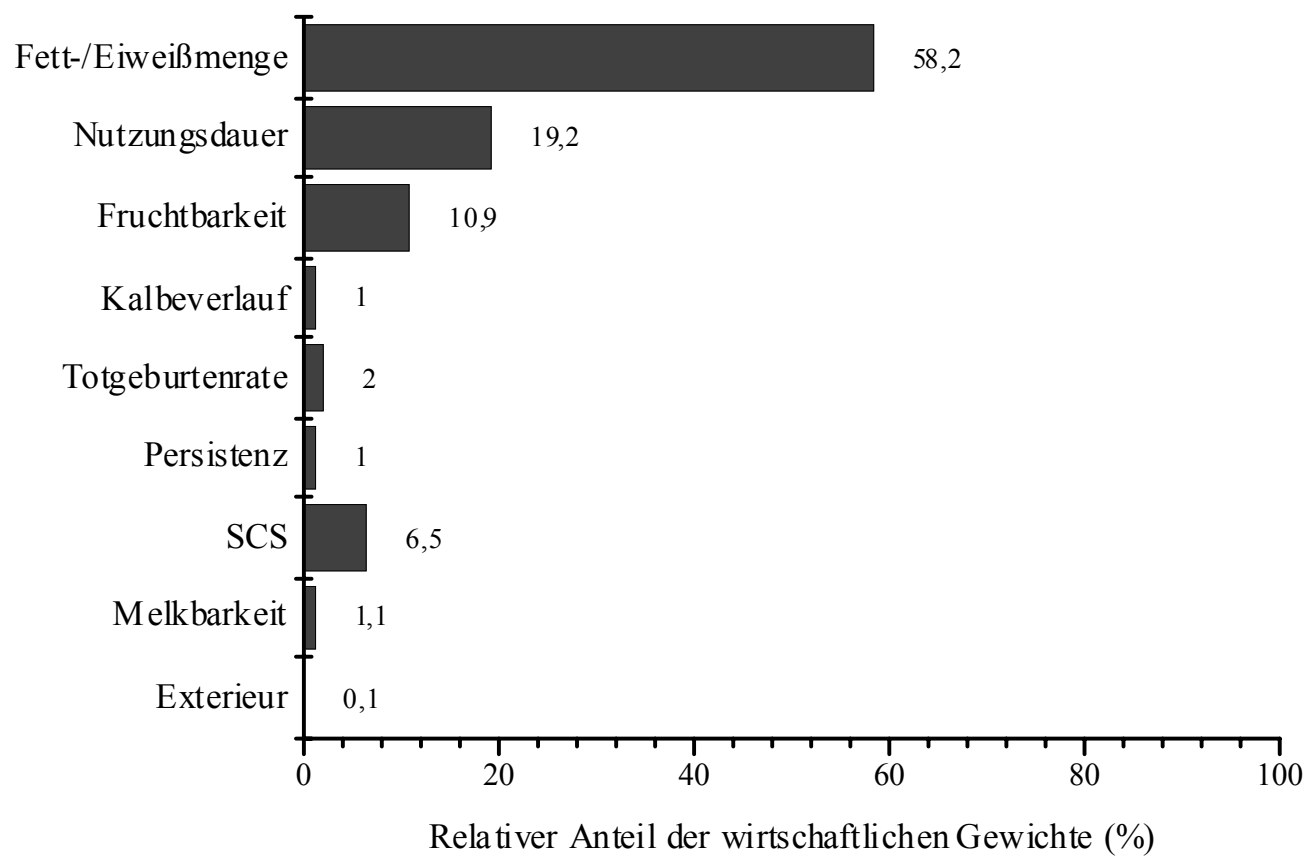

Abb. 4.5 Relative wirtschaftliche Gewichte der einzelnen Merkmale (\%) bei Holstein

Wie in Abb. 4.5 dargestellt, nehmen die Nutzungsdauer mit 19\% und der Komplex Zuchtleistung (Fruchtbarkeit, Kalbeverlauf und Totgeburtenrate) mit 14\% den zweitgrößten relativen Anteil ein. Der SCS weist ein relatives wirtschaftliches Gewicht von etwa 7\% auf, die Persistenz und Melkbarkeit jeweils 1\%. Das Exterieur hat den geringsten Anteil mit $0,1 \%$.

Neben den Kosten und Erlösen haben auch biologische Merkmale Einfluss auf die wirtschaftlichen Gewichte. Am Beispiel der durchschnittlichen Nutzungsdauer in der Herde sind die Auswirkungen auf die wirtschaftlichen Gewichte verschiedener Merkmale dargestellt (Abb. 4.6). 


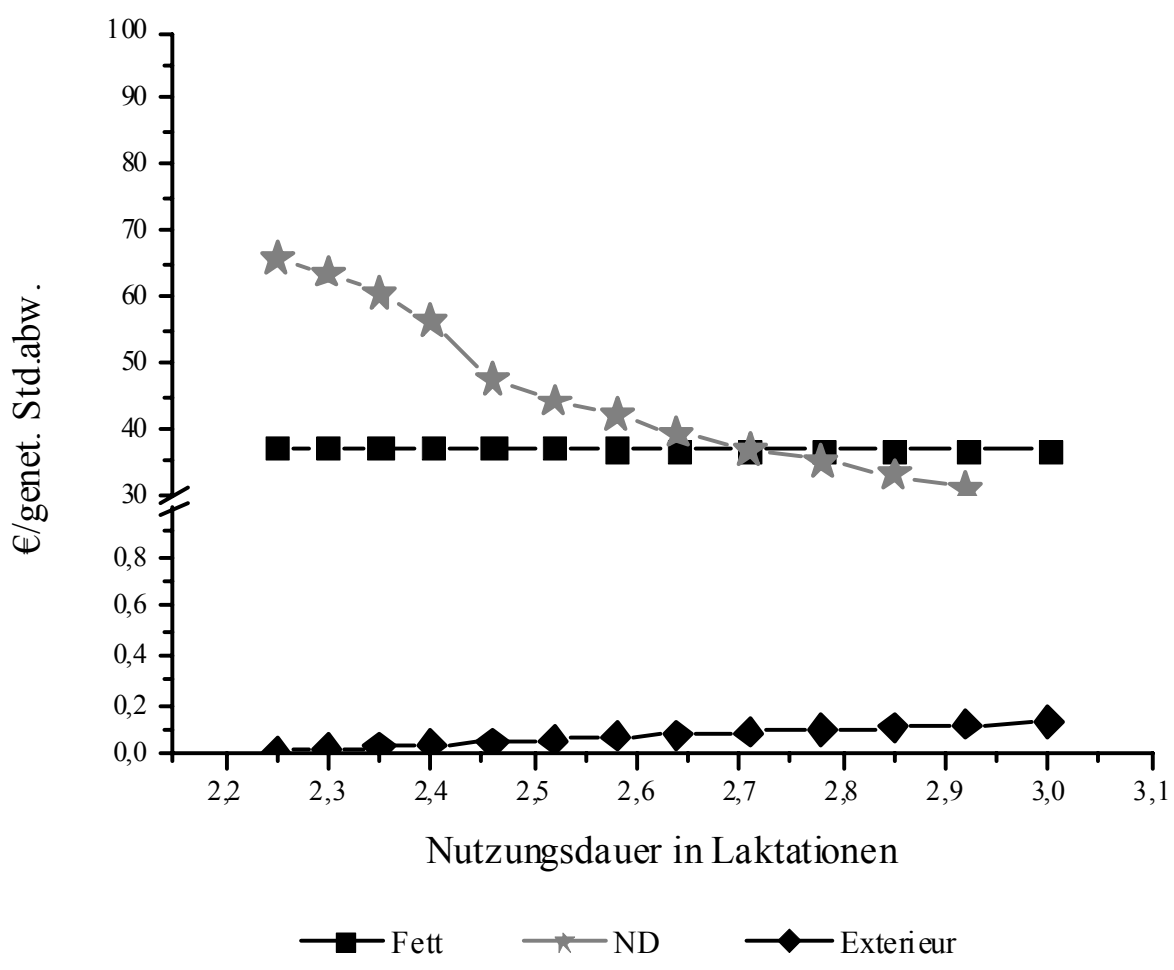

Abb.4.6 Einfluss der durchschnittlichen Nutzungsdauer auf die wirtschaftlichen Gewichte $\left(\epsilon / \sigma_{a}\right)$ der Merkmale Fettmenge (Fett), Nutzungsdauer (ND) und Exterieur (Exterieur) bei Holstein

Wird die durchschnittliche Nutzungsdauer in der Milchviehherde variiert, zeigt dies keinen Einfluss auf das wirtschaftliche Gewicht der Fettmenge (Abb. 4.6). Hingegen wird das wirtschaftliche Gewicht der Nutzungsdauer mit steigender durchschnittlicher Nutzungsdauer geringer. Bei der Verbesserung der Nutzungsdauer verbleiben die Kühe länger in der Herde, die Remontierungskosten werden gesenkt und mehr Tiere erreichen das letzte Drittel der Laktation. Hat die Herde schon eine hohe durchschnittliche Nutzungsdauer, so bewirkt eine weitere Erhöhung weniger Zusatznutzen, als dies auf einem niedrigen Niveau der Fall wäre. Es tritt bei diesem Merkmal der Fall des abnehmenden Grenznutzens ein. Das Exterieur hat bei steigender durchschnittlicher Nutzungsdauer in der Herde einen stetig ansteigenden Nutzen. Verbleiben mehr Kühe in der Herde, so stehen mehr Färsen zum Zuchtviehverkauf zur Verfügung. Die Kosten für die Remontierung können besser auf die gesamte Herde und den Verbleib in der Herde verteilt werden und das wirtschaftliche Gewicht des Exterieurs steigt kontinuierlich. 
Stellt man nun, wie in Abb. 4.7, die Gewichtung der berechneten Merkmalskomplexe denen der derzeitigen Zuchtwertschätzung (VIT, 2006) gegenüber, zeigt sich eine deutliche Veränderung in der Zuordnung.

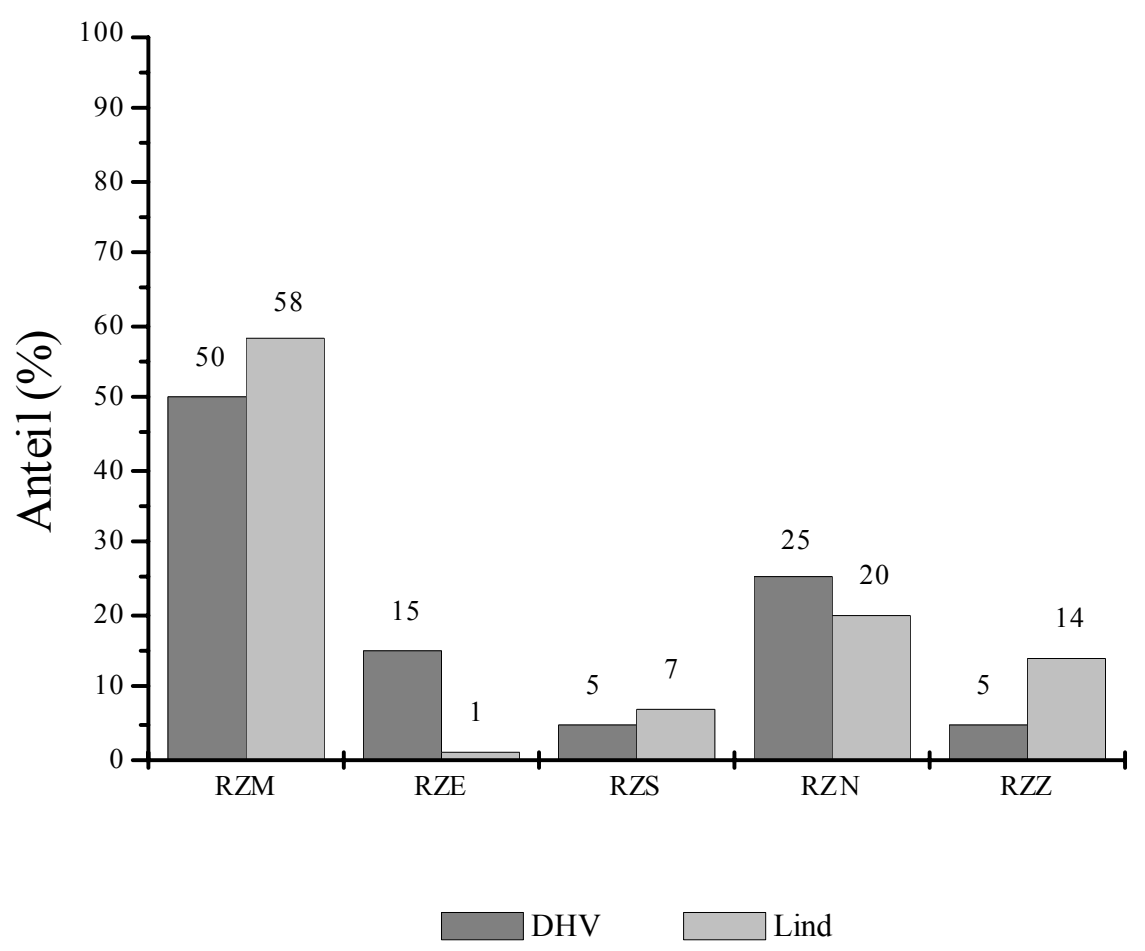

Abb. 4.7 Vergleich der relativen wirtschaftlichen Gewichte (\%) der eigenen Berechnungen mit der derzeitigen Gewichtung der Teilzuchtwerte nach DHV bei Holstein (VIT, 2006)

Der Anteil des RZM erhöht sich von 50\% auf 58\%, der des RZE verringert sich deutlich von 15\% auf 1\%. Ebenfalls geringer ist der Anteil des RZN, der sich von 25\% auf $20 \%$ reduziert, wohingegen sich der RZS und der RZZ erhöhen. In der neuen Verteilung der Merkmalskomplexe zeigt sich, dass die Milchleistungsmerkmale weiterhin bedeutend für die Wirtschaftlichkeit und damit auch die Zucht sind, das Exterieur unter wirtschaftlichen Gesichtspunkten der hohen Gewichtung nicht standhalten kann und die Zuchtleistung und dementsprechend die Fruchtbarkeit an Bedeutung gewinnt. 


\section{RZG-Varianten}

Eine deutliche Veränderung der wirtschaftlichen Gewichtung im RZG kommt den Exterieurmerkmalen und damit dem RZE zu. Da in verschiedensten Arbeiten (FürstWaltl et al., 2004; Krogmeier et al., 2006; Schierenbeck, 2006) auf die Bedeutung der Exterieurmerkmale für die Züchter hingewiesen wird, soll mit einem Ansatz nach Schierenbeck (2006) der Anteil der Exterieurmerkmale nochmals untersucht werden.

Wird der zusätzliche Nutzen eines Euter- bzw. Fundamentpunktes unterstellt (Schierenbeck, 2006), so ergibt sich ein zusätzlicher Gewinn von 17,91€ je verbesserter Euternote und 11,25€ je verbesserter Fundamentnote. Setzt man dieses als Grenznutzen für die Exterieurmerkmale ein, erhalten diese einen Anteil an 9\% bzw. $6 \%$ an allen Merkmalen (Tab. 4.2).

Tab. 4.2 Varianten der Gewichtung des Gesamtzuchtwertes bei Veränderung des relativen wirtschaftlichen Gewichtes (\%) für die Exterieurmerkmale bei Holstein (VIT, 2006)

\begin{tabular}{lcccc}
\hline & DHV & $1 \%$ & $6 \%$ & $9 \%$ \\
\hline RZM & 50 & 58 & 56 & 54 \\
RZE & 15 & 1 & 6 & 9 \\
RZS & 5 & 7 & 6 & 6 \\
RZN & 25 & 20 & 19 & 18 \\
RZZ & 5 & 14 & 13 & 13 \\
\hline
\end{tabular}

Setzt man die jeweiligen Anteile des Exterieurs wie vorgeschlagen um, so sind die einzelnen Varianten des Gesamtzuchtwertes wie in Tab. 4.2 dargestellt. Der RZE gewinnt an Gewichtung während RZM und RZN etwas zurückgehen.

Um den Einfluss der einzelnen Varianten des Gesamtzuchtwertes auf die Zuchtwerte der Holsteinbullen darzustellen, ist die Rangierung ausgewählter Bullen in den jeweiligen Varianten in Abb. 4.8 aufgezeigt. 


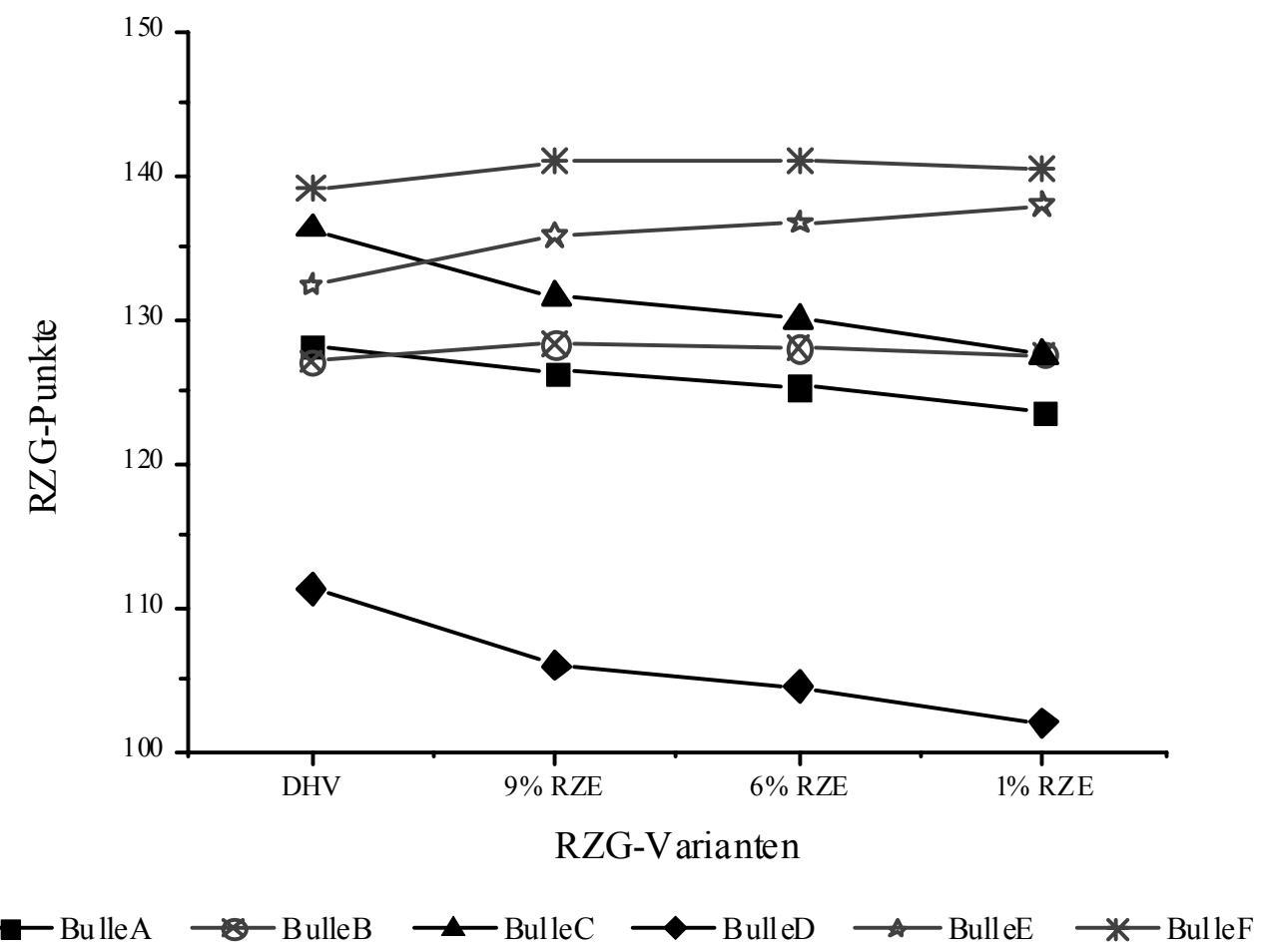

Abb. 4.8 Rangierung ausgewählter Bullen nach RZG (Zuchtwertpunkte) bei unterschiedlichen Anteilen des RZE bei Holstein

Die Bullen A, C und D weisen einen hohen RZE bei gleichzeitig ausgeglichenen RZM, RZS, RZN und RZZ auf. Im Gegensatz dazu haben die Bullen B, E und F einen hohen RZM und hohen RZZ, aber durchschnittlichen RZE. Es zeigt sich, dass die Bullen A, C und D mit der neuen Variante und den abgestuften Anteilen des RZE an RZG verlieren. Dies lässt sich dadurch erklären, dass alle drei Bullen einen hohen RZE über 130 Punkte haben und durch den verminderten Anteil der RZG zurückgeht. Der RZG der Bullen B, E und F steigt hingegen an, da deren RZE nur leicht überdurchschnittlich ist, sie aber durch einen RZM über 125 Punkte und einem RZZ über 112 Punkte und deren höheren Gewichtung im RZG einen verbesserten Gesamtzuchtwert aufweisen.

Aufgrund dieser Untersuchung zeigt sich, dass unter Annahme der neuen Gewichtung einige Veränderungen in der derzeitigen Zuchtwertschätzung auftreten. Bullen mit einem überdurchschnittlich hohen Zuchtwert für Exterieur fallen im Gesamtzuchtwert etwas zurück, Bullen mit gutem RZZ hingegen gewinnen an RZGPunkten. 
Anhand der Top-100-Bullen in der derzeitigen Zuchtwertschätzung sind Mittelwerte über deren jeweilige Relativzuchtwerte unter den jeweiligen Gesamtzuchtwertvarianten berechnet worden und in Abb. 4.9 dargestellt.

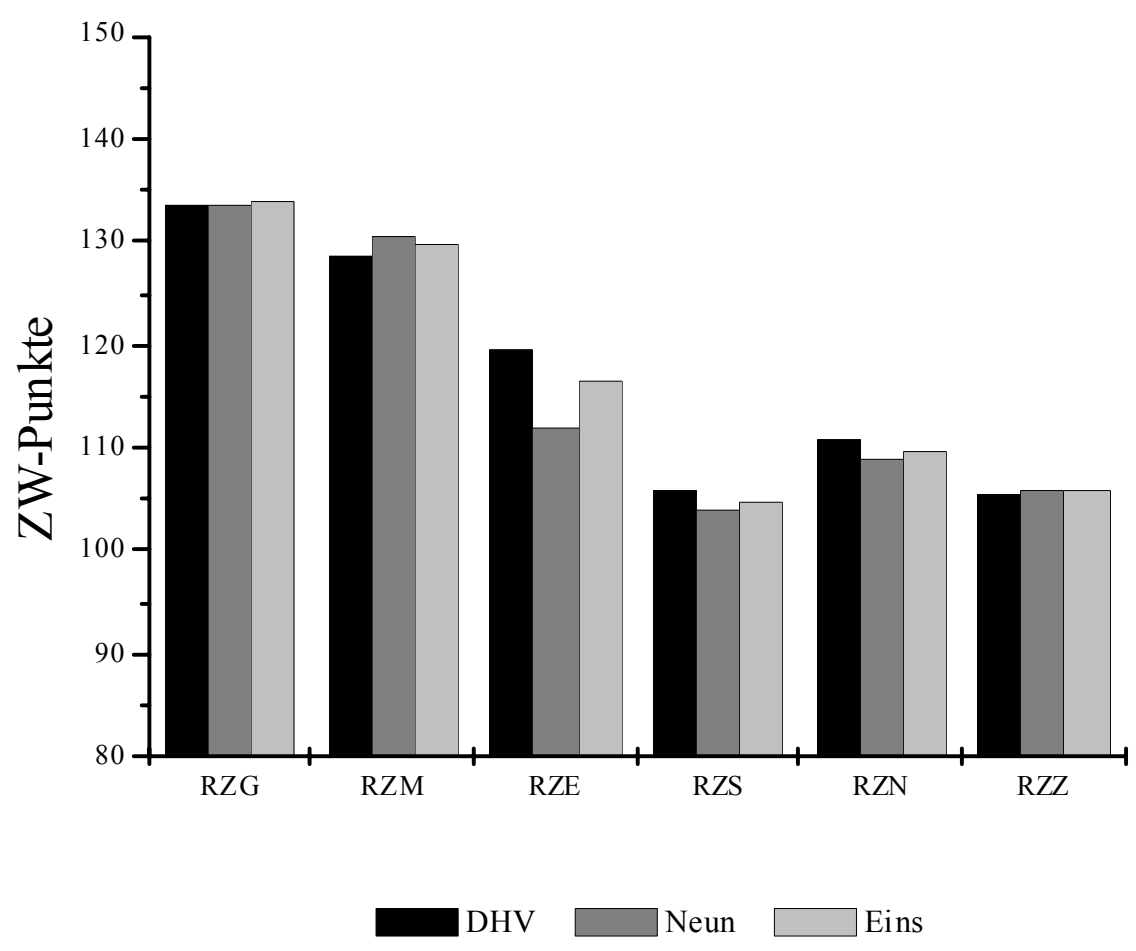

Abb. 4.9 Mittelwerte der Relativzuchtwerte der Top-100-Bullen in den drei RZGVarianten (DHV, neu mit 9\% und neu mit 1\% Gewichtung des RZE)

Es zeigt sich, dass eine neue Zusammensetzung des Gesamtzuchtwertes keine negativen Auswirkungen auf die Höhe des RZG unter den ersten 100 Bullen der Zuchtwertliste hat (Abb. 4.9). Dabei sind in jeder der berechneten RZG-Variante unterschiedliche Bullen in der Top-100-Liste, da sich die Zuchtwerte der Bullen in den einzelnen Varianten neu formieren. Im Mittel haben die Top-100-Bullen sogar einen leicht höheren RZG in der RZG-Variante mit einem Anteil von 9\% RZE gegenüber der derzeitigen Gesamtzuchtwert-Zusammensetzung bei Holstein. Zu einer Erhöhung der Mittelwerte kommt es beim RZM und RZZ, dagegen gehen die Mittelwerte beim RZE, RZS und RZN zurück. Zusätzlich ist zu berücksichtigen, dass die Relativzuchtwerte neben den wirtschaftlichen Gewichten auch durch die genetischen Beziehungen untereinander und die Heritabilitäten der einzelnen Merkmale beeinflusst werden. 


\subsubsection{Fleckvieh}

Aus dem Referenzszenario heraus werden Grenznutzen bzw. wirtschaftliche Gewichte für die Rasse Fleckvieh abgeleitet. Alternativ zur Ableitung wirtschaftlicher Gewichte im Referenzszenario werden bei Fleckvieh zusätzlich zwei Vergleichsszenarien definiert.

Im Referenzszenario wird ein zukünftiger Milchauszahlungspreis angenommen, indem eine größtmögliche Spreizung des Fett- und Eiweißpreises vorgenommen wird. Im Vergleichsszenario wird der aktuelle Milchauszahlungspreis in Bayern (Erzeugerorientierungspreis) unterstellt und die Gewichte werden sowohl ohne als auch mit Milchquotenbeschränkungen berechnet.

In Tab. 4.3 sind die genetischen Standardabweichungen der einzelnen Merkmale bei Fleckvieh aufgelistet.

Tab. 4.3 Genetische Standardabweichung der einzelnen Merkmale bei Fleckvieh (Dodenhoff, 2005)

\begin{tabular}{lcc}
\hline & Merkmalseinheit & Genetische Standardabweichung \\
\hline Milchträger & $\mathrm{kg}$ & 570 \\
Fettmenge & $\mathrm{kg}$ & 21,9 \\
Eiweißmenge & $\mathrm{kg}$ & 16,4 \\
Nutzungsdauer & Tage & 180 \\
Fruchtbarkeit & $\%$ & 4,5 \\
Totgeburtenrate & Klasse & 4,0 \\
Kalbeverlauf & $\sigma$ & 0,22 \\
Persistenz & Klasse & 2,5 \\
SCS & $\mathrm{kg} / \mathrm{Min}$ & 0,5 \\
Melkbarkeit & $\mathrm{g}$ & 0,29 \\
Nettozunahme & Klasse & 26,5 \\
Handelsklasse & $\%$ & 0,25 \\
Ausschlachtung & & 1,15 \\
\hline
\end{tabular}


Zunächst sind in Abb. 4.10 die Grenznutzen je Merkmalseinheit sowie die jeweiligen Standardabweichungen der Grenznutzen bei Fleckvieh im Referenzszenario dargestellt. Das Referenzszenario beinhaltet die vorgestellten Parameter und ist bei der Berechnung des Milchpreises durch einen gespreizten Fett- und Eiweißpreis gekennzeichnet. Zusätzlich unterliegt die Produktion keinen Quotenbeschränkungen.

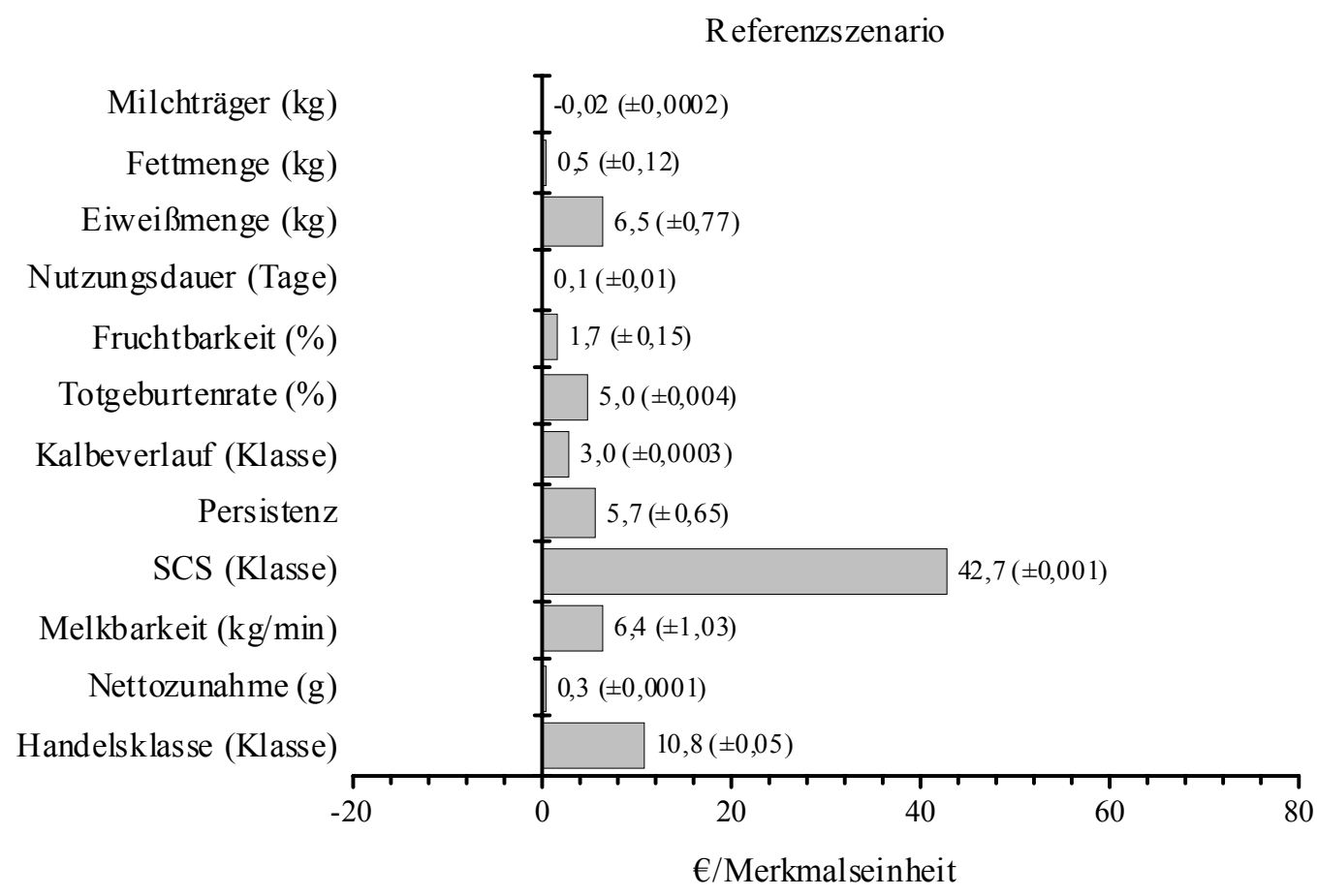

Abb. 4.10 Grenznutzen ( \pm Standardabweichung) der einzelnen Merkmale in $€$ je Merkmalseinheit bei Fleckvieh im Referenzszenario

Der Grenznutzen des Milchträgers ist negativ, die Fettmenge weist eine Grenznutzen von $0,5 €$ je $\mathrm{kg}$ verbesserter Fettmenge auf, die Eiweißmenge einen Grenznutzen von $6,5 €$ je kg verbesserter Eiweißmenge (Abb. 4.10). Wird die Nutzungsdauer erhöht, so ergibt sich ein zusätzlicher Nutzen von etwa $3 €$ je Monat. Der Grenznutzen der funktionalen Merkmale liegt zwischen $1 €$ und etwa $6 €$ je verbesserter Merkmalseinheit. Lediglich der SCS zeigt einen hohen Grenznutzen bei einer Verbesserung um eine Klasse.

In Abb. 4.11 sind die wirtschaftlichen Gewichte der einzelnen Merkmale in $€$ je genetischer Standardabweichung im Referenzszenario abgebildet. 


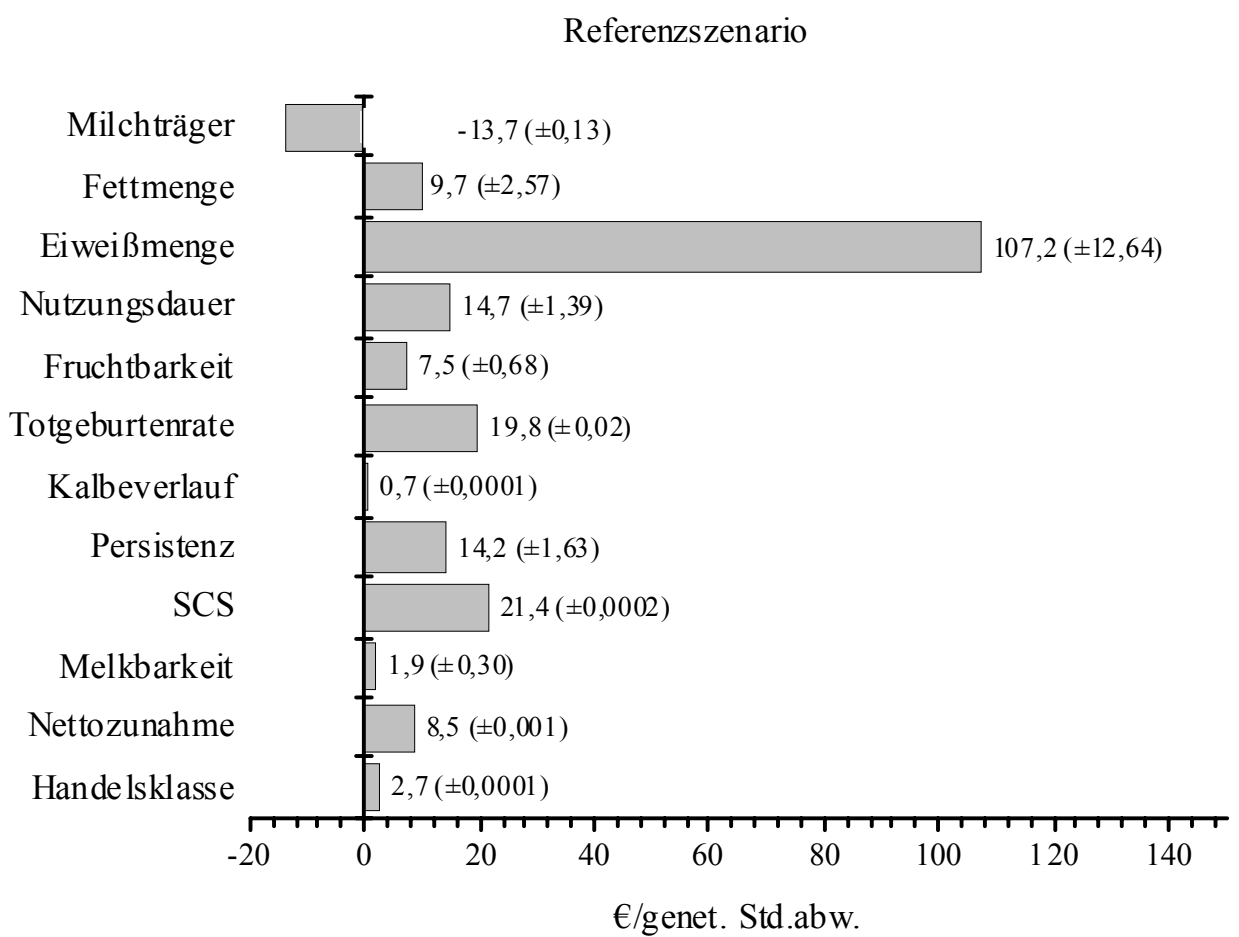

Abb. 4.11 Wirtschaftliche Gewichte ( \pm Standardabweichung) der einzelnen Merkmale in $€$ je genetischer Standardabweichung im Referenzszenario bei Fleckvieh

Das höchste wirtschaftliche Gewicht hat mit $107 € / \sigma_{\mathrm{a}}$ die Eiweißmenge (Abb. 4.11). Die beiden anderen Milchleistungsmerkmale Fettmenge und Milchträger liegen beide knapp unter bzw. über $10 € / \sigma_{\mathrm{a}}$, wobei das wirtschaftliche Gewicht des Milchträgers negativ ist. Eine Erhöhung dieses Merkmals führt zu einer Verminderung des Gewinnes im Betrieb. Bei den funktionalen Merkmalen haben der SCS $\left(21 € / \sigma_{a}\right)$, die Totgeburtenrate $\left(20 € / \sigma_{a}\right)$ und die Nutzungsdauer $\left(15 € / \sigma_{a}\right)$ die höchste Gewichtung. Die Persistenz ist mit $14 € / \sigma_{\mathrm{a}}$ ebenfalls hoch, während das Gewicht der Fruchtbarkeit bei $8 € / \sigma_{a}$ liegt und Kalbeverlauf und die Melkbarkeit ein geringes wirtschaftliches Gewicht aufweisen. Die Fleischleistungsmerkmale liegen generell auf einem niedrigen Niveau. Die Nettozunahme hat mit $9 € / \sigma_{\mathrm{a}}$ den höchsten Anteil der Merkmale, das Gewicht der Handelsklasse liegt bei $3 € / \sigma_{a}$.

Die Standardabweichungen der wirtschaftlichen Gewichte bei Fleckvieh verhalten sich ähnlich wie bei der Rasse Holstein. Auch hier sind die Standardabweichungen der Milchleistungsmerkmale aufgrund der Variation im Milchpreis und in der Milch- 
leistung am höchsten. Bei den funktionalen Merkmalen und den Fleischleistungsmerkmalen sind die Standardabweichungen klein, da diese Merkmale in den Berechnungen nicht variiert werden.

Das Referenzszenario wird eindeutig von den Milchleistungsmerkmalen, speziell der Eiweißmenge, dominiert. Dies ist auf den hohen unterstellten Auszahlungspreis von $6,70 €$ je kg Eiweiß zurückzuführen. Dementsprechend wirtschaftlich lohnend ist eine Steigerung der Eiweißmenge im Betrieb. Durch den geringen Auszahlungspreis der Fettmenge ( $1 €$ je kg Fett) liegt der zusätzliche Gewinn bei Steigerung dieses Merkmals um eine genetische Standardabweichung nur im Bereich von 10€. Der Milchträger bekommt das negative wirtschaftliche Gewicht, da dieses Merkmal keine Bezahlung erhält. Daher kommt es zu einer Verminderung des Gewinnes, da durch eine Steigerung des Milchträgers zwar die Menge erhöht wird, allerdings die Inhaltsstoffe verdünnt werden. Das wirtschaftliche Gewicht der Totgeburtenrate liegt auf einem sehr hohen Niveau. Allerdings ist dies in den Annahmen des Referenzszenarios begründet. Die Verkaufspreise für Kälber und Verkaufs- und Schlachtpreise der Kühe und Färsen sind sehr hoch angesetzt. Ebenso ist die durchschnittliche Nutzungsdauer der Herde bei nahezu drei Laktationen. Wird nun die Totgeburtenrate verbessert, kann man beim Verkauf eines Kalbes bzw. zu einem späteren Zeitpunkt als Färse oder Kuh einen besseren Erlös bei gleich bleibenden Kosten erzielen. Der Zusatznutzen ist bei hohen Verkaufpreisen höher als bei geringen Verkaufspreisen.

Im Vergleichsszenario (Abb. 4.12) wird anstelle eines gespreizten Auszahlungspreises für Fett und Eiweiß der Erzeugerorientierungspreis in Bayern unterstellt, der den derzeitigen Milchauszahlungspreis widerspiegelt. Die Annahmen sind die gleichen wie im Referenzszenario, nur der Milchpreisauszahlungspreis ist verändert, um die Auswirkungen unterschiedlicher Preisannahmen untersuchen zu können. Die Berechnungen erfolgen ohne Milchmengenbegrenzung. 
Vergleichsszenario ohne Quote

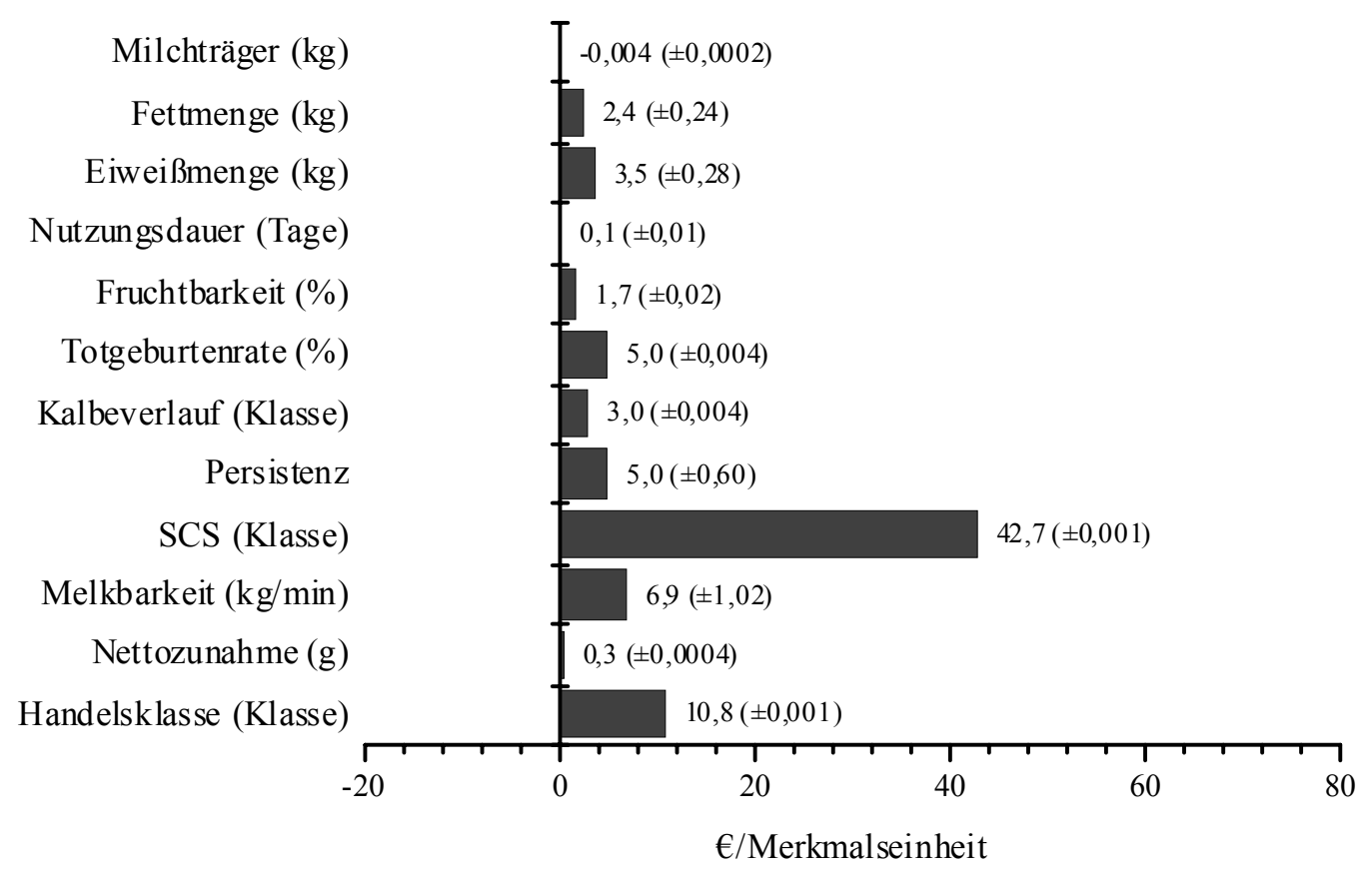

Abb. 4.12 Grenznutzen ( \pm Standardabweichung) der einzelnen Merkmale in $€$ je Merkmalseinheit bei Fleckvieh im Vergleichsszenario ohne Milchquotenbeschränkungen

Es zeigt sich, dass das Verhältnis der Grenznutzen von Fett- zu Eiweißmenge im Gegensatz zum Referenzszenario enger wird (Abb. 4.12). Die Grenznutzen der funktionalen Merkmale und der Fleischleistungsmerkmale verändern sich nur geringfügig.

Die wirtschaftlichen Gewichte der einzelnen Merkmale der Rasse Fleckvieh im Vergleichsszenario ohne Quote sind in Abb. 4.13 aufgeführt. 


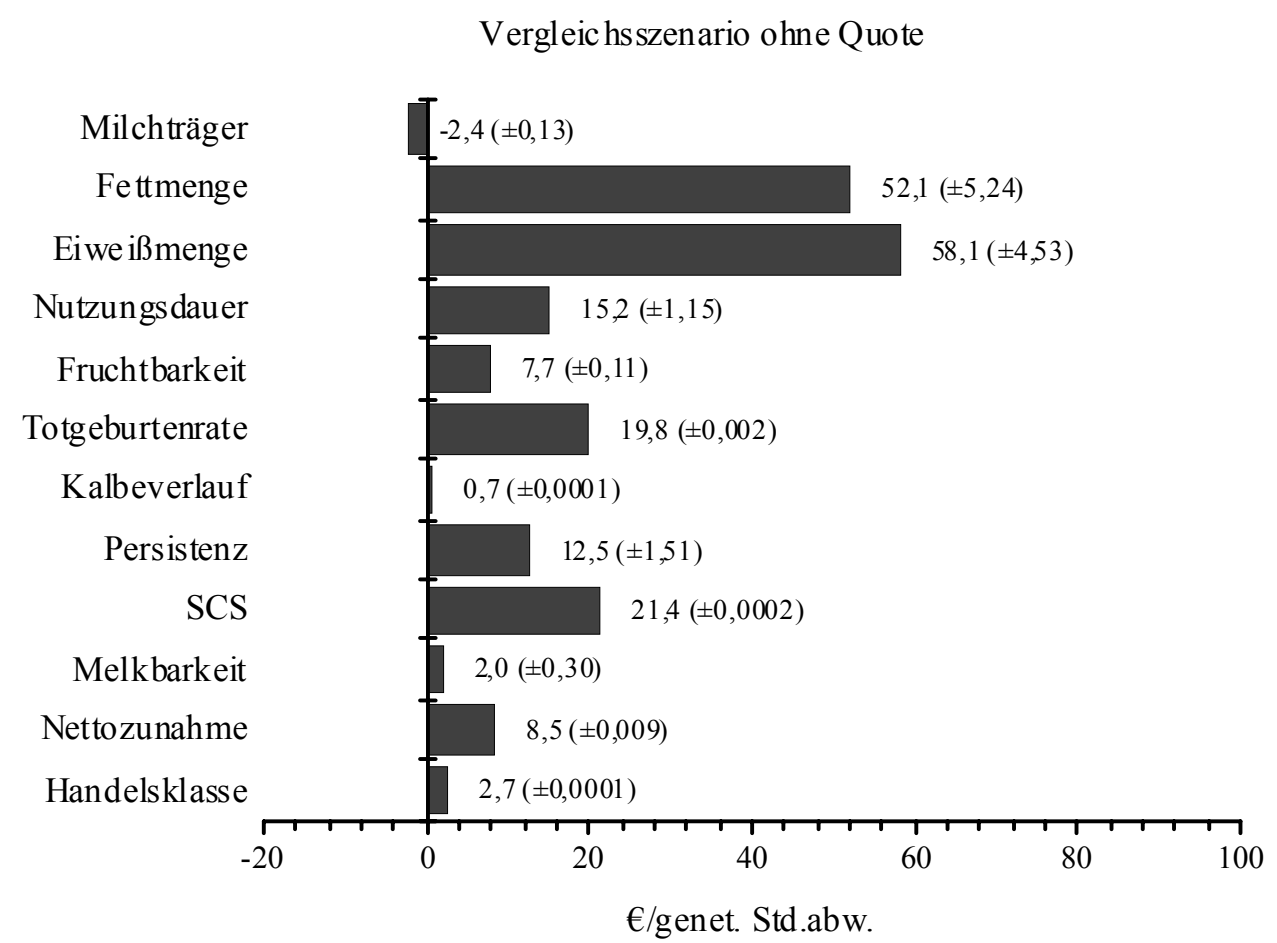

Abb. 4.13 Wirtschaftliche Gewichte ( \pm Standardabweichung) der einzelnen Merkmale in $€$ je genetischer Standardabweichung im Vergleichsszenario ohne Milchquotenbeschränkung bei Fleckvieh

Die größten Veränderungen der wirtschaftlichen Gewichte liegen beim Vergleichsszenario ohne Quotenbeschränkungen im Vergleich zum Referenzszenario bei den Milchleistungsmerkmalen vor (Abb. 4.13). Das wirtschaftliche Gewicht für die Eiweißmenge reduziert sich auf die Hälfte $\left(58 € / \sigma_{a}\right)$, das Gewicht der Fettmenge hingegen steigt auf $52 € / \sigma_{\mathrm{a}}$ an und das des Milchträgers steigt auf $-2 € / \sigma_{\mathrm{a}}$. Der Auszahlungspreis für Milchträger, Fett- und Eiweißmenge ist die Ursache für diese Gewichtung. Allerdings haben die Milchleistungsmerkmale in Relation gesehen immer noch den größten relativen Anteil an den Gewichten. Die wirtschaftlichen Gewichte für SCS, Totgeburtenrate, Fruchtbarkeit, Melkbarkeit, Nettozunahme und Handelsklasse bleiben in etwa auf dem gleichen Niveau wie im Referenzszenario, die der Nutzungsdauer und Persistenz gehen leicht zurück. Der Einfluss des Bezahlungspreises bezieht sich nicht ausschließlich auf die Milchleistungsmerkmale, sondern auch auf die mit der Milchleistung verbunden Merkmale wie Nutzungsdauer und Persistenz. 
In Abb. 4.14 sind die Szenarien nochmals nebeneinander gestellt. Zusätzlich zu den zwei vorgestellten Szenarien ist als Alternative das Vergleichsszenario unter Quotenbedingungen aufgeführt. Dies würde die aktuelle Situation in Deutschland mit dem derzeitigen Milchauszahlungspreis und Milchquotenbeschränkungen widerspiegeln.

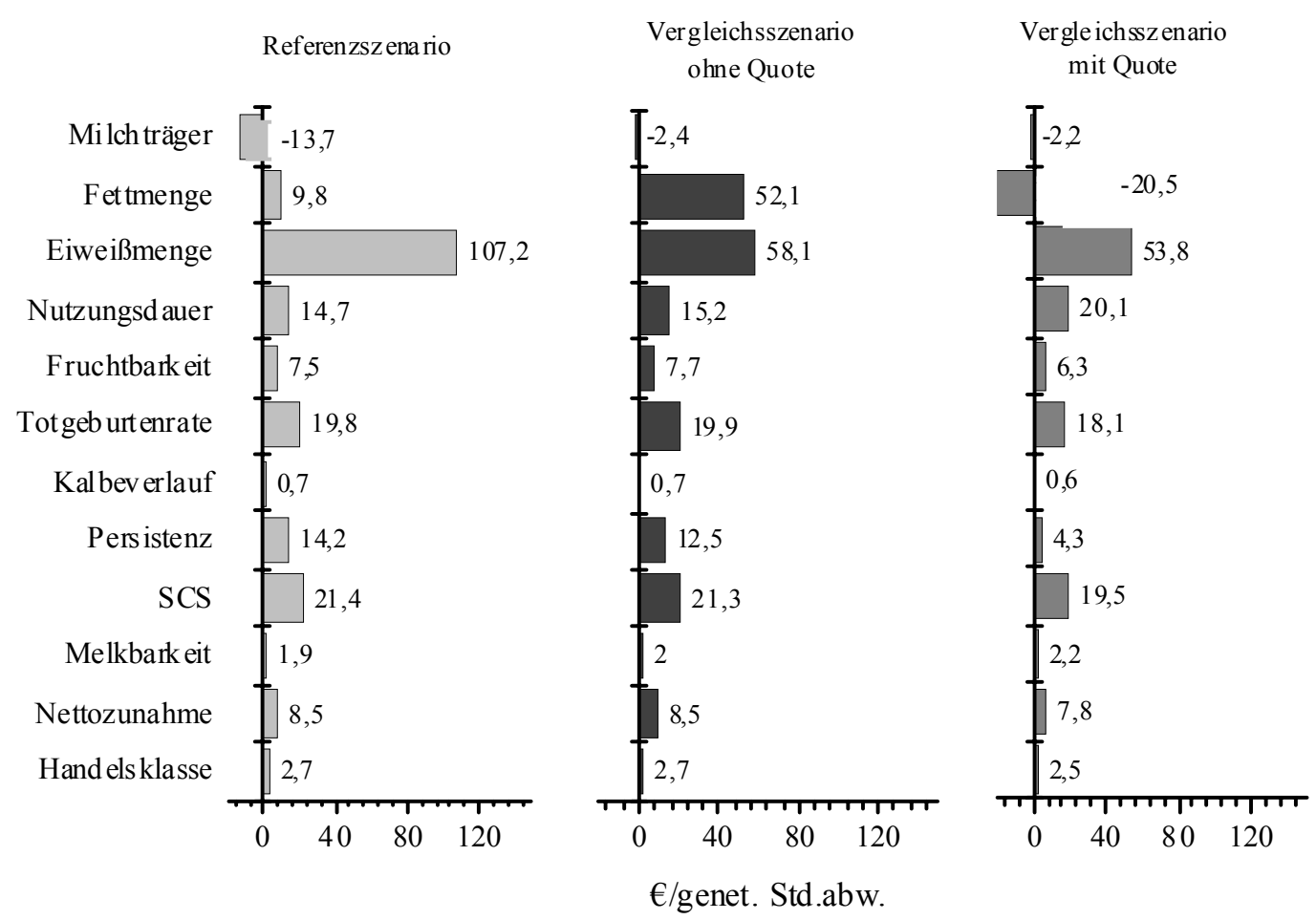

Abb. 4.14 Wirtschaftiche Gewichte der einzelnen Merkmale in $€$ je genetischer Standardabweichung bei Fleckvieh im Vergleich der Szanarien

Vergleicht man die drei Szenarien, so fällt auf, dass die größten Unterschiede in den wirtschaftlichen Gewichten der Milchleistungsmerkmale vorliegen (Abb. 4.14). Unter Milchquotenbeschränkung (Vergleichsszenario mit Quote) wird zusätzlich das wirtschaftliche Gewicht der Fettmenge negativ. Dies ist auf die unterstellte Milchquote mit Referenzfettgehalt zurückzuführen. Wird unter Quotensituation die Fettmenge in der Herde erhöht, so ist die Quote mit der gleichen Anzahl Kühe bereits früher erfüllt. Der daraus resultierende Gewinn des Betriebes ist geringer als vor der Merkmalsverbesserung, was zu einem negativen Grenznutzen für dieses Merkmal führt. Mit der Quotensituation steigt auch das wirtschaftliche Gewicht für Nutzungsdauer an. Durch die Quotenbedingungen ist die Milchmenge in der Herde begrenzt. Durch eine Erhöhung der Nutzungsdauer kann so mehr Milch zu geringeren Kosten 
produziert werden. Dies ist unter Quotenbedingungen rentabler als ohne Milchquotenbegrenzungen.

In der folgenden Abbildung (Abb. 4.15) sind die einzelnen Merkmalskomplexe zusammengefasst und im jeweiligen Szenario dargestellt.

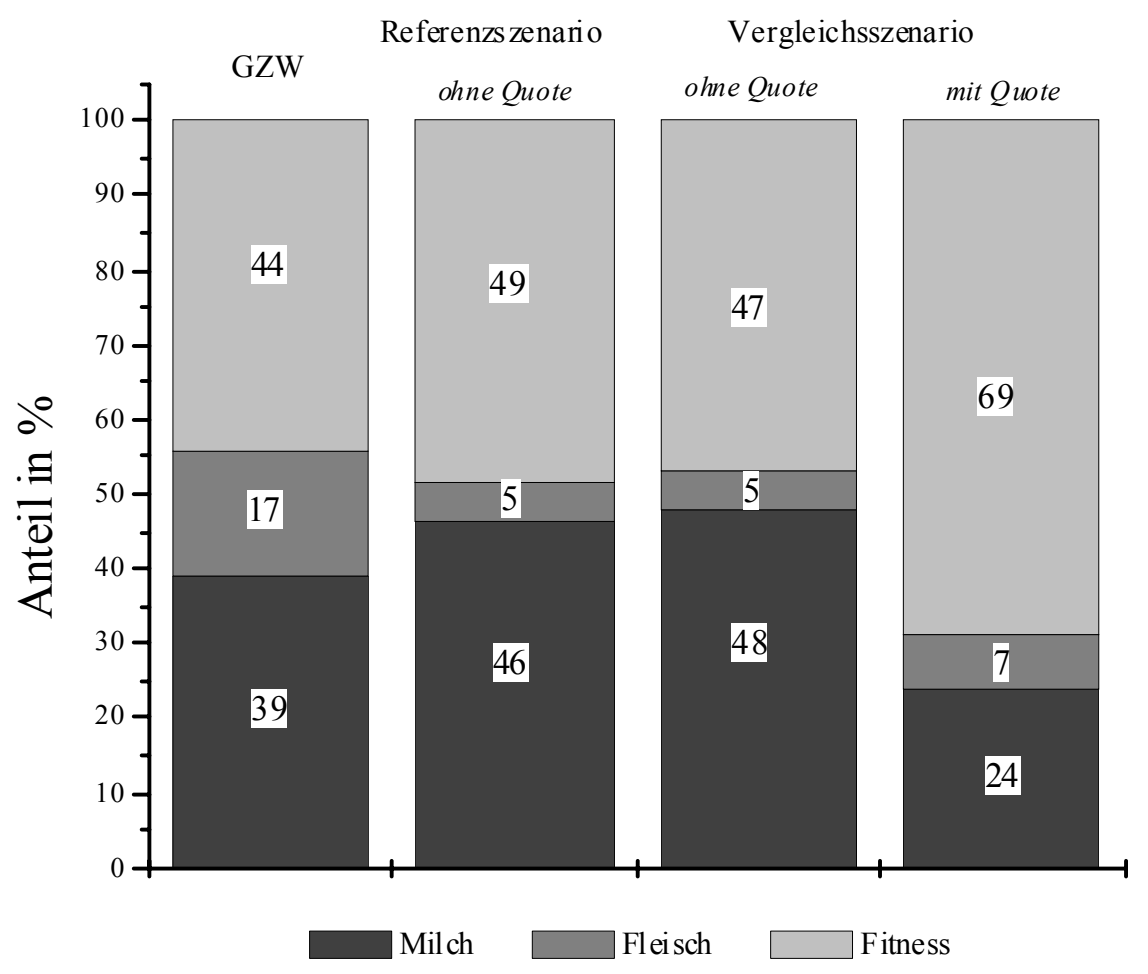

Abb.4.15 Anteil der einzelnen Merkmalskomplexe (\%) beim Gesamtzuchtwert (GZW) für Fleckvieh 2006 (Miesenberger, 1999) und den drei vorgestellten Szenarien

Das Szenario GZW zeigt die Verteilung der Merkmalskomplexe bei Fleckvieh in 2006 (Abb. 4.15). Der Komplex Milch hat einen Anteil von 39\%, Fleisch 17\% und die Fitnessmerkmale 44\%. Betrachtet man das Referenzszenario, so wird deutlich, dass sowohl der Merkmalskomplex Milch als auch Fitness zunimmt, der Komplex Fleischleistung aber an Gewichtung verliert. Im Vergleichszenario ohne Quote gewinnen die Milchleistungsmerkmale auf Kosten der Fitnessmerkmale noch zusätzlich an Gewicht. Berechnet man die wirtschaftlichen Gewichte im Vergleichsszenario mit Quote geht der Einfluss der Milchleistungsmerkmale zurück, während die Fit- 
nessmerkmale an Bedeutung zunehmen. Die Fleischleistungsmerkmale behalten in etwa ihren Einfluss.

Die Auswirkungen eines veränderten Schlachtpreises für Kühe auf die wirtschaftlichen Gewichte sind in Abb. 4.16 dargestellt.

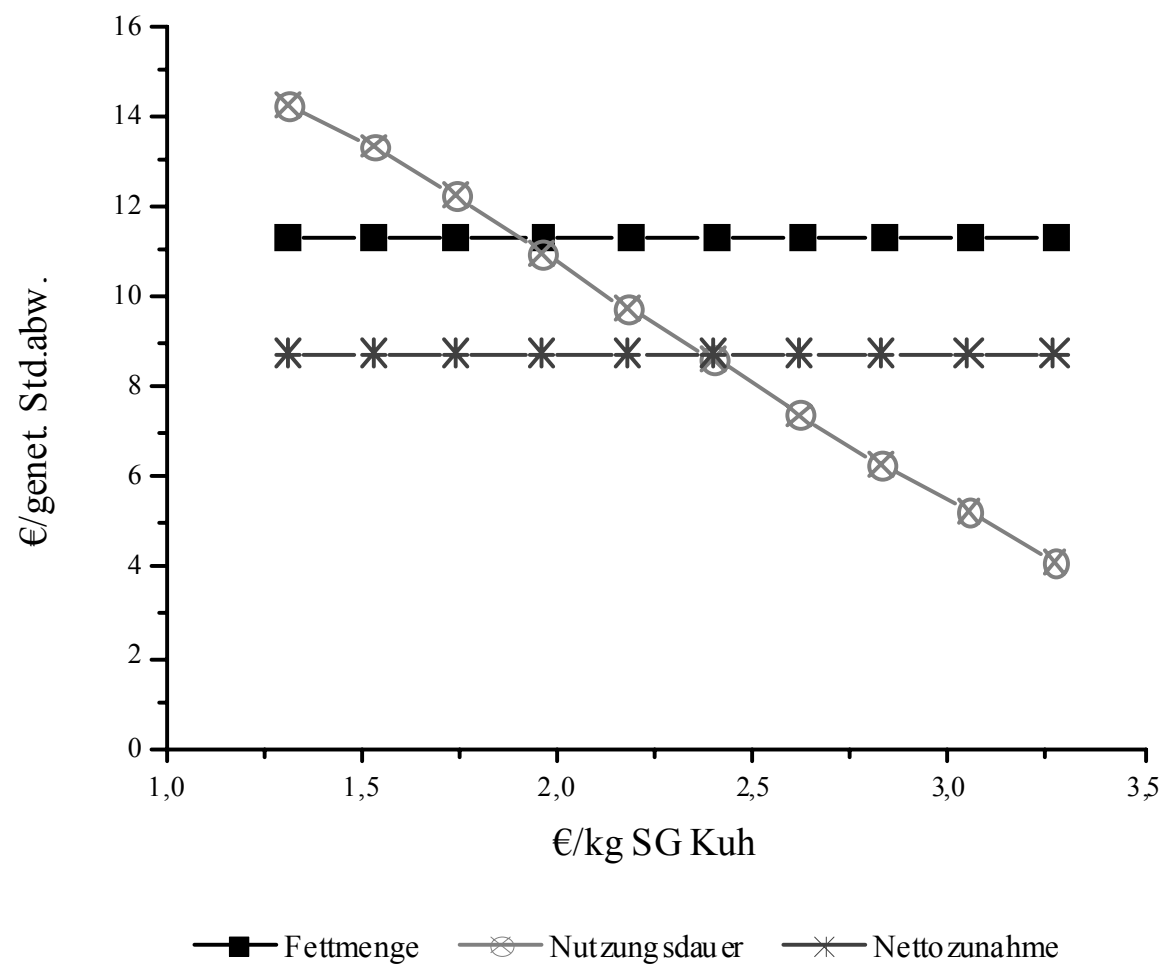

Abb. 4.16 Einfluss des Schlachtpreises für Kühe ( $€$ je $\mathrm{kg} S G$ ) auf die wirtschaftlichen Gewichte der Merkmale Fettmenge, Nutzungsdauer (ND) und Nettozunahme (NTZ) in $€$ je genetischer Standardabweichung im Referenzszenario bei Fleckvieh

Stellvertretend für jeden Merkmalskomplex sind die Auswirkungen veränderter Schlachtpreise für Kühe auf die wirtschaftlichen Gewichte der Merkmale Fettmenge, Nutzungsdauer und Nettozunahme aufgeführt (Abb. 4.16). Ein verbesserter Schlachtpreis nimmt weder auf das wirtschaftliche Gewicht des Merkmals Fettmenge noch auf das des Merkmals Nettozunahme Einfluss. Allerdings wird das wirtschaftliche Gewicht der Nutzungsdauer mit verbessertem Schlachtpreis der Kühe vermindert. Erhält man für eine Schlachtkuh einen höheren Verkaufserlös, so wird die Verbesserung der Nutzungsdauer wirtschaftlich unbedeutender. Der positive Nutzen ei- 
ner verbesserten Nutzungsdauer kann den erhöhten Gewinn aus dem Schlachtviehverkauf nicht aufwiegen und so geht das wirtschaftliche Gewicht mit steigender Nutzungsdauer zurück.

Da die Totgeburtenrate ein hohes wirtschaftliches Gewicht im Vergleich zu den anderen Fitnessmerkmalen aufweist, soll am Beispiel des Schlachtpreises für Mastbullen der Einfluss auf die Totgeburtenrate überprüft werden (Abb. 4.17).

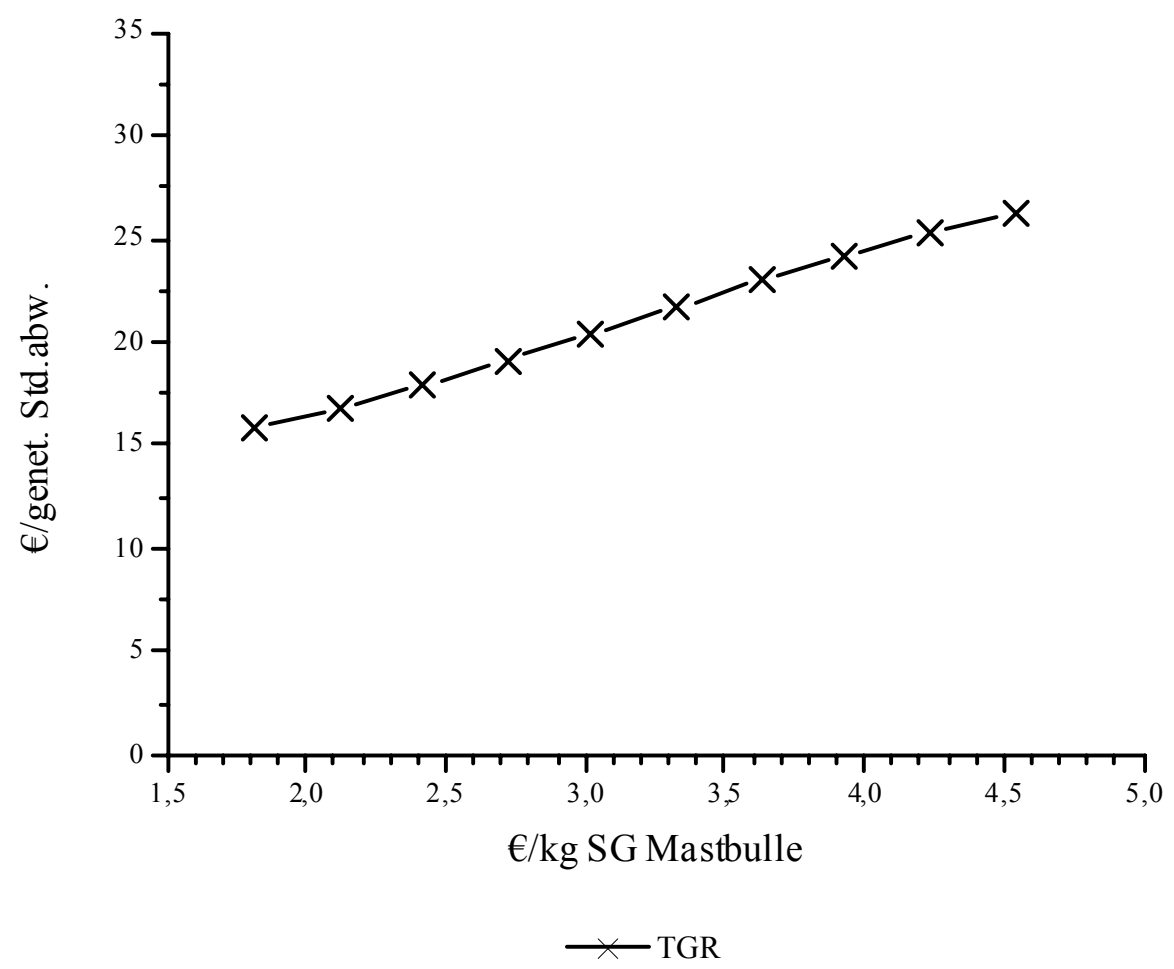

Abb. 4.17 Einfluss des Schlachtpreises für Mastbullen ( $€$ je $\mathrm{kg} S G$ ) auf das wirtschaftiche Gewicht der Totgeburtenrate in $€$ je genetischer Standardabweichung im Referenzszenario bei Fleckvieh

Wie in Abb. 4.17 aufgeführt, nimmt das wirtschaftliche Gewicht für die Totgeburtenrate mit steigendem Schlachtpreis für Mastbullen zu. Gleiches ist bei diesem Merkmal bei Veränderung der Verkaufspreise für Kälber sowie der Schlachtpreise für Kühe und Färsen im Referenzszenario zu beobachten. Darin begründet ist auch das hohe wirtschaftliche Gewicht in den einzelnen Szenarien, da hohe Schlachtpreise sowohl für Kühe als auch für Bullen und hohe Verkaufspreise für Kälber unterstellt sind. So 
ist die Beurteilung der wirtschaftlichen Gewichte der Totgeburtenrate unter der jeweiligen Zukunftseinschätzung kritisch zu betrachten.

\subsubsection{Braunvieh}

Das Braunvieh ist die dritte Rasse, für die im deutschen und österreichischen Zuchtgebiet wirtschaftliche Gewichte abgeleitet werden. Die Berechnungen sind analog zu Holstein und Fleckvieh durchgeführt. Ebenso wie bei Fleckvieh wird im Referenzszenario ein gespreizter Milchpreis angenommen, im Vergleichsszenario ohne Quote liegt der Erzeugerorientierungspreis in Bayern aus 2006 zugrunde.

Da die Einflüsse verschiedener Parameter auf die wirtschaftlichen Gewichte bereits am Bespiel der Rassen Holstein und Fleckvieh dargestellt wurden und Braunvieh die kleinste der drei untersuchten Population in Deutschland und Österreich ist, werden im Folgenden nur die berechneten Grenznutzen und wirtschaftlichen Gewichte in den zwei Szenarien vorgestellt.

Um auch hier die einzelnen Merkmale miteinander vergleichen zu können, sind in Tab. 4.4 die genetischen Standardabweichungen der einzelnen Merkmale bei Braunvieh dargestellt. 
Tab. 4.4 Genetische Standardabweichung der einzelnen Merkmale bei Braunvieh (Dodenhoff, 2006)

\begin{tabular}{lcc}
\hline & Merkmalseinheit & Genetische Standardabweichung \\
\hline Milchträger & $\mathrm{kg}$ & 543 \\
Fettmenge & $\mathrm{kg}$ & 21,2 \\
Eiweißmenge & $\mathrm{kg}$ & 17,0 \\
Nutzungsdauer & Tage & 180 \\
Fruchtbarkeit & $\%$ & 4,5 \\
Totgeburtenrate & $\%$ & 4,0 \\
Kalbeverlauf & Klasse & 0,22 \\
Persistenz & $\sigma$ & 2,5 \\
SCS & Klasse & 0,5 \\
Melkbarkeit & kg/Min & 0,29 \\
Exterieur & Punkte & 2,28 \\
Nettozunahme & $\mathrm{g}$ & 26,5 \\
Handelsklasse & Klasse & 0,25 \\
Ausschlachtung & $\%$ & 1,15 \\
\hline
\end{tabular}

Zunächst sind in Abb. 4.18 die Grenznutzen in $€$ je Merkmalseinheit sowie deren Standardabweichungen bei Braunvieh im Referenzszenario aufgeführt. 
Referenzszenario

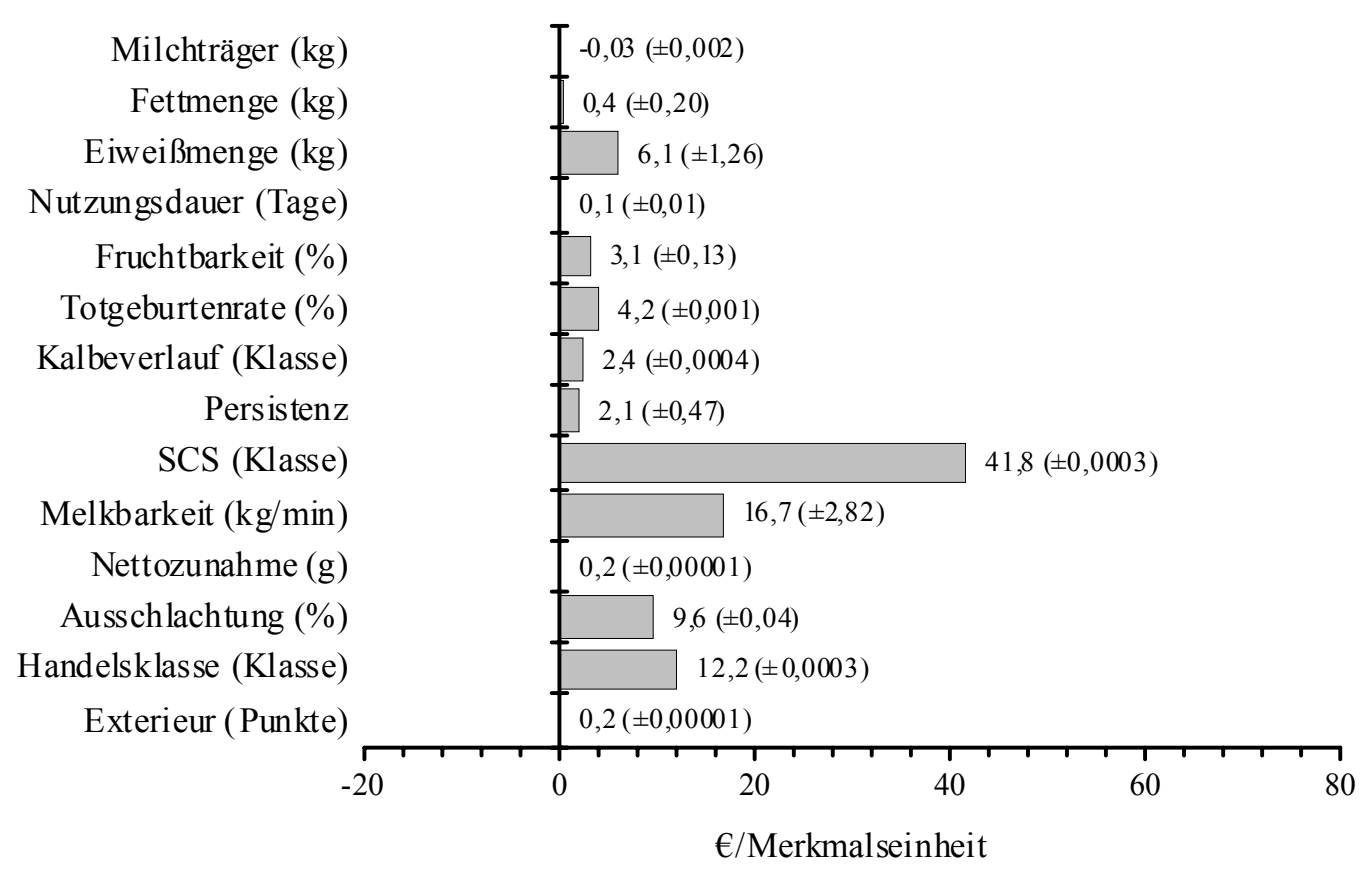

Abb. 4.18 Grenznutzen ( \pm Standardabweichung) der einzelnen Merkmale in $€$ je Merkmalseinheit im Referenzszenario bei Braunvieh

Es zeigt sich, dass der Grenznutzen für den Milchträger mit - $0,03 €$ je kg im negativen Bereich liegt (Abb. 4.18). Die Fettmenge hat einen Grenznutzen von 0,4€ je kg und die Eiweißmenge von $6 €$ je kg. Dementsprechend beträgt die Gewichtung von Fett- zu Eiweißmenge 1:15,25. Dies beruht auf der starken Spreizung des Milchpreises, bei dem die Eiweißmenge 6,7fach höher bezahlt wird als die Fettmenge.

Die Nutzungsdauer erzielt einen Grenznutzen von $0,1 €$ je Tag. Die übrigen Fitnessmerkmale Fruchtbarkeit, Totgeburtenrate, Kalbeverlauf und Persistenz bewegen sich zwischen $2 €$ und $4 €$ je Merkmalseinheit. Verbessert man den SCS um eine Klasse, so erhält man einen Zusatznutzen von etwa $42 €$ je Klasse, bei der Melkbarkeit erzielt man einen Zusatznutzen von etwa $17 €$ je zusätzlichen kg pro Minute. Zusätzlich wird der Grenznutzen für die Fleischleistungsmerkmale abgeleitet. Die Nettozunahme hat einen Grenznutzen von 0,2€ je g, die Ausschlachtung von 9,6€ je \% und die Handelsklasse von 12,2€ je Klasse. Als letztes Merkmal wurden nach dem neu gewählten Ansatz der Grenznutzen für das Merkmal Exterieur berechnet. Dieses hat einen Wert von $0,2 €$ je verbessertem Exterieurpunkt. 
Um die Grenznutzen der einzelnen Merkmale miteinander vergleichen zu können, werden im Folgenden die wirtschaftlichen Gewichte je genetischer Standardabweichung ausgewiesen (Abb. 4.19).

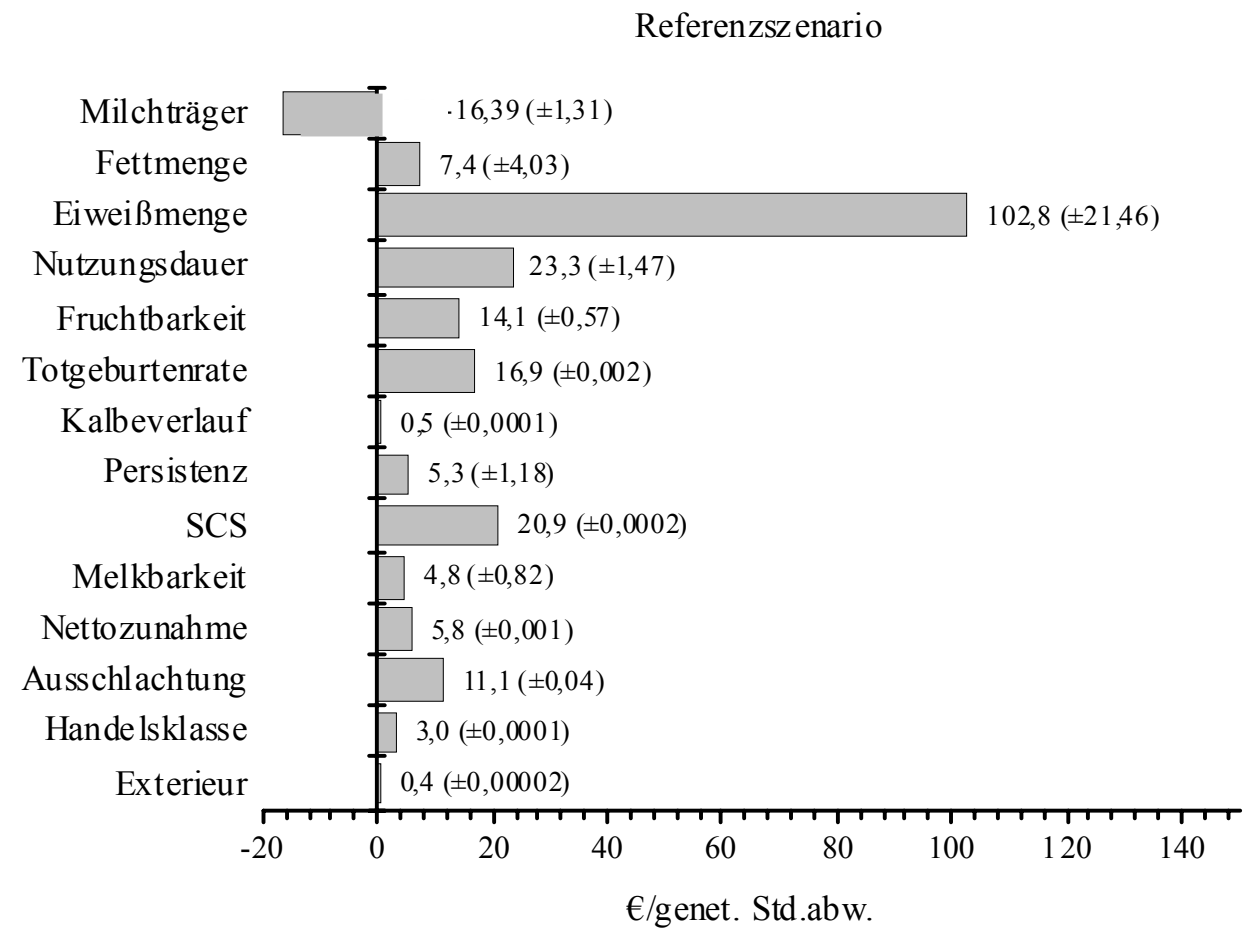

Abb. 4.19 Wirtschaftliche Gewichte ( \pm Standardabweichung) der einzelnen Merkmale in $€$ je genetischer Standardabweichung im Referenzszenario bei Braunvieh

Beim Vergleich der Merkmale untereinander zeigt sich, dass die Eiweißmenge mit Abstand das höchste wirtschaftliche Gewicht und damit auch die größte wirtschaftliche Bedeutung im Referenzszenario hat (Abb. 4.19). Der Milchträger hat ein negatives wirtschaftliches Gewicht von $-16 € / \sigma_{a}$ und die Fettmenge ist mit $7 € / \sigma_{a}$ positiv gewichtet. Bei den Fitnessmerkmalen haben die Merkmale Nutzungsdauer und SCS mit $23 € / \sigma_{\mathrm{a}}$ und $21 € / \sigma_{\mathrm{a}}$ das höchste wirtschaftliche Gewicht, gefolgt von der Totgeburtenrate $\left(17 € / \sigma_{a}\right)$, Fruchtbarkeit $\left(14 € / \sigma_{a}\right)$, Persistenz, Melkbarkeit und Kalbeverlauf. Ähnlich wie bei Fleckvieh ist auch hier das wirtschaftliche Gewicht für die Totgeburtenrate vergleichsweise hoch. Auch bei dieser Rasse zeigt sich der hohe Einfluss der Schlacht-, Verkaufs- und Kälberpreise auf die Wirtschaftlichkeit dieses Merkmals. Die Fleischleistungsmerkmale sind mit einem wirtschaftlichen Gewicht 
zwischen $3 € / \sigma_{\mathrm{a}}$ und $11 € / \sigma_{\mathrm{a}}$ im Vergleich gering. Ebenso verhält sich das wirtschaftliche Gewicht für Exterieur mit $0,4 € / \sigma_{a}$.

In Abb. 4.20 sind die Grenznutzen für Braunvieh im Vergleichsszenario mit Erzeugerorientierungspreis in Bayern ohne Quote dargestellt.

\section{Vergleichsszenario ohne Quote}

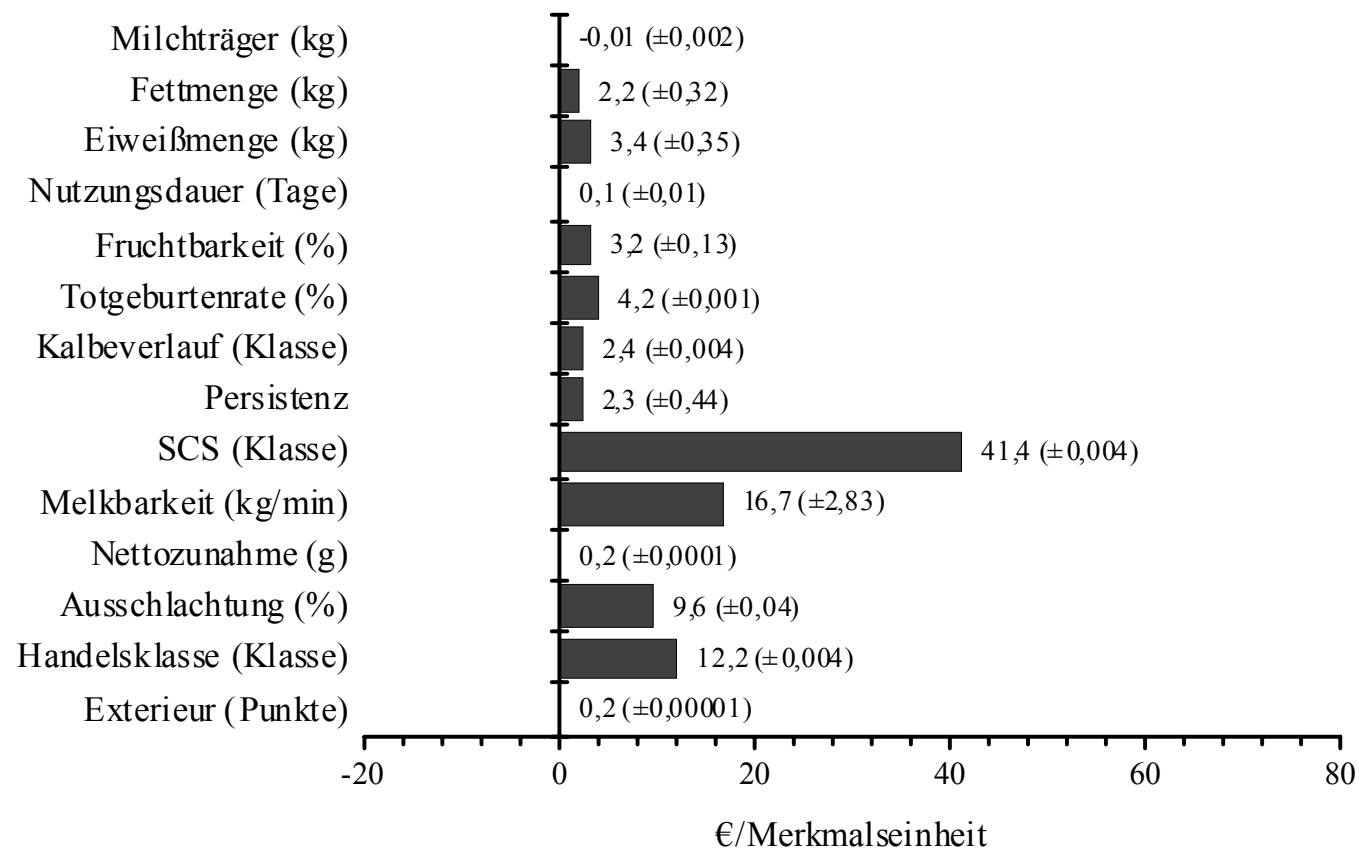

Abb. 4.20 Grenznutzen ( \pm Standardabweichung) der einzelnen Merkmale in $€$ je Merkmalseinheit im Vergleichsszenario bei Braunvieh

Zunächst fällt auf, dass sich das Verhältnis von Fett- zu Eiweißmenge auf 1:1,55 reduziert (Abb. 4.20). Der Milchauszahlungspreis selbst hat ein Bezahlungsverhältnis von 1:1,52. Der Grenznutzen des Milchträgers bleibt allerdings weiterhin negativ. Die funktionalen Merkmale sind kaum von dem veränderten Milchauszahlungspreis beeinflusst, ebenso wie die Fleischleistungsmerkmale.

Die wirtschaftlichen Gewichte in $€$ je genetischer Standardabweichung für Braunvieh im Vergleichsszenario ohne Quote sind in Abb. 4.21 dargestellt. 


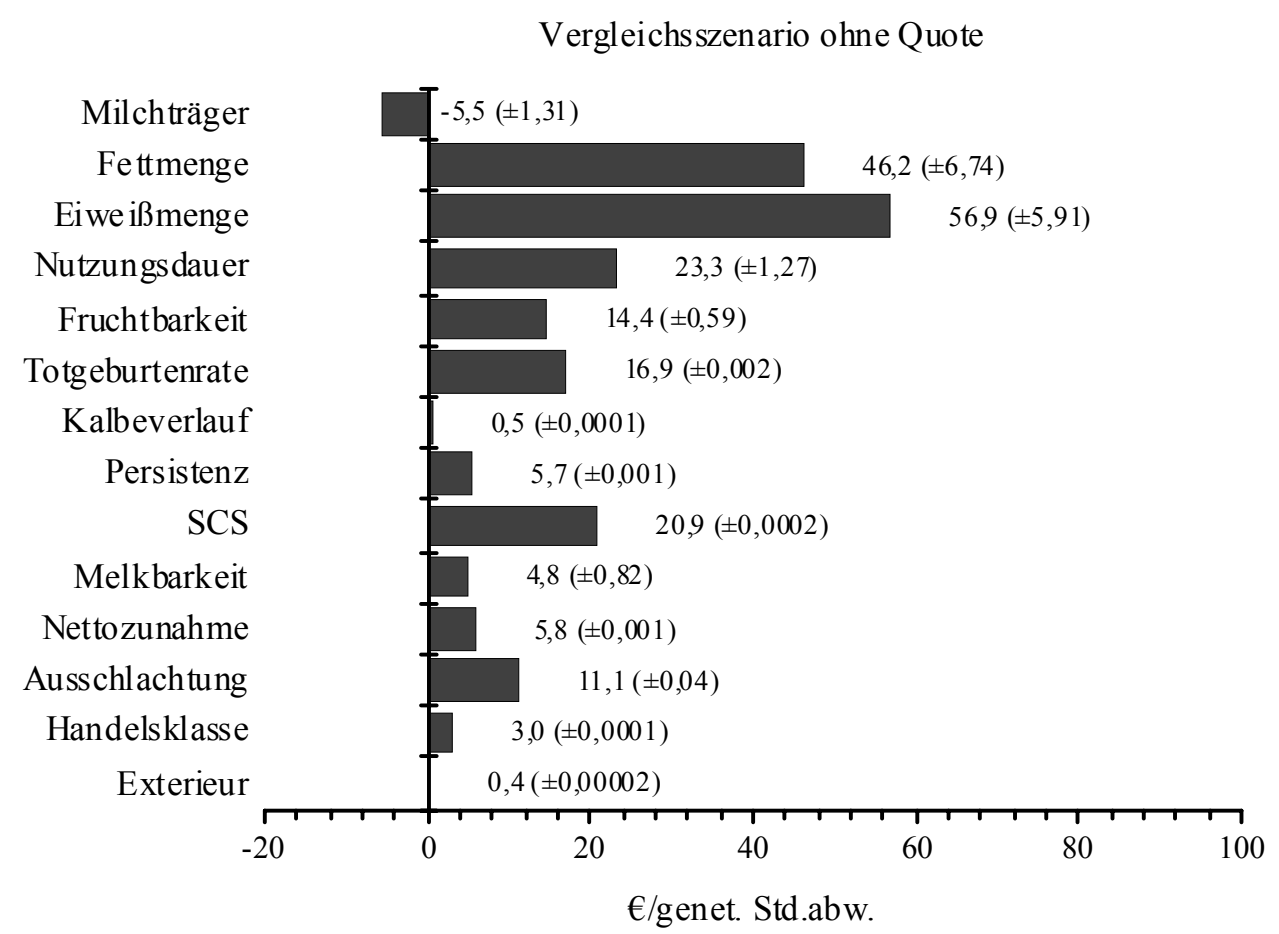

Abb. 4.21 Wirtschaftliche Gewichte ( \pm Standardabweichung) in $€$ je genetischer Standardabweichung im Vergleichsszenario bei Braunvieh

Die wirtschaftlichen Gewichte der Milchleistungsmerkmale sind stark durch den neuen Milchauszahlungspreis beeinflusst (Abb. 4.21). Das wirtschaftliche Gewicht des Milchträgers wird geringer, bleibt aber noch immer negativ. Das wirtschaftliche Gewicht der Fettmenge erhöht sich drastisch auf $46 € / \sigma_{a}$ und das Gewicht der Eiweißmenge reduziert sich auf $60 € / \sigma_{\mathrm{a}}$. Allerdings zeigt der veränderte Milchpreis keinen Einfluss auf die wirtschaftlichen Gewichte der übrigen Merkmale. Diese sind von der Änderung nicht betroffen.

In Abb. 4.22 sind die einzelnen Merkmalskomplexe bei Braunvieh zusammengefasst und für die einzelnen Szenarien dargestellt. 


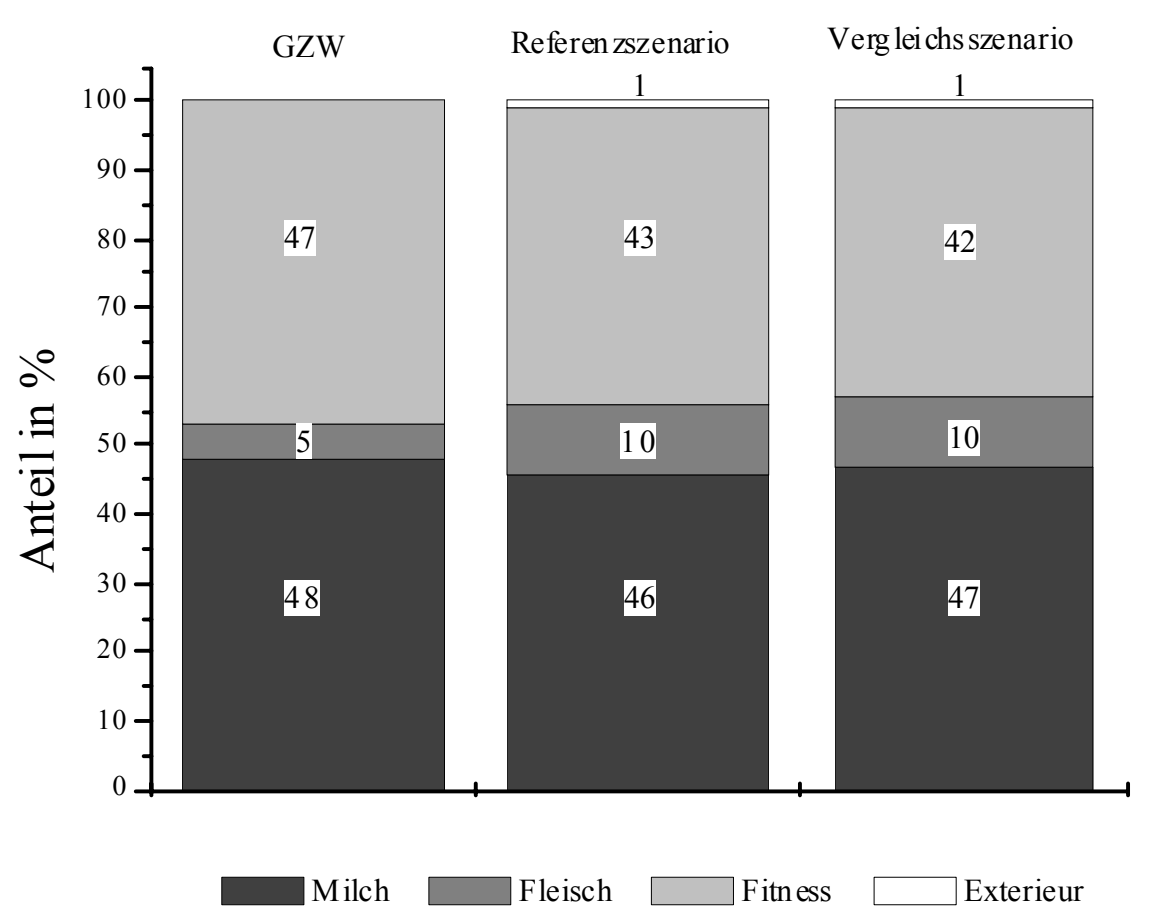

Abb. 4.22 Verhältnis der Merkmalskomplexe bei Braunvieh zueinander (\%) im Referenz- und im Vergleichsszenario ohne Quote

Wie zu sehen ist, hat die Veränderung des Milchpreises bei Braunvieh keinen entscheidenden Einfluss auf die Zusammensetzung und die Konstellation des Gesamtzuchtwertes und der Merkmalskomplexe (Abb. 4.22). Die einzige Veränderung ergibt sich in der Teilzusammensetzung des Komplexes Milch, da sich das Verhältnis von Fett- zu Eiweißmenge verändert. Die Fleischleistungsmerkmale nehmen in beiden Szenarien einen Anteil von 10\% ein.

\subsubsection{Vergleich der drei Rassen}

Abschließend ist in Abb. 4.23 ein Vergleich der wirtschaftlichen Gewichte bei den Rassen Holstein, Fleckvieh und Braunvieh dargestellt. Es werden ähnliche Szenarien für die einzelnen Rassen unterstellt, es wird jeweils ein aktueller Milchauszahlungspreis unterstellt und es gibt keine Milchquotenbeschränkungen. Bei Holstein werden keine wirtschaftlichen Gewichte für die Fleischleistungsmerkmale berechnet, bei Fleckvieh nur für die Fleischleistungsmerkmale Nettozunahme und Handelsklasse 
und bei Braunvieh für alle aufgeführten Merkmale (Abb. 4.23). Ein wirtschaftliches Gewicht für Exterieur wird bei Fleckvieh nicht abgeleitet.

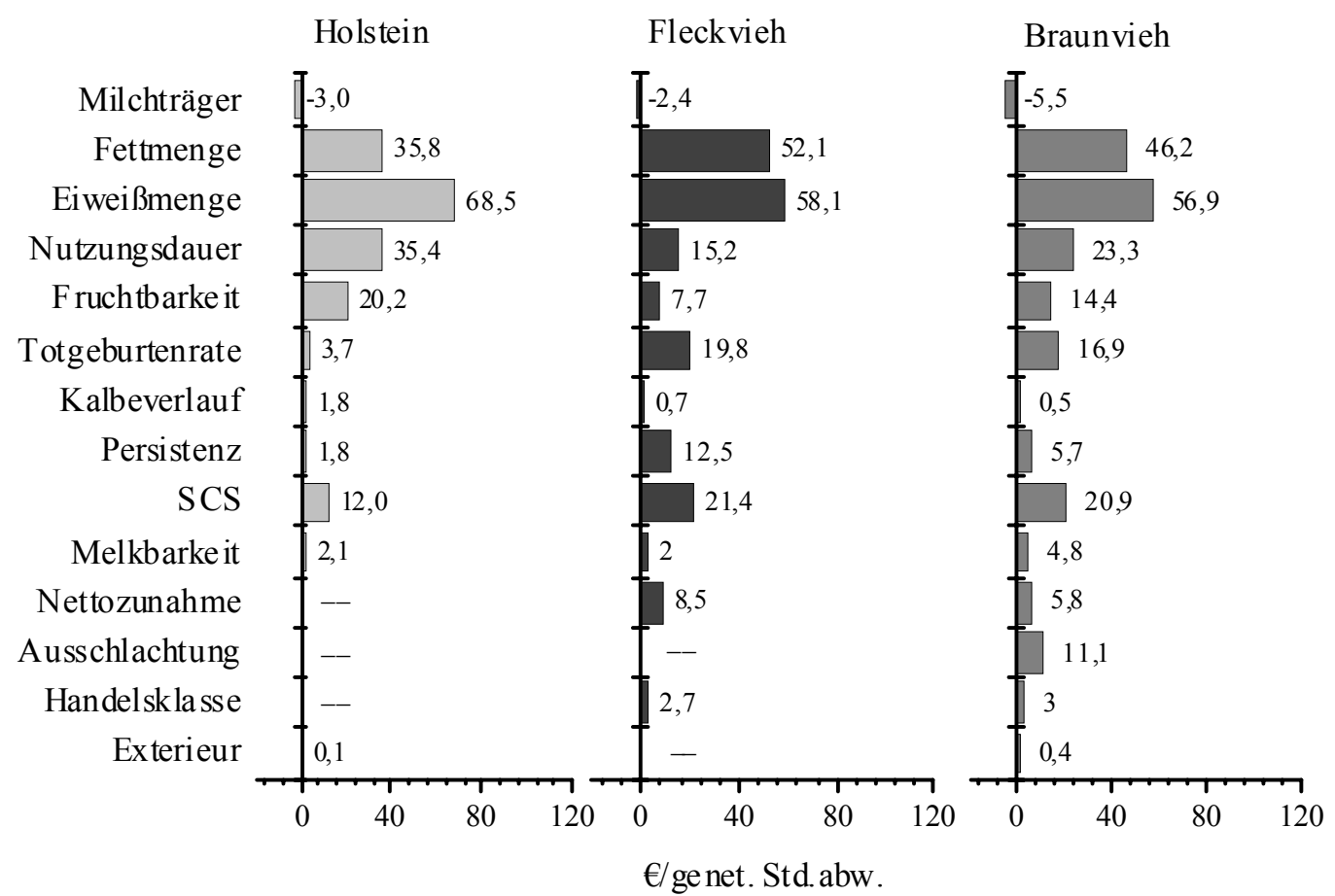

Abb. 4.23 Vergleich der wirtschaftlichen Gewichte in $€$ je genetischer Standardabweichung der Rassen Holstein, Fleckvieh und Braunvieh in Szenarien mit aktuellem Milchauszahlungspreis und ohne Milchquotenbeschränkungen

In allen drei Rassen ist das wirtschaftliche Gewicht des Milchträgers negativ (Abb. 4.23). Die wirtschaftlichen Gewichte für die Fett- und Eiweißmenge sind bei Fleckvieh und Braunvieh ähnlich, bei Holstein ist das Gewicht für die Fettmenge etwas geringer, dafür ist das der Eiweißmenge höher. Dies lässt sich durch die Milchauszahlungspreise erklären, da im norddeutschen Raum die Eiweißmenge höher bezahlt wird als im Erzeugerorientierungspreis in Bayern und die Fettbezahlung leicht geringer ist. Der zusätzliche Nutzen, der bei Erhöhung der Nutzungsdauer entsteht, ist bei der Rasse Holstein am höchsten, bei Fleckvieh am niedrigsten. Beachtet man den Einfluss der durchschnittlichen Nutzungsdauer in der Herde auf die wirtschaftlichen Gewichte, so wird deutlich, dass bei Holstein, mit einer geringeren Nutzungsdauer in der Population, das wirtschaftliche Gewicht am höchsten sein muss. Auch das wirtschaftliche Gewicht für die Fruchtbarkeit ist bei Holstein am höchsten und bei 
Fleckvieh am geringsten. Die Totgeburtenrate ist nicht vergleichbar, da bei Betrachtung der Einzelergebnisse bereits auffällt, dass das wirtschaftliche Gewicht der Totgeburtenrate abhängig von den unterstellten Preisen für Schlacht- und Verkaufstiere ist. Ähnlich verhält sich die Vergleichbarkeit des Merkmals Persistenz. Beim SCS ist das wirtschaftliche Gewicht für die Rasse Holstein am geringsten. Der Zusatznutzen der übrigen Merkmale unterscheidet sich in den einzelnen Rassen nicht deutlich voneinander. Allerdings ist, trotz einer Nichtberücksichtigung im Gesamtzuchtwert, das wirtschaftliche Gewicht des Exterieurs bei Braunvieh höher als bei Holstein, jedoch erreicht es nicht die Bedeutung, die es im Gesamtzuchtwert bei Holstein hat.

\subsection{Indexgewichte am Beispiel Holstein}

Die Ergebnisse der Indexberechnungen werden ausschließlich bei der Rasse Holstein dargestellt. Anhand der Berechnungen wird der Einfluss der wirtschaftlichen Gewichte sowie der Sicherheiten in der Zuchtwertschätzung und der genetischen Parameter auf die Indexgewichte dargestellt.

Die genetische Varianz-Kovarianz-Matrix $(\boldsymbol{G})$ berechnet sich aus der vorgegebenen genetischen Streuung in den Teilzuchtwerten (Kapitel 2.4). Die derzeitige Zusammensetzung des Gesamtzuchtwertes in der Holsteinzucht ist in Tab. 4.5 dargestellt.

Tab. 4.5 Gewichtung der Teilzuchtwerte im Gesamtzuchtwert nach DHV und eigenen Berechnungen

\begin{tabular}{lcc}
\hline & \multicolumn{2}{c}{ Anteile der Teilzuchtwerte am Gesamtzuchtwert (\%) } \\
\cline { 2 - 3 } & DHV & Eigene Berechnung \\
\hline RZM & 50 & 58 \\
RZE & 15 & 1 \\
RZS & 5 & 7 \\
RZN & 25 & 20 \\
RZZ & 5 & 14 \\
\hline
\end{tabular}

In Tab. 4.6 sind die Sicherheiten der Teilzuchtwerte, die wirtschaftlichen Gewichte und Indexgewichte $\left(b^{*} w\right)$ für die derzeitige Gewichtung nach DHV und die Gewich- 
tung mit neu berechneten wirtschaftlichen Gewichten dargestellt. Die Indexgewichte sind für einen Bullen berechnet, der geprüfter Vererber in der Zuchtwertschätzung von November 2006 bei der Rasse Holstein ist.

Tab. 4.6 Relative Gewichtung, Sicherheiten und Indexgewichte $\left(b^{*} w\right)$ der Teilzuchtwerte eines Bullen aus der Top-Liste nach der derzeitigen Zuchtwertschätzung (DHV)

\begin{tabular}{lcccc}
\hline & $\begin{array}{c}\text { Gewichtung der } \\
\text { wirtschaftlichen } \\
\text { Gewichte (\%) }\end{array}$ & $\begin{array}{c}\text { Sicherheiten der } \\
\text { Zuchtwerte }\end{array}$ & Teilzuchtwerte & $b^{*} w$ \\
\hline RZM & 50 & 0,98 & 137,13 & 0,89904 \\
RZE & 15 & 0,85 & 145,54 & 0,02708 \\
RZS & 5 & 0,80 & 94,69 & 0,13141 \\
RZN & 25 & 0,80 & 120,18 & 0,31827 \\
RZZ & 5 & 0,80 & 108,18 & 0,22870 \\
\hline RZG & & & 147,28 & \\
\hline
\end{tabular}

Zum Vergleich sind in Tab. 4.7 die Gewichtung der Teilzuchtwerte, die Sicherheiten und die Indexgewichte $\left(b^{*} w\right)$ für den gleichen Bullen mit geänderter Gewichtung der Teilzuchtwerte zueinander dargestellt. 
Tab. 4.7 Relative Gewichtung, Sicherheiten und Indexgewichte $\left(b^{*} w\right)$ der Teilzuchtwerte eines Bullen aus der Top-Liste nach den eigenen Berechnungen

\begin{tabular}{lcccc}
\hline & $\begin{array}{c}\text { Gewichtung der } \\
\text { wirtschaftlichen } \\
\text { Gewichte (\%) }\end{array}$ & $\begin{array}{c}\text { Sicherheiten der } \\
\text { Zuchtwerte }\end{array}$ & Teilzuchtwerte & $b^{*} w$ \\
\hline RZM & 58 & 0,98 & 137,13 & 0,78004 \\
RZE & 1 & 0,85 & 145,54 & 0,24415 \\
RZS & 7 & 0,80 & 94,69 & 0,11398 \\
RZN & 20 & 0,80 & 120,18 & 0,38718 \\
RZZ & 14 & 0,80 & 108,18 & 0,09068 \\
\hline RZG & & & 141,73 & \\
\hline
\end{tabular}

Vergleicht man beide Varianten miteinander, so fällt auf, dass sich die Indexgewichte besonders im Bereich der Komplexe RZM, RZE und RZZ verändern. Dementsprechend fällt der Gesamtzuchtwert RZG um sechs Punkte. Dies liegt an dem hohen Teilzuchtwert RZE, der mit 145 Punkten über drei Standardabweichungen vom Durchschnitt entfernt liegt. Da das relative wirtschaftliche Gewicht des Exterieurkomplexes in der neuen Berechnung nur 1\% ausmacht, fließt dieser Teilzuchtwert zu einem geringeren Anteil in den RZG ein als zuvor, was in einem niedrigeren Gesamtzuchtwert resultiert.

Eine weitere Variante, den Einfluss auf die Indexgewichte zu überprüfen, ist der Vergleich eines Bullen nach dem Testeinsatz mit einem geprüften Vererber. Die Ergebnisse sind in Tab. 4.8 dargestellt. 
Tab. 4.8 Indexgewichte $\left(b^{*} w\right)$ und Sicherheiten der Teilzuchtwerte im Vergleich bei einem Testbullen nach Testeinsatz und einem geprüften Vererber

\begin{tabular}{lcccc}
\hline & \multicolumn{2}{c}{ Sicherheiten } & \multicolumn{2}{c}{$\mathrm{b}^{*} \mathrm{w}$} \\
\cline { 2 - 5 } & $\begin{array}{c}\text { Testbulle nach } \\
\text { Testeinsatz }\end{array}$ & $\begin{array}{c}\text { geprüfter Verer- } \\
\text { ber }\end{array}$ & $\begin{array}{c}\text { Testbulle nach } \\
\text { Testeinsatz }\end{array}$ & $\begin{array}{c}\text { geprüfter Verer- } \\
\text { ber }\end{array}$ \\
\hline RZM & 0,90 & 0,98 & 0,89510 & 0,89904 \\
RZE & 0,70 & 0,85 & 0,04436 & 0,00271 \\
RZS & 0,75 & 0,80 & 0,14270 & 0,13141 \\
RZN & 0,65 & 0,80 & 0,32389 & 0,31827 \\
RZZ & 0,60 & 0,80 & 0,23424 & 0,22870 \\
\hline
\end{tabular}

Wie in Tab. $4.8 \mathrm{zu}$ sehen, ändern sich die Indexgewichte $\left(b^{*} w\right)$ aufgrund unterschiedlicher Sicherheiten der Teilzuchtwerte. Trotz höherer Differenzen der Sicherheiten im RZN und RZZ, ist der Einfluss der Sicherheiten auf die Indexgewichte beim RZE am höchsten (Tab. 4.8).

In Tab. 4.9 ist das Verhältnis der b-Werte in den einzelnen Teilzuchtwerten der Top10-Bullen zueinander aufgeführt.

Tab. 4.9 Verhältnis der b-Werte in den einzelnen Teilzuchtwerten der Top-10Bullen zueinander (\%)

\begin{tabular}{lccccc}
\hline & $\mathrm{bRZM}^{*} \mathrm{w}$ & $\mathrm{bRZE}{ }^{*} \mathrm{w}$ & $\mathrm{bRZS}^{*} \mathrm{w}$ & $\mathrm{bRZN}^{*} \mathrm{w}$ & $\mathrm{bRZZ}^{*} \mathrm{w}$ \\
\hline DHV & 50 & 19 & 4 & 26 & 1 \\
$9 \%$ RZE & 51 & 12 & 5 & 19 & 13 \\
$1 \%$ RZE & 54 & 4 & 6 & 21 & 15 \\
\hline
\end{tabular}

Es zeigt sich anhand der b-Werte, dass die Gewichtung dieser zueinander von der endgültigen Gewichtung im RZG abweicht. Die b-Werte des RZE sind höher als die eigentliche Gewichtung im Gesamtzuchtwert nach DHV. Auch die b-Werte des RZN sind höher. Dagegen sind die b-Werte der niedrig heritablen Merkmalskomplexe Somatische Zellzahlen und Zuchtleistung geringer. 
Aufgrund der Darstellungen wird deutlich, dass nicht ausschließlich die wirtschaftlichen Gewichte die abschließende Gewichtung des Gesamtzuchtwertes beeinflussen. Vielmehr müssen sowohl die genetischen Parameter als auch die Sicherheiten der Zuchtwertschätzung in den Teilzuchtwerten berücksichtigt werden und durch die Kombination dieser Einzelfaktoren ergibt sich die rechnerische Zusammenstellung des Gesamtzuchtwertes (RZG). Vielfach wird ein direkter Rückschluss von der relativen Gewichtung der wirtschaftlichen Gewichte auf die Zusammensetzung des Gesamtzuchtwertes vorgenommen, was nur zulässig wäre, wenn alle Teilzuchtwerte mit einer Sicherheit von $100 \%$ geschätzt würden. Dies kann nur als Approximation an tatsächliche Berechnungen des Gesamtzuchtwertes dienen (Wünsch, 1999). 


\section{$5 \quad$ Diskussion}

In diesem Kapitel werden vorrangig die Ergebnisse aus den Untersuchungen im Vergleich mit Ergebnissen aus der Literatur diskutiert. Eine Diskussion der in der Ausgangssituation unterstellten Parameter erfolgt in diesem Rahmen nicht mehr. Allerdings ist davon auszugehen, dass eine ausführliche Analyse bereits im Vorfeld stattgefunden hat und die für die Auswertung am sinnvollsten erachteten Parameter berücksichtigt worden sind. Die Grundlagen sind sowohl in der Wissenschaft als auch in der Praxis diskutiert worden.

\subsection{Wirtschaftliche Gewichte}

Um die Ergebnisse zur Ableitung wirtschaftlicher Gewichte angemessen beurteilen zu können, werden im Folgenden sowohl die Methode und das Modell als auch die Ergebnisse mit anderen Arbeiten verglichen und diskutiert. Es bleibt jedoch zu berücksichtigen, dass ein Vergleich von Arbeiten aus unterschiedlichen Untersuchungen schwierig ist, da oft verschiedene Modelle und Methoden angewandt werden und unterschiedliche Annahmen getroffen werden (Reinsch, 1993). Es ist wichtig, die wirtschaftlichen Gewichte abzuleiten, um die Richtung und den Einfluss des jeweiligen Merkmals aufzuzeigen und sie werden des Weiteren benötigt, um optimale Indexgewichte zu erhalten (Veerkamp, 1998).

\subsubsection{Beurteilung des Modells}

Dekkers (1991) stellt fest, dass in Produktionssystemen oft komplexe Zusammenhänge zwischen züchterischen Merkmalen und Produktionsfaktoren vorliegen, die nicht durch eine einfache Gewinngleichung beschrieben werden können. Aus diesem Grund ist es nötig, mit bioökonomischen Modellen zu arbeiten. Es ist wichtig, die wirtschaftlichen Gewichte aus einem optimierten Produktionssystem heraus abzuleiten (Melton et al., 1979). Da im Modell sowohl wirtschaftliche Gewichte für Milchund Fleischleistungsmerkmale sowie funktionale Merkmale abgeleitet werden und somit für alle Merkmale eine Verbesserung im Zuchtziel angestrebt wird, muss dies bei der Auswahl der Methode berücksichtigt werden. Miesenberger (1997) entwickelte das Programm von Amer et al. (1994) weiter, so dass die Bereiche Jungviehaufzucht, Bullenmast und Milchviehhaltung in einem Modell berücksichtigt werden 
können. Für die Ableitung der wirtschaftlichen Gewichte ist es unerheblich, wie groß die Herde auf dem Betrieb ist. Die Herdengröße beeinflusst lediglich Veränderungen im Betriebsgewinn, hat aber keinerlei Einfluss auf die wirtschaftlichen Gewichte. Die stellen bereits Albera et al. (2004) in ihrer Untersuchung über das Zuchtziel bei der Rasse Piemonteser fest, ebenso wie Groen (1989b).

Wie bei Amer et al. (1994), vorgeschlagen wird auch im in dieser Arbeit verwendeten Modell davon ausgegangen, dass die Erlöse und Kosten unabhängig von der genetischen Veränderung sind. Allerdings kann dies in einigen Fällen seine Gültigkeit verlieren. Des Weiteren muss berücksichtigt werden, dass bei der Berechnung der wirtschaftlichen Gewichte komplexe Zusammenhänge im biologischen Bereich nicht einbezogen werden. Eine Schwergeburt hat z. B. durchaus Auswirkungen auf die weitere Fruchtbarkeit der Kuh und damit auch die Wirtschaftlichkeit, allerdings kann dieser komplexe Zusammenhang im diesem Modell nicht implementiert werden. Auch ist auf die Doppelzählung zu achten, da viele Zusammenhänge bereits in die Wirtschaftlichkeit eines anderen Merkmals hinein spielen. Wie bei Nielsen (2004) beschrieben, kommen die hauptsächlichen Erlöse des Betriebes aus dem Verkauf von Milch, dem Verkauf überzähliger Färsen und dem Verkauf von Mastbullen und gemerzten Kühen. Die Kosten setzen sich aus den Aufwendungen für Fütterung, Krankheiten, Kalbungen, Melkarbeit, Besamung und Remontierung sowie Aufwendungen für die Erstellung von Mastbullen zusammen. Auch im vorliegenden Modell ist dies der Fall.

Bei den Gewinngleichungen und Modellen in der Literatur werden überwiegend aktuelle Bedingungen unterstellt (Gibson und Wilton, 1998). Berücksichtigt man die Zeitspanne, die Züchtung benötigt, um umgesetzt zu werden, ist es unerlässlich bei der Konzipierung eines Modells zukünftige Zustände zu beschreiben und zu unterstellen. Das Modell sollte den Zeitpunkt beschreiben, an dem der züchterische Fortschritt eintritt. Mit der Weiterentwicklung der Methode soll in der vorliegenden Arbeit den Unsicherheiten in den zukünftigen Markt- und Preisentwicklungen besser Rechnung getragen werden. Wie an den Ergebnissen deutlich wird, hat die Annahme eines Quotenszenarios oder die Veränderung der Zusammensetzung des Milchauszahlungspreises einen großen Einfluss auf die Höhe und Ausrichtung der wirtschaftlichen Gewichte. Daher ist es wichtig, ein möglichst realitätsnahes Abbild der $\mathrm{Zu}$ kunft zu konzipieren. Gibson und Wilton (1998) stellen fest, dass die Nachfrage nach Agrarprodukten in Zukunft einer Sättigung zustrebt. Damit würde eine Verbesserung der Genetik der Tiere dazu führen, dass im Betrieb mit weniger Tieren die gleiche Milchmenge produziert würde. Amer und Fox (1992) zeigen, dass die Beziehungen zwischen Angebot und Nachfrage, Produktionskosten und Preisen berücksichtigt werden sollten. Da in der Tierzucht das genetische Niveau einer Population je nach 
Generationsintervall nur mittel- bis langfristig verändert werden kann, müssen zukünftige Gleichgewichtspreise bekannt sein. Weil dies aber nicht der Fall ist, werden aktuelle Preise verwendet. Allerdings sollten die Züchter bei der endgültigen Festsetzung des Zuchtziels trotz allem darauf achten, wie die Marktprognosen sind (Habier et al., 2004). Der Verbraucher wird mittel- und langfristig bei der Einschätzung der zukünftigen Marktsituation immer ein Unsicherheitsfaktor bleiben.

Zur Beschreibung der Laktationskurve werden die Wood-Parameter verwendet (Wood, 1967). In der Zuchtwertschätzung wird die Ali-Schaeffer-Funktion (Ali und Schaeffer, 1987) angewendet. Guo (1998) untersucht verschiedene Modelle zur Bestimmung der Laktationskurve. Viele Modelle unterscheiden sich nur in ihrer Anpassung und es bestehen nur geringe Differenzen in der Aussagekraft. Es zeigt sich, dass für die Zuchtwertschätzung das Ali-Schaeffer-Modell (Ali und Schaeffer, 1987) am günstigsten zu sein scheint, für das hier zugrunde liegende Modell aber die Anpassung der Laktationskurve mit Hilfe der Wood-Parameter (Wood, 1967) durchaus angemessen scheint. Auch Nielsen (2004) verwendet zur Beschreibung der Laktationskurve die Wood-Funktion (Wood, 1967).

Im SimHerd-Modell von Nielsen (2004) wird die Berechnung der wirtschaftlichen Gewichte in jedem Szenario 1000 mal wiederholt. Durch diese Vorgehensweise wird versucht, die Managementeinflüsse und die Problematiken des jeweiligen Produktionssystems auszuschalten. Dadurch werden für das jeweilige wirtschaftliche Gewicht ein Mittelwert und eine Standardabweichung berechnet. Im vorliegenden Modell werden der Mittelwert und die Standardabweichung des wirtschaftlichen Gewichtes für ein Merkmal durch die Veränderung eines Populationsparameters hervorgerufen. Auf diese Weise erhält man eine große Anzahl von Daten, die mit der jeweiligen Eintrittswahrscheinlichkeit gewichtet werden können. Hintergrund dieser Vorgehensweise ist die Berücksichtigung unsicherer Faktoren, die auf diese Weise besser quantifiziert werden können und deren Einfluss auf das jeweilige Szenario damit von vornherein eingeordnet werden kann. Des Weiteren weist Nielsen (2004) darauf hin, dass die Standardabweichungen der wirtschaftlichen Gewichte die Variation des realisierten Gewinnes im Betrieb, vor und nach der Verbesserung des Merkmals, darstellen.

Eine Erweiterung des Modells um die Merkmale SCS, Melkbarkeit, Nettozunahme und Exterieur ist notwendig, um wirtschaftliche Gewichte für alle wichtigen Merkmale in der Zuchtwertschätzung ableiten zu können. Voraussetzung hierbei ist eine korrekte Einbindung der Merkmale in das Modell und die Annahme realistischer Ertrags- und Kostenkomponenten. Nachteilig ist, dass die hohe Komplexität, die gerade bei den funktionalen Merkmalen vorherrscht, nicht in ihrer Gänze berücksichtigt werden kann. So sind die negativen gesundheitlichen Folgen einer Erkrankung der Milchkuh nicht durch eine veränderte Fruchtbarkeit zu berücksichtigen und lediglich 
der Einfluss auf die Milchleistung kann herausgestellt werden. Bei diesen komplexen Darstellungen würden sich eher andere Modelle, wie die Lineare oder Dynamische Programmierung, eignen, um dies in ausreichendem Maße berücksichtigen zu können.

\subsubsection{Einschätzung der Szenarien}

Das Referenzszenario geht aus den Daten der Betriebszweigauswertung in den Ländern Schleswig-Holstein und Bayern (Landwirtschaftskammer, 2004; LfL, 2004a) hervor. Es wird darauf geachtet, dass die Annahmen realitätsnah getroffen sind und die zukünftige Situation in der Landwirtschaft widerspiegeln. Problematisch gestaltet sich die Situation bei Braunvieh, da dort eine schlechte Datengrundlage bezüglich der Bullenmast vorliegt. Da die Fleischleistungsmerkmale jedoch in der Zuchtwertschätzung mit berücksichtigt werden, ist auch für diese Merkmale ein wirtschaftliches Gewicht abzuleiten. Anhand der Daten des BAFF (2006) sind die Parameter näherungsweise bestimmt worden und reflektieren die Bullenmast bei Braunvieh. Nielsen (2004) definiert das simulierte Szenario so, dass es die Produktion im Jahr 2012 widerspiegelt. Zu diesem Zeitpunkt drückt sich die genetische Verbesserung aus, deren Züchtung zum aktuellen Zeitpunkt ausgelöst wurde.

Für den Milchauszahlungspreis sind für die verschiedenen Rassen unterschiedliche Ausgangssituationen angenommen worden. In Norddeutschland wurde für Holstein der Milchauszahlungspreis der Nordmilch angenommen. Da die Molkereienlandschaft in Norddeutschland von großen Genossenschaftsmolkereien geprägt ist, wird auch für die Zukunft davon ausgegangen, dass die Struktur weitestgehend erhalten wird bzw. sich weiterhin Molkereien zusammenschließen. Es ist zu beobachten, dass die Genossenschaftsmolkereien in großer Menge Standardware produzieren und dies auch weiterhin verfolgen werden. Daher erscheint es sinnvoll, den Preis auch unter zukünftigen Bedingungen anzunehmen. In Süddeutschland gestaltet sind die Struktur unter den Molkereien anders. Es gibt viele Privatmolkereien, es wird Markenware, bevorzugt im Käsesektor, produziert. Daher erscheint es sinnvoll, den Wandel, den Seufferlein (2005) in Süddeutschland bereits andeutet, weiterzuführen und eine große Spreizung zwischen Fett- und Eiweißauszahlungspreis zu unterstellen. Dies führt zu dem verwendeten Milchauszahlungspreis bei Fleckvieh und Braunvieh, der aber unter den diskutierten Annahmen sinnvoll erscheint. Als Alternative wird im Vergleichsszenario der aktuelle Erzeugerorientierungspreis in Bayern angenommen. Zusätzlich wird nur bei der Ableitung der wirtschaftlichen Gewichte für Fleckvieh das Vergleichsszenario mit Milchquote und Referenzfettgehalt betrachtet. Dies spiegelt die derzeitig in Deutschland gegebene Situation in der Milchproduktion wider. 
Mack et al. (1997) stellen eine Vorausschätzung zukünftiger Produkt- und Faktorpreise an, indem vergangene Entwicklungstendenzen der EU-Agrarreformen berücksichtigt werden und die erwarteten Einflüsse auf die ermittelten Ergebnisse untersucht werden. Für das Milchleistungsniveau werden Leistungssteigerungen von 100 kg Milch pro Kuh und Jahr angenommen. Des Weiteren geben Mack et al. (1997) zu bedenken, dass eine Leistungsverbesserung in der Rinderzucht unwillkürlich die Gesamtproduktion an Milch und Fleisch steigern wird und die davon ausgehenden Preiseffekte tendenziell zu einer Verminderung der einzelbetrieblichen Grenznutzen führen. Wolfová et al. (2006) zeigen in ihren Berechnungen zu Fleischrassen, dass die wirtschaftlichen Gewichte der Produktionsmerkmale nicht von staatlichen Beihilfen beeinflusst werden.

Nielsen (2004) stellt fest, dass die wirtschaftlichen Gewichte zwischen den einzelnen untersuchten Szenarien sehr ähnlich sind. Es wird sowohl ein Szenario unterstellt, das die derzeitige Milchproduktion in Dänemark widerspiegelt, als auch ein Trendszenario, das zukünftig angenommene Preise und Marktsituationen einbezieht. Im Szenario ohne Milchquotenregelungen nimmt die relative Bedeutung der Milchleistungsmerkmale gegenüber den funktionalen Merkmalen zu. Die gleiche Situation kann man in der vorliegenden Untersuchung feststellen. Bei Fleckvieh zeigt sich deutlich, dass im Vergleichsszenario mit Milchquote der relative Anteil der Milchleistungsmerkmale zugunsten der funktionalen Merkmale dramatisch zurückgeht. Die Unterschiede zwischen den Szenarien ergeben sich hauptsächlich aus den unterschiedlich angenommenen Preisen bei Milch und Fleisch. Daher haben die Szenarien auf die Kosten und Erlöse der funktionalen Merkmale und damit auf die wirtschaftlichen Gewichte Einfluss. Groen (1989b) zeigt, dass die Veränderungen im Preisniveau der Produkte ausschließlich die wirtschaftlichen Gewichte der Merkmale betrifft, die den Output der entsprechenden Produkte beeinflusst. Der Gewinn im Betrieb geht zurück, wenn die Preise für Milch und Schlachtkühe niedrig und die Futterkosten hoch sind (Jagannatha et al., 1998). Die Bedeutung der Produktionsmerkmale ist höher, wenn die Futterkosten gering sind, da das Futter den größten Anteil der Kosten im Betrieb hat.

Keller und Allaire (1990) weisen darauf hin, dass unter verschiedenen Modellkalkulationen die Rasse Holstein unter Bedingungen eines mengenmäßig ausgerichteten Milchmarktes deutliche Vorteile hat, während die Rassen Jersey und Guernsey in einem Milchmarkt mit auf Komponenten ausgerichteter Bezahlung überlegen sind. Ist der Markt auf die Käseproduktion ausgerichtet, haben die Rassen Brown Swiss und Ayrshire deutlichen im Vorteil in der Wirtschaftlichkeit des Betriebes.

Anhand der Untersuchungen von Pieters et al. (1997) zeigt sich, dass die relativen wirtschaftlichen Gewichte für das unterstellte Szenario nur gering durch Veränderun- 
gen im Preis- und Produktionsniveau beeinflusst sind. Allerdings weisen sie auch auf die Unterschiede zwischen dem Szenario mit und ohne Quotenbeschränkungen hin und machen deutlich, dass eine Veränderung der Beschränkungen zu einer neuen Berechnung der wirtschaftlichen Gewichtung und einer gezielten Betrachtung des Zuchtziels führen sollten. Dies kann auch in der vorliegenden Arbeit bestätigt werden.

Wünsch (1999) berechnet wirtschaftliche Gewichte für die sächsische Milchrinderpopulation. Neben den Milchleistungsmerkmalen werden auch Gewichte für die funktionalen Merkmale abgeleitet. Das höchste wirtschaftliche Gewicht hat die Eiweißmenge mit über $40 € / \sigma_{a}$. Ebenfalls ein hohes wirtschaftliches Gewicht mit fast $40 € / \sigma_{\mathrm{a}}$ hat die Nutzungsdauer. Man muss jedoch beachten, dass die wirtschaftlichen Gewichte unter Annahme einer Milchquotenregelung abgeleitet sind, wohingegen die wirtschaftlichen Gewichte aus den eigenen Untersuchungen unter der Annahme eines freien Marktzuganges berechnet wurden. Allerdings zeigt sich in der Tendenz, dass durch den Wegfall von Quotenregelungen die Gewichtung der Milchleistungsmerkmale ansteigen würden, ebenso wie sich dies in den eigenen Berechnungen darstellt. Zudem erlangt die Fruchtbarkeit eine größere Bedeutung, während sich der Anteil von SCS und Melkbarkeit verringert. Dementsprechend verstärken sich die Gewichte der Merkmale, die einen direkten Einfluss auf die Wirtschaftlichkeit des Betriebes haben.

Es zeigt sich anhand der berechneten Standardabweichungen, dass sich unter der Annahme unsicherer Milchpreise und variierter Milchleistung nur ein nachhaltiger Einfluss auf die Milchleistungsmerkmale nachzuweisen ist. Die Standardabweichungen der funktionalen Merkmale bzw. der Fleischleistungsmerkmale und des Exterieurs liegen in einem niedrigen Bereich, so dass die wirtschaftlichen Gewichte auch für die Zukunft recht robust zu sein scheinen. Die wirtschaftlichen Gewichte sollten je nach Einschätzung der Höhe des Milchauszahlungspreises und der Milchleistung ausgewählt werden.

\subsubsection{Milchquotenregelungen}

Bei Betrachtung der Ergebnisse bei Fleckvieh fällt auf, dass die wirtschaftlichen Gewichte sich unter einer Situation mit Milchmengenbegrenzung verändern. Besonders die Milchleistungsmerkmale sind betroffen. Pieters et al. (1997) untersuchen die Auswirkungen von unterschiedlichen Preissystemen zwischen den Regionen Italiens auf die wirtschaftlichen Gewichte. Dabei stellen sie fest, dass die Begrenzung der Milchliefermenge einen Einfluss auf die Gewichte hat. Es kommt zu Veränderungen in allen Preissystemen. Wie erwartet liegen die größten Gewinne im Falle eines Sze- 
narios ohne Milchmengenbegrenzung vor, wobei die Veränderungen des Fettpreises den geringsten Effekt auf den Betriebsgewinn ausüben. Ähnlich ist auch die Situation in der eigenen Untersuchung. Bekman und van Arendonk (1993) stellen fest, dass die wirtschaftlichen Gewichte für Milchleistungsmerkmale in Szenario mit Quotenbegrenzung niedriger sind als ohne Quotenbegrenzung. Dies zeigt sich auch in den Vergleichszenarien bei Fleckvieh. Mack et al. (1997) zeigen, dass eine Steigerung der Milch- und Fettmenge unter Milchquotenbedingungen nicht wirtschaftlich ist. Eine Steigerung der Eiweißmenge hingegen schon. Der Grenznutzen für die Eiweißmenge beträgt $2,87 €$ je $\mathrm{kg}$ für Fleckvieh und $2,78 €$ je $\mathrm{kg}$ für Holstein. Dies zeigt sich auch bei der eigenen Ableitung der wirtschaftlichen Gewichte bei Fleckvieh. Nielsen (2004) weist darauf hin, dass unter Milchquotenbedingungen die Anzahl der Kühe im Betrieb bei einer Verbesserung der Milchleistung zurückgeht. Unter den gegebenen Berechnungen zeigt sich das besonders deutlich beim wirtschaftlichen Gewicht der Fettmenge bei Fleckvieh im Vergleichsszenario unter Milchquote mit Referenzfettgehalt. Harris und Freeman (1993) zeigen, dass Produktionsquoten die Richtung der wirtschaftlichen Gewichte für Merkmale unter Quote verändern können. Miesenberger et al. (1997) stellen fest, dass die Quotenbedingungen keinen Einfluss auf die wirtschaftlichen Gewichte der Fleischleistungsmerkmale und funktionalen Merkmale haben, was sich auch in den eigenen Berechnungen zeigt. Pärna et al. (2003) weisen ebenfalls darauf hin, dass unter der Voraussetzung einer Milchquote die wirtschaftlichen Gewichte der Milchleistung und der Nutzungsdauer vermindert sind. Pieters et al. (1997) zeigen, dass das relative wirtschaftliche Gewicht der Fettmenge in allen Preissystemen negativ wird, wenn die Produktionsmenge begrenzt ist. Der höchste Gewinn bei Verbesserung der Milchleistung pro Kuh und Jahr um 1 kg kann erzielt werden, wenn keine Produktionsbeschränkung für die Fettmenge vorliegt. Allerdings hatten auch die Veränderungen des Auszahlungspreises für Fett die geringsten Auswirkungen auf die wirtschaftlichen Gewichte.

Albera et al. (2004) regen an, die wirtschaftlichen Gewichte neu zu bearbeiten, wenn eine Veränderung der Quotenregelung eintritt. Um diese Situation für zukünftige Betrachtungen zu implementieren, wurde in den Referenzszenarien von einer Marktsituation ohne Milchmengen- oder Fett- und Eiweißbegrenzung ausgegangen. Pärna et al. (2003) zeigen, dass unter Milchquotenbedingungen und sinkenden Milchpreisen die funktionalen Merkmale, besonders die Reproduktionsmerkmale, einen groBen Einfluss auf die Wirtschaftlichkeit des Betriebes haben und aus diesem Grund in jedes Zuchtprogramm gehören. Pieters et al. (1997) stellen fest, dass die Art der Limitierung der produzierten Produkte den größten Einfluss auf den Erlös des Zuchtprogramms hat. Wünsch und Bergfeld (2001) zeigen, dass die Einführung einer Quote zum Absinken der Grenznutzen und wirtschaftlichen Gewichte des entsprechenden Merkmals, wie auch in den eigenen Berechnungen, kommt. 


\subsubsection{Milchleistungsmerkmale}

In allen Rassen bei hat das Merkmal Eiweißmenge der eigenen Untersuchung das höchste absolute und relative wirtschaftliche Gewicht und auch den größten relativen Anteil. Bereits Rogers (1994), Miesenberger et al. (1997), Mulder und Jansen (2001), Pärna et al. (2003) sowie Pérez-Cabal und Alenda (2003) zeigen, dass die Eiweißmenge den höchsten wirtschaftlichen Beitrag aller Merkmale hat. Dementsprechend nehmen auch in diesen Untersuchungen die Milchleistungsmerkmale den größten Anteil an den wirtschaftlichen Gewichten ein, was auch auf den Milchauszahlungspreis und dessen Zusammensetzung zurückzuführen ist. Durch den hohen Auszahlungspreis für die Komponente Eiweißmenge ergibt sich auch ein entsprechend hohes wirtschaftliches Gewicht für die Eiweißmenge. Besonders deutlich wird dies im Vergleich des Referenzszenarios mit dem Vergleichsszenario bei Fleckvieh. Harris und Freeman (1993) sowie Mack et al. (1997) zeigen, dass die Steigerung der wirtschaftlichen Gewichte bei der Eiweißmenge größer ist als bei der Fettmenge. Dies wird in der vorliegenden Arbeit bestätigt.

Bekman und Van Arendonk (1993) weisen darauf hin, dass die wirtschaftlichen Gewichte der Milchleistungsmerkmale von den Preisstufen der Fett- und Eiweißbezahlung beeinflusst werden, sowohl unter Milchmengenbegrenzung als auch im freien Markt. Dies bestätigt sich auch in der vorliegenden Arbeit. Dekkers (1991) ermittelt einen Grenznutzen für die Milchleistung von $0,44 € \mathrm{je} \mathrm{kg}$, wobei allerdings nicht die Einzelmerkmale Fett- und Eiweißmenge berücksichtigt werden. Der Grenznutzen ist in diesem Fall stark beeinflusst von dem Ausgangsniveau der Herdenleistung und zeigt einen deutlichen Rückgang bei steigender Leistung. Dieser Einfluss ist auch in den vorliegenden Berechnungen festzustellen. Nielsen (2004) berechnet einen Grenznutzen von $0,13 €$ je kg Milch pro Kuh und Jahr. De Jong und Harbers (2001) ermitteln wirtschaftliche Gewichte von $-0,15 €$ je $\mathrm{kg}$ Milch, 39,5€/ $\sigma_{\mathrm{a}}$ für das Merkmal Fettmenge und $175,7 € / \sigma_{\mathrm{a}}$ für das Merkmal Eiweißmenge. Am Beispiel der Szenarien bei Fleckvieh wird deutlich, dass die Milchmengenbegrenzung einen großen Einfluss auf die mit der Milchmenge verbundenen Merkmale ausübt. Mulder und Jansen (2001) untersuchen die Sensitivität der Opportunitätskosten von Quoten und stellen fest, dass diese den größten Einfluss auf die wirtschaftlichen Gewichte von Fett- und Eiweißmenge haben. Groen (1989a) zeigt, dass der Milchpreis einzig die wirtschaftlichen Gewichte der Milchleistungsmerkmale beeinflusst. Kaltenecker (1999) stellt zudem fest, dass mit zunehmender Milchmenge im Betrieb das wirtschaftliche Gewicht von Fett- und Eiweißmenge sinkt, wobei das Gewicht der Fettmenge stärker betroffen ist.

Bei sinkenden Milchpreisen reagieren die wirtschaftlichen Gewichte für die Milchleistungsmerkmale mit einem stärkeren Rückgang als die wirtschaftlichen Gewichte 
der Zuchtleistungsmerkmale (Mack et al., 1997). Insgesamt ist dies bei sinkenden Milch- und Kraftfutterpreisen zu beobachten. Es bleibt aber festzuhalten, dass auch zukünftig Milchleistungssteigerungen wirtschaftlich bleiben und einen entscheidenden Einfluss auf die gesamte Ökonomie des Betriebes haben. Nielsen (2004) zeigt, dass die wirtschaftlichen Gewichte der Merkmale von den Milchquotenregelungen beeinflusst sind, deren Veränderung in einer Erhöhung der Milchleistung je Kuh resultieren. Groen (1989b) zeigt einen deutlichen Einfluss der Futtermittelkosten auf die Höhe der wirtschaftlichen Gewichte der Milchleistungsmerkmale. Werden nur die Milchleistungsmerkmale betrachtet, so bedingt ein hoher Kraftfutterpreis ein günstiges wirtschaftliches Gewicht der Eiweißmenge im Vergleich zu den übrigen Merkmalen. Kaltenecker (1999) stellt in seinen Untersuchungen fest, dass sich die Zuchtzielsetzung in Zukunft zugunsten der Milchmenge und der Eiweißmenge verschiebt während die Gewichtung der Fettmenge und der Fleischleistungsmerkmale zurückgeht. Eine Verschiebung hin zur Eiweißmenge und weg von der Fleischleistung zeigt sich auch in den eigenen Untersuchungen bei der Rasse Fleckvieh. Kaltenecker (1999) ermittelt des Weiteren eine Relation des Grenznutzens von Fett zu Eiweiß von 1:5 und prognostiziert noch eine weitere Spreizung der Merkmale zueinander. Wünsch und Bergfeld (2001) stellen fest, dass sich durch sinkende Auszahlungspreise der Grenznutzen für die Fettmenge vermindert. In der vorliegenden Arbeit wird dies bestätigt.

Es stellt sich jedoch die Frage, wie sinnvoll es ist, neben der Fett- und Eiweißmenge auch das Merkmal Milchträger in das Zuchtziel bzw. den Gesamtzuchtwert zu integrieren. Im Milchauszahlungspreis der Molkereien wird nach wie vor ein Grundpreis für die abgelieferte Milch bezahlt und nicht ausschließlich nur die Fett- und Eiweißmenge. Zudem zeigt sich bei allen drei Rassen, dass der Milchträger immer ein negatives wirtschaftliches Gewicht hat. Dies wirkt sich bei einer weiteren Selektion auf die Milchmenge durchaus negativ auf den Betriebsgewinn aus.

Ein weiteres Merkmal, für das vor allem bei Braunvieh aus Sicht des Zuchtziels ein wirtschaftliches Gewicht abgeleitet werden sollte, ist der Eiweißgehalt. Bereits beim wirtschaftlichen Gewicht für die Eiweißmenge wird deutlich, wie wichtig die Inhaltsstoffe der Milch für die Wirtschaftlichkeit des Betriebes sind. Allerdings kann man in einem Modell keine Gewichte für das Merkmal Eiweißmenge und Eiweißprozente bei gleichzeitiger Implementierung des Milchträgers ableiten, da dies einer Doppelberücksichtigung gleichkommt. Aus diesem Grund ist es in dieser Arbeit nicht möglich, eine Aussage über die Höhe des wirtschaftlichen Gewichtes für den Eiweißgehalt zu geben. Dies kann nur über Annahmen aus der Praxis getroffen werden.

Nach Mack et al. (1997) führt eine Verbesserung der Leistungsmerkmale zu einem starken Strukturwandel in der Milcherzeugung während eine Verbesserung der funk- 
tionale Merkmale vorrangig Kosteneinsparungen hervorruft. Es wird aber zukünftig in beiden Bereichen weiterhin zu steigenden Leistungen kommen. Nur bei den funktionalen Merkmalen und bei der Eiweißmenge sind Zuchtfortschritte uneingeschränkt wirtschaftlich. Nicht zu verhindern sind auch weiterhin Zuchtfortschritte beim Merkmal Fettmenge, was zwar züchterisch erwünscht ist, aber aufgrund agrarpolitischer Eingriffe in den Milch- und Rindfleischmarkt unter derzeitigen Bedingungen wirtschaftlich nicht positiv zu bewerten ist. Sollte es in Zukunft zu einer Milcherzeugung ohne Milchquotenbeschränkungen kommen, würde auch eine Leistungssteigerung für die Fettmenge unter wirtschaftlichen Gesichtspunkten wieder günstig sein, wie anhand der ermittelten Ergebnisse zu sehen ist.

\subsubsection{Funktionale Merkmale}

\section{Nutzungsdauer}

Nach Sölkner und Fürst (1998) kann die Verlängerung der Nutzungsdauer langfristig nur über die Einbeziehung von Merkmalen der Langlebigkeit in Zuchtprogramme vorgenommen werden. Es zeigt sich, dass diesem Merkmal die höchste wirtschaftliche Bedeutung zukommt. Eine verlängerte Nutzungsdauer bedingt eine Reduktion der anteiligen Aufzuchtkosten und die Ausnutzung der altersbedingten Steigerung der Milchleistung (Miesenberger, 1997). Es gibt zwei verschiedene Ansätze, das wirtschaftliche Gewicht für die Nutzungsdauer abzuleiten (Groen et al., 1997): Zum einen über eine Steigerung des Produktionsdauer und zum anderen über eine Reduzierung der unfreiwillige Merzungsrate. Es fällt ebenfalls auf, dass die wirtschaftliche Bedeutung der Nutzungsdauer nicht unabhängig vom vorgegebenen Populationsniveau gesehen werden darf (Mack et al., 1997). Wie in der vorliegenden Arbeit auch ist in der Holsteinpopulation das wirtschaftliche Gewicht höher als in der Fleckviehund Braunviehpopulation. Die durchschnittliche Nutzungsdauer in der Population ist bei Holstein geringer als in den anderen beiden Populationen, was das höhere wirtschaftliche Gewicht erklärt. Auch die Schlachtpreise nehmen Einfluss auf die Höhe der wirtschaftlichen Gewichte der Nutzungsdauer, wie anhand der eigenen Ergebnisse zu sehen ist. Daraus lässt sich folgern, dass bei einer geringen Nutzungsdauer eine Steigerung wirtschaftlich lohnender ist als bei schon hoher durchschnittlicher Nutzungsdauer. Dies zeigt auch Miesenberger (1997). Wünsch und Bergfeld (2001) stellen in ihrer Untersuchung fest, dass die Nutzungsdauer einen beträchtlichen Einfluss zeigt und fordern daher ein hohes wirtschaftliches Gewicht für dieses Merkmal. 
In Abb. 5.1 ist der Einfluss der durchschnittlichen Nutzungsdauer in der Herde auf das wirtschaftliche Gewicht der Nutzungsdauer bei Holstein anhand der eigenen Berechnungen dargestellt.

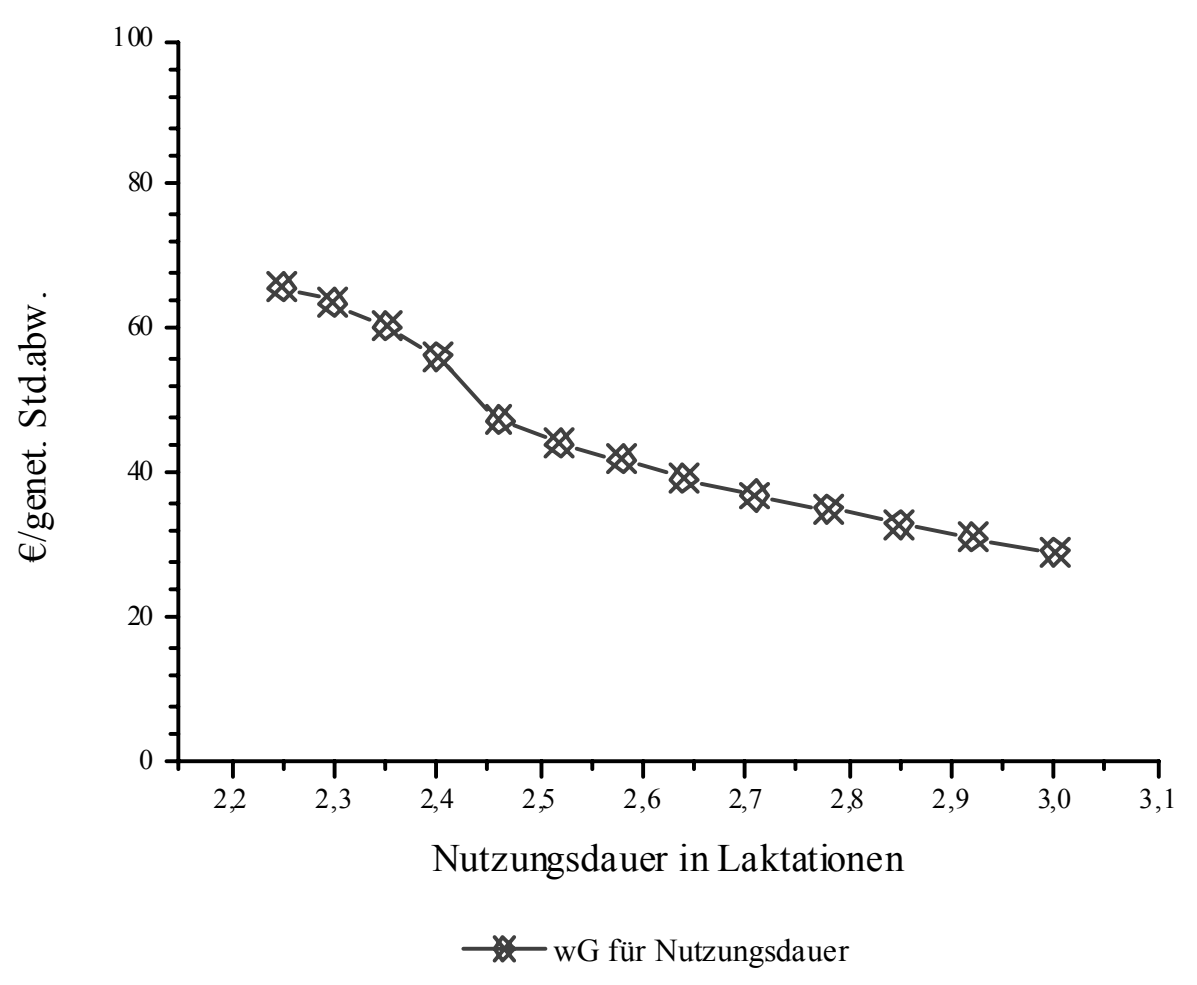

Abb. 5.1 Einfluss der durchschnittlichen Nutzungsdauer in der Milchviehherde auf das wirtschaftliche Gewicht der Nutzungsdauer bei Holstein

Es zeigt sich, wie auch von anderen Autoren beschrieben, eine starke Abhängigkeit des wirtschaftlichen Gewichts der Nutzungsdauer vom unterstellten Ausgangsniveau (Abb. 5.1). Sölkner und Fürst (1998) geben zu bedenken, dass eine entsprechende Berücksichtigung der Nutzungsdauer im Gesamtzuchtwert dazu beitragen sollte, die Fitness und Langlebigkeit der Kühe nicht weiter zu reduzieren. Dekkers (1991) unterstellt in seiner Basissituation eine durchschnittliche Nutzungsdauer in der Herde von 39,9 Monaten, die unter der durchschnittlichen Nutzungsdauer in den Niederlanden und den USA liegt. Des Weiteren zeigen sich deutliche Einflüsse eines suboptimalen Managements in Form von überhöhten Remontierungskosten auf die wirtschaftlichen Gewichte der unfreiwilligen Merzung. Nielsen (2004) gibt für die durchschnittliche Nutzungsdauer eine Länge von 2,6 Laktationen in Dänemark an. 
Das wirtschaftliche Gewicht ist für das Merkmal unfreiwillige Merzung abgeleitet und hat eine Höhe von $6,66 € \mathrm{je} \%$.

Bei Fleckvieh kommt es in den verschiedenen Szenarien zu einer Steigerung des wirtschaftlichen Gewichts für die Nutzungsdauer. Das höchste Gewicht liegt im Vergleichsszenario mit Milchquote vor. Im Gegensatz dazu stellt Nielsen (2004) heraus, dass es unter verringerter unfreiwilliger Merzung zu einem Anstieg der durchschnittlichen Milchleistung je Kuh und zu einer verlängerten durchschnittlichen Nutzungsdauer kommt. Daraus resultiert ein geringeres wirtschaftliches Gewicht für die unfreiwillige Merzung im Quotenszenario.

\section{Fruchtbarkeit}

Die geringe Gewichtung der Merkmale der Zuchtleistung erklärt Swalve (1998) durch die extrem niedrige Erblichkeit der Merkmale, da diese wenig Aussichten auf einen züchterische Verbesserung erkennen lassen. Demgegenüber steht das hohe wirtschaftliche Gewicht der Fruchtbarkeit bei der Rasse Holstein $\left(20 € / \sigma_{a}\right)$ in den eigenen Berechnungen. Mit einer Gewichtung des RZZ von 14\% im Gesamtzuchtwert würde dieser Merkmalskomplex eine deutliche Erhöhung der Bedeutung erfahren. Durch die unterstellten Annahmen in diesem Szenario zeigt sich, dass es durchaus wirtschaftlich sein kann, die Fruchtbarkeitsmerkmale zu verbessern. Unter Anbetracht der schlechten Fruchtbarkeitskennzahlen in der deutschen Holsteinpopulation erscheint dies durchaus sinnvoll. Im Gegensatz dazu steht das wirtschaftliche Gewicht von ca. $7 € / \sigma_{\mathrm{a}}$ bei Fleckvieh. Bei Braunvieh liegt das wirtschaftliche Gewicht mit etwa $14 € / \sigma_{\mathrm{a}} z w i s c h e n$ dem der anderen beiden Rassen. Vergleicht man die Konzeptionsraten aller Rassen, so fällt auf, dass Fleckvieh die höchste Konzeptionsraten hat, gefolgt von Braunvieh und Holstein. So sieht man hier die gleiche Tendenz wie bei den wirtschaftlichen Gewichten für das Merkmal Nutzungsdauer. Je höher das Merkmal im Referenzszenario angenommen ist, desto geringer ist der zusätzliche wirtschaftliche Nutzen bei einer Verbesserung dieses Merkmals. Der direkte Effekt einer verbesserten Konzeptionsrate ist eine verringerte Anzahl an Besamungen (Nielsen, 2004).

Amer et al. (1996) zeigen, dass die wirtschaftlichen Gewichte zur Verbesserung der Fruchtbarkeit der Kuh von den Annahmen bezüglich des phänotypischen Mittelwertes dieser Merkmale abhängen. Boichard (1990) stellt fest, dass die Fruchtbarkeit nach der Milchleistung das wirtschaftlich wichtigste Merkmal ist. Durch eine verschlechterte Fruchtbarkeit kommt es zu einer frühzeitigen Merzung der Kuh oder einer Verlängerung des Kalbeintervalls, was zusätzliche Kosten verursacht. Allerdings zeigt diese Untersuchung, dass der Milchpreis, die Herdenleistung und der Verkaufspreis für Kälber die Höhe des wirtschaftlichen Gewichtes für die Fruchtbar- 
keit nicht beeinflusst. Dem entgegen haben die Merzungsrate und die Remontierungskosten einen wichtigen Einfluss auf das wirtschaftliche Gewicht. Höhere Konzeptionsraten führen zu einer Steigerung des Gewinns (Dekkers, 1991). Einer der wichtigsten Gründe dafür sind gestiegene Einnahmen aus dem Milchverkauf, da sich das Kalbeintervall verkürzt. Im Vergleich zum Referenzszenario bei Holstein mit einem Grenznutzen von etwa $4 €$ je \% verbesserte Konzeptionsrate sind die Grenznutzen bei Dekkers (1991) im Basisszenario mit etwa 2,03€ je \% etwas geringer. Bei veränderter Basissituation werden jedoch 3,38€ je \% Mehrerlös erzielt. Vielfach werden andere Merkmale wie Kalbeintervall, Rastzeit, Besamungsindex und NonReturn-Rate zur Beschreibung der Fruchtbarkeit verwendet (Groen et al., 1997). Alle Merkmale werden direkt vom Besamungs- und Remontierungsmanagement des Landwirts beeinflusst. Durch eine Verschlechterung der Fruchtbarkeit kommt es zu erhöhten Besamungs- und Tierarztkosten, einer verlängerten Laktation und erhöhten Merzungsraten.

Allgemein auf die Merkmale der Zuchtleistung bezogen hat eine Erhöhung der Fruchtbarkeit in der Herde den höchsten wirtschaftlichen Nutzen je Merkmalseinheit (Mack et al., 1997). Die Zuchtleistungsmerkmale sind zudem stark von den Nutzungskosten für Arbeit abhängig. Sind die Nutzungskosten für Arbeit im Betrieb generell hoch, so steigt auch die wirtschaftliche Bedeutung einer Verbesserung der Nutzungsdauer, des Erstkalbealters und des Kalbeverlaufs.

\section{Kalbeverlauf}

Das wirtschaftliche Gewicht des Merkmals Kalbeverlauf liegt bei allen Rassen in der Höhe von $0,50 € / \sigma_{a}$ bis $2 € / \sigma_{a}$. Damit ist es das Merkmal mit der geringsten wirtschaftlichen Bedeutung. Allerdings muss dabei auch berücksichtigt werden, dass bei der Ableitung dieses Merkmals lediglich die direkten Einflussfaktoren implementiert sind, wie die Arbeitszeit und Entlohnung des Tierarztes sowie des Landwirtes. Allerdings werden die Folgen einer Geburt mit Hilfe oder einer Schwergeburt nicht im Modell mit einbezogen. Es ist jedoch offensichtlich, dass eine erschwerte Geburt Folgekosten durch eine Nachbehandlung sowie verlängere Kalbeintervalle und Verzögerungszeiten bedingt. Bekman und Van Arendonk (1993) implementieren Tierarztkosten, Arbeitszeit des Landwirts, Kälberverluste, reduzierte Milchleistung, verringerte Fruchtbarkeit und erhöhte Merzungsraten als Kostenfaktoren in ihr Modell. Alle anderen Parameter würden im vorliegenden Modell eine Doppelberücksichtigung bei der Ableitung der übrigen wirtschaftlichen Gewichte mit sich ziehen, was unter den gegebenen Vorraussetzungen zu vermeiden ist. 


\section{Totgeburtenrate}

Das wirtschaftliche Gewicht der Totgeburtenrate bewegt sich mit $20 € / \sigma_{\mathrm{a}}$ bei Fleckvieh und $16 € / \sigma_{\mathrm{a}}$ bei Braunvieh auf einem sehr hohen Niveau. Dagegen ist das wirtschaftliche Gewicht der Totgeburtenrate bei Holstein mit etwa $4 € / \sigma_{\mathrm{a}}$ relativ gering. Anhand der Untersuchungen bei Fleckvieh lässt sich feststellen, dass das wirtschaftliche Gewicht in hohem Maße von den im Referenzszenario verwendeten Preisen für Schlacht- und Verkaufstiere abhängig ist. Anhand des Schlachtpreises für Mastbullen wird deutlich, dass mit zunehmendem Preis auch das wirtschaftliche Gewicht der Totgeburtenrate ansteigt. Ähnliches ist bei den Parametern Schlacht- und Verkaufspreise für Kühe und Färsen sowie Verkaufspreise für Kälber zu beobachten. Da diese Parameter im Referenzszenario preislich sehr hoch angesetzt sind, folgt daraus auch das hohe berechnete wirtschaftliche Gewicht. So kann man schlussfolgern, dass es bei guten Schlachtpreisen noch wirtschaftlicher ist, geringere Kälberverluste zu haben, da der Gewinn vergleichsweise höher ist als bei einem schlechten Preisniveau. In Dänemark liegen die Grenznutzen für die Totgeburtenrate zwischen $-0,78 €$ je \% Totgeburten und $0,44 €$ je \% Totgeburten je nach unterstelltem Szenario (Nielsen, 2004). Eine verbesserte Totgeburtenrate bewirkt eine steigende Anzahl an zu vermarktenden Färsen und zu mästenden männlichen Kälbern. Auch Nielsen (2004) zeigt, dass die Totgeburtenrate mit der Bullenmast eng verknüpft ist. Zusätzlich ist es für den Betrieb günstig, die eigene Nachzucht stellen zu können und dementsprechend eine niedrige Totgeburtenrate zu haben.

\section{Persistenz}

Das Merkmal Persistenz ist eines der komplexesten Merkmale in der Rinderzucht. Da schon die Definition der Persistenz unterschiedlich ausgelegt wird (Dekkers et al., 1998; Swalve und Gengler, 1999) ist eine Ableitung des wirtschaftlichen Gewichtes ebenso unterschiedlich.

Höhere Milchleistungen sind mit längeren Kalbeintervallen verbunden. Dies ist nicht nur von biologischer Seite her relevant, sondern auch ökonomisch begründet, da der Hauptanteil des Einkommens aus dem Verkauf der Milch resultiert und nicht aus der Produktion von Kälbern (Swalve und Gengler, 1999). Bei Holstein hat die Persistenz ein relativ geringes wirtschaftliches Gewicht mit etwa $2 € / \sigma_{a}$. Bei einer Verbesserung des Merkmals, die in einer flacheren Laktationkurve resultiert, ermelkt eine Kuh im letzten Drittel der Laktation mehr Milch als zuvor, da die Milchleistung weniger stark abfällt. Zudem gehen die Kosten für die Zusatzfuttermittel zurück, da der Anstieg der Milchleistung moderater ausfällt und die Fütterung der Kuh weniger mit Kraftfutter ergänzt werden muss. Der zusätzliche Gewinn bei der Verbesserung des Merkmals lässt sich auf diese Komponenten zurückführen. 
Bei Fleckvieh liegt das wirtschaftliche Gewicht der Persistenz bei $14 € / \sigma_{\mathrm{a}}$ im Referenzszenario, was allerdings sehr hoch erscheint. Betrachtet man den Grenznutzen, so liegt dieser bei Fleckvieh bei ca. $6 €$ je Merkmalseinheit Persistenz. Es lässt sich erkennen, dass der Persistenz eine hohe genetische Standardabweichung in der Fleckviehpopulation unterstellt wird. Im Gegensatz dazu wird mit diesem Maß in der Zuchtwertschätzung bei Holstein nicht gearbeitet (Reinhardt, 2006). Daher kommt es zu diesen starken Unterschieden bei diesem Merkmal zwischen den Rassen. Wünsch und Bergfeld (2001) berechnen ein relatives wirtschaftliches Gewicht für die Persistenz in der sächsischen Holsteinpopulation von 5,1\% und schließen daraus, dass dieses Merkmal in das Zuchtziel aufgenommen werden sollte, da durch eine Verbesserung der Persistenz hohe Einsparungen im Bereich der Kraftfutterkosten gemacht werden können.

Es wird weiterhin diskutiert werden, wie und ob dieses Merkmal in die Zuchtwertschätzung implementiert werden soll. Bei Fleckvieh findet bereits eine Berücksichtigung im Gesamtzuchtwert statt, bei Holstein hingegen nicht.

\section{Somatic Cell Score (SCS)}

Der Somatic Cell Score (SCS) wird in der Zuchtwertschätzung als Hilfsmerkmal für die Zellzahl verwendet (VIT, 2006). Um eine angemessene Einbindung der Zellzahl in die Zuchtwertschätzung erzielen zu können, müssen die Daten normalverteilt sein. Da dies aber nicht der Fall ist, erfolgt eine Transformation der Zellzahl-Daten hin zum SCS.

Die Zellzahl bzw. SCS wird als wichtiges Merkmal gesehen, um Aussagen über die Eutergesundheit der Kuh geben zu können. Da ein gesundes Euter die Voraussetzung für eine hohe Milchproduktion bietet, ist es wichtig, dieses Merkmal entsprechend in der Zuchtwertschätzung zu berücksichtigen. Mit einem wirtschaftlichen Gewicht von $12 € / \sigma_{\mathrm{a}}$ bei Holstein und $21 € / \sigma_{\mathrm{a}}$ bei Fleckvieh und Braunvieh liegt dieses Merkmal im mittleren Bereich zwischen den Milchleistungsmerkmalen, der Nutzungsdauer und den übrigen funktionalen Merkmalen. De Jong und Harbers (2001) unterstellen in ihrer Untersuchung Kosten je Mastitisfall von etwa $81 €$ als Behandlungskosten und Ertragsausfall wegen nicht konsumierbarer Milch. Das wirtschaftliche Gewicht berechnen sie mit 27,17€/ $\sigma_{a}$. In der vorliegenden Arbeit sind die wirtschaftlichen Gewichte etwas geringer. 


\section{Melkbarkeit}

Das wirtschaftliche Gewicht der Melkbarkeit ist bei Holstein und Fleckvieh mit $1 €$ bis $2 € / \sigma_{\mathrm{a}}$ relativ gering. Etwas höher ist es bei Braunvieh mit $5 € / \sigma_{\mathrm{a}}$. Die Melkbarkeit ist dadurch gekennzeichnet, dass sie die ermolkenen Milchmenge je Minute ( $\mathrm{kg} / \mathrm{min})$ darstellt. Dies spiegelt zum einen die Schnelligkeit der Milchejektion des Tieres wider, zum anderen wird im Modell noch die Anzahl der Melkplätze mit in die Berechnung einbezogen. Es wird definiert, in welcher Zeit 100kg Milch gemolken werden können. Zudem geht die Arbeitszeit des Melkers dort mit ein, da natürlich in einem größeren Melkstand bei gleicher Anzahl Tiere effektiver gearbeitet werden kann.

Groen et al. (1997) zeigen, dass die meisten Autoren bei der Ableitung wirtschaftlicher Gewichte für das Merkmal Melkbarkeit die Kosten für die Arbeitszeit, die Elektrizität und die Ausführung des Melkstandes mit ins Modell mit einbeziehen. Das wirtschaftliche Gewicht für die Melkbarkeit wird überwiegend von der Höhe der Arbeitskosten und der Anzahl der Melkplätze im Melkstand beeinflusst. Wünsch und Bergfeld (2001) sehen die Notwendigkeit, die Melkbarkeit in das Zuchtziel zu integrieren, da vor allem in den Großbetrieben der Bedarf besteht, diese zu verbessern, da die eingesparte Arbeitszeit effektiver genutzt werden kann. Allerdings sollte auf die Wechselwirkungen mit der Eutergesundheit bei schnellem Milchfluss geachtet werden.

\section{Exterieur}

Für die Selektion von Bullen- und Kuhvätern sowie von Bullenmüttern werden in den Zuchtorganisationen überwiegend die geschätzten Zuchtwerte bezogen auf die Relativzuchtwerte Milch (RZM), Exterieur (RZE) und den Gesamtzuchtwert (RZG) verwendet (Swalve und Höver, 2003). In der Analyse der Beziehungen zwischen Zuchtwerten und Einsatzhäufigkeit der Bullen weisen Swalve und Höver (2003) darauf hin, dass bei den Landwirten das Exterieur einen überproportional hohen Stellenwert einnimmt. Bereits Schönmuth (1999) gibt zu Bedenken, dass die Geschichte der Schwarzbuntzucht in Deutschland von einem ständigen Kampf zwischen der Überbetonung des Exterieurs und der angemessenen Einbindung funktionaler Exterieurmerkmale geprägt ist. Nach Fürst-Waltl et al. (2004) gehen die Exterieurmerkmale indirekt über die Nutzungsdauer in den Gesamtzuchtwert ein. Sölkner et al. (2000) zeigen in ihren Berechnungen zum Gesamtzuchtwert bei Zweinutzungsrassen, dass die Landwirte bei subjektiver Zuteilung von wirtschaftlichen Gewichten, dem Exterieur einen Anteil von 15\% zuweisen würden. Allerdings zeigt die unabhängige Selektion auf Exterieur deutliche Auswirkungen auf den monetären Zuchtfortschritt. 
Mit zunehmendem relativem wirtschaftlichen Gewicht der Exterieurmerkmale sinkt der monetäre Gesamtfortschritt im Vergleich zur Ausgangssituation ohne Einbeziehung des Exterieurs. Dementsprechend erscheint das geringe wirtschaftliche Gewicht aus den eigenen Berechnungen nicht unrealistisch. Dies spiegeln die zuvor in anderen Untersuchungen gefundenen Ergebnisse wider. Swalve (1998) gibt zu bedenken, dass die hohe Gewichtung des Exterieurs in der Zuchtwertschätzung überraschen mag, allerdings verweist er auch auf die deutlichen Beziehungen zwischen vielen Merkmalen des Exterieurs und der Langlebigkeit. Da die Zuchtwerte für die Exterieurmerkmale mit einer ausreichenden Sicherheit früher geschätzt werden können als die der Langlebigkeit, eignet sich dies als Merkmal und lässt die hohe Gewichtung begründen. Edel und Dempfle (2004) versuchen anhand einer Contingent Value Studie das Zuchtziel vom Süddeutschen Kaltblut zu bestimmen. Dies ist eine weitere Möglichkeit, wirtschaftliche nicht direkt messbare Merkmale mit einem Grenznutzen zu belegen und die ökonomische Relevanz dieser Merkmale bei der Zuchtauswahl berücksichtigen zu können.

Fürst-Waltl et al. (2004) leiten wirtschaftliche Gewichte des Exterieurs für Fleckvieh und Braunvieh ab. Nach Berücksichtigung der Realisierungswahrscheinlichkeit kommen sie auf ein wirtschaftliches Gewicht je Herdendurchschnittskuh zwischen 6 und $10 €$ bei Fleckvieh bzw. 11 und $20 €$ bei Braunvieh. Damit würden die Exterieurmerkmale eine Gewichtung von 4 bis 7\% bei Fleckvieh und 7 bis 13\% bei Braunvieh erhalten. Allerdings ist eine Vergleichbarkeit mit anderen Merkmalen nicht gegeben. Mulder und Jansen (2001) zeigen eine deutliche Überlegenheit der wirtschaftlichen Gewichte der Milchleistungsmerkmale und der Nutzungsdauer gegenüber den Exterieurmerkmalen.

\subsubsection{Fleischleistungsmerkmale}

Die Fleischleistungsmerkmale verlieren im Gesamtzuchtwert bei Fleckvieh und Braunvieh zunehmend an Bedeutung. Allerdings ist es in Anbetracht des Zuchtziels beider Rassen nötig, die Fleischleistung auch im Gesamtzuchtwert zu berücksichtigen. Besonders in Zeiten hoher Fleischpreise kommt diesen Rassen die Berücksichtigung der Fleischleistungsmerkmale im Gesamtzuchtwert zugute. In der Untersuchung von Kaltenecker (1999) wird deutlich, dass die Bedeutung der Fleischleistungsmerkmale zurückgeht. Die führt er nicht nur auf die Regelungen durch die EUAgrarreform zurück, sondern auch auf eine ungünstige Nachfrageentwicklung des Rindfleischmarktes. 


\section{Nettozunahme}

Anstelle der täglichen Zunahme wird bei Fleckvieh und Braunvieh mittlerweile das Merkmal Nettozunahme im Zuchtwert berücksichtigt. Bei Fleckvieh liegt das wirtschaftliche Gewichte der Nettozunahme bei etwa $8 € / \sigma_{a}$, bei Braunvieh bei $6 € / \sigma_{\mathrm{a}} \mathrm{im}$ Referenzszenario. Die Quotensituation bzw. die Zusammensetzung des Milchauszahlungspreises übt dabei keinen Einfluss auf das wirtschaftliche Gewicht aus.

Durch die Verbesserung dieses Merkmals kann ein Mastbulle über kürzere Zeit im Betrieb eingestallt werden, da er mit einer höheren Nettozunahme auch eine kürzere Mastzeit benötigt. Dies führt dazu, dass eine höhere Umtriebsrate im Mastbereich erreicht werden kann und die Kosten pro Mastbulle etwas geringer werden.

\section{Handelsklasse}

Das wirtschaftliche Gewicht für die Handelsklasse wird ebenfalls für Fleckvieh und Braunvieh berechnet. Die Verbesserung der Handelsklasse um eine genetische Standardabweichung erzielt einen zusätzlichen Nutzen von ca. $3 €$ bei Fleckvieh sowie Braunvieh. Das Merkmal Handelsklasse ist insofern wichtig für die Fleischleistungsmerkmale, da dadurch die Qualität des Schlachtkörpers beschrieben wird und eine bessere Qualität automatisch auch einen höheren Erlös für den Betrieb bringt. Trotz des geringeren wirtschaftlichen Gewichtes der Handelsklasse gegenüber der Nettozunahme ist es im Sinne des Zuchtziels immer lohnend dieses Merkmal weiterhin zu verbessern.

\section{Ausschlachtung}

Das Merkmal Ausschlachtung wird in dieser Untersuchung ausschließlich bei Braunvieh untersucht. Durch eine verbesserte Ausschlachtung eines Mastbullen kann ein höherer Erlös je Schlachttier realisiert werden. Da das Merkmal relativ unkompliziert im Rahmen der Schlachtung ermittelt werden kann, liegt es nahe, dieses Merkmal entweder im Zuchtziel direkt oder als Hilfsmerkmal für die Zuchtwertschätzung zu berücksichtigen. Im Referenzszenario bei Braunvieh hat das Merkmal Ausschlachtung mit $11 € / \sigma_{\mathrm{a}}$ das höchste wirtschaftliche Gewicht unter den Fleischleistungsmerkmalen. 


\subsubsection{Weitere Merkmale}

Neben den in der Untersuchung berücksichtigten Merkmalen gibt es noch weitere Merkmale, für die wirtschaftliche Gewichte im Rahmen der Indexselektion abgeleitet werden können. Böbner (1994), Mack (1996) und Koenen et al. (1999; 2000) leiten Grenznutzen für das Merkmal Trockensubstanzaufnahme ab. Bei einer Verbesserung dieses Merkmals kommt es selbst bei deutlich ungünstigen wirtschaftlichen Rahmenbedingungen $\mathrm{zu}$ einer deutlichen Verbesserung der Wirtschaftlichkeit. Allerdings stellt sich die Frage, wie das Merkmal Trockensubstanzaufnahmevermögen in das Zuchtziel aufgenommen werden soll und auf welche Art und Weise eine Datenaufnahme erfolgen kann. Dies gestaltet sich sehr schwierig und scheint eine Leistungsprüfung nahezu unmöglich zu machen. Mithilfe eines Hilfsmerkmals wäre die Berücksichtigung im Selektionsindex bzw. in der Zuchtwertschätzung allerdings denkbar. Zusätzlich leiten Koenen et al. (1999; 2000) wirtschaftliche Gewichte für das Merkmal Körpergewicht ab.

Nielsen (2004) leitet neben den üblichen Merkmalen in der Zuchtwertschätzung wirtschaftliche Gewichte für die Merkmale Milchfiebererkrankung, Laminitiserkrankung und Körpergewicht ab. Anstelle der Milchleistungsmerkmale wird in der Untersuchung das wirtschaftliche Gewicht für das Merkmal Energiekorrigierte Milch (ECM) berechnet. Dabei sei darauf hingewiesen, dass in den Nordischen Ländern bereits seit längerem Gesundheitsmerkmale verstärkt im Zuchtziel vertreten sind und dementsprechend in die Zuchtwertschätzung einfließen.

\subsubsection{Zusammensetzung des Gesamtzuchtwertes}

Anhand der wirtschaftlichen Gewichte der einzelnen Merkmale kann auf die $\mathrm{Zu}-$ sammensetzung des Gesamtzuchtwertes geschlossen werden bzw. können Indexgewichte für die Indexnormalgleichung geschätzt werden. Bei Holstein wird der Ansatz gewählt, mithilfe der wirtschaftlichen Gewichte Indexgewichte zu schätzen. Bei Fleckvieh und Braunvieh wird eine einfache Approximation verwendet, um die Gewichtung der einzelnen Merkmale zueinander zu charakterisieren. Aufgrund unterschiedlicher Verfahren bei der Zuchtwertschätzung werden diese zwei Wege aufgezeigt (Fürst, 2006; Reinhardt, 2006). 
Es stellt sich die Frage, ob es eine Tendenz gibt, einen einzelbetrieblichen Nutzen einer bestimmten Rasse zu erkennen. Bestandteil der Untersuchung war es nicht, die Wirtschaftlichkeit einzelner Rinderrassen miteinander $\mathrm{zu}$ vergleichen. Allerdings zeigt bereits Kaltenecker (1999), dass milchbetonte Rassen von steigenden Milchpreisen und sinkenden Kraftfutterpreisen profitieren, während sich hohe Kälberpreise und die einzelbetriebliche Milchmengenkontingentierung vorteilhaft auf Zweinutzungsrassen auswirken.

\section{Holstein}

Bei Holstein wird sehr schnell deutlich, dass sich unter Einbeziehung aller Merkmalskomplexe in den Gesamtzuchtwert die Konstellation dieser zueinander verändert. Nach wie vor behalten die Milchleistungsmerkmale einen hohen Anteil an den relativen wirtschaftlichen Gewichten mit 58\%. Dahingegen verliert der Merkmalskomplex Nutzungsdauer und Exterieur massiv. Ein leichter Anstieg ist beim Komplex der Zellzahl und vor allem der Zuchtleistung festzustellen. Pieters et al. (1997) zeigen in ihren Sensitivitätsanalysen, dass die durchschnittlichen Herdenleistungen der Fettmenge und vor allem der Eiweißmenge die relativen wirtschaftlichen Gewichte am meisten beeinflussen.

Würde man diesen Ansatz bei der Umsetzung des Gesamtzuchtwertes verfolgen, hätte man kaum noch Gewicht auf den Merkmalen des Exterieurs. Aus Sicht der Züchterschaft ist allerdings das Exterieur eines der wichtigsten Merkmale (Swalve und Höver, 2003). Da die Exterieurmerkmale auch eine wichtige Rolle bei der Schätzung des Relativzuchtwertes für Nutzungsdauer spielen, erscheint diese Variante als nicht durchsetzbar. Es muss allerdings berücksichtigt werden, dass gerade die Exterieurmerkmale ihre entscheidende Aufgabe im Bereich der Hilfsmerkmale haben. Da diese Merkmale bereits bei der Ableitung wirtschaftlicher Gewichte als eigenes Merkmal in die Berechnungen mit einfließen, würde eine Berücksichtigung bei der Schätzung der Gewichte des Exterieurs zu einer Doppelberücksichtigung führen (Fürst-Waltl et al., 2004; Krogmeier et al., 2006; Schierenbeck, 2006).

Für die weitere züchterische Entscheidung beim Merkmalskomplex Zuchtleistung scheint es durchaus wichtig zu sein, diesen Komplex noch stärker in den Gesamtzuchtwert zu integrieren. Gerade im Bereich Fruchtbarkeit stößt man in der Population immer wieder an Grenzen. Die Remontierungsraten steigen, es müssen Färsen zugekauft werden, um die eigene Herde remontieren zu können und die Besamungserfolge verschlechtern sich. Allerdings besitzen alle Zuchtleistungsmerkmale eine geringe Heritabilität, so dass es schwierig ist, diese konsequent in die Selektion einzubeziehen. Swalve (1998) gibt dies zu bedenken, verweist aber gleichzeitig darauf, dass es wichtig ist, die Zuchtleistung im Gesamtzuchtwert zu halten. Für die kom- 
menden Jahre sollen im Bereich der Zuchtleistung Merkmale ausgetauscht werden und die Datengrundlage verbessert werden. Dies würde schon einen entscheidenden Schritt in die Richtung einer besseren züchterischen Ausnutzung dieser Merkmale mit sich bringen.

De Jong und Harbers (2001) untersuchen die Effekte einer verstärkten Gewichtung der funktionalen Merkmale im Gesamtzuchtwert in den Niederlanden. Es zeigt sich, dass unter den angenommenen Bedingungen die relative Gewichtung des Merkmalskomplexes Milchleistung bei 57\% liegt und die der Langlebigkeit und der Gesundheitsmerkmale bei 43\%. Unterteilt man die funktionalen Merkmale weiter, so würde die Eutergesundheit einen Anteil von $11 \%$ an der Gewichtung erhalten, die Fruchtbarkeit einen Anteil von 7\%. Die Kalbemerkmale gehen dann mit 4\% und 7\% in den Gesamtzuchtwert ein. Pärna et al. (2003) vergleichen die Gesamtzuchtwerte von elf verschiedenen europäischen Holstein-Ländern. In allen Ländern erhält die Eiweißmenge mehr Gewicht als die Fettmenge und es wird gegen die Milchleistung und zugunsten der funktionalen Merkmale selektiert. Wünsch und Bergfeld (2001) stellen fest, dass die Zuchtleistungsmerkmale Konzeptionsrate, Kalbeverlauf und Totgeburtenrate ein Gewicht zwischen $4 \%$ und $5 \%$ besitzen.

\section{Fleckvieh}

Bisher hatte der Gesamtzuchtwert bei Fleckvieh eine Gewichtung der Komplexe Milch:Fleisch:Fitness von 39:17:44. Damit waren die funktionalen Merkmale am stärksten gewichtet. Betrachtet man die neuen Berechnungen, so fällt auf, dass die Milchleistungsmerkmale und funktionalen Merkmale an Bedeutung gewinnen, was zu Lasten der Fleischleistungsmerkmale geht. Im Vergleichsszenario würde die Gewichtung noch deutlicher hin zu den Milchleistungsmerkmalen tendieren, was sich durch den Milchauszahlungspreis und damit das hohe wirtschaftliche Gewicht der Eiweißmenge begründen lässt. $\mathrm{Zu}$ einem dramatischen Rückgang bei der Gewichtung der Milchleistungsmerkmale kommt es, wenn man das Vergleichsszenario unter Milchquotenbedingungen betrachtet. Durch das negative wirtschaftliche Gewicht der Fettmenge kommt es zu diesem Rückgang, die Fitnessmerkmale gewinnen unter diesem Szenario an Bedeutung.

Mack et al. (1997) stellen fest, dass sich die Grenznutzen zwischen Fleckvieh und Holstein in ihren Untersuchungen nur geringfügig voneinander unterscheiden. Es zeigt sich, dass unter den unterstellten Bedingungen die Grenznutzen der Milchleistungsmerkmale bei Holstein niedriger sind als bei Fleckvieh, da bei Holstein die Grenzkosten einer Leistungssteigerung höher sind. Vergleicht man in den vorliegenden Berechnungen die wirtschaftlichen Gewichte der Milchleistungsmerkmale bei Holstein im Referenzszenario und Fleckvieh im Vergleichsszenario ohne Quote, so 
fällt auf, dass die Gewichtung der Merkmale Fett- und Eiweißmenge bei Holstein stärker gespreizt ist. Dies ergibt sich aus der Annahme des unterschiedlichen Milchauszahlungspreises in Norddeutschland und in Bayern. Bei der relativen Gewichtung der Merkmale haben die Milchleistungsmerkmale bei Holstein mit 58\% einen wesentlich bedeutenderen Einfluss als bei Fleckvieh mit 46\%. Allerdings gehen bei Fleckvieh zusätzlich noch die Fleischleistungsmerkmale in den Gesamtzuchtwert mit ein. Trotz allem nimmt bei beiden Rassen die Bedeutung der Milchleistungsmerkmale zu. Miesenberger et al. (1997) stellen fest, dass die wirtschaftlichen Gewichte für den Kalbeverlauf und die Totgeburtenrate niedriger sind als für die Merkmale Fruchtbarkeit und Nutzungsdauer. Aus der zusätzlichen Berechnung von Indexgewichten und Selektionserfolgen zeigt sich, dass es nötig ist, die funktionalen Merkmale zusätzlich im Gesamtzuchtwert zu berücksichtigen.

\section{Braunvieh}

Die Zusammensetzung des Gesamtzuchtwertes bei Braunvieh setzt sich aus $48 \%$ Milchleistungsmerkmalen, 5\% Fleischleistungsmerkmalen und 47\% funktionalen Merkmalen zusammen. Betrachtet man das Referenzszenario und das Vergleichsszenario, sieht man, dass sich die Gewichtung der Milchleistung nicht bedeutend verändert. Dafür erhöht sich der Anteil der Fleischleistungsmerkmale von 5\% auf 10\%. Zusätzlich erhält das Exterieur einen Anteil von 1\% am Gesamtzuchtwert. So bleibt festzustellen, dass auch unter zukünftigen Bedingungen der Gesamtzuchtwert für Braunvieh keine bedeutenden Veränderungen erfährt.

\subsection{Indexgewichte}

Die Ableitung von Indexgewichten erfordert Kenntnisse über die Ökonomie der einbezogenen Merkmale und die phänotypischen und genetischen Parameter (Pärna et al., 2003). In der Indexselektion ist das Zuchtziel als lineare Funktion aller wirtschaftlich wichtigen Merkmale definiert. Den größten Verlust beim Selektionserfolg erhält man dann, wenn man wirtschaftliche Gewichte verwendet, die zum Zeitpunkt der Selektion der Tiere berechnet worden sind und nicht für den Zeitpunkt, wenn der genetische Fortschritt zum Tragen kommt (Pieters et al., 1997). Selektion ist das wesentliche Mittel mit dem ein Tierzüchter eine gerichtete genetische Veränderung in der Population erzielen kann (Melton et al., 1979). Diese ist dazu da, eine Verbesserung der Merkmale in eine bestimmt Richtung zu erreichen. 
Für eine Gesamtrangierung aller züchterisch und wirtschaftlich relevanten Merkmale ist der Gesamtzuchtwert eine optimale Leitfunktion (Reinhardt, 1998). In diesem Zusammenhang ist auch die Berechnung und Anwendung des Gesamtzuchtwertes zu verstehen. Durch die Gesamtrangierung und die Berücksichtigung aller Zuchtzielmerkmale in einem Komplex können Mängel in den einen Merkmalen durch Vorzüge in anderen Merkmalen kompensiert werden. Damit kann in der gesamten Zuchtpopulation ein Zuchtfortschritt erreicht werden, ohne dass bestimmte Merkmale durch Nichtberücksichtigung einen negativen Zuchtfortschritt realisieren.

Im Wesentlichen wird die Indexselektion durch die wirtschaftlichen Gewichte und die Heritabilitäten der Merkmale beeinflusst (Smith, 1983). Gerade Merkmale mit hohen wirtschaftlichen Gewichten neigen dazu den Index zu dominieren, während andere Merkmale von geringerer Bedeutung sind. Es ist allerdings wichtig, das Zuchtziel konkret zu definieren, damit alle Merkmale berücksichtigt werden können, die von hohem wirtschaftlichen Interesse und Bedeutung sind. Dies ist auch in der vorliegenden Arbeit zu beobachten. Nach Gibson (1989b) sollte das Ziel der Selektion in der Steigerung der ökonomischen Effizienz liegen und nicht dazu dienen, Unzulänglichkeiten im Management zu korrigieren.

Für das zukünftig ausgerichtete Zuchtziel ist es nötig, sowohl neue Merkmale in das Zuchtziel mit aufzunehmen als auch unpassende Merkmale zu entfernen oder neu zu überarbeiten (Groen et al., 1997). Dies gilt ebenso für die Informationsmerkmale im Selektionsindex. Zum einen ergeben sich immer neue Methoden der Leistungsprüfung und weiterhin wird diese konstant optimiert und verbessert. Daher scheint es nötig, auch das Zuchtziel und den Selektionsindex entsprechend regelmäßig zu aktualisieren. Wie anhand der eigenen Berechnungen zu sehen ist, nehmen die unterstellten wirtschaftlichen Gewichte hohen Einfluss auf die b-Werte, allerdings unterliegen die genetischen Parameter auch immer wieder neuen Schätzungen. Zunehmend an Bedeutung in der Zuchtwertschätzung und bei der Ausrichtung des Zuchtziels nehmen die Merkmale, die mit der Umweltwirkung und der Tiergerechtheit in Zusammenhang stehen (Groen et al., 1997). Zudem werden die Merkmale, die einen Einfluss auf die Milchqualität nehmen wie der Proteingehalt und der SCS, immer wichtiger. Generell erscheint es wichtig, die Qualitätsparameter, auch bei der Fleischleistung, mit in das Zuchtziel zu implementieren. 
Dempfle (1992) gibt zu bedenken, dass es bei hohen Grenznutzen für die Milchleistungsmerkmale und den vorhandenen niedrigen Heritabilitäten für die funktionalen Merkmale durch die Berücksichtigung dieser Merkmale nur zu einer geringen Steigerung des Züchtungserfolges kommt. Daher sollte der Schätzung der genetischen $\mathrm{Pa}$ rameter auch weiterhin entsprechende Aufmerksamkeit geschenkt werden, um den Zuchtfortschritt kontinuierlich steigern zu können. Allerdings muss dabei auch an die Bereitstellung der Daten durch die Landwirte gedacht werden, die bei weitem noch nicht routinemäßig erfolgt und optimiert werden sollte. 


\section{Schlussfolgerung}

Der Gesamtzuchtwert fasst die einzelnen Merkmale im Zuchtziel entsprechend ihrer ökonomischen Bedeutung und ihrer genetischen Beziehungen zusammen (Hazel und Lush, 1942). Dabei wird bei der Selektion nach dem Gesamtzuchtwert der größtmögliche monetäre Zuchtfortschritt realisiert. Um einen Index konzipieren zu können, werden für die Teilzuchtwerte Indexgewichte mit Hilfe von wirtschaftlichen Gewichten und genetischen Parametern berechnet und anschließend ein Gesamtzuchtwert ausgegeben.

Vorrangiges Ziel der Arbeit ist die Ableitung wirtschaftlicher Gewichte unter der Berücksichtigung zukünftiger Rahmenbedingungen. Da die europäische Landwirtschaft unter großem Außenhandelsdruck steht und sich die politische Situation sowie die Marktpreise kontinuierlich verändern, ist es für die Züchtung nötig, sich an die Strukturen anzupassen und ihr Zuchtprogramm an zukünftigen Marktsituationen auszurichten, um mit den produzierten Tieren wirtschaftlich handeln zu können. Deshalb muss auf den Wandel in der Quotenpolitik reagiert werden und das Ziel der Zuchtunternehmen muss es sein, die Wirtschaftlichkeit der einzelnen Merkmale im Zuchtziel zu hinterfragen und die Ökonomie dieser auch in Zukunft zu kennen bzw. zu analysieren. Ein entscheidendes Kriterium ist dabei die Senkung der Kosten in der Tierproduktion, da sich eine Erhöhung der Einkünfte immer schwieriger gestaltet. Auch muss darauf geachtet werden, dass sich die Rasse, besonders die Rassen Fleckvieh und Braunvieh, in der nationalen und internationalen Konkurrenz bewehren können und weiterhin eine wirtschaftliche Milchproduktion betrieben werden kann. Dementsprechend sollten sich die Rasseprofile von anderen Rassen abheben und die eigenen Stärken herausstellen. Bei der Rasse Fleckvieh sollte im Zuchtziel und auch im Zuchtprogramm weiterhin die Fleischleistung mit berücksichtigt werden, die Rasse Braunvieh ordnet sich zwischen Fleckvieh und Holstein ein und setzt auf gute funktionale Merkmale und hohe Eiweißgehalte in der Milch.

Es zeigt sich, dass die wirtschaftlichen Gewichte maßgeblich durch die Annahme des unterstellten Szenarios und der Markt- und Preissituationen beeinflusst werden. Daher sind die Annahmen der zukünftigen Produktionsbedingungen gründlich zu hinterfragen und korrekt zu analysieren. Dadurch kann eine Über- bzw. Unterschätzung der einzelnen wirtschaftlichen Gewichte vermieden werden (Nielsen, 2004). 
Wie aus der vorliegenden Arbeit ersichtlich wird, nehmen die Milchleistungsmerkmale eine herausragende Stellung bei der Wirtschaftlichkeit des Betriebs ein. Sollte es zum Auslaufen der Quotenbedingungen in Europa kommen, so wie es derzeit geplant ist, nimmt der Anteil der Milchleistungsmerkmale an den relativen wirtschaftlichen Gewichten, besonders bei Holstein und Fleckvieh, weiterhin zu. Auch wenn der relative Anteil der wirtschaftlichen Gewichte der funktionalen Merkmale geringer ist, sollten diese trotzdem in ausreichendem Maße in der Zuchtwertschätzung berücksichtigt werden. Zwar kommt es bei vielen dieser Merkmale in der Berechnung des Zuchtfortschritts durch die geringen Heritabilitäten zu keiner umfassenden Steigerung, allerdings muss verhindert werden, dass sich ein negativer genetischer Trend einstellt. Deshalb ist eine Einbeziehung dieser Merkmale unbedingt von Nöten.

Die Untersuchungen sollen als Grundlage dienen, um die Zuchtzielentscheidung der Zuchtunternehmen zu unterstützen und aufzeigen, welchen Einfluss die veränderten Rahmenbedingungen auf die Zusammensetzung des Gesamtzuchtwertes und damit auf die Wirtschaftlichkeit der Züchtung haben. 


\section{$7 \quad$ Zusammenfassung}

In der vorliegenden Arbeit werden im Rahmen eines Projektes der Organisationen Deutscher Holstein Verband e.V. und der Arbeitsgemeinschaft Süddeutscher Rinderzüchter e.V. für die Rassen Holstein, Fleckvieh und Braunvieh wirtschaftliche Gewichte sowie optimale Indexgewichte für den Gesamtzuchtwert abgeleitet.

Da die Zuchtwertschätzung für die Rassen Fleckvieh und Braunvieh von den Ländern Deutschland und Österreich gemeinsam durchgeführt wird, erfolgt auch die Berechnung der wirtschaftlichen Gewichte für beide Länder. Die Berechnungen erfolgen unter Berücksichtigung der aktuellen wirtschaftlichen und politischen Rahmenbedingungen und bieten damit die Möglichkeit, das Zuchtziel an zukünftige Marktbedingungen anzupassen und eine wirtschaftliche Milch- bzw. Zweinutzungskuh zu züchten.

Die Ableitung der wirtschaftlichen Gewichte erfolgt mit Hilfe des AmerMiesenberger-Programms (Amer et al., 1994; Miesenberger, 1997). Dabei handelt es sich um ein Herdenmodell auf Betriebsbasis, welches die Teilbereiche Milchviehherde, Jungviehaufzucht und Bullenmast berücksichtigt. Die in das Modell eingehenden Herdenparameter sowie Kosten und Erlöse des Betriebes sind in Anlehnung an die Betriebszweigauswertungen der Länder Schleswig-Holstein und Bayern ausgewählt und sollen nach Möglichkeit den Durchschnittsbetrieb der einzelnen Rasse widerspiegeln.

Darüber hinaus wurde eine Methode entwickelt, um die Unsicherheiten der zukünftigen Marktsituationen zu berücksichtigen und eine größere Variabilität in der Produktion in die Untersuchung einbeziehen zu können. Die Berechnungen erfolgen jeweils für ein Szenario mit und ohne Milchquotenbeschränkungen. Bei den Rassen Fleckvieh und Braunvieh werden darüber hinaus zwei verschiedene Arten der Zusammensetzung des Milchauszahlungspreises analysiert und die Auswirkungen eines gespreizten Fett- und Eiweißauszahlungspreises untersucht. Abschließend werden die Einflüsse veränderter Herden- und Preisparameter auf die wirtschaftlichen Gewichte einzelner Merkmale aufgezeigt.

Für die Rasse Holstein werden, basierend auf den abgeleiteten wirtschaftlichen Gewichten, optimale Indexgewichte für den Gesamtzuchtwert berechnet. Ein von Reinhardt (2006) verwendetes Indexprogramm zur Berechnung des Gesamtzuchtwertes dient dabei als Grundlage. Durch die Berücksichtigung der wirtschaftlichen Gewichte und der genetischen Beziehungen zwischen den Merkmalskomplexen werden die 
Indexgewichte geschätzt und der Einfluss der wirtschaftlichen Gewichte und unterschiedlichen Teilzuchtwerte auf den Gesamtzuchtwert analysiert.

Es wird deutlich, dass die wirtschaftlichen Gewichte maßgeblich durch die Annahme des Szenarios und die unterstellten Herdenparameter, die eine Rasse charakterisieren, beeinflusst werden.

Bei der Rasse Holstein zeigt sich, dass die Veränderung der wirtschaftlichen Gewichte maßgeblich durch die Annahme des Szenarios ohne Milchquotenbeschränkungen beeinflusst wird. Die wesentlichen Veränderungen gegenüber dem derzeitigen Gesamtzuchtwert sind eine höhere Gewichtung der Leistungsmerkmale, eine deutlich höhere Gewichtung der Fruchtbarkeit, eine geringere Gewichtung der Nutzungsdauer sowie ein geringes wirtschaftlich begründetes Gewicht für den Merkmalskomplex Exterieur. Auf der Basis der Ergebnisse von Schierenbeck (2006) kann eine höhere Gewichtung des Exterieurs gewählt werden. Die Anteile der einzelnen Teilzuchtwerte am Gesamtzuchtwert (RZG) bei der Rasse Holstein sind in der Höhe von 58\% RZM, 1\% RZE, 7\% RZS, 20\% RZN und 14\% RZZ. Unter Berücksichtigung der Ergebnisse von Schierenbeck (2006) wird eine Verteilung der Teilzuchtwerte mit 54\% RZM, 9\% RZE, 6\% RZS, 18\% RZN und 13\% RZZ vorgeschlagen.

Bei Fleckvieh kommt es durch den Wegfall der Milchquote zu einem erhöhten wirtschaftlichen Nutzen bei einer Leistungssteigerung der Milchleistungsmerkmale. Eine starke Spreizung des Auszahlungspreises für die Komponenten Fett- und Eiweißmenge führt dazu, dass die Eiweißmenge das dominierende wertbestimmende Merkmal ist. Bei den Fitnessmerkmalen zeigt der Kalbeverlauf ein geringes wirtschaftliches Gewicht, wohingegen die Totgeburtenrate, beeinflusst durch hohe Schlacht- und Verkaufspreise bei Kühen, Färsen, Kälbern und Mastbullen, einen hohen wirtschaftlichen Nutzen hat. Die Fleischleistungsmerkmale haben im Vergleich $\mathrm{zu}$ den anderen Merkmalskomplexen einen geringen wirtschaftlichen Nutzen. Allerdings führt auch die Nichtberücksichtigung der Merkmale Ausschlachtung bzw. Fleischanteil dazu, dass sich der Anteil dieses Komplexes am Gesamtzuchtwert reduziert, da diese Merkmale nicht mehr routinemäßig in der Leistungsprüfung erfasst werden. Der Gesamtzuchtwert (GZW) im Referenzszenario setzt sich aus 49\% Milchleistungsmerkmalen, 5\% Fleischleistungsmerkmalen und 46\% Fitnessmerkmalen zusammen.

Bei der Rasse Braunvieh zeigen sich keine bedeutenden Veränderungen in der Gewichtung der Merkmalskomplexe aufgrund des angenommenen Auslaufens der Quotenregelung. Der Anteil der Milchleistungsmerkmale am Gesamtzuchtwert geht leicht zurück, während der Anteil der Fitnessmerkmale nahezu gleich bleibend ist. Allerdings erhöht sich der Anteil der Fleischleistungsmerkmale, begründet durch die Annahme hoher Schlacht- und Verkaufspreise bei Fleisch. Das Exterieur hat auch bei 
Braunvieh, wie bei Holstein, eine untergeordnete wirtschaftliche Bedeutung. Im Gesamtzuchtwert (GZW) im Referenzszenario haben die Milchleistungsmerkmalen einen Anteil von 47\%, die Fleischleistungsmerkmale 5\%, die Fitnessmerkmale 42\% und das Exterieur 1\%.

Anhand der Untersuchungen wird deutlich, dass die wirtschaftlichen Gewichte für die Zuchtwertschätzung unter veränderten politischen und wirtschaftlichen Rahmenbedingungen immer wieder neu zu berechnen sind, da nur unter der Einbeziehung der realen Annahmen auch zielgerichtet gezüchtet werden kann. 


\section{$8 \quad$ Literaturverzeichnis}

Albera, A., P. Carnier und A. F. Groen (2004).

Definition of a breeding goal for the Piemontese breed: economic and biological values and their sensitivity to prduction circumstances. Livest. Prod. Sci. 89: 67-78.

ALI, T. E. und L. R. SCHAEFFER (1987).

Accounting for covariances among test day milk yields in dairy cows. Can. J. Anim. Sci. 67: 637-644.

AMER, P. R. und G. C. Fox (1992).

Estimation of economic weights in genetic improvement using neoclassical production theory: an alternative to rescaling. Anim. Prod. 54: 341-350.

AMER, P. R., G. C. FoX und C. SMITH (1994).

Economic weights from profit equations: appraising their accuracy in the long run. Anim. Prod. 58: 11-18.

AMER, P. R., B. G. LOWMAN und G. SimM (1996).

Economic values for reproduction traits in beef suckler herds based on a calving distribution model. Livest. Prod. Sci. 46: 85-96.

BAFF (2006).

Persönliche Mitteilungen. 2. Mai 2006. Kulmbach. Bundesforschungsanstalt für Ernährung und Lebensmittel.

BAUMUNG, R. und J. SöLKNER. Universität für Bodenkultur (1998).

Erstellung eines ökonomischen Gesamtzuchtwertes für die Rassen Simmentaler Fleckvieh, Schweizer Braunvieh und Holstein Friesian - Abschlussbericht. Wien.

\section{BEKMAN, H. und J. A. M. VAN ARENDONK (1993).}

Derivation of economic values for veal, beef and milk production traits using profit equations. Livest. Prod. Sci. 34: 35-56.

BLAAS, K. und J. SÖLKNER (1996).

Zuchtwertschätzung für Fleischleistungsmerkmale beim Zweinutzungsrind. Seminar des genetischen Ausschusses der ZAR, Salzburg, Zentrale Arbeitsgemeinschaft österreichischer Rinderzüchter. 34-39. 
BöBNER, C. (1994).

Schätzung wirtschaftlicher Gewichte für sekundäre Leistungsmerkmale bei Schweizerischen Zweinutzungsrindern unter Anwendung der dynamischen Optimierung. Eidgenössische Technische Hochschule. Zürich, Schweiz. Diss.

BOICHARD, D. (1990).

Estimation of the economic value of conception rate in dairy cattle. Livest. Prod. Sci. 24: 187-204.

BRASCAMP, E. W. (1984).

Selection indices with constraints. Anim. Breed. Abstr. 52(9): 645-654.

BRASCAMP, E. W., C. SMITH und D. R. GUY (1985).

Derivation of economic weights from profit equations. Anim. Prod. 40: 175180.

DE JoNG, G. und A. G. F. HARBERS (2001).

The effect of more health traits in DPS on economic selection. Proceeding of the 2001 Interbull Meeting, Budapest, Hungary, Interbull Bulletin. no. 27. 97101.

DEKKERS, J. C. M. (1991).

Estimation of economic values for dairy cattle breeding goals: bias due to sub-optimal management policies. Livest. Prod. Sci. 29: 131-149.

\section{DeKKers, J. C. M., J. H. TEN HAG und A. WeERSinK (1998).}

Economic aspects of persistency of lactation in dairy cattle. Livest. Prod. Sci. 53: $237-252$.

DEMPFLE, L. (1992).

Berücksichtigung von Fruchtbarkeit und Eutergesundheit in der Rinderzüchtung. Züchtungskunde 64(6): 447-457.

DODENHOFF, J. (2005).

Persönliche Mitteilung. 1. September 2005.

DODENHOFF, J. (2006).

Persönliche Mitteilung. 1. Dezember 2006.

EDEL, C. und L. DEMPFLE (2004).

Ergebnisse einer Contingent Valuation Studie zur Bestimmung des Zuchtziels beim Süddeutschen Kaltblut. Züchtungskunde 76: 108-116. 
FEWSON, D. (1967).

Rentable Veredlungswirtschaft - tierzüchterische Möglichkeiten. Züchtungskunde 39: 408-431.

FITZHUGH, H. A. (1976).

Analysis of growth curves and strategies for altering their shape. J. Anim. Sci. 42: 1036-1051.

FürSt-WALTL, B., J. WIESER, C. FÜRST und J. SölKNER (2004).

Einfluss des Exterieurs auf den Versteigerungspreis von Fleckvieh- und Braunvieh-Kalbinnen. Züchtungskunde 76(3): 149-161.

FÜRST, C. (1999).

Gesamtzuchtwert im internationalen Vergleich. Seminar des genetischen Ausschusses der ZAR, Salzburg, Zentrale Arbeitsgemeinschaft österreichischer Rinderzüchter. 27-34.

FÜRST, C. (2006).

Persönliche Mitteilung. 13. Januar 2006.

FüRST, C. und B. GREDLER (2006).

Züchterische Aspekte der Fruchtbarkeit. Seminar des Ausschusses für Genetik der ZAR, Zentrale Arbeitsgemeinschaft österreichischer Rinderzüchter. $31-48$.

FüRST, C. und D. KROGMEIER (2004).

Das Exterieur in der Zuchtwertschätzung. Seminar des genetischen Ausschusses der ZAR, Salzburg, Zentrale Arbeitsgemeinschaft österreichischer Rinderzüchter. 29-45.

GIBSON, J. P. (1989a).

Economic weights and index selection of milk production traits when multiple production quotas apply. Anim. Prod. 49: 171-181.

GiBSON, J. P. (1989b).

Selection on the major components of milk: alternative methods of deriving economic weights. J. Dairy Sci. 72: 3176-3189.

\section{GiBSON, J. P. und J. W. WiLton (1998).}

Defining multiple-trait objectives for sustainable genetic improvement. J. Anim. Sci. 76: 2303-2307. 
GREDLER, B. (2004).

Einfluss wirtschaftlicher Gewichte und genetischer Parameter auf den Zuchtfortschritt der Zuchtprogramme Fleckvieh Austria und Braunvieh Austria. Universität für Bodenkultur. Wien, Österreich. Diplomarbeit.

Groen, A. F. (1989a).

Cattle breeding goals and production circumstances. Agricultural University. Wageningen, Netherlands. Ph. D.

GroEN, A. F. (1989b).

Economic values in cattle breeding. I. Influences of production circumstances in situations without output limitations. Livest. Prod. Sci. 22: 1-16.

Groen, A. F., T. Steine, J. J. Colleau, J. Pedersen, J. Pribyl und N. Reinsch (1997).

Economic values in dairy cattle breeding, with special reference to functinal traits. Report of an EAAP-working group. Livest. Prod. Sci. 49: 1-21.

Gruber, L., F. J. SCHWARZ, D. ERdin, B. FISCHER, H. SPIEKERS, H. STEINGAß, U. Meyer, A. Chassot, T. Jilg, A. Obermaier und T. Guggenberger (2004).

Vorhersage der Futteraufnahme von Milchkühen - Datenbasis von 10 Forschungs- und Universitätsinstituten Deutschlands, Österreichs und der Schweiz. VDLUFA-Schriftenreihe. Band 60 - Kongressband 2004.

GuO, Z. (1998).

Modelle zur Beschreibung der Laktationskurve des Milchrindes und ihre Verwendung in Modellen zur Zuchtwertschätzung. Georg-AugustUniversität. Göttingen, Deutschland. Diss.

HABIER, D., K.-U. GöTZ und L. DEMPFLE (2004).

Ökonomische Gewichte von Leistungsmerkmalen für Vaterrassen in der bayerischen Schweinezucht. Züchtungskunde 76(5): 307-320.

HARRIS, B. L. und A. E. FreEMAN (1993).

Economic weights for milk yield traits and herd life under various economic conditions and production quotas. J. Dairy Sci. 76: 868-879.

HAZEL, L. N. (1943).

The genetic basis for constructing selection indexes. Genetics 28: 476-490.

HAZEL, L. N. und J. L. LUSH (1942).

The efficiency of three methods of selection. J. Heredity 33: 393-399. 
JAGANNATHA, S., J. F. KEOWN und L. D. VAN VLECK (1998).

Estimation of relative economic value for herd life of dairy cattle from profile equations. J. Dairy Sci. 81: 1702-1708.

JEROCH, H., W. DROCHNER und O. SIMON (1999).

Ernährung landwirtschaftlicher Nutztiere - Ernährungsphysiologie, Futtermittelkunde, Fütterung. Stuttgart, Eugen Ulmer Verlag.

KALTENECKER, T. (1999).

Ökonomische Entwicklungen in der bayerischen Milchproduktion unter dem Einfluss veränderter agrarpolitischer Rahmenbedingungen. Technische Universität. München, Deutschland. Diss.

Keller, D. S. und F. R. Allaire (1990).

Economic weights for genetic changes in milk component yields at the herd level. J. Dairy Sci. 73: 1631-1643.

Koenen, E., P. BERENTSEN und A. F. Groen (1999).

Economic relevance of selection for live weight and feed intake capacity in dairy cattle. Proceeding of the 1999 Interbull meeting, Zürich, Switzerland, Interbull Bulletin. no. 22. 121-125.

Koenen, E., P. B. M. Berentsen und A. F. Groen (2000).

Economic values of live weight and feed-intake capacity of dairy cattle under Dutch production circumstances. Livest. Prod. Sci. 66: 235-250.

KRogMEIER, D., B. LUNTZ und K.-U. GöTZ (2006).

Untersuchungen zur ökonomischen Bewertung von Exterieurmerkmalen bei Braunvieh und Fleckvieh anhand von Jungkuh-Auktionsdaten. Züchtungskunde 78(6): 464-478.

KRÖMKER, V. (2006).

Risikoanalyse für das Auftreten von Mastitiden. Universität Göttingen. 24.04.2006. Vortrag, Seminar für Nutztierwissenschaften.

KTBL (2005).

Faustzahlen für die Landwirtschaft. Darmstadt, Kuratorium für Technik und Bauwesen in der Landwirtschaft e. V.

KULAK, K., H. M. NiELSEN und E. STRANDBERg (2003).

Comparison of economic values for dairy cattle traits in Nordic countries. Nordic Agriculture in Global Perspectives, Turku, Finland, Nordic Assosiation of Agricultural Scientists $22^{\text {nd }}$ Congress. 20. 
KULAK, K., H. M. NiELSEN und E. STRANDBERG (2004).

Comparison of economic values for dairy cattle traits across the Nordic countries. in: Economic values for production and functional traits in dairy cattle breeding goals derived by stochastic simulation. H. M. NIELSEN: 127-145.

LANDWIRTSCHAFTSKAMMER. LWK Schleswig-Holstein (2004).

Betriebszweigauswertung 2004. Kiel.

LFL. Bayerische Landesanstalt für Landwirtschaft (2004a).

Betriebszweigauswertung 2004. München.

LFL. Sächsiche Landesanstalt für Landwirtschaft (2004b).

Melkbarkeit und Eutergesundheit. Dresden. Schriftenreihe 9. Jahrgang, Heft 9.

LFL. Bayerische Landesanstalt für Landwirtschaft (2005).

Landwirtschaftliche Erzeugerpreise in Bayern - Datensammlung für die Landwirtschaft. München.

LKV. Landeskontrollverband Schleswig-Holstein e. V. (2004).

LKV-Jahresbericht Prüfjahr 2004. Kiel.

LKV. Landeskuratorium der Erzeugerringe für tierische Veredlung in Bayern e.V. (2005).

Leistungs- und Qualitätsprüfung in der Rinderzucht in Bayern 2005. München.

MACK, G. (1996).

Wirtschaftlichkeit des züchterischen Fortschritts in Milchviehherden - Gesamtbetriebliche Analyse mit Hilfe eines simultan-dynamischen Linearen Planungssatzes. Universität Hohenheim. Stuttgart, Deutschland. Diss.

MACK, G., A. WEIDELE, O. DISTL und J. ZEDDIES (1997).

Grenznutzen der Leistungsverbesserung in der Milchproduktion. Züchtungskunde 69(5): 322-333.

MELTON, B. E., E. O. HEADY und R. L. WiLlHAM (1979).

Estimation of economic values for selection indices. Anim. Prod. 28: 279286.

MieSENBERGER, J. (1997).

Zuchtzieldefinition und Indexselektion für die österreichische Rinderzucht. Universität für Bodenkultur. Wien, Österreich. Diss. 
MieSENBERGER, J. (1999)

Der ökonomische Gesamtzuchtwert. ZAR - Zuchtziele beim Rind. 15-26.

MIESENBERGER, J., J. SölKNER und A. EßL (1996).

Der Gesamtindex - Zusammenfassung der Merkmale. Seminar des genetischen Ausschusses der ZAR, Salzburg, Zentrale Arbeitsgemeinschaft österreichischer Rinderzüchter. 40-48.

Miesenberger, J., J. SölKNER und A. EßL (1997).

Economic weights for fertility and reproduction traits relative to other traits and effects of including functional traits into a total merit index. International workshop on EU concerted action - Genetic improvement of functional traits in cattle (GIFT), Grub, Germany, Interbull Bulletin no. 18. 78-84.

Miglior, F., B. L. MUIR und B. J. VAN DoormaAl (2005).

Selection indices in Holstein cattle of various countries. J. Dairy Sci. 88: $1255-1263$.

MoAV, R. und J. MoAV (1966).

Profit in broiler enterprise as a function of egg production of parent stocks and growth rate of their progeny. Br. Poult. Sci. 7: 5-15.

MULDER, H. und G. JANSEN. (2001, July 19, 2001).

Derivation of economic values using lifetime profitability of Canadien holstein cows. www.aps.uoguelph.ca/ gjansen/pubs/01mulder.pdf.

Mundoz-LunA, A., S. B. S. YADAV und L. DempFle (1988).

Derivation of economic weights for several traits for the Rubia-Gallega cattle in Spain. J. Anim. Breed. Genet. 105: 372-383.

NIELSEN, H. M. (2004).

Economic values for production and functional traits in dairy cattle breeding goals derived by stochastic simulation. The Royal Veterinary and Agricultural University. Frederiksberg, Denmark. Ph. D.

OECD/FAO. (2005).

Agricultural Outlook 2005-2014. Paris.

ØSTERGAARD, S., J. T. SøRENSEN und H. HOUE (2003).

A stochastic model simulating milk fever in a dairy herd. Prev. Vet. Med. 58: $125-143$. 
ØSTERgaARD, S., J. T. SøRENSEN und A. R. KRISTENSEN (2000).

A stochastic model simulation the feeding-health-production complex in a dairy herd. J. Dairy Sci. 83: 721-733.

Pärna, E., K. Pärna und I. A. DEWi (2003).

Economic value of milk production and functional traits in the Estonian Holstein Population. EFITA 2003 Conference, Debrecen, Hungary. 352-359.

Pearson, R. E. und R. H. Miller (1981).

Economic definition of total performance breeding goals, and breeding values for dairy cattle. J. Dairy Sci. 64: 857-869.

Pérez-Cabal, M. A. und R. Alenda (2003).

Lifetime profit as an individual trait and prediction of its breeding values in Spain Holstein cows. J. Dairy Sci. 86: 4115-4122.

Pieters, T., F. Canavesi, M. Cassandro, E. Dadati und J. A. M. van ArenDONK (1997).

Consequences of differences in pricing systems between regions on economic values and revenues of a national dairy cattle breeding scheme in italy. Livest. Prod. Sci. 49: 23-32.

REINHARDT, F. (1998).

Beschreibung des Gesamtzuchtwertes (RZG) für Schwarzbunt, Rotbunt, Rotvieh/Angler. Aktueller Stand und neue Entwicklungen der Zuchtwertschätzung beim Rind, Verden, DGfZ-Schriftenreihe. Heft 11. 117-121.

REINHARDT, F. (2006).

Persönliche Mitteilung. 18. Dezember 2006.

REINSCH, N. (1993).

Berechnung wirtschaftlicher Gewichtungsfaktoren für sekundäre Leistungsmerkmale beim Fleckvieh. Technische Universität. München-Weihenstephan, Deutschland. Diss.

REISCH, E. (1962).

Die lineare Programmierung in der landwirtschaftlichen Betriebslehre. München - Basel - Wien, BLV Verlagsgesellschaft.

RENSING, S. (2007).

Persönliche Mitteilung. 7. Mai 2007. Verden. 
ROGERS, G. W. (1994).

Requirements and uses of evaluations for health and reproductive traits. 5th World Congress on Genetics Applied to Livestock Production, Guelph, Canada.

SCHIERENBECK, S. (2006).

Einflussfaktoren auf die Auktionspreise von abgekalbten Färsen im Zuchtgebiet der NORDRIND GmbH. Georg-August-Universität. Göttingen, Deutschland. Masterarbeit.

SCHILD, H. J. und E. NIEBEL (1998).

Zuchtwertschätzung auf Fleischleistung. Aktueller Stand und neue Entwicklungen der Zuchtwertschätzung beim Rind, Verden.

SCHÖNMUTH, G. (1999).

Entwicklungslinien der deutschen Schwarzbunt- und Holsteinzucht im 20. Jahrhundert. Festkolloquium zu Ehren von Georg Schönmuth. 121-169.

SCHÜLER, L., H. H. SWALVE und K.-U. GÖTZ (2001).

Grundlagen der Quantitativen Genetik. Stuttgart, Verlag Eugen Ulmer.

\section{SEUFFERLEIN, H. J. (2005).}

Aktueller Stand der Umbewertung bei der Fett-Eiweißbezahlung. Grub. 5. Oktober 2005. Rasseausschuss Fleckvieh der Arbeitsgemeinschaft Süddeutscher Rinderzuchtverbände und Besamungsorganisationen e. V.

SMITH, C. (1983).

Effects of changes in economic weights on the efficiency of index selection. J. Anim. Sci. 56(5): 1057-1064.

SMITH, C. (1985).

Scope for selecting many breeding stocks of possible economic value in the future. Anim. Prod. 41: 403-412.

\section{SMITH, C., J. W. JAMES und E. W. BRASCAMP (1986).}

On the derivation of economic weights in livestock improvement. Anim. Prod. 43: 545-551.

\section{SöLKNER, J. und W. FUCHS (1987).}

A comparison of different measures of persistency with special respect to variation of test-day milk yields. Livest. Prod. Sci. 16: 305-319. 
SöLKNER, J. und C. FüRST (1998).

Zuchtwertschätzung Zuchtleistung: Nutzungsdauer. Aktueller Stand und neue Entwicklungen der Zuchtwertschätzung beim Rind, Verden. 61-67.

SölKner, J., J. Miesenberger, A. Willam, C. FürST und R. BAUMUng (2000). Total merit indices in dual purpose cattle. Archiv für Tierzucht 43(6): 597608 .

SölKNER, J., A. WILlAM, E. GIERZINGER und C. EgGER-DANNER (1999).

Effects of including conformation in total merit indices of cattle. International Workshop on EU Concerted Action Genetic Improvement of Functional Traits in Cattle (GIFT) - Breeding goals and selection schemes, Wageningen, Netherlands, Interbull Bulletin. no. 23. 143-150.

SøRENSEN, J. T., E. S. KRISTENSEN und I. THYSEN (1992).

A stochastic model simulating the dairy herd on PC. Agricultural Systems 39: 177-200.

SWALVE, H. H. (1998).

Theoretische Grundlagen der Zuchtwertschätzung. Aktueller Stand und neue Entwicklungen der Zuchtwertschätzung beim Rind, Verden, DGfZSchriftenreihe. 11. 7-15.

SWALVE, H. H. und N. GENGLER (1999).

Genetics of lactation persistency. Proc. Int. Symp. Methabolic Stress in Dairy Cows, Occ. Publ. Br. Soc. Anim. Sci. 24. 75-82.

SWALVE, H. H. und K. HöVER (2003).

Untersuchungen an den Ergebnissen der Zuchtwertschätzung von Holstein Bullen und Kühen in Deutschland. Arch. Tierz. 46(2): 113-126.

VAN ARENDONK, J. A. M. (1991).

Use of profit equations to determine relative economic value of dairy cattle herd life and production from field data. J. Dairy Sci. 74: 1101-1107.

VArgas, B., A. F. Groen, M. Herrero und J. A. M. van ArendonK (2002). Economic values for production and functional traits in Holstein cattle of Costa Rica. Livest. Prod. Sci. 75: 101-116.

VEERKAMP, R. F. (1998).

Selection for economic efficiency of dairy cattle using information on live weight and feed intake: A review. J. Dairy Sci. 81: 1109-1119. 
Veerkamp, R. F., P. Dillon, E. Kelly, A. R. Cromie und A. F. Groen (2002).

Dairy cattle breeding objectives combining yield, survival and calving interval for pasture-based systems in Ireland under different milk quota scenarios. Livest. Prod. Sci. 76: 137-151.

VIT (2006)

Beschreibung der Zuchtwertschätzung für Milchleistungsmerkmale, Zellzahl, Exterieurmerkmale, Nutzungsdauer und Zuchtleistungsmerkmale. Vereinigte Informationssysteme Tier i.W.

Von RoHr, P., A. HofER und N. KüNZI (1999).

Economic values for meat quality traits in pigs. J. Anim. Sci. 77: 2633-2640.

WEIDELE, A. (1996).

Die Auswirkungen von Leistungssteigerungen primärer und sekundärer Merkmale beim Rind auf die Wirtschaftlichkeitkoeffizienten und Struktur der Milchviehhaltung in der Bundesrepublik Deutschland - Sektoraler Ansatz. Universität Hohenheim. Stuttgart, Deutschland. Diss.

WEINSCHENCK, G. (1964).

Die optimale Organisation des landwirtschaftlichen Betriebes. Hamburg und Berlin, Paul Parey Verlag.

WEISS, D. ZMP Zentrale Markt- und Preisberichtstelle GmbH (2005). ZMP-Marktbilanz Vieh und Fleisch 2005 - Deutschland - Europäische Union - Weltmarkt. Bonn.

Wolfová, M., J. Pribyl, J. Wolf und R. ZahrádKova (2006).

Effect of subsidy regimes on economic values of functional traits in beef cattle breeding. J. Anim. Breed. Genet. 123: 97-104.

WoOD, P. D. P. (1967).

Algebraic model of the lactation curve in cattle. Nature 216: 164-165.

WÜNSCH, U. (1999).

Berechnung wirtschaftlicher Gewichte für ökonomisch wichtige Leistungsmerkmale anhand sächsischer Milchrindpopulation. Cunersdorf (Sachsen), Sächsische Landesanstalt für Landwirtschaft.

WÜNSCH, U. und U. BERGFELD (2001).

Berechnung wirtschaftlicher Gewichte für ökonomisch wichtige Leistungsmerkmale in der Milchrinderzucht. Züchtungskunde 73(1): 3-11. 
ZuCHTDATA. ZuchtData EDV-Dienstleistungen GmbH (2005a).

Jahresbericht 2005. Wien.

ZUCHTDATA (2005b).

Zuchtwertschätzung beim Rind, Wien, Österreich. 


\section{Danksagung}

Bedanken möchte ich mich bei Prof. Dr. Henner Simianer für die Überlassung des Themas, die hervorragende Betreuung, die uneingeschränkte Unterstützung und das große Vertrauen, das er mir im Laufe der gesamten Arbeit entgegengebracht hat.

PD Dr. Roswitha Baumung möchte ich für die spontane Übernahme des Korreferats danken. Ebenso wie für ihre Einführung in das Amer-Miesenberger-Programm und ihre Hilfestellung in jeglicher Art und Weise.

Dem Deutschen Holstein Verband e.V. (DHV e.V.) und der Arbeitsgemeinschaft Süddeutscher Rinderzüchter e.V. (ASR e.V.) danke ich für die finanzielle Unterstützung des Projektes und die Möglichkeit, meine Vortragsfähigkeit und meine Diskussionstauglichkeit immer wieder neu zu schulen und weiterzuentwickeln.

Dr. Sven König danke ich für die Aufnahme in sein Büro, die gute Zusammenarbeit und die Unterstützung bei allen Fragen bezüglich der Rinderzucht.

Dr. Jörg Dodenhoff (LfL Grub) möchte ich meinen Dank für die gute Zusammenarbeit bei den Rassen Fleckvieh und Braunvieh, die große Diskussionsbereitschaft und die umfassende Unterstützung aussprechen. Dr. Josef Miesenberger (Oberösterreichische Besamungsstation $\mathrm{GmbH}$ ) danke ich für die hervorragende Unterstützung im Umgang mit seinem Programm und die Anteilnahme an meinem Projekt. Dr. Alfred Weidele (RBW) danke ich für die stetige Kritik- und Diskussionsbereitschaft im Laufe des gesamten Projektes.

Bei Friedrich Reinhardt (VIT w. V.) möchte ich mich für die Bereitstellung der Daten und die Überlassung des „RZG-Programms“ bedanken sowie für die lehrreichen Diskussionen.

Bedanken möchte ich mich darüber hinaus bei den Landwirtschaftskammern, Verbänden, Landwirtschaftsämtern etc., die mir Daten zur Bearbeitung meines Projektes zur Verfügung gestellt haben und damit zum Gelingen der Arbeit beigetragen haben.

Besonderer Dank gebührt meinem jetzigen Bürokollegen Sven Schierenbeck. Seine fröhliche, aufgeschlossene, direkte und fürsorgliche Art hat es für mich zu einer Freude gemacht, jeden Tag ins Büro zu kommen. Auch hat er mich bei meinem Projekt ohne Zögern unterstützt, hat jede Ausarbeitung Korrektur gelesen und war immer zur Stelle, wenn Rat und Tat gefragt waren. Darüber hinaus war immer Zeit für Privatgespräche, Austausch von Neuigkeiten und gemeinsame abendliche Unternehmungen. Ich hoffe, dass wir uns auch in Zukunft immer wieder auf der Rinderzuchtbühne begegnen werden. 
Vielen Dank an meine Arbeitsgruppe: Frank, Maria, Dörte, Rike, Ding, Manfred, Tamina, Helge, Frau Döring, Reza - für seinen statistischen und biometrischen Beistand und die Bekanntmachung mit seiner Kultur - und Burchhard Möllers - für die Hilfe bei technischen Problemen jeglicher Art. Besonderer Dank gilt Janet Schmidtko, die sich im Laufe der Zeit zu einer guten Freundin entwickelt hat und immer zur Stelle war, wenn es nötig wurde. Des Weiteren bedanke ich mich bei Stefan Blöttner, Tierzucht Halle, für die hervorragende ,bilaterale“ Zusammenarbeit und die Freundschaft, die sich daraus entwickelt hat.

Nicht zu vergessen sei an dieser Stelle Sabine Rudolphi, die mir als Freundin und Mitbewohnerin während der gesamten Promotionszeit zur Seite stand. Danke, Bine, für deine Unterstützung, deine offene Kritik, deine Hilfe und das immer offene Ohr. Darüber hinaus bin ich froh, durch meine Studienkollegen, die Frühstücker, immer wieder darauf hingewiesen worden zu sein, was praktische Landwirtschaft ist und immer Ablenkung neben der Arbeit erfahren zu haben. Danke auch an Yvonne, Amélie und Volker.

Ich bin froh, auch in den letzten Zügen meiner Arbeit immer Freunde zur Seite stehen zu haben, die zu allen Schandtaten bereit sind, mir den besten Start in den Tag ermöglichen, mit mir frühstücken und Kaffee trinken, mit mir kochen oder mich bekochen, mir die Zeit im Büro überbrücken, mir Unterkunft gewähren, mich zum Lachen bringen und sich sowohl im Zuhören als auch im Kritisieren zu wahren Meistern entwickelt haben. Ich hoffe, eure Freundschaften und eure Lebensfreude werden mich mein Leben lang begleiten.

„Mok wi dat?“ „Mok wi!“” - Danke, Eiko!

Als letztes bleibt mir nur noch den eigentlich größten Dank auszusprechen: Meiner Familie. Neben der immerwährenden Unterstützung, im Beruflichen wie im Privaten, haben sie mir gezeigt, wo mein Zuhause ist. Ich hoffe, ich kann ihnen mit dieser Arbeit einen Teil ihres Vertrauens zurückgeben und ihnen zeigen, dass sie ihre Kinder zu selbstbewussten, eigenständigen Menschen erzogen haben.

„Die schönste Freude erlebt man immer da, wo man sie am wenigsten erwartet hat. “ Antoine de Saint-Exupéry 
Lebenslauf

Name

Geburtsdatum/-ort

Staatsangehörigkeit

Familienstand

Eltern

Berufliche Tätigkeit

seit 2004
Bianca Lind

2. März 1980 in Kassel

deutsch

ledig

Herbert Lind, Landwirtschaftsmeister

Sigrid Lind, geb. Kempe

Wissenschaftlicher Mitarbeiter am Institut für Tierzucht und Haustiergenetik der Georg-August-Universität Göttingen, Arbeitsgruppe Tierzucht (Prof. Dr. H. Simianer); derzeit beschäftigt mit der Anfertigung der Dissertation

\section{Studium}

$1999-2004$

Studium der Agrarwissenschaften an der Georg-AugustUniversität in Göttingen, Schwerpunkt Tierproduktion, Abschluss: Master of Science

Schullaufbahn

\begin{tabular}{ll}
\hline $1990-1999$ & $\begin{array}{l}\text { Engelsburg Gymnasium Kassel } \\
\text { Abschluss: Allgemeines Abitur } \\
\text { Grundschule in Holzhausen/Rhwd. }\end{array}$ \\
Praktika & \\
\hline $2003 / 2004$ & $\begin{array}{l}\text { Praktikum in der landwirtschaftlichen Betriebsberatung, Ros- } \\
\text { dorf }\end{array}$ \\
2001 & Dreimonatiges Ergänzungspraktikum \\
1999 & Dreimonatiges Basispraktikum
\end{tabular}

Göttingen, im Mai 2007 